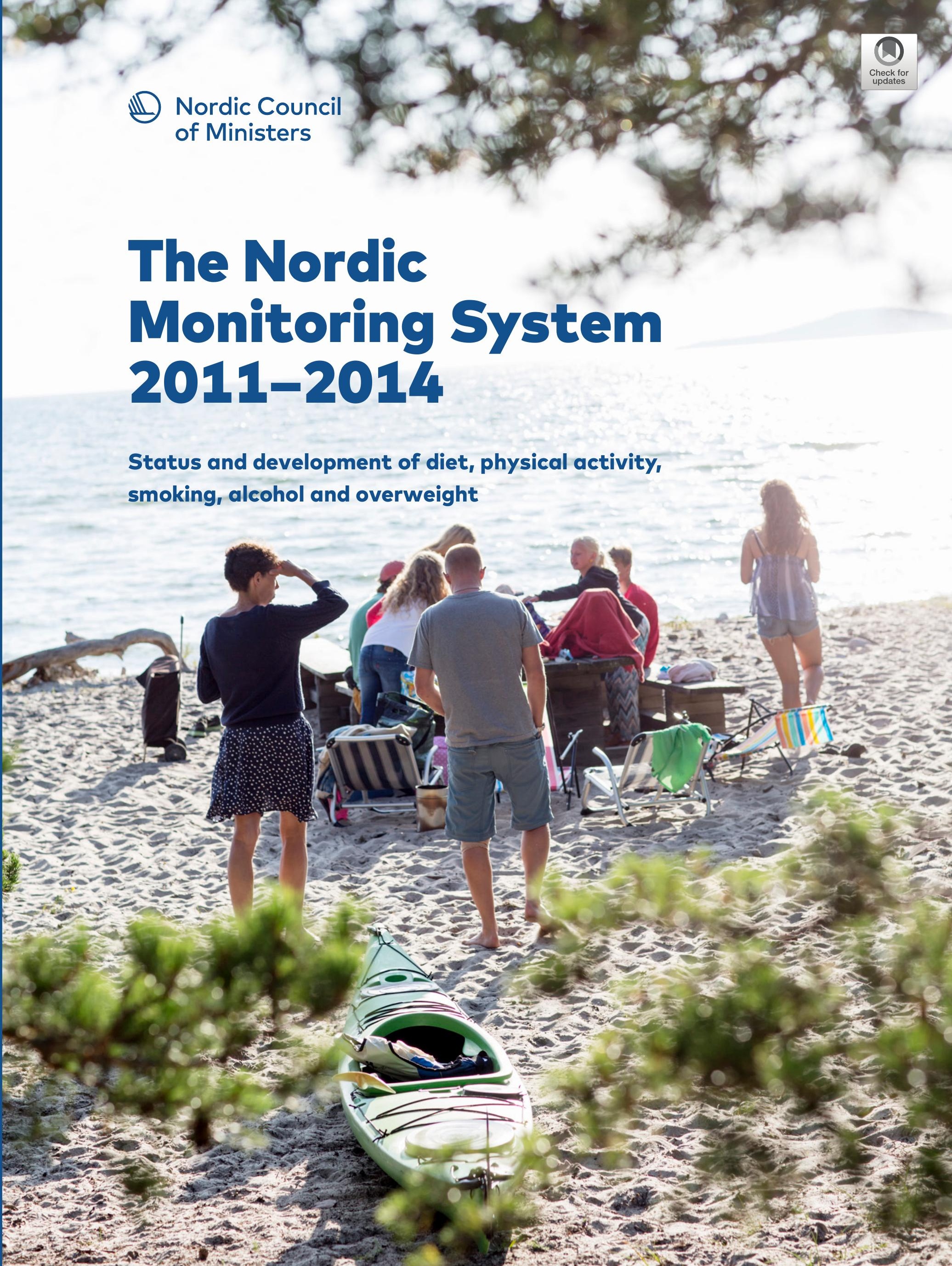





\section{The Nordic Monitoring System}

\section{1-2014}

Status and development of diet, physical activity, smoking, alcohol and overweight

Matthiessen J, Andersen LF, Barbieri HE, Borodulin K, Knudsen VK, Kørup K, Thorgeirsdottir $H$, Trolle E and Fagt $S$

TemaNord 2016:561 
The Nordic Monitoring System 2011-2014

Status and development of diet, physical activity, smoking, alcohol and overweight Matthiessen J, Andersen LF, Barbieri HE, Borodulin K, Knudsen VK, Kørup K, Thorgeirsdottir H, Trolle E and Fagt S

ISBN 978-92-893-4797-6 (PRINT)

ISBN 978-92-893-4798-3 (PDF)

ISBN 978-92-893-4799-O (EPUB)

http://dx.doi.org/10.6027/TN2016-561

TemaNord 2016:561

ISSN $0908-6692$

Standard: PDF/UA-1

ISO 14289-1

(c) Nordic Council of Ministers 2017

Layout: NMR

Cover photo: Johnér

Print: Rosendahls-Schultz Grafisk

Copies 130

Printed in Denmark

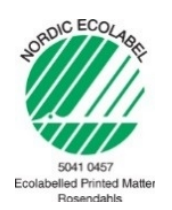

Although the Nordic Council of Ministers funded this publication, the contents do not necessarily reflect its views, policies or recommendations.

\section{Nordic co-operation}

Nordic co-operation is one of the world's most extensive forms of regional collaboration, involving Denmark, Finland, Iceland, Norway, Sweden, the Faroe Islands, Greenland, and Åland.

Nordic co-operation has firm traditions in politics, the economy, and culture. It plays an important role in European and international collaboration, and aims at creating a strong Nordic community in a strong Europe.

Nordic co-operation seeks to safeguard Nordic and regional interests and principles in the global community. Shared Nordic values help the region solidify its position as one of the world's most innovative and competitive. 


\section{Contents}

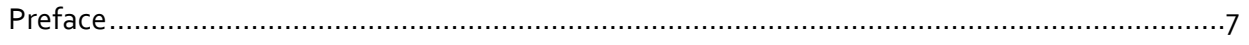

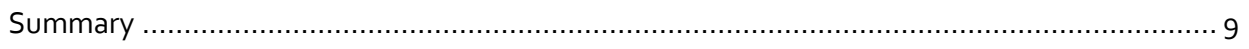

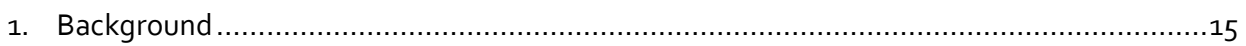

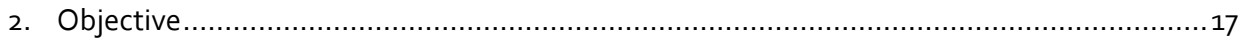

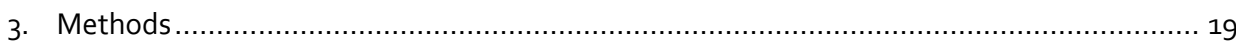

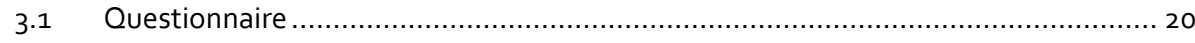

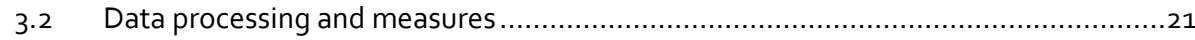

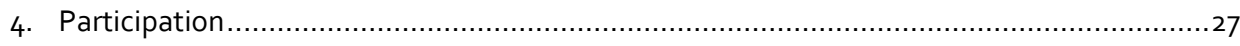

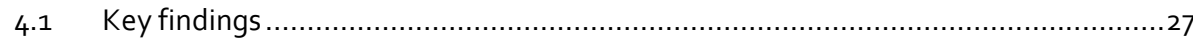

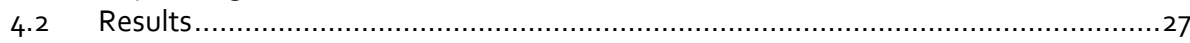

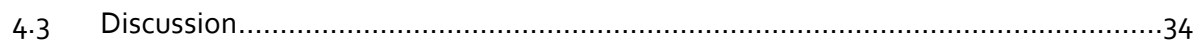

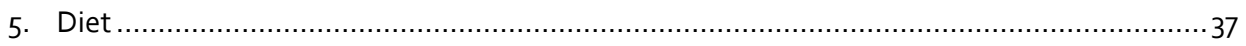

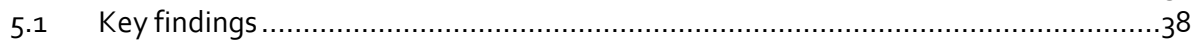

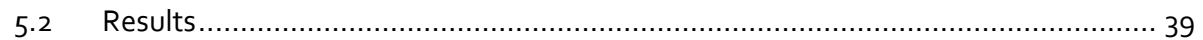

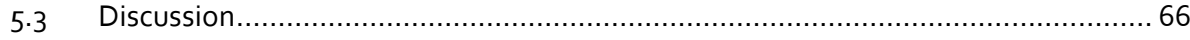

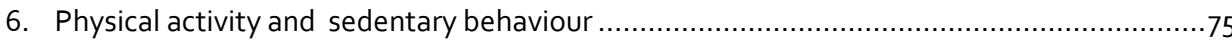

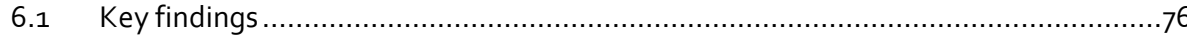

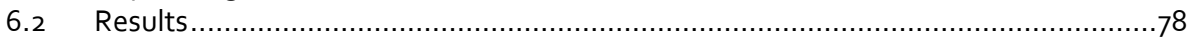

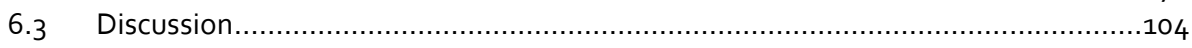

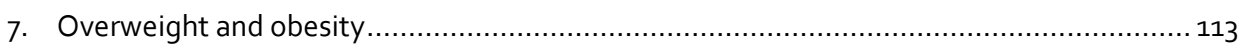

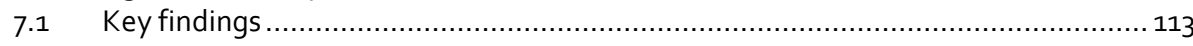

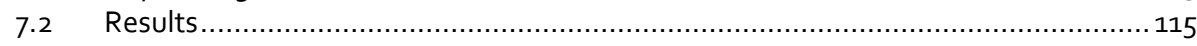

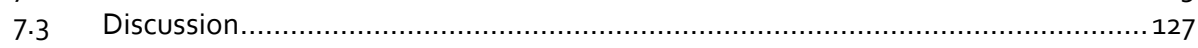

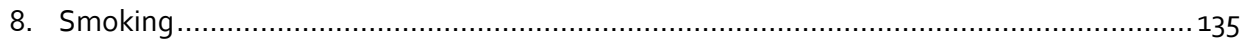

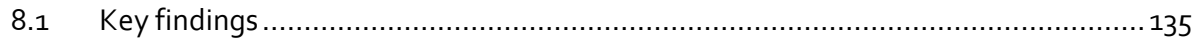

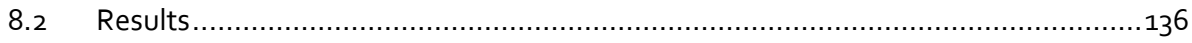

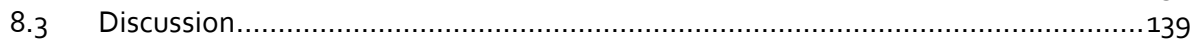

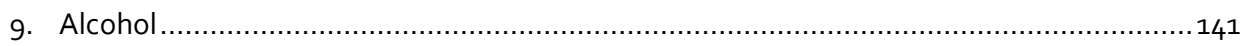

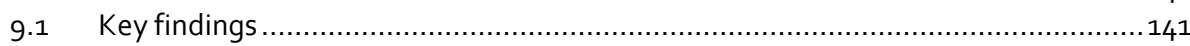

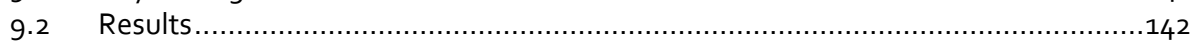

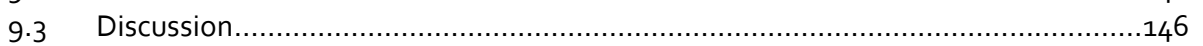




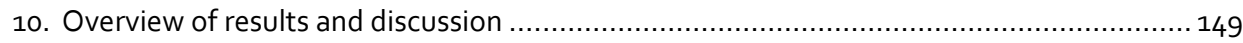

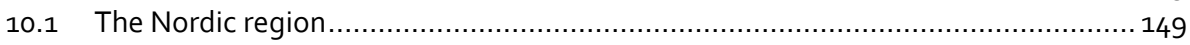

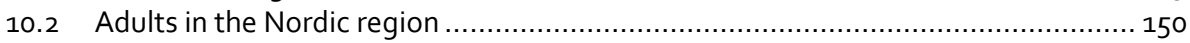

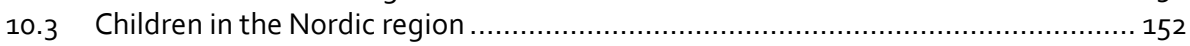

10.4 Natural fluctuations or downward/upward temporal trends ............................. 154

10.5 The most and least favorable health behaviour and weight status in the five Nordic

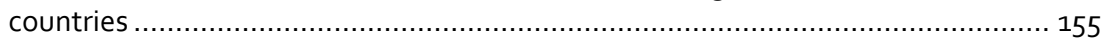

10.6 The development in the five Nordic countries and in the Nordic region .................157

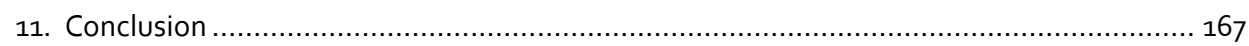

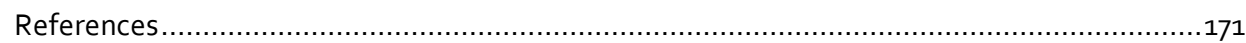

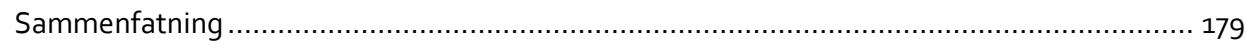

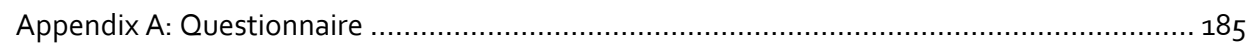

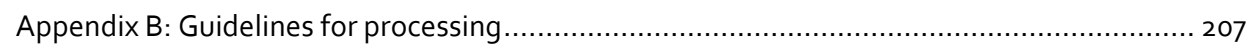

Guidelines for processing data from the Nordic Food Frequency Questionnaire (NFFQ)... 207

Guidelines for processing and categorizing data from the Nordic Physical Activity

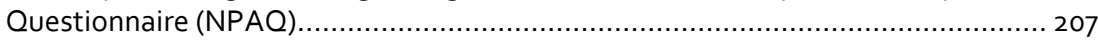

Appendix C: Goals 2011 and Visions 2021 in the Nordic Plan of Action ..............................209

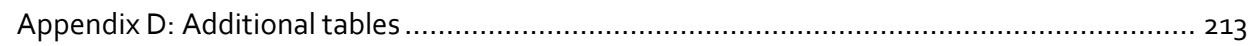




\section{Preface}

The governments in the Nordic countries have through the Nordic Plan of Action on better health committed themselves nationally to address the issue of unhealthy diet, physical inactivity and overweight and enacted policies to promote a healthier lifestyle. The Nordic Council of Ministers has underlined these commitments by formulating common Nordic ambitions in form of Goals 2011 and Visions 2021 on combating unhealthy diet, physical inactivity, and overweight by drawing up the joint Nordic Plan of Action in 2006.

Common goals have been formulated to allow for comparisons, whereby national actions taken in each of the Nordic countries can be assessed. A common ambition is a clear benefit for the Nordic countries when coupled with a common monitoring of effects, an increased sharing of knowledge, a common effort to identify best practice, and increased scientific cooperation. In 2007, a Nordic working group was established with the aim to describe a future Nordic Monitoring System on diet, physical activity and overweight.

In 2008-2010, the monitoring system was developed and validated. In 2011, the first collections of data in the Nordic countries took place and results from this were published in 2012. In 2014, the second data collection was conducted and the current report presents the results from the two data collections.

Valuable contributions have been made by the following: Associate professor Anders Stockmarr, DTU Compute, Denmark, who calculated the population weights and counseled on the statistical analyses. Behavioral scientist Sveinbjörn Kristjánsson, Determinants of Health and Wellbeing, Directorate of Health, Iceland, has contributed to the chapter on smoking.

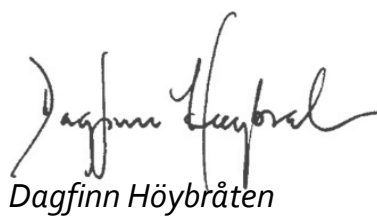

Secretary General

Nordic Council of Ministers 



\title{
Summary
}

\author{
Background and objective
}

Monitoring of diet, physical activity and overweight in the Nordic region is an important element in the Nordic Plan of Action for better health as was put forward by the Nordic Council of Ministers. This report describes the second data collection in 2014 and presents status and the development from 2011 to 2014 as compared to the Goals 2011 and Visions 2021 set by the Nordic Plan of Action.

The objective of the Nordic Monitoring System on diet, physical activity and overweight is to provide status and temporal trend data of diet, physical activity and overweight among adults and children in the Nordic region. Furthermore, objectives are to compare results between the Nordic countries and to compare with regard to recommendations on diet and physical activity, and to examine social inequality in diet, physical activity and overweight. Finally the results are evaluated against the goals and visions in the Nordic Plan of Action to indicate whether diet, physical activity and overweight change favorably or not.

From 2014 the consumption of alcohol as well as smoking behaviour among adults has been included in the data collection, thus enabling comparison of these indicators within the Nordic region.

\section{Method}

Data on diet, physical activity and overweight have been collected in the autumn 2011 and 2014 among 17.775 adults aged 18-65 years and 4.949 children aged 7-12 years in Denmark, Finland, Sweden, Iceland and Norway. A simple random sample of adults and children was drawn from a national register in each of the five Nordic countries. Data were collected by telephone interviews based on a simple questionnaire with validated indicator questions about dietary intake, physical activity and sedentary behaviour. In addition, data on alcohol consumption (adults), smoking behaviour (adults), self-reported body weight and height and a few sociodemografic questions were collected.

The obtained data were checked by the Danish partners at the National Food Institute in Denmark. The further data processing, including cleaning and quality assurance 
and weighting of data according to gender, age and education level in each of the five Nordic countries, were conducted by the Danish partner that also carried out the statistical analyses. The use of weighted data introduces minor changes regarding data from 2011 compared to previous published results.

In 2014, the number of adult respondents in total was 8,622. The pooled participation rate among adults in the Nordic countries was $32 \%$. The number of respondents among children was in total 2,479. The pooled participation rate among children in the Nordic countries was $45 \%$. Among adults the participation rate was lower in 2014 than in 2011 ( $40 \%$ vs. 32\%), while no change was found between survey years among children $(45 \%$ vs. $45 \%)$.

\section{Results}

The results are presented in five chapters on diet, physical activity and sedentary behaviour, overweight and obesity, alcohol, and smoking, respectively. The subsequent chapter includes an overview and discussion of the results.

The emphasis in presenting the results in the report has been put on the key findings in the Nordic region. Results are also presented according to gender, age and education. Status and the development of selected key variables of poor health behaviour and health status are shown in the present report, i.e. unhealthy diet, inactive, high recreational screen time, overweight or obesity $(\mathrm{OW} / \mathrm{OB})$, obesity $(\mathrm{OB})$, smokers (adults in 2014), daily smokers (adults in 2014), mean total alcohol consumption (adults in 2014) and binge drinking last month (adults in 2014). The key variables have been chosen to evaluate Goals 2011 and Visions 2021 in the Nordic Plan of Action on better health and quality of life through diet and physical activity. The key variables were also used to assess and identify the group of individuals in the Nordic region that would most likely achieve the greatest health benefits by getting a healthier lifestyle and weight status. Identifying the groups which would benefit the most from a healthier lifestyle and weight status was done because the governments in the Nordic countries have committed themselves nationally to address the issue of unhealthy diet, physical inactivity and overweight to promote a healthier lifestyle in the Nordic region.

Selected data on country level are presented, in terms of which country has the least/most favorable position with a healthy/unhealthy behaviour and weight status. Finally, the results are evaluated in relation to the Goals 2011 and Visions 2021 in the Nordic Plan of Action. 


\section{Status and development in the Nordic region: 2011-2014}

The main results show that the proportion of adults in the Nordic region with an unhealthy diet was more than $20 \%$ and increased from 2011 to 2014 , while the proportion with a healthy diet decreased and only was $10 \%$ in 2014. The category unhealthy/healthy diet is measured by a dietary quality score including intake of fruits and vegetables, fish, whole grain bread, and foods rich in saturated fat or added sugar. Only with regard to added sugar there was a favorable development among adults in the Nordic region. This favorable development was also seen in Denmark, Finland and Norway while no change was seen in Sweden and Iceland. The other dietary variables showed an unfavorable development among adults, except for the intake of fruits and vegetables that did not change in the Nordic region, but increased in Finland and Sweden. No change in fruit and vegetables was observed in Denmark and Iceland and a decrease was seen in Norway.

One in three adults were physically inactive and three in ten spent more than four hours daily on recreational screen time in the Nordic region. In addition, the prevalence of $\mathrm{OW} / \mathrm{OB}$ and $\mathrm{OB}$ among adults was high. Today, it is almost as common to be overweight or obese as an adult in the Nordic region as being normal weight. Furthermore, the increase in the OB prevalence and in the proportions with an unhealthy diet, of highly inactive and with high recreational screen time among adults is of concern. Even so, an encouring increase in the proportion of highly active was also found. These findings could indicate that an increased polarisation of the physical activity pattern is evident among adults in the Nordic region.

The development of alcohol consumption and smoking behaviour has not been previously reported in the Nordic Monitoring System since these variables were not included until the data collection in 2014. Mean total alcohol consumption was 1.7 times/week and the proportion of binge drinkers was $45 \%$ in 2014 among adults in the Nordic region. The proportion of smokers was $21 \%$ and and the proportion of daily smokers was $14 \%$ in the Nordic region.

Results show that health behaviour and weight status, i.e. diet, sedentary behaviour, smoking, alcohol, overweigt and obesity were less favorable among men than among women in the Nordic region.

Among children in the Nordic region, there were no changes in the overall diet, physical activity and overweight between 2011 and 2014. As for adults there was a favorable development in the Nordic region with regard to added sugar although there was no change in each of the five Nordic countries. Furthermore, the intake of fruits and vegetables and fish increased in the Nordic region. 
Although it is encouraging that the same unfavorable development from 2011 to 2014 is not seen among children as for the adults, the high population levels of unhealthy diet, inactivity and overweight in 2014 are of concern. Even though the proportion among children with an unhealthy diet was $15 \%$, the proportion with a healthy diet was only $10 \%$. The proportion of inactive children is approx. $60 \%$ and therefore high. Also the prevalence of OW/OB and OB was relative high (15\%); and a halt in development has previously been followed by a rise.

Gender differences in health behaviour and weight status are not as clear in children as in adults in the Nordic region. The overall diet and sedentary behaviour were less favorable among boys than among girls, whereas physical activity was less favorable among girls.

\section{Public health challenges in the Nordic countries}

Each of the five Nordic countries is challenged with some specific unfavorable population levels and/or development in health behaviour and/or weight status among adults and/or children compared to the other Nordic countries. Denmark is challenged with unfavorable smoking and alcohol behaviour among adults. Sedentary behaviour may be an increasing public health challenge among adults in Finland taking the most recent development of high recreational screen time into consideration. The population level and the development of the proportion with an unhealthy diet in Sweden are of concern, especially among adults. In addition, the high proportion of inactive children in Sweden is worrying from a public health point perspective even if the level did not change between 2011 and 2014. The current situation and the development of OW/OB and $\mathrm{OB}$ indicate that this is a major public health problem among adults in Iceland. Finally, the high proportion of inactive among adults in Norway may give rise to concern even if no change was detected between 2011 and 2014.

Results show that there is still a need for improvements in all the Nordic countries in some areas. The development among adults has mostly been unfavorable or unchanged in the Nordic countries, while the development has mostly been unchanged or favorable among children. 


\section{Evaluation of Goals 2011 and Visions 2021 for diet, physical activity and overweight}

The evaluation of the main results in relation to Goals 2011 and Visions 2021 in the Nordic Plan of Action reflects the overall development in the Nordic region. The goals aim at a favorable development of diet, physical activity and overweight, while the visions aim at reaching a certain population level in 2021.

The goal of added sugar in the diet, "The intake of added sugar has been reduced", has been fulfilled among adults and children in the Nordic region.

In children, four other goals that have been successfully fulfilled are: "The consumption of fruits and vegetables has increased", "The consumption of fish has increased", "The current trend, where an increasing proportion of children are physically inactive, has been brought to a halt and at best reversed", and "The continuing increase in the proportion of the overweight and obese children has been stopped and at best reversed".

In addition, the goals with regard to social inequality in diet, physical activity and $O W / O B$ also seem to be fulfilled since a decrease or no change in social inequality in diet, physical activity and overweight was found in both adults and children, except for the children regarding diet. However, the way goals on social inequality have been fulfilled was not always beneficial from a public health perspective because health behaviour and weight status has detoriated in the high educated group.

The only vision that is currently fulfilled is the one regarding whole grain bread intake in both adults and children in the Nordic region. However, this vision needs a critical revision since the variable shows quite high values even though the intake of whole grain bread is low and decreasing, and depending on the intake of white bread.

\section{Conclusion}

The Nordic Monitoring System has collected data in 2011 and 2014. In this short three year period, significant changes have occurred in the Nordic region, albeit some are modest in magnitude. Some of the changes in the Nordic Monitoring System may be due to natural variation influenced by different factors such as low participation rate. Thus, based on two data collections, three years apart, it is to early to make too firm interpretations of the development in the Nordic region. The Nordic monitoring has to be repeated before temporal trends may be interpreted more distinctly.

The strength of the Nordic Monitoring System is the high comparability between survey years, age groups, educational levels and countries. Other national surveys cannot 
provide such comparability. However before a third data collection is started, initiatives to improve response rate especially in some of the Nordic countries are needed. Furthermore, there is a need for a critical revision of some of the variables used in the evaluation of the goals and visions in the Nordic Plan of Action.

Some of the visions may be possible to fulfill in 2021 among adults in the Nordic region such as the visions on the intake of added sugar, the target population level of physical activity and social inequality in physical activity and overweight, while other visions such as the intake of fruits and vegetables, fish, and saturated fat, the target population level of OW/OB and $\mathrm{OB}$ and social inequality in diet will be difficult to reach in 2021.

Among children in the Nordic region the only vision that may be possible to fulfill is the target prevalence of $\mathrm{OB}$, while the visions on added sugar, target population level of physical activity in addition to other visions such as the intake of fruits and vegetables, fish, and saturated fat, and the target population level of OW/OB and social inequality in diet will be difficult to reach in 2021.

In conclusion, the big picture of the changes from 2011 to 2014 in diet, physical activity, and overweight shows that the development is heading in the wrong direction among adults in the Nordic region while the development among children is more encouraging, but improvements are still needed. A future third data collection may show if these tendencies will continue or if public health initiatives that target diet, physical activity and overweight among adults and children in the Nordic region will succeed in introducing improvements that lead to healthier behaviour and weight status. Men, 4565 -y-olds and the low educated may be especially relevant groups to target to fulfill the common Nordic ambition of ensuring better health and quality of life on equal terms for all Nordic citizens. 


\section{Background}

Monitoring of diet, physical activity and overweight in the Nordic region is an important element in the Nordic Plan of Action on better health as was put forward by the Nordic Council of Ministers in 2006. The monitoring project was started in 2008 and a joint validated Nordic method for collecting representative data on diet, physical activity and overweight was developed (Fagt et al. 2009 and 2012). In 2011, the first collection of data in all the Nordic countries took place (Rasmussen et al. 2012). This report describes the second data collection in 2014 and presents the development from 2011 to 2014 as compared to the goals and visions set by the Nordic Plan of Action.

The method used for the monitoring is a telephone interview based on a questionnaire containing 15 indicators of diet and six indicators of physical activity and sedentary behaviour as well as self-reported body weight and height and sociodemographic variables of participants. The dietary indicators reflect the nutritional quality of the diet and cover foods included in food based dietary guidelines and related to prevalence of non-communicable diseases. The indicators of physical activity and sedentary behaviour are chosen as these reflect different health aspects related to an active/inactive lifestyle and cover physical activity included in the physical activity recommendations. In addition, BMI calculated from self-reported body weight and height was used as an indicator of body fat to estimate overweight and obesity among adults and children. The indicators are suitable for evaluating the development of diet, physical activity and overweight and whether the goals and visions of the Nordic Plan of Action are met.

In 2014, data on smoking and alcohol intake has also been included in the questionnaire. 



\section{Objective}

The objective of the Nordic monitoring of diet, physical activity and overweight is to provide data on status and changes in the intake of selected foods, physical activity, sedentary behaviour and the prevalence of overweight and obesity in the Nordic countries. Furthermore, the objective is to compare results between countries and to compare intake of certain foods and physical activity with regard to the recommendations on diet and physical activity. A further objective is to examine social inequality in diet, physical activity and overweight. The Nordic Monitoring System of diet, physical activity and overweight makes it possible to examine whether goals and visions in the Nordic Plan of Action on better health are fulfilled and whether diet, physical activity and overweight change in a favorable direction or not in the Nordic region.

Data on diet, physical activity and overweight has been collected in 2011 and 2014 among 17,775 adults (18-65 y) and 4,958 children (7-12 y) in Denmark, Finland, Sweden, Iceland and Norway. From 2014 smoking behaviour as well as the consumption of alcohol among adults has been included in the data collection, thus enabling comparison of these indicators within the Nordic region.

The main emphasis in the present report is on the population levels and the development from 2011 to 2014 of selected key variables (unhealthy diet, inactive, high recreational screen time, overweight and obesity (OW/OB), obesity (OB), smokers (adults in 2014), daily smokers (adults in 2014), mean total alcohol consumption (adults in 2014), binge drinking last month (adults in 2014)). The key variables have been chosen to evaluate goals and visions in the Nordic Plan of Action on better health and quality of life through diet and physical activity. The key findings and the drawn conclusions are based on data of the Nordic region because goals and visions in the Nordic Plan of Action relates to the whole region. In addition, the key variables were also used to assess and identify the group of individuals in the Nordic region and in each of the five Nordic countries that would most likely achieve the greatest health benefits by getting a healthier lifestyle and weight status. Identifying the groups which would benefit the most from a healthier lifestyle and weight status was done because the governments in the Nordic countries have committed themselves nationally to address the issue of unhealthy diet, physical inactivity and overweight to promote a healthier lifestyle in the Nordic region. 



\section{Methods}

Telephone interviews were performed in the five Nordic countries with a validated questionnaire (Fagt et al. 2012). A simple questionnaire with indicator questions on diet, physical activity, smoking, alcohol, body weight and height, and a few sociodemographic questions were used in the Nordic monitoring. It took approximately 15 minutes to perform the telephone interview. The questionnaire contained the same number of questions in all the Nordic countries, except for the questions regarding bread intake which were different in Sweden and Iceland due to other bread habits. Questions on consumption of alcoholic beverages as well as smoking behaviour have been added in the 2014 data collection. Questions on smokeless tobacco (snuff) were not included in Denmark because use of smokeless tobacco has not been very prevalent in Denmark so far.

In each Nordic country a simple random sample of adults and children was drawn from the national register. In Denmark the sample was drawn by Statistics Denmark from the "Central Population Register". In Finland by "Väestörekisterikeskus" (folkeregistercentralen), in Sweden by "Folkbokföringsregistret", in Iceland by "Pjóðskrá" (Registers Iceland) and in Norway the sample was drawn by "Skatteetaten".

A sample of adults aged 18-65 years and children aged 7-12 years in September/October 2014 was drawn in each of the five Nordic contries (Table 1).

Table 1: Sample size

$\begin{array}{lrr}\text { Country } & \text { Adults }(\mathrm{n}) & \text { Children (n) } \\ \text { Denmark } & & 835 \\ \text { Finland } & 3,935 & 1,250 \\ \text { Sweden } & 5,000 & 1,250 \\ \text { Iceland } & 7,000 & 906 \\ \text { Norway } & 3,712 & 906 \\ \end{array}$

The goal was to carry out at least 2,000 interviews on adults and 500 interviews on children (one of the parents were interviewed) in each of the five Nordic countries. Respondents received an information letter one week before the telephone interview. If no contact was obtained, contact was tried up to eight times on different weekdays and times of the day. If no contact was achieved after eight attempts, this was recorded as 
"no contact". Reason not to participate was recorded and gender, age, and postal code of non-responders were registered.

Telephone interviews were carried out in autumn/winther 2014 (September to December) in all Nordic countries. However, the sample was not received at the planned time in all countries. Therefore, the telephone interviews did not start at the same date (Table 2). The first interviews were carried out September $3^{\text {rd }} 2014$ and the last interviews were finished December $5^{\text {th }} 2014$.

Table 2: First and last interviewdate among adults (18-65y) and parents of children (7-12 y). NORMO 2014

\begin{tabular}{lcccc} 
& \multicolumn{2}{c}{ Adults } & \multicolumn{2}{c}{ Children } \\
Country & First interview & Last interview & First interview & Last interview \\
Norway & $5 / 11$ & $5 / 12$ & $5 / 11$ & $5 / 12$ \\
Sverige & $22 / 9$ & $4 / 11$ & $22 / 9$ & $4 / 11$ \\
Iceland & $26 / 9$ & $17 / 11$ & $3 / 11$ & $19 / 11$ \\
Finland & $17 / 10$ & $29 / 11$ & $17 / 10$ & $29 / 11$ \\
Danmark & $3 / 9$ & $16 / 11$ & $20 / 9$ & $12 / 11$ \\
\hline
\end{tabular}

SFI, The Danish National Centre for Social Research coordinated the data collection in all the Nordic countries. In Denmark, data were collected by SFI-Survey. Norstat collected data from Sweden, Finland and Norway, while Maskina collected data in Iceland.

\subsection{Questionnaire}

The English versions of the questionnaires for adults and children are shown in Appen$\operatorname{dix} A$. The questionnaires have been translated into the five Nordic languages and are close to be identical. The food frequency questionnaire used for adults and children included questions on the intake of various types of bread, vegetables, fruits and berries, fish, sausages, candy and chocolate, cakes and biscuits, full fat cheese, fruit juice, sugarsweetened and sugar-free soft drinks and energy drinks. Furthermore, questions regarding type of fat used for spread and cooking were included. Questions on physical activity during leisure-time included questions on time spent on moderate and vigorous physical activity during the last week as well as daily time spent on sedentary recreational screen time (TV and computer) during the last week. In addition, questions on occupational (adults) and leisure-time physical activity level were part of the physical activity questionnaire. Sociodemographic questions on gender, age, education (parental education for children), urbanization and household composition were also included. Finally, questions on body weight and height were part of the questionnaire. In 
2014, the questionnaire also included questions on consumption of alcoholic beverages and smoking behaviour.

\subsection{Data processing and measures}

Data for 2011 and 2014 were handled and processed in the same way. Data were cleaned for coding errors and answers judged unrealistic before analyses. Guidelines for processing and categorizing dietary and physical activity data are shown in appendix B. To account for non-representativeness, data were weighted according to gender, age and education using census data. The use of weighted data introduces a minor change regarding data from 2011 compared to previous published results (Rasmussen et al. 2012).

\subsubsection{Measures}

Selected indicators for diet, physical activity and overweight mentioned in the Nordic Plan of Action on better health (Nordic Council of Ministers, 2006) are presented below. These indicators were chosen to evaluate if Goals 2011 and Visions 2021 in the Nordic Plan of Action have been fulfilled. The ambitions with the plan are to promote a healthier lifestyle in the Nordic countries through diet and physical activity. Appendix C shows Goals 2011 and Visions 2021 and how they are evaluated.

\subsubsection{Sociodemographic data}

Gender, age and education (parental education in children) were used as key background variables. Adults were categorized into three age groups: 18-24-, 25-44- and 45-64-y-olds, while children were analysed as one group (7-12-y-olds). Education was categorized into three groups as well: Low (basic education), medium (vocational education or upper secondary education) and high (short, medium or long higher education). Usually the educational level of the mothers was analysed in children $(87.8 \%$ of all children in 2014).

In addition, data for key variables (unhealthy diet, inactive, high recreational screen time, overweight or obesity (OW/OB), obesity (OB), smokers (adults), daily smokers (adults), mean total alcohol consumption (adults), binge drinking last month (adults)) are also shown for urbanisation and household composition in appendix $D$, but data were not analysed statistically. Urbanisation was categorized into five groups: the Capital and suburb, city > 50,000 inhabitants, city 20,000-49,999 inhabitants, town 1,000- 
19,999 inhabitants and countryside. Household composition was categorized into three groups: Single, adult(s) living with child(ren), adult(s) living with other adult(s).

\subsubsection{Diet}

A dietary index score was calculated and used to report the overall dietary quality. In Table 3 it is shown how to calculate the diet quality scores (o-2 points according to intake frequency of different foods). The diet quality score has values from 0-12 points and scores between 1-4 indicates an unhealthy diet, scores between 5-8 indicates a medium healthy diet whereas scores 9-12 indicate a healthy diet, and only 12 indicate an optimal diet.

Table 3: Categorisation of answers from the diet questionnaire (FFO) among adults and children

\begin{tabular}{lcccc} 
& Onit & $\mathbf{p}$ & $\mathbf{1}$ & $\mathbf{p}$ \\
\hline Fruits and vegetables & Times per day & $<3$ & $3-4$ & $\geq 5$ \\
Bread (wholemeal, rye, hard) & Slices per day & $<1$ & $1-2$ & $\geq 3$ \\
Fish and seafood & Times per week & $<1$ & $1-2$ & $\geq 2$ \\
Candy, cakes, soft drinks, pommes frites & Times per week & $\geq 7$ & $3-6$ & $<3$ \\
Fat on bread & Type & $\geq 60 \%$ fat & $\leq 40 \%$ or no spread & - \\
Cheese & Times per week & $\geq 4$ & $1-3$ & $\leq 1$ \\
Sausages & Times per week & $>1$ & $\leq 1$ & - \\
\hline
\end{tabular}

Intake of fruits and vegetables, fish and whole grain bread were analysed. Moreover, the proportion meeting dietary guidelines for fruit and vegetables, fish and whole grain bread and the proportions with a high consumption of sugar-rich foods, a high consumption of foods rich in saturated fat and with an unhealthy diet (lowest diet quality score) were analysed in adults and children.

The proportion with a high consumption of sugar-rich foods was calculated on basis of the total weekly frequency intake of candy, chocolate, cakes and soft drinks. The proportion with a high consumption of foods rich in saturated fats were calculated on basis of frequency intake of full fat cheese and type of fat used on bread and for cooking. Table 4 shows how the scores are calculated. The sugar index score has values from o-2 points and a score of $\mathrm{o}$ indicates a high frequency consumption of sugar-rich foods, a score of 1 indicates a medium frequency consumption of sugar-rich foods whereas a score of 2 indicates a low frequency consumption of sugar-rich foods.

The saturated fat score has similar values from o-2 points and a score of o indicates a high consumption of foods rich in saturated fats whereas a score of 1 indicates a medium consumption of foods rich in saturated fats and a score of 2 indicates a low consumption. 
The dietary quality score is based on a dietary index developed in Sweden (Becker 2009) and has previously been used when reporting dietary data in NORMO (Rasmussen et al. 2012)

\begin{tabular}{|c|c|c|c|c|}
\hline & Unit & op & $1 p$ & $2 p$ \\
\hline \multicolumn{5}{|l|}{ Sugar index score: } \\
\hline $\begin{array}{l}\text { Total sum of intake of cakes, candy/chocolate and soft drinks } \\
\text { (sugar sweetened) }\end{array}$ & Times per week & $\geq 7$ & $3-6$ & $<3$ \\
\hline \multicolumn{5}{|l|}{ Saturated fat score: } \\
\hline Intake of full fat cheese & Times per week & $\geq 4$ & $1-3$ & $<1$ \\
\hline $\begin{array}{l}\text { Type of fat used on bread: Margarine }(<40 \% \text { fat), margarine ( }< \\
40 \% \text { fat) with added phytosterols, do not use fat on bread }\end{array}$ & Use & No & Yes & \\
\hline $\begin{array}{l}\text { Type of fat used for cooking: Oils, fluid margarine, margarine } \\
\text { (<40\%fat) with added phytosterols, do not use fat for cooking }\end{array}$ & Use & No & Yes & \\
\hline
\end{tabular}

\subsubsection{Physical activity and Sedentary behaviour}

Time spent on moderate and vigorous intensity physical activity (MVPA) and screen time ( $T V+$ +computer time) during leisure-time was assessed in adults and children. Furthermore, vigorous intensity physical activity (VPA) and moderate intensity physical activity (MPA) was calculated in adults. MPA was calculated among adults by subtracting VPA from MVPA.

Classifications of physical activity are shown in Table 5 and have previously been used when reporting physical activity data in NORMO (Fagt et al. 2011, Rasmussen et al. 2012). Physically inactive is insufficiently active and is defined as a failure to meet the physical activity recommendations while the complimentary proportion, i.e. $100 \%$ minus inactive, is defined as physically active and is the proportion who meet the physical activity recommendations. The definition of physical inactivity is in agreement with the definition in the Nordic Nutrition Recommendations 2012 (NNR 2012). High recreational screen time ( $>4$ hours/day) was used as an indicator of sedentary behavior during leisure-time (see Chapter on Physical activity and sedentary behaviour). The proportions of highly inactive, inactive, active, highly active (adults) and with high recreational screen time were analysed in adults and children. For reason of comparison with previously reported NORMO data (Rasmussen et al. 2012) and to evaluate Vision 2021 
on physical activity, the proportions of highly inactive, inactive, active and highly active were analysed in the same way even if physical activity recommendations have changed among adults (NNR 2004).

Table 5: Classifications of physical activity among adults and children

\begin{tabular}{|c|c|}
\hline Activity level & Definitions \\
\hline $\begin{array}{l}\text { Highly inactive (adults, children)- } \\
\text { subgroup of inactive }\end{array}$ & $\begin{array}{l}\text { Not meeting physical activity recommendations (almost no MVPA) - classified as } \\
\text { an inadequate activity level }\end{array}$ \\
\hline Inactive (adults, children) & $\begin{array}{l}\text { Not meeting physical activity recommendations (insufficient MVPA) - classified as } \\
\text { an inadequate activity level }\end{array}$ \\
\hline Active (adults, children) & $\begin{array}{l}\text { Meeting minimum physical activity recommendations (meeting MVPA recommen- } \\
\text { dations (children), MPA or VPA recommendations or meeting the recommendation } \\
\text { by a combination of MPA and VPA (adults)) - classified as an adequate activity } \\
\text { level }\end{array}$ \\
\hline $\begin{array}{l}\text { Highly active (adults) - } \\
\text { subgroup of active }\end{array}$ & $\begin{array}{l}\text { Meeting full physical activity recommendations (meeting MPA and VPA recom- } \\
\text { mendations) - classified as an optimal activity level }\end{array}$ \\
\hline
\end{tabular}

\subsubsection{Smoking and alcohol}

Smoking and alcohol were assessed for the first time in NORMO 2014. Therefore it is only possible to examine the population levels of smoking behaviour and alcohol consumption. The proportions of smokers (daily or occasionally smokers) and daily smokers, mean total alcohol consumption and the proportion of binge drinkers during the last month were analysed among adults. The total alcohol consumption is the sum of intake of beer, wine and spirit calculated as times per week. Proportion of binge drinkers was defined as adults drinking $\geq 5$ units of alcohol at one drinking occasion at least once during the last month.

\subsubsection{Overweight and obesity}

BMI was used as an indicator of body fat to estimate overweight and obesity among adults and children and calculated from self-reported (parent-reported in children) body weight and height. BMI was calculated by dividing body weight in kilograms by the square of height in metres $\left(\mathrm{kg} / \mathrm{m}^{2}\right)$. Adults were classified as overweight or obese if $\mathrm{BMI} \geq 25$ and as obese if $\mathrm{BMI} \geq 30$ (WHO, 2000). Children were classified as overweight or obese and obese according to the International Obesity Task Force age- and genderspecific BMI cut-off points corresponding to BMI values of 25 and $30 \mathrm{~kg} / \mathrm{m}^{2}$, respectively, for adults (Cole et al. 2000). 


\subsubsection{Social inequality in overweight and in health behaviour}

Education (parental education in children) was used as an indicator of socioeconomic position. To estimate the magnitude of the social difference in overweight or obesity and obesity and in health behaviour (diet, physical activity, sedentary behaviour, smoking and alcohol) in the Nordic countries, the relative difference between the highest (high) and the lowest (low) education group was calculated as suggested by Giske et al. (2009). Relative differences were considered small if they were $\leq 10 \%$, moderate if they were between $10 \%$ and $20 \%$ and large if they were $\geq 20 \%$. A negative social difference is caused by a higher value in the low education group than in the high education group and vice versa.

\subsubsection{Statistics}

Data were analysed for adults and children separately and for each gender and Nordic country (Denmark, Finland, Sweden, Iceland, Norway) and for the Nordic region (Denmark + Finland + Sweden + Iceland + Norway).

Means and 95\% confidence intervals $(\mathrm{Cl})$ were presented for continuous data, ${ }^{1}$ while proportions and $95 \% \mathrm{Cl}$ were presented for categorical data. Significance testing was performed using t-tests and Chi-square tests to examine differences between survey years, gender, age groups (adults), education groups, and countries.

No significance testing was performed to examine differences between survey years 2011 and 2014 for the social difference in diet, physical activity, and overweight. A relative difference between survey years below 5 percentage points was considered as no change.

To account for non-representativeness, data were weighted using census data from each of the national statistical bureaus in the five Nordic countries (Statistics Denmark, Statistics Finland, Statistics Sweden, Statistics Iceland, Statistics Norway). This was done to reflect the general population of adults and children for each of the Nordic countries with regard to gender, age and education (parental education for children). To make ed-

\footnotetext{
${ }^{1}$ A slight overlap in the confidence intervals was noticed for some of the significant different categorical estimates. Confidence intervals were based on the standard formulas of 1.96 times the standard deviation of data which is a generally accepted measure of uncertainty, but somewhat different from those derived from the true (binomial) distribution of data. The Chi-square test uses the exact distribution and therefore detects the significance. If exact confidence intervals had been used, no overlap between the confidence intervals had occurred. Slightly overlapping confidence intervals were also noticed for some of the significant different continuous estimates. This was due to differences in variation within the analysed groups, and is in line with standard statistical inference theory.
} 
ucational data comparable between the Nordic countries International Standard Classification of Education (ISCED) was used. ${ }^{2}$ The use of weighted data introduces minor changes regarding data from 2011 compared to previous published results (Rasmussen et al. 2012).

The term "Nordic region" was used when reporting results among adults and children in all the Nordic countries. The Nordic region was analysed using weighted averages according to the population size in the five Nordic countries in 18-65- and 7-12-yolds. Sweden counts around $36 \%$ as the largest Nordic country, Iceland about $1 \%$ as the smallest country while Norway, Finland and Denmark counts around $20 \%$ of the total Nordic population in 2011 and 2014. Population data from Eurostat were obtained to estimate the population size in the five Nordic countries.

All reported data were weighted. Differences presented in the text are statistically significant $(p<0.05)$ unless otherwise stated. Differences are tabulated as tendencies when $p<0.10$. However, these are not commented in the text. Statistical analyses were carried out by use of SAS Enterprise Guide version 6.1, SAS Institute Inc., Cary, NC, USA and WPS Workbench version 3.0.1.0.23496.

${ }^{2}$ International Standard Classification of Education, ISCED 2011. UNESCO Institute for Statistics, Montreal Canada 2012. ISCED 1997classification was used for Iceland. 


\section{Participation}

In this chapter results regarding participation rate in the five Nordic countries are reported.

\subsection{Key findings}

- In 2014, the pooled participation rate among adults was 31.7\% in the Nordic countries, varying from $54.4 \%$ in Iceland to $14.0 \%$ in Norway.

- In 2014, the pooled participation rate among children was $45.2 \%$ in the Nordic countries, varying from $71.7 \%$ in Denmark to $29.1 \%$ in Norway.

- A decrease in the participation rate was found from 2011 to 2014 among adults while it was unchanged among children.

- The participants were more often older, women, and had higher level of education than the general population in 2011 and 2014.

\subsection{Results}

\subsubsection{Adults 2014}

The number of invited adults, the number of participants and the participation rate are shown in Table 6. The pooled participation rate in the Nordic countries was $31.7 \%$. The participation rate differed significantly between the countries with Iceland having the highest participation rate (54.4\%) and Norway the lowest rate (14.0\%). 
Table 6: Number of invited adults, eligible sample, number of participants and participation rate in 2014. NORMO 2014

\begin{tabular}{lrrrr} 
Country & Invited $(\mathrm{n})$ & Eligible sample & Respondents $(\mathrm{n})$ & Participation rate (\%) \\
Denmark & 3,929 & 3,884 & 2,005 & 51.7 \\
Finland & 5,000 & 4,996 & 1,719 & 34.4 \\
Sweden & 7,000 & 6,997 & 1,818 & 26.0 \\
Iceland & 3,710 & 3,696 & 2,012 & 54.4 \\
Norway & 7,626 & 7,618 & 1,068 & 14.0 \\
All & 27,264 & 27,191 & 8,622 & 31.7 \\
\hline
\end{tabular}

There were different reasons for non-participation such as not longer eligible, refusal, not reached, or not eligible phone number (Table 7). Iceland had a low number of persons who refused to participate. Finland, Sweden and Norway had a high number of invited persons who could not be contacted. Norway had as well a high number of invited persons who could not be contacted due to telephone number not working.

Table 7: Sample and reasons for non participation in Adults (n). NORMO 2014

\begin{tabular}{|c|c|c|c|c|c|}
\hline & Denmark & Finland & Sweden & Iceland & Norway \\
\hline Sample drawn & 3,929 & 5,000 & 7,000 & 3,710 & 7,626 \\
\hline Not eligible (moved, cannot be found, moved abroad), dead & 42 & 4 & 3 & 14 & 8 \\
\hline Eligible sample & 3,884 & 4,996 & 6,997 & 3,696 & 7,618 \\
\hline Interview accomplished & 2,008 & 1,719 & 1,818 & 2,012 & 1,068 \\
\hline $\begin{array}{l}\text { of these partly accomplished"\# } \\
\text { Reasons for non participation }\end{array}$ & 1 & 2 & 17 & - & 11 \\
\hline Did not want & 820 & 1,207 & 1,850 & 423 & 2,452 \\
\hline No contact & 720 & 1,776 & 2,763 & 1,037 & 2,684 \\
\hline Telephone number not working & 172 & 53 & 310 & 90 & 974 \\
\hline $\begin{array}{l}\text { Sickness, away in interview period, in hospital, language } \\
\text { problems, other reasons }\end{array}$ & 167 & 241 & 256 & 134 & 440 \\
\hline Total non participation & 1,879 & 3,277 & 5,179 & 1,684 & 6,550 \\
\hline
\end{tabular}

Note: ${ }^{\#}$ All interviews included.

Comparison of participation rates between NORMO 2011 and 2014 shows a decrease in the participation rate in all the Nordic countries, except in Iceland (Table 8). The pooled participation rate in the Nordic countries decreased eight percentage points between 2011 and 2014. 
Table 8: Participation rate among adults (\%). NORMO 2011 and 2014

\begin{tabular}{l|rrr}
\hline Country & Participation rate 2011* $(\%)$ & Participation rate 2014 (\%) & Difference (percentagepoints) \\
\hline Denmark & 62.6 & 51.7 & -10.9 \\
Finland & 40.0 & 34.4 & -5.6 \\
Sweden & 37.7 & 26.0 & -11.7 \\
Iceland & 47.3 & 54.4 & 7.1 \\
Norway & 21.3 & 14.0 & -7.3 \\
All & 40.2 & 31.7 & -8.5 \\
\hline
\end{tabular}

Note: $\quad$ *Participation rate in 2011 is calculated slightly differently than in 2014 , but this did not impact results significantly.

\subsubsection{Children 2014}

The number of invited children, the number of participants and the participation rate are shown in Table 9. The pooled participation rate in the Nordic countries was $45.2 \%$ in 2014. The participation rate among children was higher than among adults in the Nordic countries and was highest in Denmark (71.7\%) and lowest in Norway (29.1\%).

Table 9: Number of invited children, eligible sample, number of participants and participation rate. NORMO 2014

\begin{tabular}{lrrrr} 
Country & Invited $(\mathbf{n})$ & Eligible sample & Respondents $(\mathbf{n})$ & Participation rate (\%) \\
Denmark & 835 & 834 & 598 & 71.7 \\
Finland & 1,250 & 1,250 & 500 & 40.0 \\
Sweden & 1,250 & 1,250 & 500 & 40.0 \\
Iceland & 905 & 905 & 514 & 56.8 \\
Norway & 1,250 & 1,205 & 358 & 28.7 \\
All & 5,490 & 5,488 & 2,479 & 45.2 \\
\hline
\end{tabular}

Norway and Sweden had many who refused to participate (Table 10). Finland, Iceland, Sweden and Norway had a large number who could not be contacted. Norway had as well a high number of invited persons who could not be contacted due to telephone number not working. 
Table 10: Sample and reasons for non participation: Children (n). NORMO 2014

Denmark Finland Sweden Iceland Norway

Sample drawn

Not eligible (moved, cannot be found, moved abroad), dead

$\begin{array}{rrrrr}835 & 1,250 & 1,250 & 905 & 1,250 \\ 1 & 0 & 0 & 0 & 1 \\ 834 & 1,250 & 1,250 & 905 & 1,249 \\ 598 & 500 & 503 & 514 & 364 \\ 1 & - & 3 & - & 6 \\ 89 & 158 & 206 & 35 & 416 \\ 108 & 536 & 460 & 328 & 279 \\ 16 & 7 & 51 & 3 & 113 \\ 23 & 49 & 30 & 25 & 77 \\ 236 & 750 & 747 & 391 & 885\end{array}$

Eligible sample

Interview accomplished

of these partly accomplished ${ }^{\#}$

Reasons for non participation

Did not want

No contact

Telephone number not working

Sickness, away in interview period, in hospital, language

problems, other reasons

Total non participation

(entincluded, thus" n" is 500 and 358, respectively.

Note: "Partly interviews from Sweden and Norway are not included, thus " $\mathrm{n}$ " is 500 and 358 , respectively.

Comparison of participation rates between 2011 and 2014 shows a large decrease in the participation rate in Denmark, but no change or an increase in the other Nordic countries. The pooled participation rate in the Nordic countries was similar in 2011 and 2014 (Table 11).

Table 11: Participation rate among children (\%). NORMO 2011 and 2014

\begin{tabular}{lrrr} 
Country & Participation rate 2011*(\%) & Participation rate 2014 (\%) & Difference (percentage points) \\
\hline Denmark & 85.8 & 71.7 & -14.1 \\
Finland & 40.0 & 40.0 & 0 \\
Sweden & 39.9 & 40.0 & 0.1 \\
Iceland & 51.8 & 56.8 & 5.0 \\
Norway & 28.2 & 28.7 & 0.5 \\
All & 45.4 & 45.2 & -0.2 \\
\hline
\end{tabular}

Note: "Participation rate in 2011 is calculated slightly different than in 2014, but this did not impact results significantly.

\subsubsection{Characteristics of the participants}

\section{Adults}

Characteristics of the adult participants in 2011 and 2014 are shown in Table 12. In all the Nordic countries and in both survey years more women than men participated, and participation was highest in the oldest age group ( $45-64$ years). When looking at all the Nordic countries together in 2014 , the highest participation rate was observed among the high educated, while in 2011 the highest participation rate was found among the medium educated. This tendency was also found for Finland, Sweden and Iceland when 
looking at each of the Nordic countries. In Denmark and Norway, the highest participation rate was found among the high educated in 2011 and 2014.

Children

Characteristics of the participating children and the interviewed persons in 2011 and 2014 are shown in Table 13. In all the Nordic countries, almost the same proportion of boys and girls participated. In Finland and Norway, more girls than boys participated in 2011 and 2014 while in Sweden it was the opposite. In Iceland and Denmark, there was a change in the gender having the highest participation rate between 2011 and 2014. It was mainly the mother of the child who was interviewed both in 2011 and 2014, except in Norway in 2011 where a high proportion of fathers were interviewed. When looking at all the Nordic countries, the highest participation rate was seen among children with a high educated parent in 2011 and 2014. This was also found when looking at each of the five Nordic countries, except in Finland. 
Table 12: Characteristics of the adult study population. NORMO 2011 and 2014

\begin{tabular}{|c|c|c|c|c|c|c|c|c|c|c|c|c|}
\hline & \multicolumn{2}{|c|}{ Denmark } & \multicolumn{2}{|c|}{ Finland } & \multicolumn{2}{|c|}{ Sweden } & \multicolumn{2}{|c|}{ Iceland } & \multicolumn{2}{|c|}{ Norway } & \multicolumn{2}{|c|}{ All Nordic countries } \\
\hline & $2011(n, \%)$ & $2014(n, \%)$ & $2011(n, \%)$ & $2014(n, \%)$ & $2011(n, \%)$ & $2014(n, \%)$ & $2011(n, \%)$ & $2014(n, \%)$ & $2011(n, \%)$ & $2014(n, \%)$ & $2011(n, \%)$ & $2014(n, \%)$ \\
\hline \multicolumn{13}{|l|}{ Gender\# } \\
\hline \multirow[t]{2}{*}{ Male } & 1,014 & 976 & 922 & 756 & 885 & 827 & 938 & 895 & 514 & 484 & 4,273 & 3,938 \\
\hline & $46.4 \%$ & $48.6 \%$ & $46.0 \%$ & $44.0 \%$ & $46.9 \%$ & $45.9 \%$ & $46.6 \%$ & $44.5 \%$ & $48.3 \%$ & $45.8 \%$ & $46.7 \%$ & $45.8 \%$ \\
\hline \multirow[t]{2}{*}{ Female } & 1,173 & 1,031 & 1,082 & 961 & 1,001 & 974 & 1,078 & 1,117 & 551 & 573 & 4,880 & 4,656 \\
\hline & $53.6 \%$ & $51.4 \%$ & $54.0 \%$ & $56.0 \%$ & $53.1 \%$ & $54.1 \%$ & $53.4 \%$ & $55.5 \%$ & $51.7 \%$ & $54.2 \%$ & $53 \cdot 3 \%$ & $54.2 \%$ \\
\hline \multicolumn{13}{|l|}{ Age } \\
\hline \multirow[t]{2}{*}{$18-24 y$} & 227 & 230 & 237 & 157 & 249 & 184 & 212 & 164 & 109 & 114 & 1,034 & 849 \\
\hline & $10.4 \%$ & $11.5 \%$ & $11.8 \%$ & $9.1 \%$ & $13.2 \%$ & $10.2 \%$ & $10.5 \%$ & $8.2 \%$ & $10.2 \%$ & $10.8 \%$ & $11.3 \%$ & $9.9 \%$ \\
\hline \multirow[t]{2}{*}{$25-44 y$} & $75^{2}$ & 651 & 649 & 532 & 719 & 699 & 835 & 808 & 445 & 392 & 3,400 & 3,082 \\
\hline & $34.4 \%$ & $32.4 \%$ & $32.4 \%$ & $31.0 \%$ & $38.1 \%$ & $38.8 \%$ & $41.5 \%$ & $40.2 \%$ & $41.8 \%$ & $37.1 \%$ & $37.1 \%$ & $35.9 \%$ \\
\hline \multirow[t]{2}{*}{$45-65 y$} & 1,208 & 1,127 & 1,118 & 1,028 & 918 & 918 & 964 & 1,040 & 511 & 551 & 4,719 & 4,664 \\
\hline & $55.2 \%$ & $56.1 \%$ & $55.8 \%$ & $59.9 \%$ & $48.7 \%$ & $51.0 \%$ & $47.9 \%$ & $51.7 \%$ & $48.0 \%$ & $52.1 \%$ & $51.6 \%$ & $54.3 \%$ \\
\hline \multicolumn{13}{|l|}{ Education ${ }^{\circledR}$} \\
\hline \multirow[t]{2}{*}{ Low (up to 10 y) } & 355 & 215 & 303 & 218 & 217 & 128 & 513 & 447 & 69 & 57 & 1,457 & 1,065 \\
\hline & $16.2 \%$ & $10.7 \%$ & $15.2 \%$ & $12.7 \%$ & $11.7 \%$ & $7.1 \%$ & $24.4 \%$ & $22.3 \%$ & $6.5 \%$ & $5.4 \%$ & $16.0 \%$ & $12.4 \%$ \\
\hline \multirow[t]{2}{*}{ Medium (10-12 y) } & 888 & 689 & 1,026 & 681 & 864 & 752 & 820 & 671 & 383 & 350 & 3,981 & 3,143 \\
\hline & $40.6 \%$ & $34 \cdot 3 \%$ & $51.5 \%$ & $39.8 \%$ & $46.3 \%$ & $41.9 \%$ & $40.9 \%$ & $33.4 \%$ & $36.1 \%$ & $33.2 \%$ & $43.7 \%$ & $36.6 \%$ \\
\hline \multirow[t]{2}{*}{$\operatorname{High}(13+y)$} & 943 & 1,104 & 663 & 814 & 784 & 916 & 674 & 890 & 609 & 649 & 3,673 & 4,370 \\
\hline & $43.1 \%$ & $55.0 \%$ & $33.3 \%$ & $47.5 \%$ & $42.0 \%$ & $51.0 \%$ & $33.6 \%$ & $44.3 \%$ & $57.4 \%$ & $61.3 \%$ & $40.3 \%$ & $50.9 \%$ \\
\hline
\end{tabular}

Note:

\#One participant had missing data on gender.

@Characteristics of the child study population are missing on some respondents. 
Table 13: Gender of the participating child and parental education. NORMO 2011 and 2014

\begin{tabular}{|c|c|c|c|c|c|c|c|c|c|c|c|c|}
\hline & \multicolumn{2}{|c|}{ Denmark } & \multicolumn{2}{|c|}{ Finland } & \multicolumn{2}{|c|}{ Sweden } & \multicolumn{2}{|c|}{ Iceland } & \multicolumn{2}{|c|}{ Norway } & \multicolumn{2}{|c|}{ All Nordic countries } \\
\hline & $2011(n, \%)$ & $2014(n, \%)$ & $2011(n, \%)$ & $2014(n, \%)$ & $2011(n, \%)$ & $2014(n, \%)$ & $2011(n, \%)$ & $2014(n, \%)$ & $2011(n, \%)$ & $2014(n, \%)$ & $2011(n, \%)$ & $2014(n, \%)$ \\
\hline \multicolumn{13}{|l|}{ Gender } \\
\hline Boys & $52.4 \%$ & $49.3 \%$ & $49.2 \%$ & $48.6 \%$ & $54.5 \%$ & $51.6 \%$ & $47.7 \%$ & $50.2 \%$ & $47.9 \%$ & $48.3 \%$ & $50.5 \%$ & $49.7 \%$ \\
\hline \multirow[t]{2}{*}{ Girls } & 290 & 303 & 254 & 257 & 227 & 242 & 271 & 256 & 184 & 185 & 1,226 & 1,243 \\
\hline & $47.6 \%$ & $50.7 \%$ & $50.8 \%$ & $51.4 \%$ & $45 \cdot 5 \%$ & $48.4 \%$ & $52.3 \%$ & $49.8 \%$ & $52.1 \%$ & $51.7 \%$ & $49.5 \%$ & $50.3 \%$ \\
\hline \multicolumn{13}{|c|}{ Gender of the interviewed } \\
\hline Women & $85.1 \%$ & $83.3 \%$ & $91.4 \%$ & $93.8 \%$ & $92.2 \%$ & $88.6 \%$ & $86.4 \%$ & $85.6 \%$ & $57.5 \%$ & $88.8 \%$ & $--^{\epsilon}$ & $87.8 \%$ \\
\hline \multicolumn{13}{|l|}{ Parental education@ } \\
\hline \multirow[t]{2}{*}{ Low (up to 10 y) } & 39 & 47 & 17 & 10 & 8 & 18 & 100 & 70 & 16 & 3 & 180 & 148 \\
\hline & $6.4 \%$ & $7.9 \%$ & $3.4 \%$ & $2.0 \%$ & $1.6 \%$ & $3.6 \%$ & $19.3 \%$ & $13.6 \%$ & $4.6 \%$ & $0.8 \%$ & $7.3 \%$ & $6.0 \%$ \\
\hline \multirow[t]{2}{*}{ Medium (10-12 y) } & 227 & 113 & 245 & 167 & 216 & 147 & 141 & 130 & 119 & 77 & 948 & 634 \\
\hline & $37.3 \%$ & $19.1 \%$ & $49.1 \%$ & $33.4 \%$ & $43.5 \%$ & $29.6 \%$ & $27.3 \%$ & $25 \cdot 3 \%$ & $34.2 \%$ & $21.6 \%$ & $38.4 \%$ & $25.8 \%$ \\
\hline \multirow[t]{2}{*}{$\operatorname{High}(13+y)$} & 343 & 432 & 237 & 323 & 273 & $\begin{array}{r}332 \\
66.8 \%\end{array}$ & $\begin{array}{r}276 \\
52,4 \%\end{array}$ & $\begin{array}{r}313 \\
61.0 \%\end{array}$ & $\begin{array}{r}213 \\
61.2 \%\end{array}$ & $\begin{array}{r}276 \\
776 \%\end{array}$ & $\begin{array}{r}1,342 \\
54,2 \%\end{array}$ & $\begin{array}{r}1,676 \\
68.2 \%\end{array}$ \\
\hline & $56.3 \%$ & $73.0 \%$ & $47.5 \%$ & $64.6 \%$ & $54.9 \%$ & $66.8 \%$ & $53.4 \%$ & $61.0 \%$ & & $77.6 \%$ & & $68.2 \%$ \\
\hline
\end{tabular}

Note: @Data on educational level are missing on some respondents.

£ Not calculated on regional level in 2011. 


\subsection{Discussion}

Declining participation rates in population-based studies are a challenge that has been faced in many western countries during the past decades (Atrostic et al. 2001, de Heer 1999, de Heer and Israels, 1992). Participation in health examination studies, for example, has declined since the 1980 's from around $80 \%$ to as low as $40 \%$ in some recent studies (Grotvedt et al. 2008, HIS/HES Database 2014). In Norway, participation rate in national dietary surveillance studies has declined with about 30 percentage points in both adult and adolescent populations from 1990's to 2015. In the Nordic countries, national postal or telephone surveys have similarly experienced declines from one percentage point to six percentage points over certain time frames, but increased rates have also been experienced in Iceland as is the case with NORMO. The participation rates in the most recent national surveys have varied between $48-69 \%$, suggesting that national surveys have reached better representativeness than NORMO. Of the Nordic countries, only Denmark reached NORMO participation rates of the same magnitude as in the national surveys.

Low participation rates jeopardize the representativeness of results for the general population and bias the interpretations of results. In a Finnish study, it was estimated that the true prevalence of smoking could be higher than the study results suggested because of the decrease in the participation rate (Kopra et al. 2015).

Some previous studies suggest that non-participation is selective. The nonparticipants tend to be more often men (Korkeila et al. 2001, Lundberg et al. 2005, Sogaard et al. 2004, Eaker et al. 1998, Tolonen et al. 2006), from younger age groups (Lundberg et al. 2005, Sogaard et al. 2004, Eaker et al. 1998, Tolonen et al. 2006, Jackson et al. 1996, Shahar et al. 1996), single/not married (Korkeila et al. 2001, Lundberg et al. 2005, Sogaard et al. 2004, Tolonen et al. 2006, Shahar et al. 1996), from lower socio-economic groups (Korkeila et al. 2001, Lundberg et al. 2005, Sogaard et al. 2004, Tolonen et al. 2006, Jackson et al. 1996, Shahar et al. 1996, Cohen and Duffy, 2002, Demarest et al. 2013, Drivsholm et al. 2006), with lower income (Jackson et al. 1996), and living in metropolitan area (Eaker et al. 1998). Several of these characteristics are also known to be associated with health indicators such as daily smoking, alcohol use, and obesity (Borodulin et al. 2012). Furthermore, other characteristics that are previously reported for non-participants include worse self-rated health (Jackson et al. 1996, Cohen and Duffy, 2002, Drivsholm et al. 2006), higher disability benefits (Korkeila et al. 2001, Sogaard et al. 2004, Knudsen et al. 2010), higher hospitalization rates (Drivsholm et al. 2006, Kjoller and Thoning 2005) and more diagnosed diseases than 
survey participants (Jackson et al. 1996). Non-participants have higher all-cause and cause specific mortality in the follow-up studies than survey participants (Hara et al. 2002, Jousilahti et al. 2005, Larsen et al. 2012, Une et al. 2000).

In the Nordic monitoring 2011 and 2014, the participants were older than the general population, and women were overrepresented. Furthermore, more participants with a low or medium education than with a high education refused to participate and this challenge seems to have increased from 2011 to 2014.

Because of this skewed distribution for participation with regard to gender, age, and education compared with the general population in the five Nordic countries, it was decided to weight the data in the statistical analyses.

\subsubsection{How to overcome low participation rate?}

Potential ways to increase participation rates have been reported in earlier studies. Most of the existing literature has reported experiences from health examination studies while postal surveys and interviews studies have received less attention (Eaker et al. 1998; Edwards et al. 2002, Edwards et al. 2009, Tolonen et al. 2014). Some studies have suggested that for example monetary incentives (Edwards et al. 2014, Doody et al. 2003, Fox et al. 1988, Kopf et al. 2000, Schweitzer and Asch, 1995, Singer et al. 1999), shorter questionnaires (Edwards et al. 2002, Kalantar and Talley, 1999), reminders (Fox et al. 1988), pre-notification (Fox et al. 1988, Chun and Robertson, 1995), and first class postage and stamp (Fox et al. 1988, Schweitzer and Asch, 1995) increased participation. Providing both monetary and non-monetary incentives is reported to affect participation rates even better if the incentive is received immediately and not just after having participated (Edwards et al. 2010). Another potential way of increasing participation is related to contacting the subject and potentially creating the invitation more personal. This could be achieved with pre-notifications before sending the questionnaires and reminding about participation (Fox et al. 1988). One Swedish study reported that when questionnaires arrived on Friday, the participation rates were lower as compared to Tuesday (Eaker et al. 1998). One Finnish study sent short messages services (SMS) to mobile phones to remind the subjects about their participation and those receiving the message showed up to health examination more often (Virtanen et al. 2007, Tolonen et al. 2014). In Denmark, the participation rate has increased slightly in the Danish National Survey on Diet and Physical Activity between 2000 and 2011 (Lyhne et al. 2005, Pedersen et al. 2015), probably due to interviewers visiting participant twice and collecting the dietary records from participants. 
In studies that reach the respondents by telephone, the number of attempted contact may be of importance such as 10 attempts resulting in markedly higher participation rates than lesser attempts (Fuchs et al. 2013). Also, the best times to call are in the evenings and Sunday (Weeks et al. 1987), yet these studies are based on older results and more modern techniques should be incorporated such as SMS messages and Emails. Even using the means of social media could be used more efficiently, or at least tested and considered as an option to catch the respondent's attention.

In summary, to achieve high participation rates there is not just one solution. It is likely that best results are achieved when the study invests money in the recruitment phase and tailors different recruitment methods for different sub-populations. The more personalised the invitation and the more rewarding the study sounds, the higher are the participation rates. High participation rates will require higher total costs that are caused by more time in planning the recruitment techniques, more time spent in reaching the respondents, different types of questionnaires for sub-populations, and potential incentives. 


\section{Diet}

In this chapter results on the population levels and the development in dietary intake in the Nordic countries are reported.

Dietary patterns rich in vegetables, pulses, fruits and berries, nuts and seeds, whole grains, fish and seafood, vegetable oils and vegetable oil-based fat spreads and low-fat dairy products are associated with lower risk of non-communicable diseases such as cardiovascular diseases, obesity, type 2 diabetes and some type of cancers (Nordic Council of Ministers 2014).

The dietary assessment method in the Nordic Monitoring System includes 15 foods/food groups, which are considered indicators for the overall dietary quality. In this chapter, the population levels and the development of the consumption of fruits and vegetables, whole grain, fish as well foods rich in saturated fat and added sugar are reported. These variables are mentioned in the "Nordic plan of Action for better health through diet and physical activity", where goals and visions for dietary quality have been set (Nordic Council of Ministers 2006).

A dietary quality score is used to report the overall nutritional quality of the diet. A low dietary quality score between o to 4 is used as an indicator of a diet which is unhealthy in several parameters, e.g. low in fruits and vegetables, whole grain, fish and/or high in foods rich in saturated fat and added sugar. The proportion with a low dietary quality score (unhealthy diet) has been chosen as key variable in this chapter.

The emphasis in this chapter is to report the proportions with an unhealthy diet and fulfilment of selected food based dietary guidelines to cover the important factors regarding dietary quality. Results on unhealthy diet and fulfilling selected foodbased guidelines will only be commented to some extent as these variables are covered by data on the intake of fruits, vegetables, fish and whole grain. Data on the population levels and the development of the proportions with an unhealthy diet and fulfilling selected food based dietary guidelines according to age and education in each of the five Nordic countries are commented in this chapter, but data are not tabulated and only significant findings of the development from 2011 to 2014 will be shown in the text.

When comparing dietary intake between the Nordic countries, only countries where it is possible to identify one or more countries with the highest and/or lowest proportion with an unhealthy diet will be commented in the key findings below. 


\subsection{Key findings}

\subsubsection{Adults}

- In 2014, one in five adults had an unhealthy diet in the Nordic region. The proportion with an unhealthy diet was higher in men than in women $24.7 \% \mathrm{vs}$. 18.4\%). The lowest proportion with an unhealthy diet was observed in Norway and Finland and the highest in Sweden.

- Between 2011 and 2014, the intake of fish and whole grain bread and the proportion with a high consumption of sugar-rich foods decreased while the proportion with a high consumption of foods rich in saturated fat increased.

- In the Nordic region, the proportion with an unhealthy diet increased from $18.2 \%$ to $21.5 \%$ between 2011 and 2014. An increase in the proportion with an unhealthy diet was found in both men and women, 25-44- and 45-65-y-olds and among the the low and high educated. However, the proportion with an unhealthy diet decreased in 18-24-y-olds.

- The social difference in diet (unhealthy diet) was large and decreased slightly between 2011 and 2014.

\subsubsection{Children}

- In 2014, one in seven children had an unhealthy diet in the Nordic region. The proportion with an unhealthy diet was higher in boys than in girls ( $17.5 \% \mathrm{vs}$. 13.7\%). The highest proportion with an unhealthy diet was observed in Sweden.

- Between 2011 and 2014, the intake of fruits and vegetables and fish increased slightly while the intake of whole grain bread and the proportion with a high consumption of sugar-rich foods decreased.

- In the Nordic region, the proportion with an unhealthy diet did not change between 2011 and 2014. No change was also found when gender was analysed. However, the proportion with an unhealthy diet increased in children with a low educated parent and decreased in children with a high educated parent between 2011 and 2014.

- The social difference in diet (unhealthy diet) was large in 2014 and increased between 2011 and 2014 . 


\subsubsection{Conclusions}

The overall diet has become less healthy from 2011 to 2014 among adults in the Nordic region. An unfavorable development was seen for the intake of fish, whole grain and foods rich in saturated fat. Only the intake of added sugar developed favourably. Among children, the overall diet did not change in the Nordic region. Still, some minor improvements of the diet have occurred e.g. increased intake of fruits and vegetables and fish as well as a decreased intake of added sugar. But intake of whole grain decreased.

The social difference in diet was large among adults and children in the Nordic region. Data also indicate that social inequality in diet has not deepened further among adults, but deepended considerably among children.

\subsection{Results}

\subsubsection{Adults}

In 2014, the proportion with an unhealthy diet was $21.5 \%$ among adults in the Nordic region, but large differences were seen between the five Nordic countries (Table 14).

Table 14: Ranking from lowest to highest proportion with an unhealthy diet among adults in the five Nordic countries. NORMO 2014

The proportion with an unhealthy diet was higher in Sweden than in the other Nordic countries. Furthermore, the proportion with an unhealthy diet was higher in Denmark and Iceland than in Finland and Norway.

Tables 15 and 16 show the population levels and the development from 2011 to 2014 in the proportion with an unhealthy diet and the intake of selected foods.

Between 2011 and 2014, the overall dietary quality score has decreased as the proportion with an unhealthy diet (dietary quality score $0-4$ ) increased and the proportion with a healthy diet (dietary quality score 9-12) decreased (Figure 1). 
Figure 1: Population levels and the development in dietary quality among adults in the Nordic region. NORMO 2011 and 2014

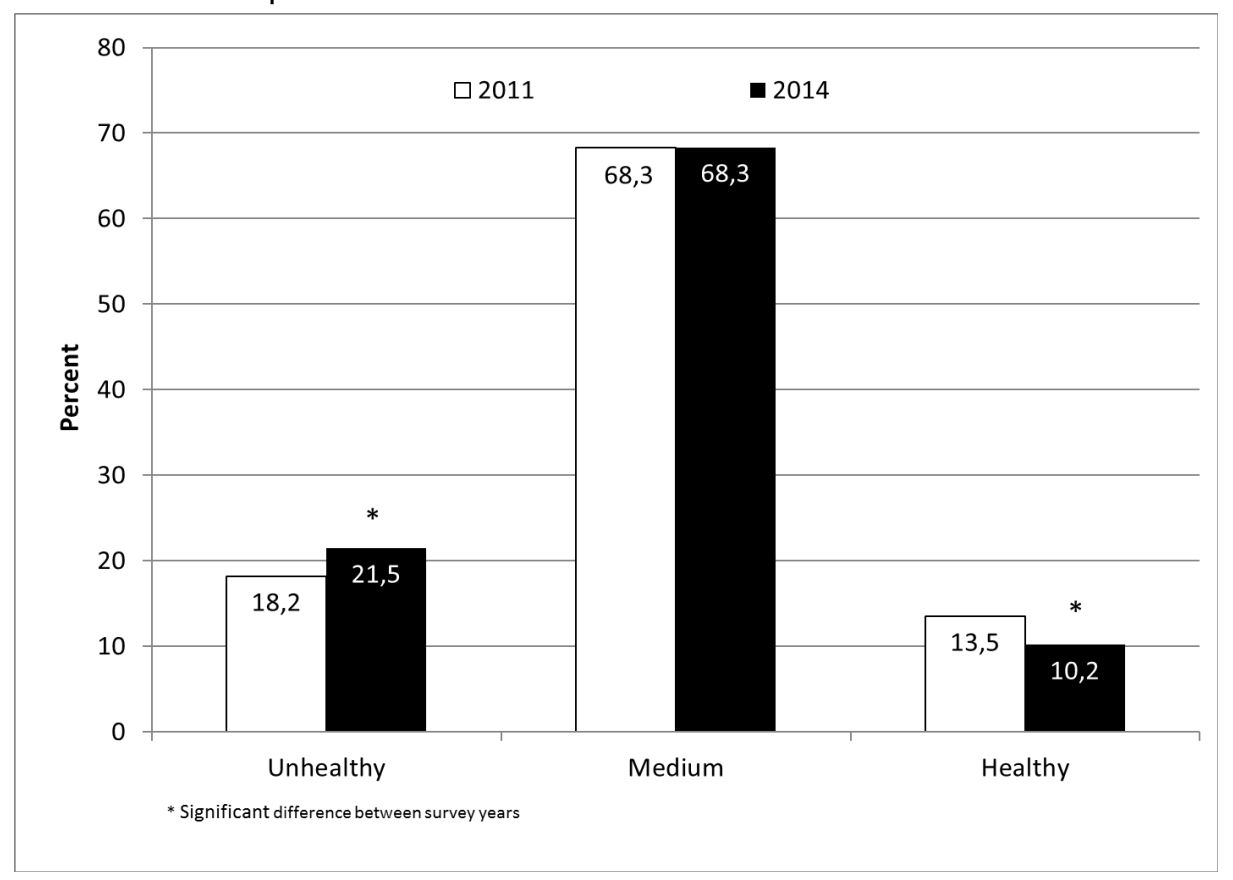

An increase in the proportion with an unhealthy diet was found in the Nordic region between 2011 and 2014, reflecting an increase in Sweden, Iceland and Norway. When analysing the overall dietary quality score, it showed a decrease in the Nordic region as well as in all the Nordic countries, except in Finland. In the Nordic region, an unfavorable development from 2011 to 2014 was seen for the intake of fish and whole grain bread (decreased) and the proportion with a high intake of foods rich in saturated fat (increased). The intake of whole grain bread decreased in all the Nordic countries, while the intake of fish decreased in Denmark and Norway. However, the decrease in the intake of fish was very small in the Nordic region. Furthermore, the proportion with a high consumption of foods rich in saturated fat increased in Sweden, Iceland and Norway.

The only favorable development among adults in the Nordic region was a decrease in the proportion with a high consumption of sugar-rich foods. This was also seen in Denmark, Finland and Norway while no change was found in Sweden and Iceland. When analysing the intake of fruit and vegetables in the Nordic region, no change was found but an increase was seen in Finland and Sweden and a decrease in Norway. 
Table 15: Mean and proportion (95\% Cl) for estimates of dietary intake among adults in the Nordic countries. NORMO 2011 and 2014

\begin{tabular}{|c|c|c|c|c|c|c|c|c|c|c|c|c|}
\hline & \multicolumn{2}{|c|}{ Denmark } & \multicolumn{2}{|c|}{ Finland } & \multicolumn{2}{|c|}{ Sweden } & \multicolumn{2}{|c|}{ Iceland } & \multicolumn{2}{|c|}{ Norway } & \multicolumn{2}{|c|}{ Nordic region@ } \\
\hline & $\begin{array}{r}2011 \\
(n=1,943)\end{array}$ & $\begin{array}{r}2014 \\
(n=1,969)\end{array}$ & $\begin{array}{r}2011 \\
(n=1,966)\end{array}$ & $\begin{array}{r}2014 \\
(n=1,561)\end{array}$ & $\begin{array}{r}2011 \\
(n=1,834)\end{array}$ & $\begin{array}{r}2014 \\
(n=1,700)\end{array}$ & $\begin{array}{r}2011 \\
(n=1,946)\end{array}$ & $\begin{array}{r}2014 \\
(n=1,916)\end{array}$ & $\begin{array}{r}2011 \\
(n=1,035)\end{array}$ & $\begin{array}{r}2014 \\
(n=961)\end{array}$ & $\begin{array}{r}2011 \\
(n=8,833)\end{array}$ & $\begin{array}{r}2014 \\
(n=8,144)\end{array}$ \\
\hline Dietary quality score & $\begin{array}{r}6.2 \\
(6.1 ; 6.3)\end{array}$ & $\begin{array}{r}6.0 * * \\
(5.9 ; 6.1)\end{array}$ & $\begin{array}{r}6.5 \\
(6.4 ; 6.6)\end{array}$ & $\begin{array}{r}6.5 \\
(6.4 ; 6.6)\end{array}$ & $\begin{array}{r}5.9 \\
(5.8 ; 6.0)\end{array}$ & $\begin{array}{r}5 \cdot 7^{* * *} \\
(5.6 ; 5.8)\end{array}$ & $\begin{array}{r}6.1 \\
(6.0 ; 6.2)\end{array}$ & $\begin{array}{r}5.8^{* * *} \\
(5.7 ; 5.8)\end{array}$ & $\begin{array}{r}7.0 \\
(6.9 ; 7.2)\end{array}$ & $\begin{array}{r}6.6 * * * \\
(6.4 ; 6.7)\end{array}$ & $\begin{array}{r}6.3 \\
(6.3 ; 6.4)\end{array}$ & $\begin{array}{r}6.1 * * * \\
(6.0 ; 6.1)\end{array}$ \\
\hline $\begin{array}{l}\text { High intake of sugar- } \\
\text { rich foods }(\%)\end{array}$ & $\begin{array}{r}36.7 \\
(34.7 ; 38.7)\end{array}$ & $\begin{array}{r}32.4^{*} \\
(30.3 ; 34.4)\end{array}$ & $\begin{array}{r}34.6 \\
(32.5 ; 36.6)\end{array}$ & $\begin{array}{r}30.3^{* *} \\
(28.1 ; 32.4)\end{array}$ & $\begin{array}{r}24.7 \\
22.8 ; 26.7)\end{array}$ & $\begin{array}{r}24.5 \\
(22.6 ; 26.5)\end{array}$ & $\begin{array}{r}42.1 \\
(39.9 ; 43.1)\end{array}$ & $\begin{array}{r}40.9 \\
(38.8 ; 43.1)\end{array}$ & $\begin{array}{r}25.8 \\
23.1 ; 28.4)\end{array}$ & $\begin{array}{r}21.7^{*} \\
19.2 ; 24.2)\end{array}$ & $\begin{array}{r}29.8 \\
(28.9 ; 30.8)\end{array}$ & $\begin{array}{r}27.1 * * * \\
(26.1 ; 28.0)\end{array}$ \\
\hline $\begin{array}{l}\text { High intake of foods } \\
\text { rich in sat. fat }{ }^{\ddagger}(\%)\end{array}$ & $\begin{array}{r}31.2 \\
(29.2 ; 33.1)\end{array}$ & $\begin{array}{r}28.3^{-} \\
(26.3 ; 30.3)\end{array}$ & $\begin{array}{r}30.7 \\
(28.6 ; 32.7)\end{array}$ & $\begin{array}{r}32.1 \\
(29.8 ; 34.3)\end{array}$ & $\begin{array}{r}46.4 \\
(44.1 ; 48.6)\end{array}$ & $\begin{array}{r}53 \cdot 5^{* * *} \\
(51.1 ; 55.8)\end{array}$ & $\begin{array}{r}32.0 \\
(30.0 ; 34.1)\end{array}$ & $\begin{array}{r}40.2 * * * \\
(38.0 ; 42.3)\end{array}$ & $\begin{array}{r}41.4 \\
(38.4 ; 44.3)\end{array}$ & $\begin{array}{r}47.5^{* * *} \\
(44.5 ; 50.6)\end{array}$ & $\begin{array}{r}38.6 \\
(37.6 ; 39.6)\end{array}$ & $\begin{array}{r}42.3^{* * *} \\
(41.2 ; 43.3)\end{array}$ \\
\hline $\begin{array}{l}\text { Fruits \& vegetables " } 5+ \\
\text { a day" }(\%)\end{array}$ & $\begin{array}{r}13.4 \\
(11.9 ; 14.8)\end{array}$ & $\begin{array}{r}13.0 \\
(11.5 ; 14.4)\end{array}$ & $\begin{array}{r}9.4 \\
(8.1 ; 10.6)\end{array}$ & $\begin{array}{r}12.9^{* * *} \\
(11.3 ; 14.5)\end{array}$ & $\begin{array}{r}12.5 \\
(11.0 ; 14.0)\end{array}$ & $\begin{array}{r}14.3 \\
(12.7 ; 15.9)\end{array}$ & $\begin{array}{r}8.7 \\
(7.4 ; 9.9)\end{array}$ & $\begin{array}{r}7.0^{-} \\
(5.9 ; 8.1)\end{array}$ & $\begin{array}{r}19.9 \\
(17.5 ; 22.3)\end{array}$ & $\begin{array}{l}10.4^{* * *} \\
(8.5 ; 12.2)\end{array}$ & $\begin{array}{r}13.4 \\
(12.7 ; 14.1)\end{array}$ & $\begin{array}{r}12.8 \\
(12.1 ; 13.6)\end{array}$ \\
\hline $\begin{array}{l}\text { Fish (main course } \\
\text { twice/week) (\%) }\end{array}$ & $\begin{array}{r}24.6 \\
(22.7 ; 26.5)\end{array}$ & $\begin{array}{r}21.7^{*} \\
(19.9 ; 23.5)\end{array}$ & $\begin{array}{r}33.7 \\
(31.7 ; 35.8)\end{array}$ & $\begin{array}{r}32.7 \\
(30.5 ; 35.0)\end{array}$ & $\begin{array}{r}35.6 \\
(33.5 ; 37.8)\end{array}$ & $\begin{array}{r}36.8 \\
(34.6 ; 39.0)\end{array}$ & $\begin{array}{r}64.5 \\
(62.4 ; 66.6)\end{array}$ & $\begin{array}{r}64.0 \\
(61.9 ; 66.1)\end{array}$ & $\begin{array}{r}60.4 \\
(57.5 ; 63.3)\end{array}$ & $\begin{array}{r}55.0^{*} \\
(52.0 ; 58.0)\end{array}$ & $\begin{array}{r}38.3 \\
(37.3 ; 39.3)\end{array}$ & $\begin{array}{r}36.7^{*} \\
(35.7 ; 37.7)\end{array}$ \\
\hline $\begin{array}{l}\text { Whole grain bread } \\
(\geq 50 \% \text { of daily bread in- } \\
\text { take) }(\%)\end{array}$ & $\begin{array}{r}97.2 \\
(96.5 ; 97.9)\end{array}$ & $\begin{array}{r}96.1^{-} \\
(95.3 ; 97.0)\end{array}$ & $\begin{array}{r}98.0 \\
(97.4 ; 98.7)\end{array}$ & $\begin{array}{r}95.6 * * * \\
(94.6 ; 96.6)\end{array}$ & $\begin{array}{r}92.1 \\
(90.9 ; 93.4)\end{array}$ & $\begin{array}{r}91.7 \\
(90.4 ; 93.1)\end{array}$ & $\begin{array}{r}97.7 \\
(97.1 ; 98.4)\end{array}$ & $\begin{array}{r}95 \cdot 3^{* * *} \\
(94.4 ; 96.3)\end{array}$ & $\begin{array}{r}97.2 \\
(96.2 ; 98.2)\end{array}$ & $\begin{array}{r}96.9 \\
(95.9 ; 98.0)\end{array}$ & $\begin{array}{r}95.5 \\
(95.1 ; 96.0)\end{array}$ & $\begin{array}{r}94.6 * * \\
(94.1 ; 95.1)\end{array}$ \\
\hline Unhealthy diet ${ }^{\mathrm{n}}(\%)$ & $\begin{array}{r}21.3 \\
(19.4 ; 23.1)\end{array}$ & $\begin{array}{r}22.7 \\
(20.9 ; 24.6)\end{array}$ & $\begin{array}{r}15.7 \\
(14.1 ; 17.3)\end{array}$ & $\begin{array}{r}15.4 \\
(13.6 ; 17.2)\end{array}$ & $\begin{array}{r}23.0 \\
(21.0 ; 24.9)\end{array}$ & $\begin{array}{r}27.5^{* *} \\
(25.4 i 29.6)\end{array}$ & $\begin{array}{r}19 \\
(17 \cdot 3 ; 20.7)\end{array}$ & $\begin{array}{r}24.5^{* * *} \\
(22.6 ; 26.4)\end{array}$ & $\begin{array}{r}8.8 \\
(7.1 ; 10.5)\end{array}$ & $\begin{array}{r}14.4^{* * *} \\
(12-2 ; 16.7)\end{array}$ & $\begin{array}{r}18.2 \\
(17.4 ; 19.0)\end{array}$ & $\begin{array}{r}21.5^{* * *} \\
(20.6 ; 22.4)\end{array}$ \\
\hline
\end{tabular}

$\quad \quad \quad-p<0.10,{ }^{*} p<0.05,{ }^{* *} p<0.01,{ }^{* * *} p<0.001$ Differences between survey years 2011 and 2014 using t-tests and Chi-square tests.

@Weighted according to population size in the five Nordic countries.

"Overall dietary quality score between o and 12 points based on intakes of fruits and vegetables, whole grain bread, fish as a main course, sugar-rich foods (chocolate/candy,

cakes, soft drinks), pommes frites, type of fat used on bread, full fat cheese and sausages.

${ }^{\mathrm{s}}$ Based on intakes of chocolate/candy, cakes and soft drinks.

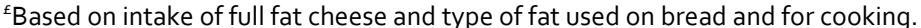

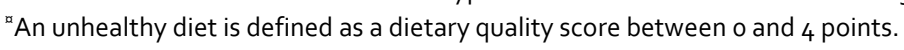


Table 16: Mean $(95 \% \mathrm{Cl})$ intake of selected foods among adults in the Nordic countries. NORMO 2011 and 2014

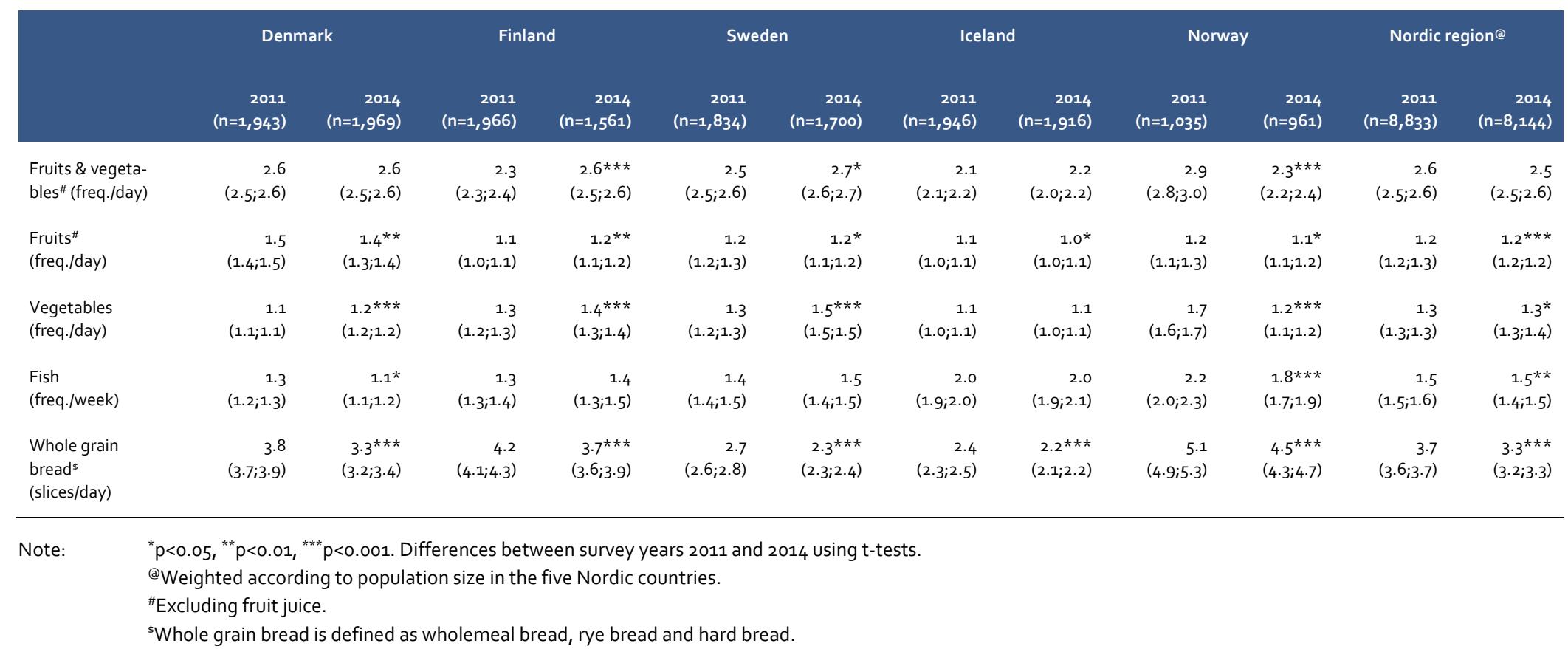




\subsubsection{Gender}

In 2014, the overall dietary quality score was lower and thus the proportion with an unhealthy diet higher in men than in women in the Nordic region (24.7\% vs. $18.4 \%$ ). This pattern was seen in all the Nordic countries, except Norway (Table 17 and 19). In the Nordic region, men had a lower intake of fruits, vegetables and fish than women, but a higher intake of wholegrain bread. However, the proportions eating at least half of their daily bread intake as whole grain bread were lower in men than women. Men consumed fewer healthy foods than women in the Nordic countries Men had a lower intake of fruits and vegetables than women in all countries. The intake of fish was lower in men than women in Finland and Norway, but higher in men than women in Iceland. Men had a higher intake of whole grain bread than women in all countries except Denmark. The proportions with a high consumption of sugar-rich foods and foods rich in saturated fat were higher in men than in women.

Between 2011 and 2014, the proportion with an unhealthy diet increased both in men and women in the Nordic region. The proportion with an unhealthy diet increased more in men than in women. In men, the proportions with a high consumption of sugar-rich foods, intake of fish and whole grain bread decreased from 2011 to 2014 while the proportion with a high consumption of foods rich in saturated fat increased. An increase in the proportion with a high consumption of foods rich in saturated fat was seen among men in Sweden, Iceland and Norway and among women in Iceland while a decrease was seen among men in Denmark. In women, the intake of fruits and vegetables and whole grain bread decreased in the Nordic region, but the decrease in whole grain bread intake was very small. The proportion with a high consumption of sugar-rich foods decreased in women in the Nordic region and in women in Finland. For other foods, mixed results of the development from 2011 to 2014 were found for men and women (Table 17-20). 
Table 17: Mean and proportion $(95 \% \mathrm{Cl}$ ) for estimates of dietary intake among men in the Nordic countries. NORMO 2011 and 2014

\begin{tabular}{|c|c|c|c|c|c|c|c|c|c|c|c|c|}
\hline & \multicolumn{2}{|c|}{ Denmark } & \multicolumn{2}{|c|}{ Finland } & \multicolumn{2}{|c|}{ Sweden } & \multicolumn{2}{|c|}{ Iceland } & \multicolumn{2}{|c|}{ Norway } & \multicolumn{2}{|c|}{ Nordic region@ } \\
\hline & $\begin{array}{r}2011 \\
(n=904)\end{array}$ & $\begin{array}{r}2014 \\
(n=955)\end{array}$ & $\begin{array}{r}2011 \\
(n=898)\end{array}$ & $\begin{array}{r}2014 \\
(n=679)\end{array}$ & $\begin{array}{r}2011 \\
(n=864)\end{array}$ & $\begin{array}{r}2014 \\
(n=785)\end{array}$ & $\begin{array}{r}2011 \\
(n=935)\end{array}$ & $\begin{array}{r}2014 \\
(\mathrm{n}=867)\end{array}$ & $\begin{array}{r}2011 \\
(n=502)\end{array}$ & $\begin{array}{r}2014 \\
(n=436)\end{array}$ & $\begin{array}{r}2011 \\
(n=4,121)\end{array}$ & $\begin{array}{r}2014 \\
(n=3,733)\end{array}$ \\
\hline Dietary quality score ${ }^{\#}$ & $\begin{array}{r}5.9 \\
(5.8 ; 6.0)\end{array}$ & $\begin{array}{r}5.7^{*} \\
(5.6 ; 5 \cdot 9)\end{array}$ & $\begin{array}{r}6.4 \\
(6.3 ; 6.5)\end{array}$ & $\begin{array}{r}6.3 \\
(6.1 ; 6.4)\end{array}$ & $\begin{array}{r}5.7 \\
(5.6 ; 5.9)\end{array}$ & $\begin{array}{r}5.5^{*} \\
(5.4 ; 5.7)\end{array}$ & $\begin{array}{r}5.9 \\
(5.8 ; 6.1)\end{array}$ & $\begin{array}{l}5.6 * * * \\
(5.5 ; 5.7)\end{array}$ & $\begin{array}{r}6.9 \\
(6.8 ; 7.1)\end{array}$ & $\begin{array}{r}6.4^{* * *} \\
(6.2 ; 6.6)\end{array}$ & $\begin{array}{r}6.2 \\
(6.1 ; 6.2)\end{array}$ & $\begin{array}{r}5.9^{* * *} \\
(5.8 ; 5.9)\end{array}$ \\
\hline $\begin{array}{l}\text { High intake of sugar- } \\
\text { rich foods }(\%)\end{array}$ & $\begin{array}{r}41.4 \\
(38.3 ; 44.4)\end{array}$ & $\begin{array}{r}35.2^{*} \\
(32.2 ; 38.2)\end{array}$ & $\begin{array}{r}33.1 \\
(30.1 ; 36.2)\end{array}$ & $\begin{array}{r}30.1 \\
(26.8 ; 33.4)\end{array}$ & $\begin{array}{r}26.0 \\
(23.2 ; 28.9)\end{array}$ & $\begin{array}{r}26.9 \\
23.9 ; 30.0)\end{array}$ & $\begin{array}{r}49.2 \\
(46.0 ; 52.4)\end{array}$ & $\begin{array}{r}45.4 \\
(45.2 ; 48.7)\end{array}$ & $\begin{array}{r}29.0 \\
(25.1 ; 32.9)\end{array}$ & $\begin{array}{r}21.8^{*} \\
(18.1 ; 25.6)\end{array}$ & $\begin{array}{r}31.7 \\
(30.3 ; 33.1)\end{array}$ & $\begin{array}{r}28.7^{*} \\
(27.3 ; 30.1)\end{array}$ \\
\hline $\begin{array}{l}\text { High intake of foods } \\
\text { rich in sat. fat }{ }^{ \pm}(\%)\end{array}$ & $\begin{array}{r}36.5 \\
(33.5 ; 39.4)\end{array}$ & $\begin{array}{r}31.6 * \\
(28.7 ; 34.5)\end{array}$ & $\begin{array}{r}31.7 \\
(28.6 ; 34.8)\end{array}$ & $\begin{array}{r}35.9 \\
(32.4 ; 39.4)\end{array}$ & $\begin{array}{r}45 \cdot 3 \\
(41.9 ; 48.9)\end{array}$ & $\begin{array}{r}55.7^{* * * *} \\
(52 \cdot 3 ; 59.1)\end{array}$ & $\begin{array}{r}31.9 \\
(28.9 ; 35.0)\end{array}$ & $\begin{array}{r}42.2^{* * *} \\
(38.9 ; 45.5)\end{array}$ & $\begin{array}{r}41.7 \\
(37.4 ; 46.0)\end{array}$ & $\begin{array}{r}52.6 * * \\
(48.1 ; 57.2)\end{array}$ & $\begin{array}{r}39.7 \\
(38.3 ; 41.2)\end{array}$ & $\begin{array}{r}45 \cdot 5^{* * *} \\
(43 \cdot 9 ; 47 \cdot 1)\end{array}$ \\
\hline $\begin{array}{l}\text { Fruits \& vegetables } \\
\text { " } 5+\text { a day" (\%) }\end{array}$ & $\begin{array}{r}10.3 \\
(8.4 ; 12.2)\end{array}$ & $\begin{array}{r}9.9 \\
(8.1 ; 11.8)\end{array}$ & $\begin{array}{r}6.7 \\
(5.1 ; 8.4)\end{array}$ & $\begin{array}{r}7.3 \\
(5.4 ; 9.1)\end{array}$ & $\begin{array}{r}8.5 \\
(6.7 ; 10.3)\end{array}$ & $\begin{array}{r}9.8 \\
(7.7 ; 11.8)\end{array}$ & $\begin{array}{r}5.8 \\
(4.377 .2)\end{array}$ & $\begin{array}{r}4.6 \\
(3.3 ; 6.0)\end{array}$ & $\begin{array}{r}16.6 \\
(13 \cdot 3 ; 19.8)\end{array}$ & $\begin{array}{r}6.9^{* * *} \\
(4.6 ; 9 \cdot 2)\end{array}$ & $\begin{array}{r}10.1 \\
(9.2 ; 11.0)\end{array}$ & $\begin{array}{r}8.7^{*} \\
(7.8 ; 9.6)\end{array}$ \\
\hline $\begin{array}{l}\text { Fish (main course } \\
\text { twice/week) (\%) }\end{array}$ & $\begin{array}{r}22.8 \\
(20.1 ; 25.5)\end{array}$ & $\begin{array}{r}20.0 \\
(17.5 ; 22.5)\end{array}$ & $\begin{array}{r}31.0 \\
(28.0 ; 34.0)\end{array}$ & $\begin{array}{r}29.8 \\
(26.6 ; 33.1)\end{array}$ & $\begin{array}{r}33.8 \\
(30.3 ; 36.9)\end{array}$ & $\begin{array}{r}35.2 \\
(32.0 ; 38.5)\end{array}$ & $\begin{array}{r}66.4 \\
(63.4 ; 69.4)\end{array}$ & $\begin{array}{r}64.2 \\
(61.0 ; 67.3)\end{array}$ & $\begin{array}{r}61.5 \\
(57 \cdot 3 ; 65.7)\end{array}$ & $\begin{array}{r}50.8^{* * * *} \\
(46.4 ; 55 \cdot 3)\end{array}$ & $\begin{array}{r}37.1 \\
(35.6 ; 38.5)\end{array}$ & $\begin{array}{r}34.1^{\text {** }} \\
(32.7 ; 35.6)\end{array}$ \\
\hline $\begin{array}{l}\text { Whole grain bread ( } \geq \\
50 \% \text { of daily bread in- } \\
\text { take) (\%) }\end{array}$ & $\begin{array}{r}96.5 \\
(95.3 ; 97.6)\end{array}$ & $\begin{array}{r}94.9^{-} \\
(93.5 ; 96.3)\end{array}$ & $\begin{array}{r}96.9 \\
(95.8 ; 98.0)\end{array}$ & $\begin{array}{r}94.5^{*} \\
(92.8 ; 96.2)\end{array}$ & $\begin{array}{r}90.0 \\
(88.0 ; 92.0)\end{array}$ & $\begin{array}{r}89.2 \\
(87.0 ; 91.3)\end{array}$ & $\begin{array}{r}96.9 \\
(95.8 ; 98.0)\end{array}$ & $\begin{array}{r}93.0 * * * \\
(91.3 ; 94.7)\end{array}$ & $\begin{array}{r}97.4 \\
(96.0 ; 98.8)\end{array}$ & $\begin{array}{r}96.1 \\
(94.2 ; 97.9)\end{array}$ & $\begin{array}{r}94.4 \\
(93.7 ; 95.1)\end{array}$ & $\begin{array}{r}92.9^{* *} \\
(92.1 ; 93.7)\end{array}$ \\
\hline Unhealthy $\operatorname{diet}^{\mathrm{I}}(\%)$ & $\begin{array}{r}24.5 \\
(21.7 ; 27.4)\end{array}$ & $\begin{array}{r}27.5 \\
(24.7 ; 30.3)\end{array}$ & $\begin{array}{r}18.2 \\
(15.7 ; 20.7)\end{array}$ & $\begin{array}{r}18.5 \\
(15.6 ; 21.4)\end{array}$ & $\begin{array}{r}24.8 \\
(21.9 ; 27.6)\end{array}$ & $\begin{array}{r}29.7^{*} \\
(26.6 ; 32.9)\end{array}$ & $\begin{array}{r}21.5 \\
(18.9 ; 24.2)\end{array}$ & $\begin{array}{r}27.5^{* *} \\
(24.5 ; 30.5)\end{array}$ & $\begin{array}{r}8.2 \\
(5.8 ; 10.6)\end{array}$ & $\begin{array}{r}17.1^{* * *} \\
(13.5 ; 20.7)\end{array}$ & $\begin{array}{r}19.9 \\
(18.7 ; 21.1)\end{array}$ & $\begin{array}{r}24.7 * k * \\
(23.3 ; 26.1)\end{array}$ \\
\hline
\end{tabular}

$\quad \quad \quad-p<0.10,{ }^{*} p<0.05,{ }^{* *} p<0.01,{ }^{* * *} p<0.001$. Differences between survey years 2011 and 2014 using t-tests and Chi-square tests.

¿Weighted according to population size in the five Nordic countries.

\#Overall dietary quality score between o and 12 points based on intakes of fruits and vegetables, whole grain bread, fish as a main course, sugar-rich foods (choco-

late/candy, cakes, soft drinks), pommes frites, type of fat used on bread, full fat cheese and sausages.

'Based on intakes of chocolate/candy, cakes and soft drinks.

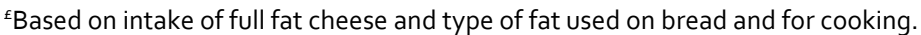

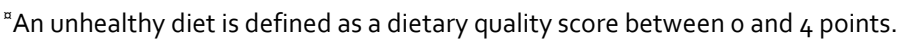


Table 18: Mean ( $95 \% \mathrm{Cl}$ ) intake of selected foods among men in the Nordic countries. NORMO 2011 and 2014

\begin{tabular}{|c|c|c|c|c|c|c|c|c|c|c|c|c|}
\hline & \multicolumn{2}{|c|}{ Denmark } & \multicolumn{2}{|c|}{ Finland } & \multicolumn{2}{|c|}{ Sweden } & \multicolumn{2}{|c|}{ Iceland } & \multicolumn{2}{|c|}{ Norway } & \multicolumn{2}{|c|}{ Nordic region@ } \\
\hline & $\begin{array}{r}2011 \\
(n=904)\end{array}$ & $\begin{array}{r}2014 \\
(n=955)\end{array}$ & $\begin{array}{r}2011 \\
(n=898)\end{array}$ & $\begin{array}{r}2014 \\
(n=679)\end{array}$ & $\begin{array}{r}2011 \\
(n=864)\end{array}$ & $\begin{array}{r}2014 \\
(n=785)\end{array}$ & $\begin{array}{r}2011 \\
(n=935)\end{array}$ & $\begin{array}{r}2014 \\
(n=867)\end{array}$ & $\begin{array}{r}2011 \\
(n=502)\end{array}$ & $\begin{array}{r}2014 \\
(n=436)\end{array}$ & $\begin{array}{r}2011 \\
(n=4,121)\end{array}$ & $\begin{array}{r}2014 \\
(n=3,733)\end{array}$ \\
\hline $\begin{array}{l}\text { Fruits \& vegetables } \\
\text { (freq./day) }\end{array}$ & $\begin{array}{r}2.3 \\
(2.2 ; 2.4)\end{array}$ & $\begin{array}{r}2.3 \\
(2.2 ; 2.3)\end{array}$ & $\begin{array}{r}2.0 \\
(1.9 ; 2.1)\end{array}$ & $\begin{array}{r}2.1 \\
(2.0 ; 2.2)\end{array}$ & $\begin{array}{r}2.1 \\
(2.1 ; 2.2)\end{array}$ & $\begin{array}{r}2.4^{* * *} \\
(2.3 ; 2.5)\end{array}$ & $\begin{array}{r}1.9 \\
(1.8 ; 2.0)\end{array}$ & $\begin{array}{r}1.9 \\
(1.8 ; 1.9)\end{array}$ & $\begin{array}{r}2.5 \\
(2.3 ; 2.6)\end{array}$ & $\begin{array}{r}2.0 * * * \\
(1.9 ; 2.2)\end{array}$ & $\begin{array}{r}2.2 \\
(2.2 ; 2.2)\end{array}$ & $\begin{array}{r}2.2 \\
(2.2 ; 2.3)\end{array}$ \\
\hline $\begin{array}{l}\text { Fruits }^{\#} \\
\text { (freq./day) }\end{array}$ & $\begin{array}{r}1.3 \\
(1.2 ; 1.4)\end{array}$ & $\begin{array}{r}1.2^{*} \\
(1.1 ; 1.3)\end{array}$ & $\begin{array}{r}0.9 \\
(0.8 ; 0.9)\end{array}$ & $\begin{array}{r}0.9 \\
(0.8 ; 1.0)\end{array}$ & $\begin{array}{r}1.0 \\
(0.9 ; 1.1)\end{array}$ & $\begin{array}{r}1.0 \\
(0,9 ; 1.1)\end{array}$ & $\begin{array}{r}0.9 \\
(0.9 ; 1.0)\end{array}$ & $\begin{array}{r}0.9 \\
(0.8 ; 0.9)\end{array}$ & $\begin{array}{r}1.1 \\
(1.0 ; 1.2)\end{array}$ & $\begin{array}{r}1.0^{-} \\
(0.9 ; 1.0)\end{array}$ & $\begin{array}{r}1.1 \\
(1.0 ; 1.1)\end{array}$ & $\begin{array}{r}1.0^{-} \\
(1.0 ; 1.1)\end{array}$ \\
\hline $\begin{array}{l}\text { Vegetables } \\
\text { (freq./day) }\end{array}$ & $\begin{array}{r}0.9 \\
(0.9 ; 1.0)\end{array}$ & $\begin{array}{r}1.1 * * \\
(1.0 ; 1.1)\end{array}$ & $\begin{array}{r}1.1 \\
(1.0 ; 1.1)\end{array}$ & $\begin{array}{r}1.2^{-} \\
(1.1 ; 1.2)\end{array}$ & $\begin{array}{r}1.1 \\
(1.1 ; 1.2)\end{array}$ & $\begin{array}{l}1.4^{* * *} \\
(1.3 ; 1.4)\end{array}$ & $\begin{array}{r}0.9 \\
(0.9 ; 1.0)\end{array}$ & $\begin{array}{r}1.0 \\
(0.9 ; 1.0)\end{array}$ & $\begin{array}{r}1.4 \\
(1.3 ; 1.5)\end{array}$ & $\begin{array}{r}1.1 * * * \\
(1.0 ; 1.1)\end{array}$ & $\begin{array}{r}1.1 \\
(1.1 ; 1.2)\end{array}$ & $\begin{array}{r}1.2^{* *} \\
(1.2 ; 1.2)\end{array}$ \\
\hline $\begin{array}{l}\text { Fish } \\
\text { (freq./week) }\end{array}$ & $\begin{array}{r}1.2 \\
(1.1 ; 1.3)\end{array}$ & $\begin{array}{r}1.1^{*} \\
(1.0 ; 1.2)\end{array}$ & $\begin{array}{r}1.2 \\
(1.2 ; 1.3)\end{array}$ & $\begin{array}{r}1.3 \\
(1.2 ; 1.4)\end{array}$ & $\begin{array}{r}1.3 \\
(1.3 ; 1.4)\end{array}$ & $\begin{array}{r}1.4 \\
(1.3 ; 1.5)\end{array}$ & $\begin{array}{r}2.0 \\
(2.0 ; 2.1)\end{array}$ & $\begin{array}{r}2.1 \\
(2.0 ; 2.2)\end{array}$ & $\begin{array}{r}2.2 \\
(2.0 ; 2.4)\end{array}$ & $\begin{array}{r}1.7^{* * *} \\
(1.6 ; 1.8)\end{array}$ & $\begin{array}{r}1.5 \\
(1.4 ; 1.5)\end{array}$ & $\begin{array}{r}1.4^{* *} \\
(1.3 ; 1.4)\end{array}$ \\
\hline $\begin{array}{l}\text { Whole grain bread } \\
\text { (slices/day) }\end{array}$ & $\begin{array}{r}4 \cdot 4 \\
(4 \cdot 2 ; 4 \cdot 5)\end{array}$ & $\begin{array}{l}3 \cdot 4^{* * *} \\
(3.2 ; 3 \cdot 5)\end{array}$ & $\begin{array}{r}4.7 \\
(4.5 ; 4.9)\end{array}$ & $\begin{array}{r}4 \cdot 4^{*} \\
(4 \cdot 2 ; 4 \cdot 6)\end{array}$ & $\begin{array}{r}3.0 \\
(2.8 ; 3.2)\end{array}$ & $\begin{array}{r}2.7^{* *} \\
(2.5 ; 2.8)\end{array}$ & $\begin{array}{r}2.7 \\
(2.6 ; 2.9)\end{array}$ & $\begin{array}{r}2.4^{* * *} \\
(2.2: 2.5)\end{array}$ & $\begin{array}{r}6.0 \\
(5.6 ; 6.3)\end{array}$ & $\begin{array}{r}5.2^{* * *} \\
(4.9 ; 5 \cdot 5)\end{array}$ & $\begin{array}{r}4 \cdot 2 \\
(4 \cdot 1 ; 4 \cdot 3)\end{array}$ & $\begin{array}{l}3.6 * * * \\
(3.6 ; 3.7)\end{array}$ \\
\hline
\end{tabular}

$\quad \quad$ Note: $\quad-p<0.10,{ }^{*} p<0.05,{ }^{* *} p<0.01,{ }^{* * *} p<0.001$. Differences between survey years 2011 and 2014 using t-tests.

@Weighted according to population size in the five Nordic countries.

\#Excluding fruit juice.

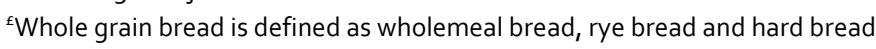


Table 19: Mean and proportion ( $95 \% \mathrm{Cl}$ ) for estimates of dietary intake among women in the Nordic countries. NORMO 2011 and 2014

\begin{tabular}{|c|c|c|c|c|c|c|c|c|c|c|c|c|}
\hline & \multicolumn{2}{|c|}{ Denmark } & \multicolumn{2}{|c|}{ Finland } & \multicolumn{2}{|c|}{ Sweden } & \multicolumn{2}{|c|}{ Iceland } & \multicolumn{2}{|c|}{ Norway } & \multicolumn{2}{|c|}{ Nordic region@ } \\
\hline & $\begin{array}{r}2011 \\
(n=1,039)\end{array}$ & $\begin{array}{r}2014 \\
(n=1,019)\end{array}$ & $\begin{array}{r}2011 \\
(n=1,050)\end{array}$ & $\begin{array}{r}2014 \\
(n=876)\end{array}$ & $\begin{array}{r}2011 \\
(n=970)\end{array}$ & $\begin{array}{r}2014 \\
(n=915)\end{array}$ & $\begin{array}{r}2011 \\
(n=1,071)\end{array}$ & $\begin{array}{r}2014 \\
(n=1,095)\end{array}$ & $\begin{array}{r}2011 \\
(n=533)\end{array}$ & $\begin{array}{r}2014 \\
(n=525)\end{array}$ & $\begin{array}{r}2011 \\
(n=4,712)\end{array}$ & $\begin{array}{r}2014 \\
(n=4,408)\end{array}$ \\
\hline Dietary quality score ${ }^{\#}$ & $\begin{array}{r}6.4 \\
(6.3 ; 6.5)\end{array}$ & $\begin{array}{r}6.2^{*} \\
(6.1 ; 6.3)\end{array}$ & $\begin{array}{r}6.6 \\
(6.5 ; 6.8)\end{array}$ & $\begin{array}{r}6.7 \\
(6.6 ; 6.8)\end{array}$ & $\begin{array}{r}6.1 \\
(6.0 ; 6.2)\end{array}$ & $\begin{array}{r}5.9^{* *} \\
(5.7 ; 6.0)\end{array}$ & $\begin{array}{r}6.2 \\
(6.1 ; 6.3)\end{array}$ & $\begin{array}{r}5.9 * * * \\
(5.8 ; 6.0)\end{array}$ & $\begin{array}{r}7.2 \\
(7.0 ; 7 \cdot 3)\end{array}$ & $\begin{array}{r}6.7^{* * * *} \\
(6.5 ; 6.8)\end{array}$ & $\begin{array}{r}6.5 \\
(6.4 ; 6.5)\end{array}$ & $\begin{array}{r}6.3^{* * *} \\
(6.2 ; 6.3)\end{array}$ \\
\hline $\begin{array}{l}\text { High intake of sugar- } \\
\text { rich foods }(\%)\end{array}$ & $\begin{array}{r}31.9 \\
(29.3 ; 34.6)\end{array}$ & $\begin{array}{r}29.4 \\
(26.6 ; 32.2)\end{array}$ & $\begin{array}{r}35.6 \\
(33.1 ; 36.8)\end{array}$ & $\begin{array}{r}30.5^{* *} \\
(27.5 ; 33.4)\end{array}$ & $\begin{array}{r}23.4 \\
(20.8 ; 26.0)\end{array}$ & $\begin{array}{r}22.1 \\
(19.5 ; 24.7)\end{array}$ & $\begin{array}{r}34.8 \\
(32.0 ; 37.7)\end{array}$ & $\begin{array}{r}36.4 \\
(33.5 ; 39.2)\end{array}$ & $\begin{array}{r}22.4 \\
(18.9 ; 25.9)\end{array}$ & $\begin{array}{r}21.5 \\
(18.1 ; 24.9)\end{array}$ & $\begin{array}{r}27.9 \\
(26.7 ; 29.2)\end{array}$ & $\begin{array}{r}25.5^{*} \\
(24.2 ; 26.8)\end{array}$ \\
\hline $\begin{array}{l}\text { High intake of foods } \\
\text { rich in sat. fat }{ }^{\ddagger}(\%)\end{array}$ & $\begin{array}{r}25.8 \\
(23 \cdot 3 ; 28.4)\end{array}$ & $\begin{array}{r}25.0 \\
(22.3 ; 27.6)\end{array}$ & $\begin{array}{r}29.7 \\
(27.0 ; 32.5)\end{array}$ & $\begin{array}{r}28.6^{-} \\
(25.6 ; 31.5)\end{array}$ & $\begin{array}{r}47.4 \\
(44.3 ; 50.5)\end{array}$ & $\begin{array}{r}51.2 \\
(48.0 ; 54.4)\end{array}$ & $\begin{array}{r}32.1 \\
(29.3334 .9)\end{array}$ & $\begin{array}{r}38.2 * * * \\
(35 \cdot 3 ; 41.0)\end{array}$ & $\begin{array}{r}41.1 \\
(36.9 ; 45.2)\end{array}$ & $\begin{array}{r}42.5 \\
(38.5 ; 46.6)\end{array}$ & $\begin{array}{r}37.5 \\
(36.1 ; 38.9)\end{array}$ & $\begin{array}{r}39.1 * * \\
(37.6 ; 40.5)\end{array}$ \\
\hline $\begin{array}{l}\text { Fruits \& vegetables } \\
\text { " } 5+\text { a day" }(\%)\end{array}$ & $\begin{array}{r}16.5 \\
(14.3 ; 18.6)\end{array}$ & $\begin{array}{r}16.0 \\
(13.8 ; 18.3)\end{array}$ & $\begin{array}{r}11.8 \\
(9.9 ; 13.7)\end{array}$ & $\begin{array}{r}18.2 * * * \\
(15.7 ; 20.6)\end{array}$ & $\begin{array}{r}16.6 \\
(14.3 ; 18.9)\end{array}$ & $\begin{array}{r}18.9 \\
(16.4 ; 21.3)\end{array}$ & $\begin{array}{r}11.6 \\
(9.7 ; 13.5)\end{array}$ & $\begin{array}{r}9.4^{-} \\
(7.7 ; 11.1)\end{array}$ & $\begin{array}{r}23.4 \\
(19.9 ; 27.0)\end{array}$ & $\begin{array}{r}13.9^{* * *} \\
(11.0 ; 16.7)\end{array}$ & $\begin{array}{r}16.7 \\
(15.7 ; 17.8)\end{array}$ & $\begin{array}{r}17.0 \\
(15.9 ; 18.1)\end{array}$ \\
\hline $\begin{array}{l}\text { Fish (main course } \\
\text { twice/week) (\%) }\end{array}$ & $\begin{array}{r}26.4 \\
(23.8 ; 29.1)\end{array}$ & $\begin{array}{r}23.5 \\
(20.9 ; 26.1)\end{array}$ & $\begin{array}{r}36.3 \\
(33.5 ; 39.2)\end{array}$ & $\begin{array}{r}35.5 \\
(32.4 ; 38.5)\end{array}$ & $\begin{array}{r}37.5 \\
(34.5 ; 40.5)\end{array}$ & $\begin{array}{r}38.4 \\
(35.4 ; 41.5)\end{array}$ & $\begin{array}{r}62.8 \\
(59.7 ; 65.5)\end{array}$ & $\begin{array}{r}63.9 \\
(61.0 ; 66.7)\end{array}$ & $\begin{array}{r}59.2 \\
(55.1 ; 63.3)\end{array}$ & $\begin{array}{r}59.2 \\
(55.1 ; 63.2)\end{array}$ & $\begin{array}{r}39.5 \\
(38.1 ; 40.9)\end{array}$ & $\begin{array}{r}39.2 \\
(37.8 ; 40.6\end{array}$ \\
\hline $\begin{array}{l}\text { Whole grain bread ( } \geq \\
50 \% \text { of daily bread in- } \\
\text { take) (\%) }\end{array}$ & $\begin{array}{r}97.8 \\
(97.0 ; 98.7)\end{array}$ & $\begin{array}{r}97.3 \\
(96.3 ; 98.3)\end{array}$ & $\begin{array}{r}99.2 \\
(98.6 ; 99.7)\end{array}$ & $\begin{array}{r}96.6 * * * \\
(95.4 ; 97.8)\end{array}$ & $\begin{array}{r}94.3 \\
(92.8 ; 95.8)\end{array}$ & $\begin{array}{r}94.4 \\
(92.9 ; 95.9)\end{array}$ & $\begin{array}{r}98.6 \\
(97.9 ; 99.3)\end{array}$ & $\begin{array}{r}97.7 \\
(96.8 ; 98.6)\end{array}$ & $\begin{array}{r}97.0 \\
(95.6 ; 98.4)\end{array}$ & $\begin{array}{r}97.8 \\
(96.5 ; 99.0)\end{array}$ & $\begin{array}{r}96.7 \\
(96.2 ; 97.2)\end{array}$ & $\begin{array}{r}\text { } \\
96.2 \\
(95.7 ; 96.8)\end{array}$ \\
\hline Unhealthy diet ${ }^{\mathrm{x}}(\%)$ & $\begin{array}{r}17.9 \\
(15.6 ; 20.3)\end{array}$ & $\begin{array}{r}17.9 \\
(15.5 ; 20.2)\end{array}$ & $\begin{array}{r}13.3 \\
(11.3 ; 15.4)\end{array}$ & $\begin{array}{r}12.6 \\
(10.4 ; 14.7)\end{array}$ & $\begin{array}{r}21.1 \\
(18.6 ; 23.7)\end{array}$ & $\begin{array}{r}25.2^{*} \\
(22.5 ; 28.0)\end{array}$ & $\begin{array}{r}16.5 \\
(14.2 ; 18.7)\end{array}$ & $\begin{array}{r}21.4^{* *} \\
(19.0 ; 23.9)\end{array}$ & $\begin{array}{r}9.4 \\
(7.0 ; 11.9)\end{array}$ & $\begin{array}{r}11.8 \\
(9.1 ; 14.6)\end{array}$ & $\begin{array}{r}16.4 \\
(15.4 ; 17.5)\end{array}$ & $\begin{array}{r}18.4^{*} \\
(17.2 ; 19.5)\end{array}$ \\
\hline
\end{tabular}

Note: $\quad-p<0.10,{ }^{*} p<0.05,{ }^{* *} p<0.01,{ }^{* * *} p<0.001$. Differences between survey years 2011 and 2014 using t-tests and Chi-square tests.

@Weighted according to population size in the five Nordic countries.

\#Overall dietary quality score between o and 12 points based on intakes of fruits and vegetables, whole grain bread, fish as a main course, sugar-rich foods (chocolate/candy, cakes, soft drinks), pommes frites, type of fat used on bread, full fat cheese and sausages.

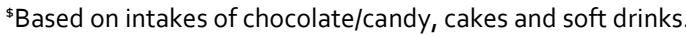

EBased on intake of full fat cheese and type of fat used on bread and for cooking.

${ }^{\text {a }} \mathrm{An}$ unhealthy diet is defined as a dietary quality score between $\mathrm{o}$ and 4 points. 
Table 20: Mean $(95 \% \mathrm{Cl})$ intake of selected foods among women in the Nordic countries. NORMO 2011 and 2014

\begin{tabular}{|c|c|c|c|c|c|c|c|c|c|c|c|c|}
\hline & \multicolumn{2}{|c|}{ Denmark } & \multicolumn{2}{|c|}{ Finland } & \multicolumn{2}{|c|}{ Sweden } & \multicolumn{2}{|c|}{ Iceland } & \multicolumn{2}{|c|}{ Norway } & \multicolumn{2}{|c|}{ Nordic region@ } \\
\hline & $\begin{array}{r}2011 \\
(n=1,039)\end{array}$ & $\begin{array}{r}2014 \\
(n=1,019)\end{array}$ & $\begin{array}{r}2011 \\
(n=1,050)\end{array}$ & $\begin{array}{r}2014 \\
(\mathrm{n}=876)\end{array}$ & $\begin{array}{r}2011 \\
(n=970)\end{array}$ & $\begin{array}{r}2014 \\
(n=915)\end{array}$ & $\begin{array}{r}2011 \\
(n=1,071)\end{array}$ & $\begin{array}{r}2014 \\
(n=1,095)\end{array}$ & $\begin{array}{r}2011 \\
(n=533)\end{array}$ & $\begin{array}{r}2014 \\
(n=525)\end{array}$ & $\begin{array}{r}2011 \\
(n=4,712)\end{array}$ & $\begin{array}{r}2014 \\
(n=4,408)\end{array}$ \\
\hline $\begin{array}{l}\text { Fruits \& vegeta- } \\
\text { bles" }^{\#} \text { (freq./d) }\end{array}$ & $\begin{array}{r}2.9 \\
(2.8 ; 3.0)\end{array}$ & $\begin{array}{r}2.9 \\
(2.8 ; 3.0)\end{array}$ & $\begin{array}{r}2.7 \\
(2.6 ; 2.8)\end{array}$ & $\begin{array}{r}3.0^{* * *} \\
(2.9 ; 3.1)\end{array}$ & $\begin{array}{r}2.9 \\
(2.8 ; 3.0)\end{array}$ & $\begin{array}{r}3.0 \\
(2.9 ; 3.1)\end{array}$ & $\begin{array}{r}2.4 \\
(2.3 ; 2.5)\end{array}$ & $\begin{array}{r}2.3 \\
(2.3 ; 2.4)\end{array}$ & $\begin{array}{r}3.3 \\
(3.1 ; 3.4)\end{array}$ & $\begin{array}{r}2.5^{* * *} \\
(2.4 ; 2.7)\end{array}$ & $\begin{array}{r}2.9 \\
(2.9 ; 3.0)\end{array}$ & $\begin{array}{r}2.9^{*} \\
(2.8 ; 2.9)\end{array}$ \\
\hline $\begin{array}{l}\text { Fruits }{ }^{\#} \\
\text { (freq./d) }\end{array}$ & $\begin{array}{r}1.6 \\
(1.6 ; 1.7)\end{array}$ & $\begin{array}{r}1.5^{*} \\
(1.4 ; 1.6)\end{array}$ & $\begin{array}{r}1.3 \\
(1.2: 1.3)\end{array}$ & $\begin{array}{r}1.4^{* * *} \\
(1.3 ; 1.5)\end{array}$ & $\begin{array}{r}1.5 \\
(1.4: 1.5)\end{array}$ & $\begin{array}{l}1.3^{* * *} \\
(1.3 ; 1.4)\end{array}$ & $\begin{array}{r}1.2 \\
(1.2 ; 1.3)\end{array}$ & $\begin{array}{r}1.2^{*} \\
(1.1 ; 1.2)\end{array}$ & $\begin{array}{r}1.4 \\
(1.3 ; 1.4)\end{array}$ & $\begin{array}{r}1.3 \\
(1.2 ; 1.3)\end{array}$ & $\begin{array}{r}1.4 \\
(1.4 ; 1.5)\end{array}$ & $\begin{array}{l}1.4 * * * \\
(1.3 ; 1.4)\end{array}$ \\
\hline $\begin{array}{l}\text { Vegetables } \\
\text { (freq./d) }\end{array}$ & $\begin{array}{r}1.3 \\
(1.2 ; 1.3)\end{array}$ & $\begin{array}{r}1.3^{*} \\
(1.3 ; 1.4)\end{array}$ & $\begin{array}{r}1.4 \\
(1.4 ; 1.5)\end{array}$ & $\begin{array}{r}1.6 * * * \\
(1.5 ; 1.7)\end{array}$ & $\begin{array}{r}1.4 \\
(1.4 ; 1.5)\end{array}$ & $\begin{array}{l}1.6 * * * \\
(1.6 ; 1.7)\end{array}$ & $\begin{array}{r}1.2 \\
(1.1 ; 1.2)\end{array}$ & $\begin{array}{r}1.2 \\
(1.1 ; 1.2)\end{array}$ & $\begin{array}{r}1.9 \\
(1.8: 2.0)\end{array}$ & $\begin{array}{l}1.3^{* * *} \\
(1.2 ; 1.3)\end{array}$ & $\begin{array}{r}1.5 \\
(1.5 ; 1.5)\end{array}$ & $\begin{array}{r}1.5 \\
(1.5 ; 1.5)\end{array}$ \\
\hline $\begin{array}{l}\text { Fish } \\
\text { (freq./week) }\end{array}$ & $\begin{array}{r}1.3 \\
(1.2 ; 1.4)\end{array}$ & $\begin{array}{r}1.2 \\
(1.1 ; 1.3)\end{array}$ & $\begin{array}{r}1.4 \\
(1.3 ; 1.5)\end{array}$ & $\begin{array}{r}1.5 \\
(1.4 ; 1.6)\end{array}$ & $\begin{array}{r}1.5 \\
(1.4 ; 1.6)\end{array}$ & $\begin{array}{r}1.5 \\
(1.4 ; 1.6)\end{array}$ & $\begin{array}{r}1.9 \\
(1.8 ; 2.0)\end{array}$ & $\begin{array}{r}1.9 \\
(1.8 ; 2.0)\end{array}$ & $\begin{array}{r}2.1 \\
(1.9 ; 2.3)\end{array}$ & $\begin{array}{r}2.0 \\
(1.8 ; 2.1)\end{array}$ & $\begin{array}{r}1.6 \\
(1.5 ; 1.6)\end{array}$ & $\begin{array}{r}1.5 \\
(1.5 ; 1.6)\end{array}$ \\
\hline $\begin{array}{l}\text { Whole grain } \\
\text { breads }^{\mathrm{s}} \\
\text { (slices/d) }\end{array}$ & $\begin{array}{r}3.2 \\
(3 \cdot 1 ; 3 \cdot 3)\end{array}$ & $\begin{array}{r}3.2 \\
(3.0 ; 3.3)\end{array}$ & $\begin{array}{r}3.7 \\
(3.5 ; 3.8)\end{array}$ & $\begin{array}{l}3.1^{* * *} \\
(3 \cdot 0: 3 \cdot 3)\end{array}$ & $\begin{array}{r}2.3 \\
(2.2 ; 2.5)\end{array}$ & $\begin{array}{r}2.0 * * * \\
(1.9 ; 2.1)\end{array}$ & $\begin{array}{r}2.1 \\
(2.0 ; 2.1)\end{array}$ & $\begin{array}{r}2.0 \\
(1.9 ; 2.1)\end{array}$ & $\begin{array}{r}4.1 \\
(3.9 ; 4.4)\end{array}$ & $\begin{array}{r}3.9^{-} \\
(3.774 .1)\end{array}$ & $\begin{array}{r}3.2 \\
(3.1 ; 3.2)\end{array}$ & $\begin{array}{r}2.9 * * * \\
(2.8 ; 2.9)\end{array}$ \\
\hline
\end{tabular}

Note: $\quad-p<0.10,{ }^{*} p<0.05,{ }^{* * *} p<0.001$. Differences between survey years 2011 and 2014 using t-tests.

@Weighted according to population size in the five Nordic countries.

\#Excluding fruit juice.

${ }^{s}$ Whole grain bread is defined as wholemeal bread, rye bread and hard bread. 


\subsubsection{Age}

The proportion with an unhealthy diet in the Nordic region was higher in $25-44$-y-olds than in 18-24- and 45-65-y-olds (Table 21).

In Denmark, a higher proportion with an unhealthy diet was found in 25-44- and 45-65-y-olds than in 18-24-y-olds. In Finland, a higher proportion with an unhealthy diet was seen in $25-44^{-}-\mathrm{y}$-olds than in $45-65$-y-olds. In Sweden, the highest proportion with an unhealthy diet was seen in $25-44-y$-olds, and moreover a higher proportion with an unhealthy diet was found in $18-24$ - $y$-olds than in $45-65$-y-olds. In Iceland, a higher proportion with an unhealthy diet was seen in 45-65-y-olds than in 18-24- and 25 44-y-olds. No difference between age groups was found in Norway.

In the Nordic region, the lowest proportions with a high consumption of sugar-rich foods and foods rich in saturated fat were seen in $18-24-y-o l d s$. The intake of fruits and vegetables did not differ between age groups in the Nordic region. In Iceland, 18-24-y-olds had a higher intake of fruits and vegetables than 25-44- and 45-65-y-olds. In Sweden, the lowest intake of fruits and vegetables was seen in $25-44-y$-olds. The intake of fish increased across the age groups in the Nordic region. The intake of fish was higher among the 45-65$y$-olds than in the 18-24-y-olds in all the Nordic countries, except in Denmark.

In the Nordic region between 2011 and 2014, the proportion with an unhealthy diet increased in $25-44-$ and $45-65-y$-olds and decreased in $18-24-y$-olds. The overall dietary quality score showed a decrease in $25-44$ - and $45-65$-y-olds reflecting the increased proportion with an unhealthy diet in these age groups. Data in each of the five Nordic countries showed that the proportion with an unhealthy diet increased from 2011 to 2014 in $25-44$-y-olds in Sweden (from $25.5 \%$ to $35.8 \%$ ), Iceland (from $22.4 \%$ to $31.6 \%$ ) and Norway (from 9.7\% to $15.4 \%$ ) and in $45-65$-y-olds in Denmark (from $19.6 \%$ to $23.5 \%$ ) and Norway (from $5.6 \%$ to $14.1 \%$ ). In the Nordic region, the proportion with a high consumption of sugar-rich foods decreased and the proportion with a high consumption of foods rich in saturated fat increased in 18-24-y-olds between 2011 and 2014. Furthermore, the intake of whole grain bread decreased in 18$24-y$-olds, but the proportion eating at least half of the daily bread intake as whole grain bread increased.

In 25-44-y-olds, the proportions with a high consumption of sugar-rich foods and whole grain bread decreased from 2011 to 2014 while the proportion with a high consumption of foods rich in saturated fat increased. 
In 45-65-y-olds, an increase in the proportion with a high consumption of foods rich in saturated fat and a decrease in the intake of fish and whole grain bread were seen between 2011 and 2014 .

In 25-44-y-olds, the intake of whole grain bread decreased in Denmark, Finland, Sweden and Iceland. For other foods, mixed results of the development from 2011 to 2014 were found in the three age groups in the Nordic countries.

Table 21: Mean and proportion ( $95 \% \mathrm{Cl}$ ) for estimates of dietary intake among adults according to age in the Nordic region. NORMO 2011 and 2014

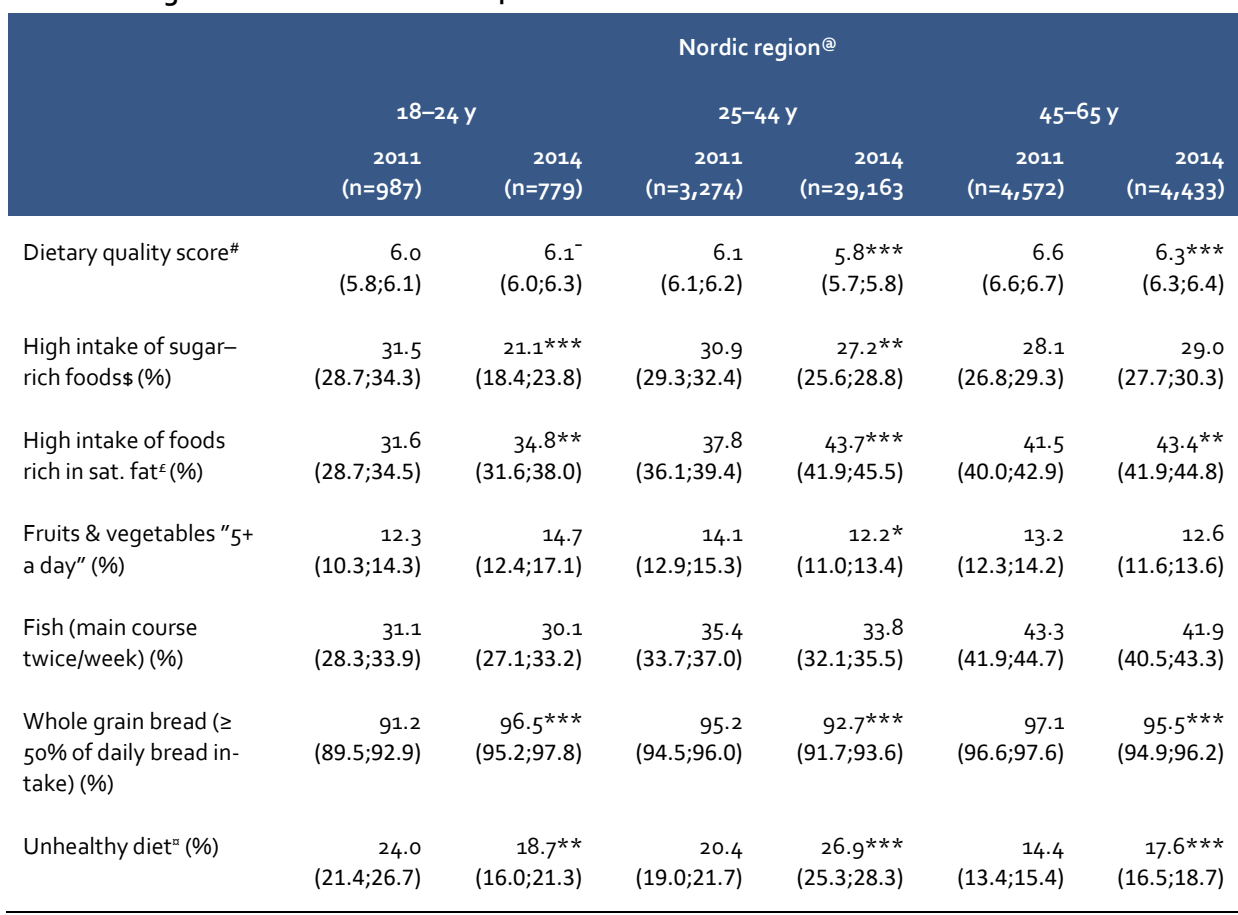

Note: $\quad-p<0.10,{ }^{*} p<0.05,{ }^{* *} p<0.01,{ }^{* * *} p<0.001$. Differences between survey years 2011 and 2014 using ttests and Chi-square tests.

@Weighted according to population size in the five Nordic countries.

\#Overall dietary quality score between o and 12 points based on intakes of fruits and vegetables, whole grain bread, fish as a main course, sugar-rich foods (chocolate/candy, cakes, soft drinks), pommes frites, type of fat used on bread, full fat cheese and sausages.

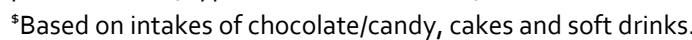

${ }^{\text {f }}$ Based on intake of full fat cheese and type of fat used on bread and for cooking.

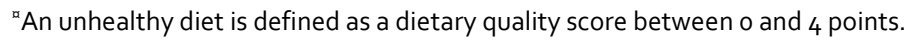


Table 22: Mean $(95 \% \mathrm{Cl})$ intake of selected foods among adults according to age in the Nordic region. NORMO 2011 and 2014

\begin{tabular}{|c|c|c|c|c|c|c|}
\hline & \multicolumn{6}{|c|}{ Nordic region@ } \\
\hline & \multicolumn{2}{|c|}{$18-24 y$} & \multicolumn{2}{|c|}{$25-44 Y$} & \multicolumn{2}{|c|}{$45^{-65 y}$} \\
\hline & $\begin{array}{r}2011 \\
(n=987)\end{array}$ & $\begin{array}{r}2014 \\
(n=779)\end{array}$ & $\begin{array}{r}2011 \\
(n=3,274)\end{array}$ & $\begin{array}{r}2014 \\
(n=29,163)\end{array}$ & $\begin{array}{r}2011 \\
(n=4,572)\end{array}$ & $\begin{array}{r}2014 \\
(n=4,433)\end{array}$ \\
\hline $\begin{array}{l}\text { Fruits \& vegetables } \\
\text { (freq./d) }\end{array}$ & $\begin{array}{r}2.5 \\
(2.4 ; 2.6)\end{array}$ & $\begin{array}{r}2.5 \\
(2.4 ; 2.6)\end{array}$ & $\begin{array}{r}2.6 \\
(2.5 ; 2.6)\end{array}$ & $\begin{array}{r}2.5^{-} \\
(2.5 ; 2.6)\end{array}$ & $\begin{array}{r}2.6 \\
(2.5 ; 2.6)\end{array}$ & $\begin{array}{r}2.5 \\
(2.5 ; 2.6)\end{array}$ \\
\hline Fruits" (freq./d) & $\begin{array}{r}1.2 \\
(1.1 ; 1.2)\end{array}$ & $\begin{array}{r}1.2 \\
(1.1 ; 1.2)\end{array}$ & $\begin{array}{r}1.2 \\
(1.2 ; 1.2)\end{array}$ & $\begin{array}{r}1.1 * * \\
(1.1 ; 1.2)\end{array}$ & $\begin{array}{r}1.3 \\
(1.3 ; 1.3)\end{array}$ & $\begin{array}{r}1.3^{* *} \\
(1.2 ; 1.3)\end{array}$ \\
\hline Vegetables (freq./d) & $\begin{array}{r}1.3 \\
(1.2 ; 1.4)\end{array}$ & $\begin{array}{r}1.4 \\
(1.3 ; 1.4)\end{array}$ & $\begin{array}{r}1.4 \\
(1.3 ; 1.4)\end{array}$ & $\begin{array}{r}1.4 \\
(1.4 ; 1.4)\end{array}$ & $\begin{array}{r}1.3 \\
(1.2 ; 1.3)\end{array}$ & $\begin{array}{r}1.3 \\
(1.3 ; 1.3)\end{array}$ \\
\hline Fish (freq./week) & $\begin{array}{r}1.2 \\
(1.2 ; 1.3)\end{array}$ & $\begin{array}{r}1.3 \\
(1.2 ; 1.4)\end{array}$ & $\begin{array}{r}1.4 \\
(1.4 ; 1.5)\end{array}$ & $\begin{array}{r}1.4 \\
(1.3 ; 1.4)\end{array}$ & $\begin{array}{r}1.7 \\
(1.6 ; 1.8)\end{array}$ & $\begin{array}{r}1.6 * * \\
(1.5 ; 1.6)\end{array}$ \\
\hline $\begin{array}{l}\text { Whole grain breads } \\
\text { (slices/d) }\end{array}$ & $\begin{array}{r}3.2 \\
(3.0 ; 3.4)\end{array}$ & $\begin{array}{r}2.8^{* *} \\
(2.7 ; 3.0)\end{array}$ & $\begin{array}{r}3.5 \\
(3.4 ; 3.6)\end{array}$ & $\begin{array}{r}3.0 * * * \\
(2.9 ; 3.1)\end{array}$ & $\begin{array}{r}4.0 \\
(3.9 ; 4.0)\end{array}$ & $\begin{array}{r}3.7^{* * *} \\
(3.6 ; 3.7)\end{array}$ \\
\hline
\end{tabular}

Note: $\quad-p<0.10,{ }^{*} p<0.05,{ }^{* *} p<0.01,{ }^{* * *} p<0.001$. Differences between survey years 2011 and 2014 using t-tests. @Weighted according to population size in the five Nordic countries.

\#Excluding fruit juice.

${ }^{\text {\$ } W h o l e ~ g r a i n ~ b r e a d ~ i s ~ d e f i n e d ~ a s ~ w h o l e m e a l ~ b r e a d, ~ r y e ~ b r e a d ~ a n d ~ h a r d ~ b r e a d . ~}$

\subsubsection{Education}

In 2014, an inverse social gradient was found in the proportion with an unhealthy diet in the Nordic region, i.e. the proportion with an unhealthy diet decreased with increasing level of education (Table 23). When analysing each of the five Nordic countries, large social differences $(\geq 20 \%)$ in diet (unhealthy diet) were seen in all countries, except in Iceland with a moderate difference. 
In the Nordic region, the proportion with an unhealthy diet was higher among the low and medium educated than among the high educated. The overall dietary quality score was lower among the low and medium educated than among the high educated accordingly. In Denmark, a higher proportion with an unhealthy diet was seen among the medium educated than among the high educated, while in Finland and Sweden, a higher proportion with an unhealthy diet was found among the low and medium educated than among the high educated. In Norway, a higher proportion with an unhealthy diet was found among the low educated than among the medium and high educated. In Iceland, no difference between education groups was found in unhealthy diet.

In the Nordic region, the highest proportion with a high consumption of sugar-rich foods was found among the low educated while the lowest proportion was found among the high educated (Table 23). The intake of fish and fruits and vegetables increased across the education groups in the Nordic region (Table 24). Almost the same pattern was seen for fish in Finland, Sweden and Norway, while no social gradient was seen in Denmark and Iceland. The intake of fruits and vegetables was higher in the higher educated than the low and medium educated in all countries, except Norway. In Norway the high educated had a higher intake of fruits and vegetables than the low educated. The intake of whole grain bread was higher among the low educated than the medium and high educated. 
Table 23: Mean and proportion $(95 \% \mathrm{Cl})$ for estimates of dietary intake among adults according to education in the Nordic region. NORMO 2011 and 2014

\begin{tabular}{|c|c|c|c|c|c|c|}
\hline & \multicolumn{6}{|c|}{ Nordic region@ } \\
\hline & \multicolumn{2}{|c|}{ Low } & \multicolumn{2}{|c|}{ Medium } & \multicolumn{2}{|c|}{ High } \\
\hline & $\begin{array}{r}2011 \\
(n=1,394)\end{array}$ & $\begin{array}{r}2014 \\
(n=1,002)\end{array}$ & $\begin{array}{r}2011 \\
(n=3,833)\end{array}$ & $\begin{array}{r}2014 \\
(n=2,970)\end{array}$ & $\begin{array}{r}2011 \\
(n=3,570)\end{array}$ & $\begin{array}{r}2014 \\
(n=4,161)\end{array}$ \\
\hline Dietary quality score & $\begin{array}{r}6.2 \\
(6.1 ; 6.3)\end{array}$ & $\begin{array}{r}5.8 * * * \\
(5.7 ; 5.9)\end{array}$ & $\begin{array}{r}6.1 \\
(6.0 ; 6.2)\end{array}$ & $\begin{array}{r}6.0^{*} \\
(5.9 ; 6.1)\end{array}$ & $\begin{array}{r}6.6 \\
(6.6 ; 6.7)\end{array}$ & $\begin{array}{r}6.3^{* * *} \\
(6.3 ; 6.4)\end{array}$ \\
\hline $\begin{array}{l}\text { High intake of sugar-rich } \\
\text { foods }(\%)\end{array}$ & $\begin{array}{r}35.3 \\
(32.6 ; 38.1)\end{array}$ & $\begin{array}{r}33.6 \\
(30.2 ; 36.9)\end{array}$ & $\begin{array}{r}30.5 \\
(29.1 ; 31.9)\end{array}$ & $\begin{array}{r}27.2^{* *} \\
(25.6 ; 28.7)\end{array}$ & $\begin{array}{r}25.5 \\
(24.2 ; 26.9)\end{array}$ & $\begin{array}{r}24 \cdot 4^{*} \\
(23.2 ; 25 \cdot 7)\end{array}$ \\
\hline $\begin{array}{l}\text { High intake of foods rich in } \\
\text { sat. fat }{ }^{ \pm}(\%)\end{array}$ & $\begin{array}{r}35.8 \\
(33.0 ; 38.7)\end{array}$ & $\begin{array}{r}44.0 * * * \\
(40.4 ; 47.6)\end{array}$ & $\begin{array}{r}40.2 \\
(38.7 ; 41.8)\end{array}$ & $\begin{array}{r}42.2 \\
(40.4 ; 43.9)\end{array}$ & $\begin{array}{r}37.2 \\
(35.7 ; 38.8)\end{array}$ & $\begin{array}{r}40.2 * \\
(38.8 ; 41.7)\end{array}$ \\
\hline $\begin{array}{l}\text { Fruits \& vegetables " } 5+a \\
\text { day" (\%) }\end{array}$ & $\begin{array}{r}10.0 \\
(8.3 ; 11.8)\end{array}$ & $\begin{array}{r}10.8 \\
(8.6 ; 13.0)\end{array}$ & $\begin{array}{r}11.3 \\
(10.3 ; 12.3)\end{array}$ & $\begin{array}{r}12.0 \\
(10.9 ; 13.2)\end{array}$ & $\begin{array}{r}18.3 \\
(17.1 ; 19.5)\end{array}$ & $\begin{array}{r}14.9^{* * *} \\
(13.9 ; 15.9)\end{array}$ \\
\hline $\begin{array}{l}\text { Fish (main course } \\
\text { twice/week) (\%) }\end{array}$ & $\begin{array}{r}31.7 \\
(28.9 ; 34.4)\end{array}$ & $\begin{array}{r}27 \cdot 3^{*} \\
(24 \cdot 1 ; 30 \cdot 4)\end{array}$ & $\begin{array}{r}33.5 \\
(32.1 ; 35.0)\end{array}$ & $\begin{array}{r}34.2 \\
(32.6 ; 35.9)\end{array}$ & $\begin{array}{r}46.3 \\
(44.7 ; 47.8)\end{array}$ & $\begin{array}{r}43.8^{*} \\
(42.4 ; 45.3)\end{array}$ \\
\hline $\begin{array}{l}\text { Whole grain bread }(\geq 50 \% \\
\text { of daily bread intake) }(\%)\end{array}$ & $\begin{array}{r}95.2 \\
(93.9 ; 96.4)\end{array}$ & $\begin{array}{r}92.8 * \\
(90.9 ; 94.7)\end{array}$ & $\begin{array}{r}94.7 \\
(94.0 ; 95.4)\end{array}$ & $\begin{array}{r}94.6 \\
(93.8 ; 95.4)\end{array}$ & $\begin{array}{r}96.9 \\
(96.3 ; 97.4)\end{array}$ & $\begin{array}{r}95.5^{* *} \\
(94.9 ; 96.1)\end{array}$ \\
\hline Unhealthy diet ${ }^{\mathrm{x}}(\%)$ & $\begin{array}{r}20.6 \\
(18.2 ; 23.1)\end{array}$ & $\begin{array}{r}25.7^{*} \\
(22.5 ; 28.9)\end{array}$ & $\begin{array}{r}21.2 \\
(19.9 ; 22.5)\end{array}$ & $\begin{array}{r}22.9^{-} \\
(21.4 ; 24.4)\end{array}$ & $\begin{array}{r}13.4 \\
(12.3 ; 14.5)\end{array}$ & $\begin{array}{r}17.0 * * * \\
(15.8 ; 18.1)\end{array}$ \\
\hline $\begin{array}{l}\text { Relative difference un- } \\
\text { healthy diet" (\%) }\end{array}$ & - & - & - & - & -54 & -51 \\
\hline
\end{tabular}

Note: $\quad-p<0.10, * p<0.05, * * p<0.01, * * * p<0.001$. Differences between survey years 2011 and 2014 using ttests and Chi-square tests.

@Weighted according to population size in the five Nordic countries.

\#Overall dietary quality score between o and 12 points based on intakes of fruits and vegetables, whole grain bread, fish as a main course, sugar-rich foods (chocolate/candy, cakes, soft drinks), pommes frites, type of fat used on bread, full fat cheese and sausages).

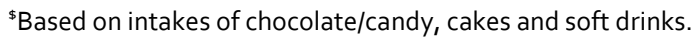

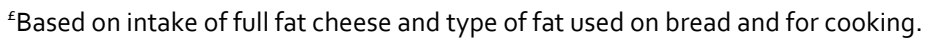

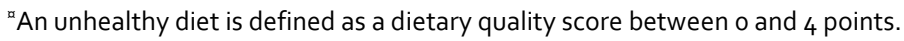

"Relative difference $(\%)=[($ value High education group - value Low education group $) /$

value High education group] ${ }^{*} 100$. 
Table 24: Mean $(95 \% \mathrm{CI})$ intake of selected foods among adults according to education in the Nordic region. NORMO 2011 and 2014

\begin{tabular}{|c|c|c|c|c|c|c|}
\hline & \multicolumn{6}{|c|}{ Nordic region@ } \\
\hline & \multicolumn{2}{|c|}{ Low } & \multicolumn{2}{|c|}{ Medium } & \multicolumn{2}{|c|}{ High } \\
\hline & $\begin{array}{r}2011 \\
(n=1,394)\end{array}$ & $\begin{array}{r}2014 \\
(n=1,002)\end{array}$ & $\begin{array}{r}2011 \\
(n=3,833)\end{array}$ & $\begin{array}{r}2014 \\
(n=2,970)\end{array}$ & $\begin{array}{r}2011 \\
(n=3,570)\end{array}$ & $\begin{array}{r}2014 \\
(n=4,161)\end{array}$ \\
\hline Fruits \& vegetables ${ }^{\#}$ (freq./d) & $\begin{array}{r}2.3 \\
(2.2 ; 2.4)\end{array}$ & $\begin{array}{r}2.3 \\
(2.2 ; 2.4)\end{array}$ & $\begin{array}{r}2.4 \\
(2.4 ; 2.5)\end{array}$ & $\begin{array}{r}2.4 \\
(2.4 ; 2.5)\end{array}$ & $\begin{array}{r}2.9 \\
(2.8 ; 2.9)\end{array}$ & $\begin{array}{r}2.8 * * \\
(2.7 ; 2.8)\end{array}$ \\
\hline Fruits\# (freq./d) & $\begin{array}{r}1.2 \\
(1.2 ; 1.3)\end{array}$ & $\begin{array}{r}1.1 * \\
(1.1 ; 1.2)\end{array}$ & $\begin{array}{r}1.2 \\
(1.1 ; 1.2)\end{array}$ & $\begin{array}{r}1.1 \\
(1.1 ; 1.2)\end{array}$ & $\begin{array}{r}1.4 \\
(1.3 ; 1.4)\end{array}$ & $\begin{array}{r}1.3^{* *} \\
(1.3 ; 1.3)\end{array}$ \\
\hline Vegetables(freq./d) & $\begin{array}{r}1.1 \\
(1.0 ; 1.1)\end{array}$ & $\begin{array}{r}1.1 * \\
(1.1 ; 1.2)\end{array}$ & $\begin{array}{r}1.2 \\
(1.2 ; 1.3)\end{array}$ & $\begin{array}{r}1.3^{*} \\
(1.3 ; 1.3)\end{array}$ & $\begin{array}{r}1.5 \\
(1.5 ; 1.6)\end{array}$ & $\begin{array}{r}1.5 \\
(1.5 ; 1.5)\end{array}$ \\
\hline Fish (freq./week) & $\begin{array}{r}1.4 \\
(1.3 ; 1.4)\end{array}$ & $\begin{array}{r}1.2 * \\
(1.1 ; 1.3)\end{array}$ & $\begin{array}{r}1.4 \\
(1.4 ; 1.5)\end{array}$ & $\begin{array}{r}1.4 \\
(1.3 ; 1.5)\end{array}$ & $\begin{array}{r}1.7 \\
(1.7 ; 1.8)\end{array}$ & $\begin{array}{r}1.6 * \\
(1.6 ; 1.7)\end{array}$ \\
\hline Whole grain bread\$ (slices/d) & $\begin{array}{r}4.0 \\
(3.8 ; 4.2)\end{array}$ & $\begin{array}{r}3.6 * * \\
(3.4 i 3.8)\end{array}$ & $\begin{array}{r}3.7 \\
(3.6 ; 3.7)\end{array}$ & $\begin{array}{l}3.2 * * * \\
(3.1 ; 3 \cdot 3)\end{array}$ & $\begin{array}{r}3.5 \\
(3.5 ; 3.6)\end{array}$ & $\begin{array}{c}3.1 * * * \\
(3.1 ; 3.2)\end{array}$ \\
\hline
\end{tabular}

Note: $\quad * p<0.05, * * p<0.01, * * * p<0.001$. Differences between survey years 2011 and 2014 using t-tests.

@Weighted according to population size in the five Nordic countries.

"Excluding fruit juice.

\$Whole grain bread is defined as wholemeal bread, rye bread and hard bread.

The proportion with an unhealthy diet increased among the low and high educated from 2011 to 2014 in the Nordic region. In accordance with these results, the overall dietary quality score decreased in all education groups. Analyses in each of the five Nordic countries found that the proportion with an unhealthy diet increased between 2011 and 2014 among the low educated in Sweden (from $22.1 \%$ to $36.1 \%$ ) and Norway (from $10.5 \%$ to $24.6 \%$ ) and among the high educated in Denmark (from $15.1 \%$ to $18.6 \%$ ), Iceland (from $16.0 \%$ to $23.2 \%$ ) and Norway (from $5.0 \%$ to $11.7 \%$ ).

A decrease in the proportion with a high consumption of sugar-rich foods was seen among the medium and high educated in the Nordic region from 2011 to 2014. The proportion with a high consumption of foods rich in saturated fat increased among the low and high educated. The intake of fruits and vegetables decreased among the high educated between 2011 and 2014 while the intake of fish decreased among the low and high educated. Intake of whole grain bread decreased in all education groups (Table 24).

Between 2011 and 2014, the intake of fruits decreased among the high educated in Denmark, Island and Norway and increased in Finland, while the intake of vegetables increased in Denmark, Finland and Norway and decreased in Sweden among the high 
educated. For other foods, mixed results of the development from 2011 to 2014 were seen in the three education groups in the Nordic countries.

As the proportion with an unhealthy diet increased among both the low and high educated between 2011 and 2014, the social difference in diet did not change in the Nordic region (Figure 2).

In Denmark and Iceland, the social difference in diet decreased between 2011 and 2014 due to an increase in the proportion with an unhealthy diet among the high educated. In Sweden, the social difference in diet increased because the proportion with an unhealthy diet increased among the low educated. In Finland no change was seen in proportion with an unhealthy diet among the low and high educated and in Norway an increase in proportion with an unhealthy diet was seen in both the low and high educated. Most of the changes in the low and high education group in each of five Nordic countries were not statistically significant.

Figure 2: The development from 2011 to 2014 in the social difference in diet (unhealthy diet) among adults in the Nordic region. NORMO 2011 and 2014

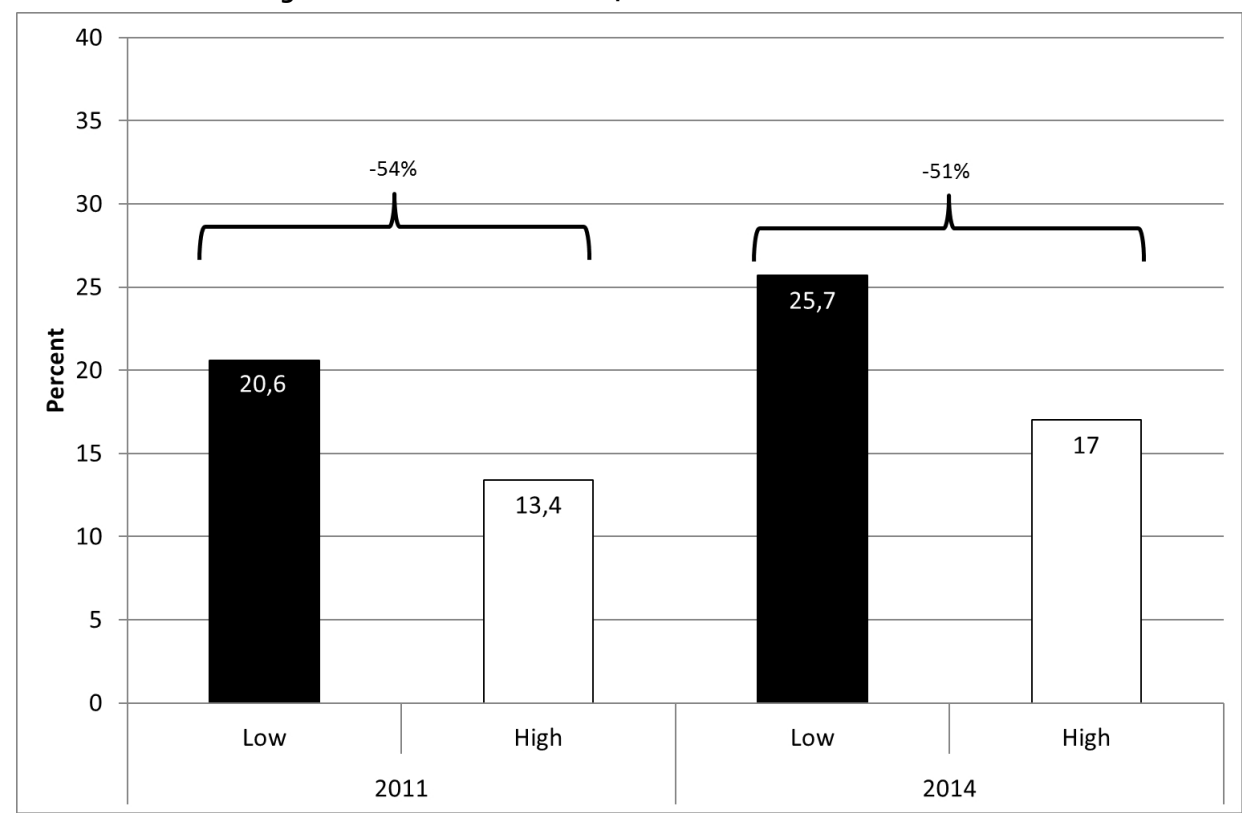




\subsubsection{Children}

Results showed that one in seven children in the Nordic region had an unhealthy diet in 2014. The proportion with an unhealthy diet among children in the five Nordic countries is shown in Table 25. The proportion with an unhealthy diet was higher in Sweden than in the other Nordic countries and higher in Iceland than in Norway.

Table 25: Ranking from lowest to highest proportion with an unhealthy diet among children in the five Nordic countries. NORMO 2014

Table 26 and 27 show changes in the proportions with an unhealthy diet and following specific food based dietary guidelines and mean intake of selected foods among children in the Nordic region. The proportion with an unhealthy diet did not change from 2011 to 2014 among children in the Nordic region, however the proportion with an unhealthy diet decreased among children in Finland.

The lowest proportion with a high consumption of foods rich in saturated fat was found in Denmark. ${ }^{3}$ The lowest proportion with a high consumption of sugar-rich foods was found in Norway (Table 26). 4 The lowest intake of fruits and vegetables was found in Iceland and Norway while the highest intake was found in Denmark and Sweden (Table 27). The lowest intake of fish was seen in Denmark and the highest in Iceland, while the lowest intake of whole grain bread was found in Iceland and Sweden and the highest in Norway.

Almost the same countries was found with the highest or lowest proportions eating " $5+$ a day" (fruits and vegetables), fish twice a week and at least half of their daily bread intake as whole grain bread as when looking at the food intake.

Between 2011 and 2014, the overall dietary quality score and the proportion with an unhealthy diet did not change among children in the Nordic region (Figure 3). Still, some favorable dietary changes occurred from 2011 to 2014 as the proportion with a high consumption of sugar-rich foods decreased among children in the Nordic region (Table 26)

3 High consumption of food rich in saturated fats (fat used on bread and for cooking, full fat cheese). 4 High consumption of sugar-rich foods (chocolate/candy, cakes, sugar sweetened soft drinks). 
and the intake of fruits and vegetables and fish increased (Table 27). In addition, the intake of whole grain bread decreased. Most of the dietary changes were small.

Between 2011 and 2014, the proportion with a high consumption of foods rich in saturated fat decreased among children in Denmark. The intake of fruit and vegetables increased among children in Finland and Sweden and the intake of fish increased among children in Denmark, Finland and Iceland while the intake of whole grain bread decreased among children in Iceland and Norway (Table 26 and 27).

Figure 3: Population level and development in dietary quality among children in the Nordic region. NORMO 2011 and 2014

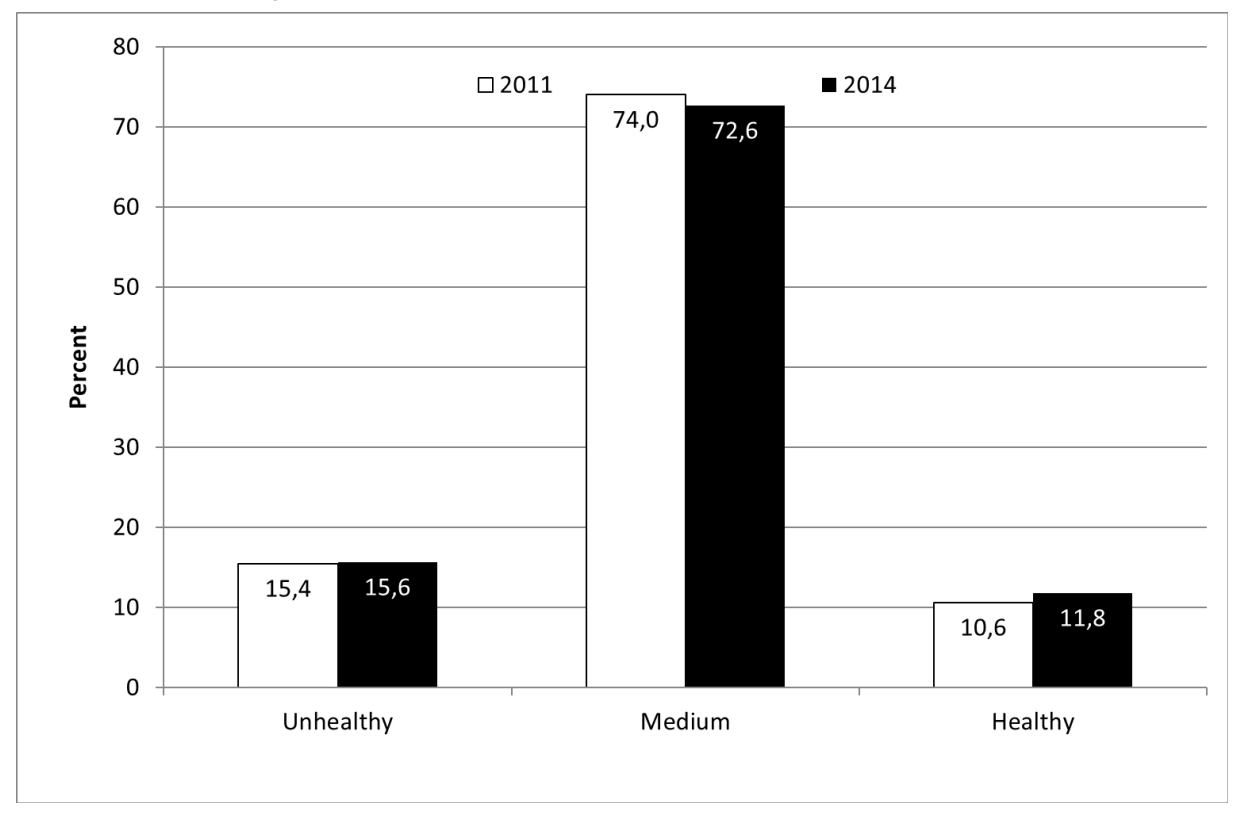


Table 26: Mean and proportion $(95 \% \mathrm{Cl})$ for estimates of dietary intake among children in the Nordic countries. NORMO 2011 and 2014

\begin{tabular}{|c|c|c|c|c|c|c|c|c|c|c|c|c|}
\hline & \multicolumn{2}{|c|}{ Denmark } & \multicolumn{2}{|c|}{ Finland } & \multicolumn{2}{|c|}{ Sweden } & \multicolumn{2}{|c|}{ Iceland } & \multicolumn{2}{|c|}{ Norway } & \multicolumn{2}{|c|}{ Nordic region@ } \\
\hline & $\begin{array}{r}2011 \\
(n=608)\end{array}$ & $\begin{array}{r}2014 \\
(n=592)\end{array}$ & $\begin{array}{r}2011 \\
(n=496)\end{array}$ & $\begin{array}{r}2014 \\
(n=467)\end{array}$ & $\begin{array}{r}2011 \\
(n=492)\end{array}$ & $\begin{array}{r}2014 \\
(n=481)\end{array}$ & $\begin{array}{r}2011 \\
(n=508)\end{array}$ & $\begin{array}{r}2014 \\
(n=493)\end{array}$ & $\begin{array}{r}2011 \\
(n=349)\end{array}$ & $\begin{array}{r}2014 \\
(n=338)\end{array}$ & $\begin{array}{r}2011 \\
(n=2,459)\end{array}$ & $\begin{array}{r}2014 \\
(n=2,377)\end{array}$ \\
\hline Dietary quality score" & $\begin{array}{r}6.4 \\
(6.3 ; 6.5)\end{array}$ & $\begin{array}{r}6.4 \\
(6.3 ; 6.6)\end{array}$ & $\begin{array}{r}6.4 \\
(6.2 ; 6.6)\end{array}$ & $\begin{array}{r}6.6 \\
(6.4 ; 6.7)\end{array}$ & $\begin{array}{r}5.9 \\
(5.7 ; 6.0)\end{array}$ & $\begin{array}{r}5.9 \\
(5.8 ; 6.1)\end{array}$ & $\begin{array}{r}6.5 \\
(6.4 ; 6.7)\end{array}$ & $\begin{array}{r}6.4 \\
(6.2 ; 6.5)\end{array}$ & $\begin{array}{r}6.8 \\
(6.6 ; 7.0)\end{array}$ & $\begin{array}{r}6.8 \\
(6.7 ; 7.0)\end{array}$ & $\begin{array}{r}6.3 \\
(6.2 ; 6.4)\end{array}$ & $\begin{array}{r}6.4 \\
(6.3 ; 6.4)\end{array}$ \\
\hline $\begin{array}{l}\text { High intake of sugar-rich } \\
\text { foods }(\%)\end{array}$ & $\begin{array}{r}26.7 \\
(23.2 ; 30.2)\end{array}$ & $\begin{array}{r}22.1^{-} \\
(18.8 ; 25.4)\end{array}$ & $\begin{array}{r}32.0 \\
(27.9 ; 36.0)\end{array}$ & $\begin{array}{r}26.6^{-} \\
(22.8 ; 30.5)\end{array}$ & $\begin{array}{r}14.5 \\
(11.4 ; 17.6)\end{array}$ & $\begin{array}{r}17.0^{-} \\
(13.7 ; 20.3)\end{array}$ & $\begin{array}{r}32.6 \\
(28.6 ; 36.6)\end{array}$ & $\begin{array}{r}29.2 \\
(25.3 ; 33.2)\end{array}$ & $\begin{array}{r}14.2 \\
(10.5 ; 17.8)\end{array}$ & $\begin{array}{r}9.9 \\
(6.8 ; 13.1)\end{array}$ & $\begin{array}{r}21.0 \\
(19.4 ; 22.6)\end{array}$ & $\begin{array}{r}18.8^{*} \\
(17.3 ; 0.4)\end{array}$ \\
\hline $\begin{array}{l}\text { High intake of foods rich in } \\
\text { sat. fat }{ }^{E}(\%)\end{array}$ & $\begin{array}{r}18.4 \\
(15.4 ; 21.5)\end{array}$ & $\begin{array}{r}13.6^{*} \\
(10.8 ; 16.3)\end{array}$ & $\begin{array}{r}38.7 \\
(34.3 ; 43.0)\end{array}$ & $\begin{array}{r}32.0^{-} \\
(27.9 ; 36.1)\end{array}$ & $\begin{array}{r}40.9 \\
(36.6 ; 45.3)\end{array}$ & $\begin{array}{r}42.5 \\
(38.2 ; 46.9)\end{array}$ & $\begin{array}{r}31.9 \\
(27.8 ; 36.0)\end{array}$ & $\begin{array}{r}37.7^{-} \\
(33.5 ; 41.9)\end{array}$ & $\begin{array}{r}37.7 \\
(32.6 ; 42.8)\end{array}$ & $\begin{array}{r}41.6 \\
(36.5 ; 46.8)\end{array}$ & $\begin{array}{r}34.4 \\
(32.5 ; 36.3)\end{array}$ & $\begin{array}{r}33.7 \\
(31.9 ; 35.6)\end{array}$ \\
\hline $\begin{array}{l}\text { Fruits \& vegetables "5+ a } \\
\text { day" (\%) }\end{array}$ & $\begin{array}{r}18.1 \\
(15.1 ; 21.2)\end{array}$ & $\begin{array}{r}18.2 \\
(15.1 ; 21.3)\end{array}$ & $\begin{array}{r}13.1 \\
(10.1 ; 16.1)\end{array}$ & $\begin{array}{r}12.8 \\
(9.8 ; 15.7)\end{array}$ & $\begin{array}{r}16.6 \\
(13.3 ; 19.9)\end{array}$ & $\begin{array}{r}15.1 \\
(11.9 ; 18.2)\end{array}$ & $\begin{array}{r}14.7 \\
(11.7 ; 17.7)\end{array}$ & $\begin{array}{r}13.0 \\
(10.1 ; 15.9)\end{array}$ & $\begin{array}{r}10.2 \\
(7.1 ; 13.4)\end{array}$ & $\begin{array}{r}13.5 \\
(10.0 ; 17.1)\end{array}$ & $\begin{array}{r}14.9 \\
(13.5 ; 16.3)\end{array}$ & $\begin{array}{r}15.0 \\
(13.5 ; 16.4)\end{array}$ \\
\hline $\begin{array}{l}\text { Fish (main course } \\
\text { twice/week) (\%) }\end{array}$ & $\begin{array}{r}9.2 \\
(6.9 ; 11.5)\end{array}$ & $\begin{array}{r}14.1^{* *} \\
(11.3 ; 16.9)\end{array}$ & $\begin{array}{r}36.0 \\
(31.8 ; 40.2)\end{array}$ & $\begin{array}{r}43.3^{*} \\
(39.0 ; 47.7)\end{array}$ & $\begin{array}{r}39.4 \\
(35.1 ; 43.7)\end{array}$ & $\begin{array}{r}45.8^{*} \\
(41.4 ; 50.1)\end{array}$ & $\begin{array}{r}82.7 \\
(79.4 ; 85.9)\end{array}$ & $\begin{array}{r}86.4^{-} \\
(83.5 ; 89.4)\end{array}$ & $\begin{array}{r}44.4 \\
(39.3 ; 49.6)\end{array}$ & $\begin{array}{r}59.0^{* * *} \\
(53.9 ; 64.1)\end{array}$ & $\begin{array}{r}33.4 \\
(39.1 ; 43.0)\end{array}$ & $\begin{array}{r}41.5^{* * *} \\
(39.6 ; 43.5)\end{array}$ \\
\hline $\begin{array}{l}\text { Whole grain bread ( } \geq 50 \% \\
\text { of daily bread intake) (\%) }\end{array}$ & $\begin{array}{r}95.8 \\
(94.2 ; 97.4)\end{array}$ & $\begin{array}{r}95.8 \\
(94.2 ; 97.4)\end{array}$ & $\begin{array}{r}95.5 \\
(93.6 ; 97.3)\end{array}$ & $\begin{array}{r}92.5^{-} \\
(90.2 ; 94.9)\end{array}$ & $\begin{array}{r}76.4 \\
(72.7 ; 80.2)\end{array}$ & $\begin{array}{r}76.9 \\
(73.1 ; 80.7)\end{array}$ & $\begin{array}{r}98.1 \\
(97.0 ; 99.3)\end{array}$ & $\begin{array}{r}97.9 \\
(96.6 ; 99.2)\end{array}$ & $\begin{array}{r}97.6 \\
(96.0 ; 99.2)\end{array}$ & $\begin{array}{r}92.3^{* *} \\
(89.5 ; 95.1)\end{array}$ & $\begin{array}{r}89.6 \\
(88.4 ; 90.8)\end{array}$ & $\begin{array}{r}87.8 \\
(86.5 ; 89.1)\end{array}$ \\
\hline Unhealthy $\operatorname{diet}^{\mathrm{u}}(\%)$ & $\begin{array}{r}11.9 \\
(9.3 ; 14.4)\end{array}$ & $\begin{array}{r}12.6 \\
(9.9 ; 15.3)\end{array}$ & $\begin{array}{r}18.0 \\
(14.7 ; 21.4)\end{array}$ & $\begin{array}{r}12.8^{*} \\
(9.7 ; 15.8)\end{array}$ & $\begin{array}{r}19.7 \\
(16.2 ; 23.2)\end{array}$ & $\begin{array}{r}22.2 \\
(18.4 ; 25.9)\end{array}$ & $\begin{array}{r}11.8 \\
(9.0 ; 14.6)\end{array}$ & $\begin{array}{r}11.1 \\
(8.3 ; 13.9)\end{array}$ & $\begin{array}{r}10.1 \\
(6.9 ; 13.2)\end{array}$ & $\begin{array}{r}10.1 \\
(6.9 ; 13.3)\end{array}$ & $\begin{array}{r}15.4 \\
(14.0 ; 16.9)\end{array}$ & $\begin{array}{r}15.6 \\
(14.1 ; 17.0)\end{array}$ \\
\hline
\end{tabular}

$\quad \quad \quad-p<0.10,{ }^{*} p<0.05,{ }^{* *} p<0.01,{ }^{* * *} p<0.001$. Differences between survey years 2011 and 2014 using t-tests and Chi-square tests.

@ Weighted according to population size in the five Nordic countries.

\#Overall dietary quality score between o and 12 points based on intakes of fruits and vegetables, whole grain bread, fish as a main course, sugar-rich foods (chocolate/candy, cakes, soft drinks), pommes frites, type of fat used on bread, full fat cheese and sausages.

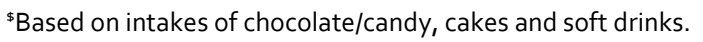

${ }^{\text {E}}$ Based on intake of full fat cheese and type of fat used on bread and for cooking.

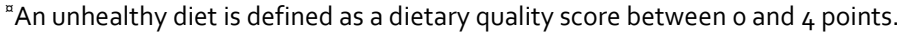


Table 27: Mean $(95 \% \mathrm{Cl})$ intake of selected foods among children in the Nordic countries. NORMO 2011 and 2014

\begin{tabular}{|c|c|c|c|c|c|c|c|c|c|c|c|c|}
\hline & \multicolumn{2}{|c|}{ Denmark } & \multicolumn{2}{|c|}{ Finland } & \multicolumn{2}{|c|}{ Sweden } & \multicolumn{2}{|c|}{ Iceland } & \multicolumn{2}{|c|}{ Norway } & \multicolumn{2}{|c|}{ Nordic region@ } \\
\hline & $\begin{array}{r}2011 \\
(n=608)\end{array}$ & $\begin{array}{r}2014 \\
(n=592)\end{array}$ & $\begin{array}{r}2011 \\
(n=496)\end{array}$ & $\begin{array}{r}2014 \\
(n=467)\end{array}$ & $\begin{array}{r}2011 \\
(n=492)\end{array}$ & $\begin{array}{r}2014 \\
(n=481)\end{array}$ & $\begin{array}{r}2011 \\
(n=508)\end{array}$ & $\begin{array}{r}2014 \\
(n=493)\end{array}$ & $\begin{array}{r}2011 \\
(n=349)\end{array}$ & $\begin{array}{r}2014 \\
\left(n=33^{8}\right)\end{array}$ & $\begin{array}{r}2011 \\
(n=2,459)\end{array}$ & $\begin{array}{r}2014 \\
(n=2,377)\end{array}$ \\
\hline $\begin{array}{l}\text { Fruits \& vegeta- } \\
\text { bles" (freq./day) }\end{array}$ & $\begin{array}{r}3.1 \\
(3.0 ; 3.2)\end{array}$ & $\begin{array}{r}3.1 \\
(3.0 ; 3.2)\end{array}$ & $\begin{array}{r}2.6 \\
(2.5 ; 2.7)\end{array}$ & $\begin{array}{r}2.8^{*} \\
(2.6 ; 2.9)\end{array}$ & $\begin{array}{r}2.8 \\
(2.7 ; 3.0)\end{array}$ & $\begin{array}{r}3.0^{*} \\
(2.9 ; 3.1)\end{array}$ & $\begin{array}{r}2.6 \\
(2.5 ; 2.7)\end{array}$ & $\begin{array}{r}2.6 \\
(2.5 ; 2.7)\end{array}$ & $\begin{array}{r}2.5 \\
(2.3 ; 2.7)\end{array}$ & $\begin{array}{r}2.5 \\
(2.3 ; 2.6)\end{array}$ & $\begin{array}{r}2.8 \\
(2.7 ; 2.8)\end{array}$ & $\begin{array}{r}2.9^{*} \\
(2.8 ; 2.9)\end{array}$ \\
\hline $\begin{array}{l}\text { Fruits\# } \\
\text { (freq./day) }\end{array}$ & $\begin{array}{r}1.7 \\
(1.6 ; 1.8)\end{array}$ & $\begin{array}{r}1.6^{-} \\
(1.5 ; 1.7)\end{array}$ & $\begin{array}{r}1.1 \\
(1.1 ; 1.2)\end{array}$ & $\begin{array}{r}1.2 \\
(1.1 ; 1.3)\end{array}$ & $\begin{array}{r}1.4 \\
(1.4 ; 1.5)\end{array}$ & $\begin{array}{r}1.5 \\
(1.4 ; 1.6)\end{array}$ & $\begin{array}{r}1.4 \\
(1.3 ; 1.5)\end{array}$ & $\begin{array}{r}1.4 \\
(1.3 ; 1.5)\end{array}$ & $\begin{array}{r}1.2 \\
(1.1 ; 1.3)\end{array}$ & $\begin{array}{r}1.3^{*} \\
(1.2 ; 1.4)\end{array}$ & $\begin{array}{r}1.4 \\
(1.3 ; 1.4)\end{array}$ & $\begin{array}{r}1.4 \\
(1.4 ; 1.5)\end{array}$ \\
\hline $\begin{array}{l}\text { Vegetables } \\
\text { (freq./day) }\end{array}$ & $\begin{array}{r}1.4 \\
(1.3 ; 1.5)\end{array}$ & $\begin{array}{r}1.5^{*} \\
(1.4 ; 1.6)\end{array}$ & $\begin{array}{r}1.4 \\
(1.4 ; 1.5)\end{array}$ & $\begin{array}{r}1.6 * \\
(1.5 ; 1.7)\end{array}$ & $\begin{array}{r}1.4 \\
(1.3 ; 1.5)\end{array}$ & $\begin{array}{r}1.5^{* *} \\
(1.5 ; 1.6)\end{array}$ & $\begin{array}{r}1.2 \\
(1.1 ; 1.3)\end{array}$ & $\begin{array}{r}1.2 \\
(1.1 ; 1.2)\end{array}$ & $\begin{array}{r}1.3 \\
(1.2 ; 1.4)\end{array}$ & $\begin{array}{r}1.1^{* *} \\
(1.0 ; 1.2)\end{array}$ & $\begin{array}{r}1.4 \\
(1.4 ; 1.4)\end{array}$ & $\begin{array}{r}1.5^{* *} \\
(1.4 ; 1.5)\end{array}$ \\
\hline $\begin{array}{l}\text { Fish } \\
\text { (freq./week) }\end{array}$ & $\begin{array}{r}0.8 \\
(0.7 ; 0.8)\end{array}$ & $\begin{array}{r}1.0^{*} \\
(0.8 ; 1.1)\end{array}$ & $\begin{array}{r}1.3 \\
(1.2 ; 1.4)\end{array}$ & $\begin{array}{r}1.4^{*} \\
(1.4 ; 1.5)\end{array}$ & $\begin{array}{r}1.4 \\
(1.3 ; 1.5)\end{array}$ & $\begin{array}{r}1.5^{-} \\
(1.4 ; 1.6)\end{array}$ & $\begin{array}{r}2.4 \\
(2.3 ; 2.4)\end{array}$ & $\begin{array}{r}2.6^{*} \\
(2.4 ; 2.8)\end{array}$ & $\begin{array}{r}1.7 \\
(1.4 ; 1.9)\end{array}$ & $\begin{array}{r}1.7 \\
(1.6 ; 1.9)\end{array}$ & $\begin{array}{r}1.3 \\
(1.3 ; 1.4)\end{array}$ & $\begin{array}{r}1.4^{* * *} \\
(1.4 ; 1.5)\end{array}$ \\
\hline $\begin{array}{l}\text { Whole grain } \\
\text { breads }^{\text {s }} \\
\text { (slices/day) }\end{array}$ & $\begin{array}{r}3.6 \\
(3.5 ; 3.8)\end{array}$ & $\begin{array}{r}3.5 \\
(3.4 ; 3.7)\end{array}$ & $\begin{array}{r}3.9 \\
(3.7 ; 4.1)\end{array}$ & $\begin{array}{r}3.7^{-} \\
(3.5 ; 3.9)\end{array}$ & $\begin{array}{r}1.9 \\
(1.7 ; 2.0)\end{array}$ & $\begin{array}{r}1.9 \\
(1.7 ; 2.0)\end{array}$ & $\begin{array}{r}2.2 \\
(2.1 ; 2.3)\end{array}$ & $\begin{array}{r}1.9^{* * *} \\
(1.8 ; 2.0)\end{array}$ & $\begin{array}{r}4.8 \\
(4.5 ; 5.1)\end{array}$ & $\begin{array}{r}4.3^{* *} \\
(4.0 ; 4.6)\end{array}$ & $\begin{array}{r}3.3 \\
(3.2 ; 3.4)\end{array}$ & $\begin{array}{r}3.1^{* *} \\
(3.0 ; 3.2)\end{array}$ \\
\hline
\end{tabular}

$\quad \quad \quad \quad p<0.10,{ }^{*} p<0.05,{ }^{* *} p<0.01,{ }^{* * *} p<0.001$. Differences between survey years 2011 and 2014 using t-tests.

@Weighted according to population size in the five Nordic countries.

\#Excluding fruit juice.

${ }^{\$}$ Whole grain bread is defined as wholemeal bread, rye bread and hard bread. 


\subsubsection{Gender}

The proportion with an unhealthy diet was higher in boys than in girls in the Nordic region (17.5\% vs. 13.7\%). This gender difference was also found in Sweden and Norway.

The intake of fruits and vegetables was higher in girls than in boys in the Nordic region. This gender difference was also seen in Sweden, Iceland and Norway while the opposite was seen in Finland. The intake of whole grain bread was higher in boys than in girls in the Nordic region and in Norway.

Between 2011 and 2014, it was not possible to detect any difference in the proportion with an unhealthy diet in boys and girls in the Nordic region or in each of the five Nordic countries, apart from a decrease in boys in Finland (Table 28 and 30). Although not significant, an upward trend in the proportion with an unhealthy diet was found in girls in Denmark, Finland and Sweden.

Few significant changes were found between 2011 and 2014 when analysing diet according to gender in the Nordic region. Among boys in the Nordic region, the intake of fruits and vegetables increased from 2011 to 2014. Among girls, the intake of fish increased while the intake of whole grain bread decreased

The proportion with a high intake of saturated fat decreased among boys in Denmark and Finland while the intake of fruits and vegetables increased among boys in Finland and Sweden. The proportion with a high intake of saturated fat and a high consumption of sugar-rich foods decreased among girls in Norway. The intake of whole grain bread decreased among boys in Iceland and among girls in Denmark and Iceland. 
Table 28: Mean and proportion $(95 \% \mathrm{Cl}$ ) for estimates of dietary intake among boys in the Nordic countries. NORMO 2011 and 2014

\begin{tabular}{|c|c|c|c|c|c|c|c|c|c|c|c|c|}
\hline & \multicolumn{2}{|c|}{ Denmark } & \multicolumn{2}{|c|}{ Finland } & \multicolumn{2}{|c|}{ Sweden } & \multicolumn{2}{|c|}{ Iceland } & \multicolumn{2}{|c|}{ Norway } & \multicolumn{2}{|c|}{ Nordic region@ } \\
\hline & $\begin{array}{r}2011 \\
(n=318)\end{array}$ & $\begin{array}{r}2014 \\
(n=293)\end{array}$ & $\begin{array}{r}2011 \\
(n=246)\end{array}$ & $\begin{array}{r}2014 \\
(n=225)\end{array}$ & $\begin{array}{r}2011 \\
(n=269)\end{array}$ & $\begin{array}{r}2014 \\
(n=244)\end{array}$ & $\begin{array}{r}2011 \\
(n=240)\end{array}$ & $\begin{array}{r}2014 \\
(n=249)\end{array}$ & $\begin{array}{r}2011 \\
(n=166)\end{array}$ & $\begin{array}{r}2014 \\
(n=164)\end{array}$ & $\begin{array}{r}2011 \\
(n=1,239)\end{array}$ & $\begin{array}{r}2014 \\
(n=1,179)\end{array}$ \\
\hline Dietary quality score ${ }^{\#}$ & $\begin{array}{r}6.3 \\
(6.1 ; 6.5)\end{array}$ & $\begin{array}{r}6.6^{*} \\
(6.4 ; 6.8)\end{array}$ & $\begin{array}{r}6.2 \\
(6.0 ; 6.5)\end{array}$ & $\begin{array}{r}6.6^{*} \\
(6.4 ; 6.9)\end{array}$ & $\begin{array}{r}5.7 \\
(5.5 ; 5.9)\end{array}$ & $\begin{array}{r}5.7 \\
(5.5 ; 5.9)\end{array}$ & $\begin{array}{r}6.6 \\
(6.4 ; 6.8)\end{array}$ & $\begin{array}{r}6.3^{-} \\
(6.1 ; 6.5)\end{array}$ & $\begin{array}{r}6.7 \\
(6.5 ; 7.0)\end{array}$ & $\begin{array}{r}6.5 \\
(6.2 ; 6.8)\end{array}$ & $\begin{array}{r}6.1 \\
(6.0 ; 6.2)\end{array}$ & $\begin{array}{r}6.2 \\
(6.1 ; 6.3)\end{array}$ \\
\hline $\begin{array}{l}\text { High intake of sugar- } \\
\text { rich foodss }(\%)\end{array}$ & $\begin{array}{r}27.1 \\
(22.2 ; 32.0)\end{array}$ & $\begin{array}{r}21.0 \\
(16.4 ; 25.7)\end{array}$ & $\begin{array}{r}33.6 \\
(27.7 ; 39.5)\end{array}$ & $\begin{array}{r}29.8 \\
(24.0 ; 35.5)\end{array}$ & $\begin{array}{r}17.0 \\
(12.5 ; 21.4)\end{array}$ & $\begin{array}{r}20.5 \\
(15.6 ; 25.4)\end{array}$ & $\begin{array}{r}35.6 \\
(29.7 ; 41.6)\end{array}$ & $\begin{array}{r}32.5 \\
(26.7 ; 38.2)\end{array}$ & $\begin{array}{r}12.4 \\
(7.4 ; 17.3)\end{array}$ & $\begin{array}{r}10.4 \\
(5.8 ; 14.9)\end{array}$ & $\begin{array}{r}21.9 \\
(19.6 ; 24.2)\end{array}$ & $\begin{array}{r}20.6^{-} \\
(18.3 ; 22.8)\end{array}$ \\
\hline $\begin{array}{l}\text { High intake of foods } \\
\text { rich in sat. fat }{ }^{ \pm}(\%)\end{array}$ & $\begin{array}{r}16.3 \\
(12.2 ; 20.3)\end{array}$ & $\begin{array}{r}11.0^{*} \\
(7.4 ; 14.6)\end{array}$ & $\begin{array}{r}42.1 \\
(35.8 ; 48.4)\end{array}$ & $\begin{array}{r}28.6 * * \\
(22.8 ; 34.4)\end{array}$ & $\begin{array}{r}44.4 \\
(38.5 ; 50.3)\end{array}$ & $\begin{array}{r}43.8 \\
(37.8 ; 49.9)\end{array}$ & $\begin{array}{r}32.7 \\
(26.8 ; 38.7)\end{array}$ & $\begin{array}{r}38.9 \\
(32.9 ; 44.9)\end{array}$ & $\begin{array}{r}37.5 \\
(30.1 ; 44.9)\end{array}$ & $\begin{array}{r}47.5 \\
(40.1 ; 55.0)\end{array}$ & $\begin{array}{r}35.8 \\
(33.1 ; 38.4)\end{array}$ & $\begin{array}{r}34.3 \\
(31.6 ; 37.0)\end{array}$ \\
\hline $\begin{array}{l}\text { Fruits } \& \text { vegetables " } \\
5+\text { a day" (\%) }\end{array}$ & $\begin{array}{r}12.9 \\
(9.2 ; 16.6)\end{array}$ & $\begin{array}{r}18.9^{*} \\
(14.5 ; 23.4)\end{array}$ & $\begin{array}{r}12.4 \\
(8.3 ; 16.5)\end{array}$ & $\begin{array}{r}14.6 \\
(10.1 ; 19.0)\end{array}$ & $\begin{array}{r}10.5 \\
(6.9 ; 14.2)\end{array}$ & $\begin{array}{r}12.6 \\
(8.5 ; 16.6)\end{array}$ & $\begin{array}{r}14.3 \\
(10.0 ; 18.7)\end{array}$ & $\begin{array}{r}10.2 \\
(6.5 ; 13.9)\end{array}$ & $\begin{array}{r}7.1 \\
(3.3 ; 11.0)\end{array}$ & $\begin{array}{r}5.5 \\
(2.1 ; 8.9)\end{array}$ & $\begin{array}{r}10.8 \\
(9.1 ; 12.5)\end{array}$ & $\begin{array}{r}12.9 \\
(11.0 ; 14.8)\end{array}$ \\
\hline $\begin{array}{l}\text { Fish (main course } \\
\text { twice/week) (\%) }\end{array}$ & $\begin{array}{r}8.7 \\
(5.6 ; 11.8)\end{array}$ & $\begin{array}{r}16.5^{* *} \\
(12.3 ; 20.8)\end{array}$ & $\begin{array}{r}33.8 \\
(27.9 ; 39.7)\end{array}$ & $\begin{array}{r}44 \cdot 7^{*} \\
(38.4 ; 50.9)\end{array}$ & $\begin{array}{r}36.3 \\
(30.5 ; 42.0)\end{array}$ & $\begin{array}{r}41.5 \\
(35.5 ; 47.6)\end{array}$ & $\begin{array}{r}83.8 \\
(79.2 ; 88.4)\end{array}$ & $\begin{array}{r}86.5 \\
(82.4 ; 90.7)\end{array}$ & $\begin{array}{r}42.8 \\
(35.4 ; 50.3)\end{array}$ & $\begin{array}{r}56.6^{*} \\
(49.2 ; 64.1)\end{array}$ & $\begin{array}{r}31.2 \\
(28.7 ; 33.8)\end{array}$ & $\begin{array}{r}40.3^{* * *} \\
(37.5 ; 43.0)\end{array}$ \\
\hline $\begin{array}{l}\text { Whole grain bread ( } \geq \\
50 \% \text { of daily bread in- } \\
\text { take) (\%) }\end{array}$ & $\begin{array}{r}94.9 \\
(92.4 ; 97.3)\end{array}$ & $\begin{array}{r}94.0 \\
(91.3 ; 96.7)\end{array}$ & $\begin{array}{r}94.1 \\
(91.1 ; 97.0)\end{array}$ & $\begin{array}{r}90.4 \\
(86.6 ; 94.3)\end{array}$ & $\begin{array}{r}73.6 \\
(68.3 ; 78.9)\end{array}$ & $\begin{array}{r}77.9 \\
(72.6 ; 83.3)\end{array}$ & $\begin{array}{r}98.6 \\
(97.2 ; 100)\end{array}$ & $\begin{array}{r}97.9 \\
(96.1 ; 99.7)\end{array}$ & $\begin{array}{r}97.3 \\
(94.9 ; 99.8)\end{array}$ & $\begin{array}{r}86.5^{* * *} \\
(81.3 ; 91.7)\end{array}$ & $\begin{array}{r}87.6 \\
(85.5 ; 89.4)\end{array}$ & $\begin{array}{r}86.1 \\
(84.1 ; 88.1)\end{array}$ \\
\hline Unhealthy $\operatorname{diet}^{\mathrm{a}}(\%)$ & $\begin{array}{r}12.3 \\
(8.7 ; 15.9)\end{array}$ & $\begin{array}{r}11.1 \\
(7.5 ; 14.7)\end{array}$ & $\begin{array}{r}22.5 \\
(17.3 ; 27.8)\end{array}$ & $\begin{array}{r}10.6 * * * \\
(6.5 ; 14.6)\end{array}$ & $\begin{array}{r}23.5 \\
(18.5 ; 28.6)\end{array}$ & $\begin{array}{r}27.3 \\
(21.7 ; 32.9)\end{array}$ & $\begin{array}{r}9.4 \\
(5.7 ; 13.1)\end{array}$ & $\begin{array}{r}10.4 \\
(6.6 ; 14.2)\end{array}$ & $\begin{array}{r}9.7 \\
(5.2 ; 14.3)\end{array}$ & $\begin{array}{r}13.8 \\
(8.5 ; 19.1)\end{array}$ & $\begin{array}{r}17.8 \\
(15.7 ; 19.9)\end{array}$ & $\begin{array}{r}17.5 \\
(15.3 ; 19.7)\end{array}$ \\
\hline
\end{tabular}

Note:

- $\mathrm{p}<0.10,{ }^{*} \mathrm{p}<0.05,{ }^{* *} \mathrm{p}<0.01,{ }^{* * *} \mathrm{p}<0.001$. Differences between survey years 2011 and 2014 using t-tests and Chi-square tests.

@Weighted according to population size in the five Nordic countries.

\#Overall dietary quality score between o and 12 points based on intakes of fruits and vegetables, whole grain bread, fish as a main course, sugar-rich foods (choco-

late/candy, cakes, soft drinks), pommes frites, type of fat used on bread, full fat cheese and sausages 2012).

${ }^{s}$ Based on intakes of chocolate/candy, cakes and soft drinks.

${ }^{E}$ Based on intake of full fat cheese and type of fat used on bread and for cooking.

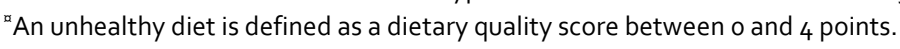


Table 29: Mean ( $95 \% \mathrm{Cl}$ ) intake of selected foods among boys in the Nordic countries. NORMO 2011 and 2014

\begin{tabular}{|c|c|c|c|c|c|c|c|c|c|c|c|c|}
\hline & \multicolumn{2}{|c|}{ Denmark } & \multicolumn{2}{|c|}{ Finland } & \multicolumn{2}{|c|}{ Sweden } & \multicolumn{2}{|c|}{ Iceland } & \multicolumn{2}{|c|}{ Norway } & \multicolumn{2}{|c|}{ Nordic region@ } \\
\hline & $\begin{array}{c}2011 \\
(n=318)\end{array}$ & $\begin{array}{c}2014 \\
(\mathrm{n}=293)\end{array}$ & $\begin{array}{c}2011 \\
(n=246)\end{array}$ & $\begin{array}{c}2014 \\
(n=225)\end{array}$ & $\begin{array}{c}2011 \\
(n=269)\end{array}$ & $\begin{array}{c}2014 \\
(n=244)\end{array}$ & $\begin{array}{c}2011 \\
(n=240)\end{array}$ & $\begin{array}{c}2014 \\
(n=249)\end{array}$ & $\begin{array}{c}2011 \\
(n=166)\end{array}$ & $\begin{array}{c}2014 \\
(n=164)\end{array}$ & $\begin{array}{c}2011 \\
(n=1,239)\end{array}$ & $\begin{array}{c}2014 \\
(n=1,179\end{array}$ \\
\hline $\begin{array}{l}\text { Fruits \& vegeta- } \\
\text { bles" (freq./day) }\end{array}$ & $\begin{array}{c}2.8 \\
(2.7 ; 3.0)\end{array}$ & $\begin{array}{c}3.0 \\
(2.9 ; 3.2)\end{array}$ & $\begin{array}{c}2.4 \\
(2.2 ; 2.6)\end{array}$ & $\begin{array}{c}2.8 * * \\
(2.6 ; 3.0)\end{array}$ & $\begin{array}{c}2.6 \\
(2.4 ; 2.8)\end{array}$ & $\begin{array}{c}2.9^{*} \\
(2.7 ; 3.0)\end{array}$ & $\begin{array}{c}2.5 \\
(2.4 ; 2.7)\end{array}$ & $\begin{array}{c}2.5 \\
(2.3 ; 2.6)\end{array}$ & $\begin{array}{c}2.4 \\
(2.2 ; 2.6)\end{array}$ & $\begin{array}{c}2.2 \\
(2.0 ; 2.4)\end{array}$ & $\begin{array}{c}2.6 \\
(2.5 ; 2.7)\end{array}$ & $\begin{array}{c}2.8 * * \\
(2.7 ; 2.8)\end{array}$ \\
\hline Fruits" (freq./day) & $\begin{array}{c}1.5 \\
(1.4 ; 1.6)\end{array}$ & $\begin{array}{c}1.5 \\
(1.4 ; 1.6)\end{array}$ & $\begin{array}{c}1.0 \\
(0.9 ; 1.1)\end{array}$ & $\begin{array}{c}1.2^{-} \\
(1.1 ; 1.3)\end{array}$ & $\begin{array}{c}1.3 \\
(1.2 ; 1.4)\end{array}$ & $\begin{array}{c}1.4 \\
(1.3 ; 1.5)\end{array}$ & $\begin{array}{c}1.4 \\
(1.3 ; 1.5)\end{array}$ & $\begin{array}{c}1.4 \\
(1.3 ; 1.5)\end{array}$ & $\begin{array}{c}1.2 \\
(1.1 ; 1.3)\end{array}$ & $\begin{array}{c}1.2 \\
(1.1 ; 1.3)\end{array}$ & $\begin{array}{c}1.3 \\
(1.2 ; 1.3)\end{array}$ & $\begin{array}{c}1.3 \\
(1.3 ; 1.4)\end{array}$ \\
\hline $\begin{array}{l}\text { Vegetables } \\
\text { (freq./day) }\end{array}$ & $\begin{array}{c}1.3 \\
(1.2 ; 1.4)\end{array}$ & $\begin{array}{l}1.6 * * * \\
(1.5 ; 1.7)\end{array}$ & $\begin{array}{c}1.4 \\
(1.3 ; 1.5)\end{array}$ & $\begin{array}{c}1.6^{* *} \\
(1.5 ; 1.8)\end{array}$ & $\begin{array}{c}1.3 \\
(1.2 ; 1.4)\end{array}$ & $\begin{array}{c}1.5^{*} \\
(1.4 ; 1.6)\end{array}$ & $\begin{array}{c}1.2 \\
(1.1 ; 1.3)\end{array}$ & $\begin{array}{c}1.1 \\
(1.0 ; 1.2)\end{array}$ & $\begin{array}{c}1.2 \\
(1.1 ; 1.3)\end{array}$ & $\begin{array}{c}1.0^{*} \\
(0.9 ; 1.1)\end{array}$ & $\begin{array}{c}1.3 \\
(1.3 ; 1.3)\end{array}$ & $\begin{array}{c}1.4^{* * *} \\
(1.4 ; 1.5)\end{array}$ \\
\hline Fish (freq./week) & $\begin{array}{c}0.8 \\
(0.6 ; 0.9)\end{array}$ & $\begin{array}{c}1.0^{-} \\
(0.8 ; 1.2)\end{array}$ & $\begin{array}{c}1.3 \\
(1.2 ; 1.4)\end{array}$ & $\begin{array}{c}1.5^{-} \\
(1.4 ; 1.6)\end{array}$ & $\begin{array}{c}1.3 \\
(1.3 ; 1.4)\end{array}$ & $\begin{array}{c}1.4 \\
(1.3 ; 1.5)\end{array}$ & $\begin{array}{c}2.4 \\
(2.3 ; 2.5)\end{array}$ & $\begin{array}{c}2.6 \\
(2.4 ; 2.9)\end{array}$ & $\begin{array}{c}1.6 \\
(1.2 ; 2.0)\end{array}$ & $\begin{array}{c}1.8 \\
(1.5 ; 2.0)\end{array}$ & $\begin{array}{c}1.3 \\
(1.2 ; 1.3)\end{array}$ & $\begin{array}{c}1.4 \\
(1.3 ; 1.4)\end{array}$ \\
\hline 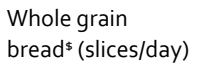 & $\begin{array}{c}3.6 \\
(3.4 ; 3.8)\end{array}$ & $\begin{array}{c}3.7 \\
(3.5 ; 3.9)\end{array}$ & $\begin{array}{c}4.1 \\
(3.8 ; 4.4)\end{array}$ & $\begin{array}{c}3.8 \\
(3.5 ; 4.1)\end{array}$ & $\begin{array}{c}2.1 \\
(1.8 ; 2.3)\end{array}$ & $\begin{array}{c}2.0 \\
(1.8 ; 2.2)\end{array}$ & $\begin{array}{c}2.4 \\
(2.2 ; 2.5)\end{array}$ & $\begin{array}{c}2.0 * * \\
(1.9 ; 2.2)\end{array}$ & $\begin{array}{c}5.2 \\
(4.8 ; 5.6)\end{array}$ & $\begin{array}{c}4.7^{-} \\
(4.2 ; 5.1)\end{array}$ & $\begin{array}{c}3.4 \\
(3.3 ; 3.6)\end{array}$ & $\begin{array}{c}3.3 \\
(3.1 ; 3.4)\end{array}$ \\
\hline
\end{tabular}

$\quad$ Note: $\quad-p<0.10,{ }^{*} p<0.05,{ }^{* *} p<0.01,{ }^{* * *} p<0.001$. Differences between survey years 2011 and 2014 using t-tests.

@Weighted according to population size in the five Nordic countries.

\#Excluding fruit juice.

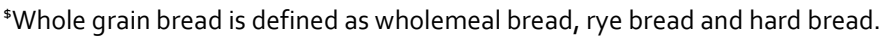


Table 30: Mean and proportion $(95 \% \mathrm{Cl}$ ) forestimates of dietary intake among girls in the Nordic countries. NORMO 2011 and 2014

\begin{tabular}{|c|c|c|c|c|c|c|c|c|c|c|c|c|}
\hline & \multicolumn{2}{|c|}{ Denmark } & \multicolumn{2}{|c|}{ Finland } & \multicolumn{2}{|c|}{ Sweden } & \multicolumn{2}{|c|}{ Iceland } & \multicolumn{2}{|c|}{ Norway } & \multicolumn{2}{|c|}{ Nordic region@ } \\
\hline & $\begin{array}{r}2011 \\
(n=290)\end{array}$ & $\begin{array}{r}2014 \\
(n=299)\end{array}$ & $\begin{array}{r}2011 \\
(n=250)\end{array}$ & $\begin{array}{r}2014 \\
(n=243)\end{array}$ & $\begin{array}{r}2011 \\
(n=223)\end{array}$ & $\begin{array}{r}2014 \\
(n=237)\end{array}$ & $\begin{array}{r}2011 \\
(n=268)\end{array}$ & $\begin{array}{r}2014 \\
(n=244)\end{array}$ & $\begin{array}{r}2011 \\
(n=183)\end{array}$ & $\begin{array}{r}2014 \\
(n=174)\end{array}$ & $\begin{array}{r}2011 \\
(n=1,216)\end{array}$ & $\begin{array}{r}2014 \\
(n=1,198)\end{array}$ \\
\hline Dietary quality score & $\begin{array}{r}6.5 \\
(6.3 ; 6.7)\end{array}$ & $\begin{array}{r}6.3 \\
(6.1 ; 6.4)\end{array}$ & $\begin{array}{r}6.6 \\
(6.4 ; 6.8)\end{array}$ & $\begin{array}{r}6.5 \\
(6.3 ; 6.7)\end{array}$ & $\begin{array}{r}6.1 \\
(5.9 ; 6.3)\end{array}$ & $\begin{array}{r}6.1 \\
(5.9 ; 6.4)\end{array}$ & $\begin{array}{r}6.5 \\
(6.3 ; 6.7)\end{array}$ & $\begin{array}{r}6.4 \\
(6.2 ; 6.7)\end{array}$ & $\begin{array}{r}6.8 \\
(6.6 ; 7.1)\end{array}$ & $\begin{array}{r}7.1^{-} \\
(6.9 ; 7.4)\end{array}$ & $\begin{array}{r}6.5 \\
(6.4 ; 6.6)\end{array}$ & $\begin{array}{r}6.5 \\
(6.4 ; 6.5)\end{array}$ \\
\hline $\begin{array}{l}\text { High intake of sugar- } \\
\text { rich foods }(\%)\end{array}$ & $\begin{array}{r}26.2 \\
(21.2 ; 31.3)\end{array}$ & $\begin{array}{r}23.2 \\
(18.5 ; 28.0)\end{array}$ & $\begin{array}{r}30.0 \\
(24.4 ; 35.7)\end{array}$ & $\begin{array}{r}23.6 \\
(18.5 ; 28.8)\end{array}$ & $\begin{array}{r}12.1 \\
(7.8 ; 16.3)\end{array}$ & $\begin{array}{r}13.4 \\
(9.1 ; 17.7)\end{array}$ & $\begin{array}{r}29 \cdot 3 \\
(23.9 ; 34.8)\end{array}$ & $\begin{array}{r}26.0 \\
(20.6 ; 31.3)\end{array}$ & $\begin{array}{r}15.9 \\
(10.7 ; 21.2)\end{array}$ & $\begin{array}{r}9.5^{*} \\
(5.2 ; 13.8)\end{array}$ & $\begin{array}{r}20.2 \\
(17.9 ; 22.5)\end{array}$ & $\begin{array}{r}17.1 \\
(15.0 ; 19.2)\end{array}$ \\
\hline $\begin{array}{l}\text { High intake of foods } \\
\text { rich in sat. fat }{ }^{ \pm}(\%)\end{array}$ & $\begin{array}{r}20.7 \\
(16.1 ; 25.4)\end{array}$ & $\begin{array}{r}16.2 \\
(12.0 ; 20.3)\end{array}$ & $\begin{array}{r}34.7 \\
(28.7 ; 40.7)\end{array}$ & $\begin{array}{r}35.2 \\
(29.3 ; 41.0)\end{array}$ & $\begin{array}{r}37.4 \\
(31.1 ; 43.8)\end{array}$ & $\begin{array}{r}41.2 \\
(35.0 ; 47.4)\end{array}$ & $\begin{array}{r}31.0 \\
(25.5 ; 36.6)\end{array}$ & $\begin{array}{r}36.5 \\
(30.5 ; 42.4)\end{array}$ & $\begin{array}{r}38.0 \\
(30.9 ; 45.0)\end{array}$ & $\begin{array}{r}35.9^{*} \\
(28.9 ; 42.9)\end{array}$ & $\begin{array}{r}33.0 \\
(30.4 ; 35.7)\end{array}$ & $\begin{array}{r}33.2 \\
(30.5 ; 35.8)\end{array}$ \\
\hline $\begin{array}{l}\text { Fruits \& vegetables } \\
\text { "5+ a day" (\%) }\end{array}$ & $\begin{array}{r}23.6 \\
(18.7 ; 28.5)\end{array}$ & $\begin{array}{r}17 \cdot 3^{-} \\
(13.1 ; 21.6)\end{array}$ & $\begin{array}{r}13.9 \\
(9.7 ; 18.2)\end{array}$ & $\begin{array}{r}11.0 \\
(7.2 ; 14.9)\end{array}$ & $\begin{array}{r}22.8 \\
(17.3 ; 28.2)\end{array}$ & $\begin{array}{r}17.7 \\
(12.9 ; 22.6)\end{array}$ & $\begin{array}{r}15.1 \\
(10.8 ; 19.4)\end{array}$ & $\begin{array}{r}15.8 \\
(11.3 ; 20.2)\end{array}$ & $\begin{array}{r}13 \cdot 3 \\
(8.4 ; 18.2)\end{array}$ & $\begin{array}{r}21.2^{*} \\
(15.3 ; 27.1)\end{array}$ & $\begin{array}{r}18.8 \\
(16.6 ; 21.0)\end{array}$ & $\begin{array}{r}17.0 \\
(14.9 ; 19.1)\end{array}$ \\
\hline $\begin{array}{l}\text { Fish (main course } \\
\text { twice/week) (\%) }\end{array}$ & $\begin{array}{r}9.7 \\
(6.3 ; 13.1)\end{array}$ & $\begin{array}{r}11.6 \\
(8.0 ; 15.2)\end{array}$ & $\begin{array}{r}38.4 \\
(32.5 ; 44.4)\end{array}$ & $\begin{array}{r}42.1 \\
(36.0 ; 48.1)\end{array}$ & $\begin{array}{r}42.6 \\
(36.2 ; 49.0)\end{array}$ & $\begin{array}{r}50.2 \\
(43.9 ; 56.5)\end{array}$ & $\begin{array}{r}81.5 \\
(76.8 ; 86.1)\end{array}$ & $\begin{array}{r}86.3 \\
(82.1 ; 90.5)\end{array}$ & $\begin{array}{r}46.0 \\
(38.8 ; 53.2)\end{array}$ & $\begin{array}{r}61.2 * * \\
(54.2 ; 68.3)\end{array}$ & $\begin{array}{r}35.7 \\
(33.0 ; 38.4)\end{array}$ & $\begin{array}{r}42.7^{* * *} \\
(40.0 ; 45.5)\end{array}$ \\
\hline $\begin{array}{l}\text { Whole grain bread ( } \geq \\
50 \% \text { of daily bread in- } \\
\text { take) }(\%)\end{array}$ & $\begin{array}{r}96.8 \\
(94.8 ; 98.8)\end{array}$ & $\begin{array}{r}97.6 \\
(95.9 ; 99.3)\end{array}$ & $\begin{array}{r}97.1 \\
(95.0 ; 99.2)\end{array}$ & $\begin{array}{r}94.5 \\
(91.6 ; 97.3)\end{array}$ & $\begin{array}{r}79.3 \\
(73.9 ; 84.6)\end{array}$ & $\begin{array}{r}76.0 \\
(70.5 ; 81.4)\end{array}$ & $\begin{array}{r}97.6 \\
(95.8 ; 99.4)\end{array}$ & $\begin{array}{r}97.9 \\
(96.1 ; 99.7)\end{array}$ & $\begin{array}{r}97.8 \\
(95.7 ; 99.9)\end{array}$ & $\begin{array}{r}98.0 \\
(95.9 ; 100)\end{array}$ & $\begin{array}{r}91.5 \\
(90.0 ; 03.1)\end{array}$ & $\begin{array}{r}89.5 \\
(87.8 ; 91.3)\end{array}$ \\
\hline Unhealthy diet ${ }^{\mathrm{n}}(\%)$ & $\begin{array}{r}11.5 \\
(7.8 ; 15.1)\end{array}$ & $\begin{array}{r}14.2 \\
(10.3 ; 18.2)\end{array}$ & $\begin{array}{r}12.9 \\
(8.8 ; 17.0)\end{array}$ & $\begin{array}{r}14.8 \\
(10.3 ; 19.3)\end{array}$ & $\begin{array}{r}15.9 \\
(11.1 ; 20.7)\end{array}$ & $\begin{array}{r}17.0 \\
(12.2 ; 21.7)\end{array}$ & $\begin{array}{r}14.3 \\
(10.1 ; 18.5)\end{array}$ & $\begin{array}{r}11.8 \\
(7.8 ; 19.9)\end{array}$ & $\begin{array}{r}10.3 \\
(5.9 ; 15.9)\end{array}$ & $\begin{array}{r}6.5 \\
(2.9 ; 10.2)\end{array}$ & $\begin{array}{r}13.0 \\
(11.1 ; 14.9)\end{array}$ & $\begin{array}{r}13.7 \\
(11.7 ; 15.6)\end{array}$ \\
\hline
\end{tabular}

$\quad \quad \quad-p<0.10,{ }^{*} p<0.05,{ }^{* *} p<0.01,{ }^{* * *} p<0.001$. Differences between survey years 2011 and 2014 using t-tests and Chi-square tests.

@Weighted according to population size in the five Nordic countries.

\#Overall dietary quality score between o and 12 points based on intakes of fruits and vegetables, whole grain bread, fish as a main course, sugar-rich foods (chocolate/candy, cakes, soft drinks), pommes frites, type of fat used on bread, full fat cheese and sausages.

sBased on intakes of chocolate/candy, cakes and soft drinks.

${ }^{\text {EB}}$ Based on intake of full fat cheese and type of fat used on bread and for cooking.

${ }^{\text {"An }}$ unhealthy diet is defined as a dietary quality score between $\mathrm{o}$ and 4 points. 
Table 31: Mean (95\% Cl) intake of selected foods among girls in the Nordic countries. NORMO 2011 and 2014

\begin{tabular}{|c|c|c|c|c|c|c|c|c|c|c|c|c|}
\hline & \multicolumn{2}{|c|}{ Denmark } & \multicolumn{2}{|c|}{ Finland } & \multicolumn{2}{|c|}{ Sweden } & \multicolumn{2}{|c|}{ Iceland } & \multicolumn{2}{|c|}{ Norway } & \multicolumn{2}{|c|}{ Nordic region@ } \\
\hline & $\begin{array}{r}2011 \\
(n=290)\end{array}$ & $\begin{array}{r}2014 \\
(n=299)\end{array}$ & $\begin{array}{r}2011 \\
(n=250)\end{array}$ & $\begin{array}{r}2014 \\
(n=243)\end{array}$ & $\begin{array}{r}2011 \\
(n=223)\end{array}$ & $\begin{array}{r}2014 \\
(n=237)\end{array}$ & $\begin{array}{r}2011 \\
(n=268)\end{array}$ & $\begin{array}{r}2014 \\
(n=244)\end{array}$ & $\begin{array}{r}2011 \\
(n=183)\end{array}$ & $\begin{array}{r}2014 \\
(n=174)\end{array}$ & $\begin{array}{r}2011 \\
(n=1,216)\end{array}$ & $\begin{array}{r}2014 \\
(n=1,198)\end{array}$ \\
\hline $\begin{array}{l}\text { Fruits \& vegetables\# } \\
\text { (freq./d) }\end{array}$ & $\begin{array}{r}3.4 \\
(3.2 ; 3.5)\end{array}$ & $\begin{array}{r}3.2 \\
(3.0 ; 3.3)\end{array}$ & $\begin{array}{r}2.8 \\
(2.6 ; 3.0)\end{array}$ & $\begin{array}{r}2.7 \\
(2.6 ; 2.9)\end{array}$ & $\begin{array}{r}3.1 \\
(2.9 ; 3.3)\end{array}$ & $\begin{array}{r}3.2 \\
(3.0 ; 3.4)\end{array}$ & $\begin{array}{r}2.7 \\
(2.5 ; 2.9)\end{array}$ & $\begin{array}{r}2.8 \\
(2.6 ; 2.9)\end{array}$ & $\begin{array}{r}2.6 \\
(2.4 ; 2.9)\end{array}$ & $\begin{array}{r}2.7 \\
(2.5 ; 2.9)\end{array}$ & $\begin{array}{r}3.0 \\
(2.9 ; 3.1)\end{array}$ & $\begin{array}{r}3.0 \\
(2.9 ; 3.1)\end{array}$ \\
\hline $\begin{array}{l}\text { Fruits }^{\#} \\
\text { (freq./d) }\end{array}$ & $\begin{array}{r}1.9 \\
(1.7 ; 2.0)\end{array}$ & $\begin{array}{r}1.7 \\
(1.6 ; 1.8)\end{array}$ & $\begin{array}{r}1.3 \\
(1.2 ; 1.4)\end{array}$ & $\begin{array}{r}1.2^{-} \\
(1.1 ; 1.3)\end{array}$ & $\begin{array}{r}1.6 \\
(1.5 ; 1.7)\end{array}$ & $\begin{array}{r}1.6 \\
(1.5 ; 1.7)\end{array}$ & $\begin{array}{r}1.4 \\
(1.3 ; 1.5)\end{array}$ & $\begin{array}{r}1.5 \\
(1.4 ; 1.6)\end{array}$ & $\begin{array}{r}1.2 \\
(1.0 ; 1.3)\end{array}$ & $\begin{array}{r}1.5^{* *} \\
(1.3 ; 1.6)\end{array}$ & $\begin{array}{r}1.5 \\
(1.4 ; 1.5)\end{array}$ & $\begin{array}{r}1.5 \\
(1.5 ; 1.6)\end{array}$ \\
\hline $\begin{array}{l}\text { Vegetables } \\
\text { (freq./d) }\end{array}$ & $\begin{array}{r}1.5 \\
(1.4 ; 1.6)\end{array}$ & $\begin{array}{r}1.5 \\
(1.4 ; 1.6)\end{array}$ & $\begin{array}{r}1.5 \\
(1.4 ; 1.7)\end{array}$ & $\begin{array}{r}1.5 \\
(1.4 ; 1.6)\end{array}$ & $\begin{array}{r}1.5 \\
(1.4 ; 1.6)\end{array}$ & $\begin{array}{r}1.6^{-} \\
(1.5 ; 1.7)\end{array}$ & $\begin{array}{r}1.3 \\
(1.2 ; 1.4)\end{array}$ & $\begin{array}{r}1.3 \\
(1.2 ; 1.4)\end{array}$ & $\begin{array}{r}1.4 \\
(1.3 ; 1.6)\end{array}$ & $\begin{array}{r}1.3^{-} \\
(1.2 ; 1.4)\end{array}$ & $\begin{array}{r}1.5 \\
(1.4 ; 1.5)\end{array}$ & $\begin{array}{r}1.5 \\
(1.4 ; 1.5)\end{array}$ \\
\hline $\begin{array}{l}\text { Fish } \\
\text { (freq./week) }\end{array}$ & $\begin{array}{r}0.8 \\
(0.6 ; 0.9)\end{array}$ & $\begin{array}{r}1.0 \\
(0.7 ; 1.2)\end{array}$ & $\begin{array}{r}1.4 \\
(1.2 ; 1.5)\end{array}$ & $\begin{array}{r}1.4 \\
(1.3 ; 1.5)\end{array}$ & $\begin{array}{r}1.5 \\
(1.3 ; 1.6)\end{array}$ & $\begin{array}{r}1.6^{-} \\
(1.5 ; 1.8)\end{array}$ & $\begin{array}{r}2.3 \\
(2.2 ; 2.4)\end{array}$ & $\begin{array}{r}2.6 \\
(2.3 ; 2.8)\end{array}$ & $\begin{array}{r}1.8 \\
(1.5 ; 2.0)\end{array}$ & $\begin{array}{r}1.7 \\
(1.6 ; 1.9)\end{array}$ & $\begin{array}{r}1.4 \\
(1.3 ; 1.4)\end{array}$ & $\begin{array}{r}1.5^{*} \\
(1.4 ; 1.6)\end{array}$ \\
\hline $\begin{array}{l}\text { Whole grain breads } \\
\text { (slices/d) }\end{array}$ & $\begin{array}{r}3.6 \\
(3.4 ; 3.8)\end{array}$ & $\begin{array}{r}3.3^{*} \\
(3.1 ; 3.5)\end{array}$ & $\begin{array}{r}3.7 \\
(3.5 ; 4.0)\end{array}$ & $\begin{array}{r}3.6 \\
(3.3 ; 3.8)\end{array}$ & $\begin{array}{r}1.7 \\
(1.5 ; 1.9)\end{array}$ & $\begin{array}{r}1.8 \\
(1.6 ; 1.9)\end{array}$ & $\begin{array}{r}2.1 \\
(1.9 ; 2.3)\end{array}$ & $\begin{array}{r}1.8^{*} \\
(1.7 ; 2.0)\end{array}$ & $\begin{array}{r}4.4 \\
(4.1 ; 4.7)\end{array}$ & $\begin{array}{r}4.0 \\
(3.7 ; 4.3)\end{array}$ & $\begin{array}{r}3.2 \\
(3.0 ; 3.3)\end{array}$ & $\begin{array}{r}2.9 * * \\
(2.8 ; 3.0)\end{array}$ \\
\hline
\end{tabular}

$\quad$ Note: $\quad-p<0.10,{ }^{*} p<0.05,{ }^{* *} p<0.01$. Differences between survey years 2011 and 2014 using t-tests.

@Weighted according to population size in the five Nordic countries.

\#Excluding fruit juice.

${ }^{\text {s } W h o l e ~ g r a i n ~ b r e a d ~ i s ~ d e f i n e d ~ a s ~ w h o l e m e a l ~ b r e a d, ~ r y e ~ b r e a d ~ a n d ~ h a r d ~ b r e a d . ~}$ 


\subsubsection{Parental education}

In 2014, the proportion with an unhealthy diet was higher in children with a low or medium educated parent than in children with a high educated parent in the Nordic region (Table 32).

Table 32: Mean and proportion $(95 \% \mathrm{Cl})$ for estimates of dietary intake among children according to parental education in the Nordic region. NORMO 2011 and 2014

\begin{tabular}{|c|c|c|c|c|c|c|}
\hline & \multicolumn{6}{|c|}{ Nordic region@ } \\
\hline & \multicolumn{2}{|c|}{ Low } & \multicolumn{2}{|c|}{ Medium } & \multicolumn{2}{|c|}{ High } \\
\hline & $\begin{array}{r}2011 \\
(n=177)\end{array}$ & $\begin{array}{r}2014 \\
(n=140)\end{array}$ & $\begin{array}{r}2011 \\
(n=942)\end{array}$ & $\begin{array}{r}2014 \\
(n=600)\end{array}$ & $\begin{array}{r}2011 \\
(n=1,336)\end{array}$ & $\begin{array}{r}2014 \\
(n=1,630)\end{array}$ \\
\hline Dietary quality score ${ }^{\#}$ & $\begin{array}{r}6.2 \\
(6.0 ; 6.4)\end{array}$ & $\begin{array}{r}5.8 * \\
(5.5 ; 6.1)\end{array}$ & $\begin{array}{r}6.2 \\
(6.1 ; 6.3)\end{array}$ & $\begin{array}{r}6.1 \\
(6.0 ; 6.3)\end{array}$ & $\begin{array}{r}6.4 \\
(6.3 ; 6.5)\end{array}$ & $\begin{array}{r}6.6 * * \\
(6.5 ; 6.7)\end{array}$ \\
\hline $\begin{array}{l}\text { High intake of sugar- } \\
\text { rich foods }(\%)\end{array}$ & $\begin{array}{r}27.7 \\
(18.8 ; 36.5)\end{array}$ & $\begin{array}{r}24.9^{*} \\
(16.2 ; 33.7)\end{array}$ & $\begin{array}{r}22.3 \\
(19.8 ; 24.9)\end{array}$ & $\begin{array}{r}20.3 \\
(17.2 ; 23.4)\end{array}$ & $\begin{array}{r}19.6 \\
(17.5 ; 21.8)\end{array}$ & $\begin{array}{r}16.9^{*} \\
(15.2 ; 18.7)\end{array}$ \\
\hline $\begin{array}{l}\text { High intake of foods } \\
\text { rich in sat. fat }{ }^{\mp}(\%)\end{array}$ & $\begin{array}{r}26.1 \\
(17.3 ; 35.0)\end{array}$ & $\begin{array}{r}31.6 \\
(22.2 ; 41.0)\end{array}$ & $\begin{array}{r}37.0 \\
(34.0 ; 40.0)\end{array}$ & $\begin{array}{r}35.5 \\
(31.7 ; 39.2)\end{array}$ & $\begin{array}{r}32.7 \\
(30.2 ; 35.2)\end{array}$ & $\begin{array}{r}34.3 \\
(32.1 ; 36.6)\end{array}$ \\
\hline $\begin{array}{l}\text { Fruits \& vegetables" } \\
5+\text { a day" (\%) }\end{array}$ & $\begin{array}{r}15.0 \\
(8.0 ; 22.1)\end{array}$ & $\begin{array}{r}11.0 \\
(4.5 ; 17.4)\end{array}$ & $\begin{array}{r}13.3 \\
(11.2 ; 15.4)\end{array}$ & $\begin{array}{r}12.3 \\
(9.8 ; 14.8)\end{array}$ & $\begin{array}{r}15.3 \\
(13.4 ; 17.2)\end{array}$ & $\begin{array}{r}16.8 \\
(15.0 ; 18.5)\end{array}$ \\
\hline $\begin{array}{l}\text { Fish (main course } \\
\text { twice/week) (\%) }\end{array}$ & $\begin{array}{r}28.0 \\
(19.1 ; 36.9)\end{array}$ & $\begin{array}{r}30.5 \\
(21.2 ; 39.8)\end{array}$ & $\begin{array}{r}30.5 \\
(27.5 ; 33.1)\end{array}$ & $\begin{array}{r}37 \cdot 3^{* * *} \\
(33.8 ; 41.3)\end{array}$ & $\begin{array}{r}37.5 \\
(34.9 ; 40.0)\end{array}$ & $\begin{array}{r}45 \cdot 3^{* * *} \\
(42.9 ; 47.7)\end{array}$ \\
\hline $\begin{array}{l}\text { Whole grain bread ( } \geq \\
50 \% \text { of daily bread in- } \\
\text { take) }(\%)\end{array}$ & $\begin{array}{r}92.9 \\
(87.8 ; 98.0)\end{array}$ & $\begin{array}{r}84.9 \\
(77.3 ; 92.5)\end{array}$ & $\begin{array}{r}88.1 \\
(86.1 ; 90.1)\end{array}$ & $\begin{array}{r}89.8 \\
(87.4 ; 92.2)\end{array}$ & $\begin{array}{r}89.7 \\
(88.1 ; 91.3)\end{array}$ & $\begin{array}{r}88.3 \\
(86.7 ; 89.8)\end{array}$ \\
\hline Unhealthy diet ${ }^{\square}(\%)$ & $\begin{array}{r}12.2 \\
(5.7 ; 18.8)\end{array}$ & $\begin{array}{r}24.4^{*} \\
(15.4 ; 33.4)\end{array}$ & $\begin{array}{r}18.9 \\
(16.5 ; 21.3)\end{array}$ & $\begin{array}{r}19.3 \\
(16.2 ; 22.5)\end{array}$ & $\begin{array}{r}13.6 \\
(11.7 ; 15.4)\end{array}$ & $\begin{array}{r}10.6 * \\
(9.1 ; 12.0)\end{array}$ \\
\hline $\begin{array}{l}\text { Relative difference un- } \\
\text { healthy diet" (\%) }\end{array}$ & - & - & - & - & 10 & -133 \\
\hline
\end{tabular}

Note: $\quad * p<0.05, * * p<0.01, * * * p<0.001$. Differences between survey years 2011 and 2014 using t-tests and Chi-square tests.

@Weighted according to population size in the five Nordic countries.

\#Overall dietary quality score between o and 12 points based on intakes of fruits and vegetables, whole grain bread, fish as a main course, sugar-rich foods (chocolate/candy, cakes, soft drinks), pommes frites, type of fat used on bread, full fat cheese and sausages.

${ }^{\$}$ Based on intakes of chocolate/candy, cakes and soft drinks.

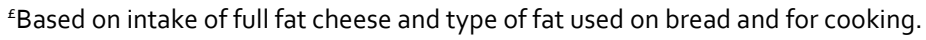

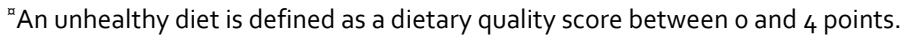

"Relative difference (\%) = [(value High education group - value Low education group)/value High

education group]*100. 
Table 33: Mean $(95 \% \mathrm{Cl})$ intake of selected foods among children according to parental education in the Nordic region. NORMO 2011 and 2014

\begin{tabular}{|c|c|c|c|c|c|c|}
\hline & \multicolumn{6}{|c|}{ Nordic region@ } \\
\hline & \multicolumn{2}{|c|}{ Low } & \multicolumn{2}{|c|}{ Medium } & \multicolumn{2}{|c|}{ High } \\
\hline & $\begin{array}{r}2011 \\
(n=177)\end{array}$ & $\begin{array}{r}2014 \\
(n=140)\end{array}$ & $\begin{array}{r}2011 \\
(n=942)\end{array}$ & $\begin{array}{r}2014 \\
(n=600)\end{array}$ & $\begin{array}{r}2011 \\
(n=1,336)\end{array}$ & $\begin{array}{r}2014 \\
(n=1,630)\end{array}$ \\
\hline $\begin{array}{l}\text { Fruits \& vegetables }{ }^{\#} \\
\text { (freq./d) }\end{array}$ & $\begin{array}{r}2.6 \\
(2.4 ; 2.8)\end{array}$ & $\begin{array}{r}2.7 \\
(2.4 ; 2.9)\end{array}$ & $\begin{array}{r}2.7 \\
(2.6 ; 2.8)\end{array}$ & $\begin{array}{r}2.7 \\
(2.6 ; 2.8)\end{array}$ & $\begin{array}{r}2.9 \\
(2.8 ; 3.0)\end{array}$ & $\begin{array}{l}3.1 * * * \\
(3.0 ; 3.1)\end{array}$ \\
\hline Fruits" (freq./d) & $\begin{array}{r}1.4 \\
(1.3 ; 1.6)\end{array}$ & $\begin{array}{r}1.4 \\
(1.3 ; 1.6)\end{array}$ & $\begin{array}{r}1.4 \\
(1.3 ; 1.4)\end{array}$ & $\begin{array}{r}1.3 \\
(1.3 ; 1.4)\end{array}$ & $\begin{array}{r}1.4 \\
(1.3 ; 1.4)\end{array}$ & $\begin{array}{r}1.5^{* *} \\
(1.4 ; 1.5)\end{array}$ \\
\hline Vegetables (freq./d) & $\begin{array}{r}1.2 \\
(1.1 ; 1.3)\end{array}$ & $\begin{array}{r}1.2 \\
(1.1 ; 1.4)\end{array}$ & $\begin{array}{r}1.3 \\
(1.3 ; 1.4)\end{array}$ & $\begin{array}{r}1.4 \\
(1.3 ; 1.4)\end{array}$ & $\begin{array}{r}1,5 \\
(1.4 ; 1.5)\end{array}$ & $\begin{array}{r}1.6 * * \\
(1.5 ; 1.6)\end{array}$ \\
\hline Fish (freq./week) & $\begin{array}{r}1.2 \\
(1.0 ; 1.4)\end{array}$ & $\begin{array}{r}1.1 \\
(1.0 ; 1.3)\end{array}$ & $\begin{array}{r}1.3 \\
(1.2 ; 1.4)\end{array}$ & $\begin{array}{r}1.4 \\
(1.3 ; 1.5)\end{array}$ & $\begin{array}{r}1.4 \\
(1.3 ; 1.4)\end{array}$ & $\begin{array}{r}1.5^{* * *} \\
(1.5 ; 1.6)\end{array}$ \\
\hline $\begin{array}{l}\text { Whole grain bread\$ } \\
\text { (slices/d) }\end{array}$ & $\begin{array}{r}3.8 \\
(3.4 ; 4.1)\end{array}$ & $\begin{array}{r}2.5^{* * *} \\
(2.2 ; 2.8)\end{array}$ & $\begin{array}{r}3.3 \\
(3.1 ; 3.5)\end{array}$ & $\begin{array}{r}3.2 \\
(; 3.3)\end{array}$ & $\begin{array}{r}3.2 \\
(3.1 ; 3.3)\end{array}$ & $\begin{array}{r}3.2 \\
(3.1 ; 3.3)\end{array}$ \\
\hline
\end{tabular}

Note: $\quad{ }^{* *} \mathrm{p}<0.01,{ }^{* * *} \mathrm{p}<0.001$. Differences between survey years 2011 and 2014 using t-tests.

@Weighted according to population size in the five Nordic countries.

\#Excluding fruit juice.

${ }^{\$}$ Whole grain bread is defined as wholemeal bread, rye bread and hard bread.

The proportion with an unhealthy diet was more than two times higher in children with a low educated parent than in children with a high educated parent suggesting a large inverse social gradient.

Social difference is only reported in the Nordic region because it was not possible to assess social inequality in diet in all the Nordic countries due to few participants in the group of children with a low educated parent. A large social difference in diet (unhealthy diet) was found in the Nordic region. The proportion with an unhealthy diet was higher in children with a low or medium educated parent than in children with a high educated parent in Denmark, Sweden and Iceland. No differences were seen between the three education groups in Finland and Norway.

Only few significant results were seen when analysing the development from 2011 to 2014 in dietary intake according to parental education in the Nordic region. The proportion with an unhealthy diet doubled in children with a low educated parent while a decrease was found in children with a high educated parent (Table 32).

In the Nordic region, the proportion with a high consumption of sugar-rich foods decreased from 2011 to 2014 in children with a low or high educated parent (Table 32). 
Intake of fruits and vegetables and fish increased in children with a high educated parent. Further, intake of whole grain bread decreased in children with a low educated parent. (Table 33). These findings suggest that the development from 2011 to 2014 in dietary intake has mostly been favorable in children with a high educated parent.

Intake of fruits and vegetables increased in children with a medium educated parent in Finland and Iceland, but otherwise mixed results were found in the five Nordic countries.

The social difference in diet increased considerably in the Nordic region between 2011 and 2014 (Figure 4). The increasing social difference was mainly caused by an increase in the proportion with an unhealthy diet in children with a low educated parent.

Figure 4: The development from 2011 to 2014 in the social difference in diet (unhealthy diet) among children in the Nordic region. NORMO 2011 and 2014

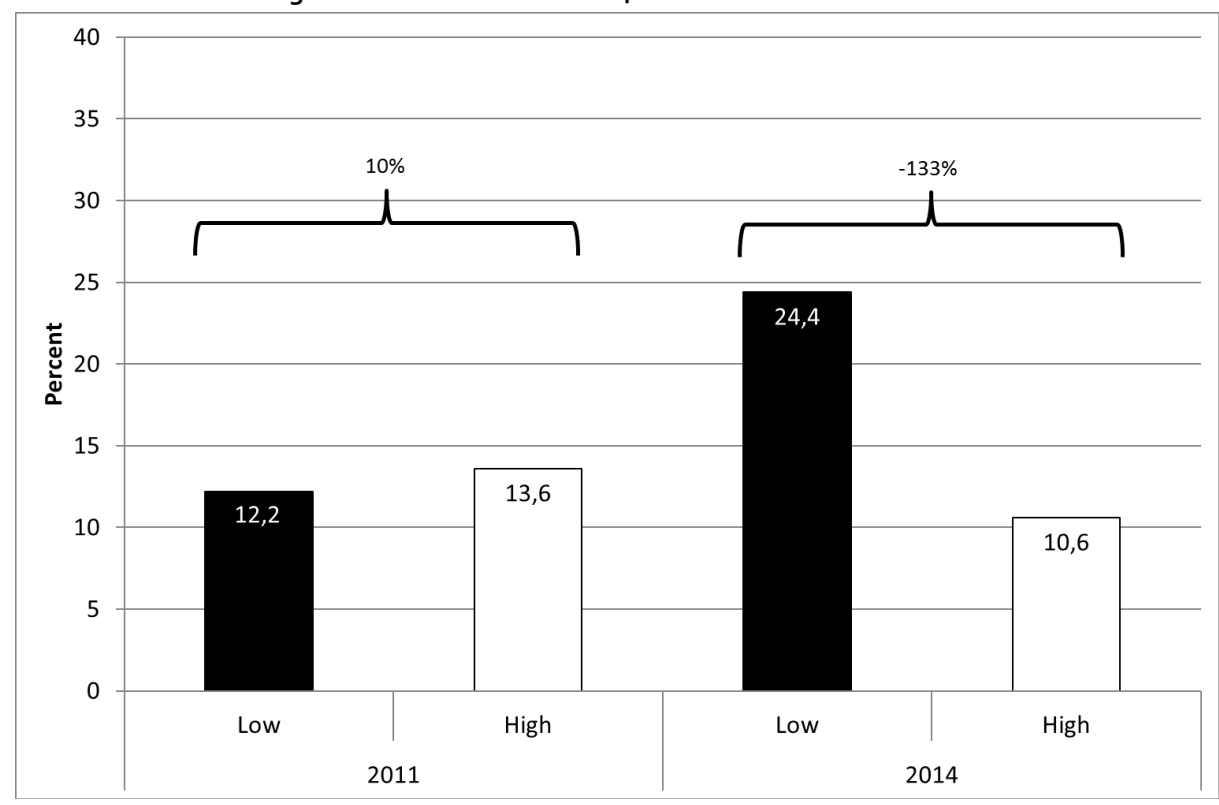

\subsection{Discussion}

\subsubsection{Adults}

Results show an increase in the proportion with an unhealthy diet in the Nordic region from $18.2 \%$ in 2011 to $21.5 \%$ in 2014 . Hence, data indicate that the overall diet has developed unfavorably among adults in the Nordic countries. The increase during the 
three year period is worrying from a public health perspective because it corresponds to more than 500,000 additional adults with an unhealthy diet in the Nordic region (from 3.0 million with an unhealthy diet in 2011 to 3.5 million in 2014). The proportion with an unhealthy diet increased in Sweden, Iceland and Norway.

The proportion with an unhealthy diet increased in both genders, in 25-44- and 4565 -y-olds and among the low and high educated in the Nordic region. However, an encouraging decrease in the proportion with an unhealthy diet was found in $18-24-y$-olds in the Nordic region.

The development in the overall diet quality seems to be heading in an unfavorably direction among adults in the Nordic region even though a decrease in the proportion with a high consumption of sugar-rich foods has occurred and must be considered an improvement.

The most favorable development was seen in Finland because the proportion with an unhealthy diet has come to a halt at a relatively low level. In contrast to Finland, the most unfavorable development was observed in Sweden and Iceland because of a 4-5 percentages point increase in the proportion with an unhealthy diet.

\subsubsection{Comparison with other national estimates from the Nordic countries among adults}

Table 34 shows the recent development according to other national estimates based on data from dietary and health surveys. The proportion fulfilling dietary guidelines regarding fruits and vegetables, fish, whole grain bread/cereals, total fat, saturated fat and added sugar among adults in each of the five Nordic countries are shown. However, some differences in dietary assessment methods, survey periods, participation rates, sample size, age groups and definitions used imply that these estimates are not directly comparable with NORMO data, but still relevant to compare with. Other national estimates cover the time period from 2002 up to 2013 . This time period was selected because Vision 2021 in the Nordic Plan of Action was defined by the use of the proportion fulfilling selected food based dietary guidelines at year 2006 where the Nordic Plan of Action was launched. Only Denmark, Finland and Iceland have data for two time periods and therefore the comparison of changes over time is limited to these countries. 
Table 34: Population levels and the development in meeting food based dietary guidelines and the Nordic Nutrition Recommendations among adults in the Nordic countries according to other national estimates

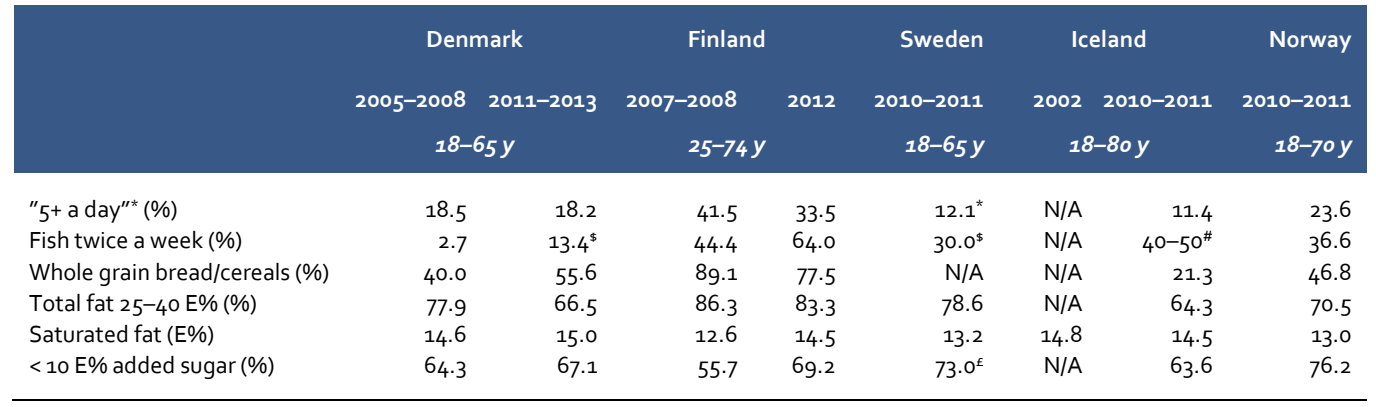

Note: N/A: Not available.

${ }^{*}$ Fruits and vegetables ( $\geq 500 \mathrm{~g} /$ day).

${ }^{\$}$ Fish as a main course ( $\geq 350 \mathrm{~g} /$ week).

${ }^{\#} 50 \%$ eats fish twice a week and $40 \%$ eats $\geq 350 \mathrm{~g}$ fish/week ${ }^{ \pm}$Total sucrose.

Comparison between other national estimates and NORMO data show some similarities, but also some differences. Differences may to a large extent be explained by different sources of data as NORMO data do not cover exactly the same foods/macronutrients as in the other national dietary surveys. For instance, other national estimates cover the proportion eating all kinds of whole grain whereas NORMO only covers the bread intake. Also, NORMO measures slices of bread and this might not be directly comparable to the amounts of total whole grain eaten. Finally, other national estimates might use different definitions of which food to include in the selected dietary variables, thus making it difficult to compare with NORMO data.

Other national estimates for selected foods and macronutrients were only available for Denmark and Finland to describe the development over time (Table 34). Other national estimates for the intake of saturated fat were also available for Iceland.

Other national estimates show no change in the intake of fruits and vegetables among adults in Denmark and a decrease in Finland, while NORMO data also showed no change in Denmark, but an increase in Finland. Other national estimates for Denmark and Finland show an increase in the intake of fish, while NORMO data show a slightly decrease in Denmark and no change in Finland. Finally, other national estimates for whole grain bread/cereals show an increase in Denmark and a decrease in Finland, while NORMO data show a decrease. The development according to other national estimates is therefore only partly in agreement with NORMO data. The most recent survey period for other national estimates of whole grain are 2011-2013 in Denmark and 2012 in Finland. Since the low carb trend has become highly prominent in the Nordic region during recent years, NORMO data might cover the low carb development better 
than other national estimates. It has not been possible to compare all other national estimates with NORMO data.

However, other national estimates are usefull in providing data on the intake of macronutrients like saturated fat and added sugar as these nutrients are not possible to estimate in NORMO. Other national estimates for Denmark and Finland show an increase in the intake of saturated fat and an increase in the proportion with an intake of added sugar below the recommended level while other national estimates for Iceland show no change in the intake of saturated fat. Results from NORMO are in line with other national estimates from Denmark and Finland as an increase in the proportion with a high consumption of foods rich in saturated fat as well as a decrease in the proportion with a high consumption of sugar-rich foods was seen. However, other national estimates from Iceland for the intake of saturated fat are not in accordance with results from NORMO as NORMO data showed an increase in the proportion with a high consumption of foods rich in saturated fat.

The difficulties in obtaining comparable national estimates on foods highlight the importance of collecting data in the Nordic countries in the same period and age groups and using the same assessment method as has been done in NORMO.

In summary, few other national estimates were available to compare with NORMO data. Comparisons between other national estimates and NORMO data show only in part the same development. Also the population levels for diet and nutrients in the Nordic countries differ between other national estimates and NORMO. The discrepancies are probably due to differences in the representativeness of the samples, survey years, assessment methods and definitions of food groups and macronutrients used.

\subsubsection{Evaluation of Goal 2011 and Vision 2021 in the Nordic Plan of Action among adults}

In the Nordic Plan of Action Goals 2011 and Visions 2012 for selected food groups are stated, but not for the overall dietary quality. Goals 2011 in the Nordic Plan of Action was to increase the consumption of fruits and vegetables, fish and whole grain bread/cereals and to decrease the consumption of saturated fat, trans fatty acids and added sugar in the Nordic countries (Appendix C).

The proportion with an unhealthy diet increased among adults in Nordic region and in Sweden, Iceland and Norway while no change was detected in Denmark and Finland. These findings indicate that dietary behaviour is developing unfavorably among adults in the Nordic region. A decrease in the proportion with a high consumption of sugarrich foods was seen. But also a decrease in the intake of fish and whole grain bread and 
an increase in the proportion with a high consumption of foods rich in saturated fat were found. These unfavorable changes were especially attributed to men.

Visions 2021 in the Nordic Plan of Action are to increase the proportion of the population over 10 years eating " $5+$ a day" (at least $500 \mathrm{~g}$ fruit and vegetables/day) as well as eating fish as a main meal twice a week to at least $70 \%$ from the level at year 2006 (Appendix C). Even though these visions may not be evaluated until 2021, one has to be highly optimistic to believe that these visions will be fulfilled taking the current population level and the most recent changes into consideration. In 2014, the proportion eating " $5+$ a day" (fruit and vegetables) was $13 \%$ in the Nordic region. Thus, the current proportion eating " $5+$ a day" should increase fivefold in a seven year period to reach the target level in Vision $2021(\geq 70 \%)$.

In 2014, the proportion eating fish twice a week was $42 \%$ in the Nordic region and the current proportion should increase with approximately $60 \%$ in a seven year period to reach the target population level in Vision 2021. None of the Nordic countries are close to meet the target level of eating " $5+$ a day" or fish as main meal twice a week. However, Iceland is closest to fulfilling the vision 2021 for fish as $64 \%$ of adults in Iceland already eats fish as a main meal twice a week. Denmark is the country with the longest way to go to fulfill the vision on eating enough fish.

Vision 2021 regarding whole grain is to increase the proportion eating at least half of bread intake as whole grain bread/cereals to at least $70 \%$. NORMO only measures the consumption of bread. The vision is fulfilled in all the Nordic countries and $95 \%$ of adults in the Nordic region eat at least half of their daily bread intake as whole grain bread. However, the development from 2011 to 2014 shows a slight downward trend in the proportion eating at least half of their daily bread intake as whole grain bread.

Vision 2021 about fat is that the average dietary intake of saturated plus trans fatty acids should not exceed $10 \%$ of energy and at least $70 \%$ of the population should meet the Nordic nutrition recommendations on fat (E\% from fat between 25 and 40 ). Vision 2021 regarding added sugar is that $80 \%$ or more meets the Nordic nutrition recommendations on added sugar (<10 E\%). As NORMO data do not provide data on macronutrients and energy, neither of these visions may be directly evaluated through NORMO data. Other national estimates show an increase in the proportion with a sugar intake below the recommended level and an increase in the saturated fat intake. NORMO data are in line with these findings. 


\subsubsection{Social inequality}

The social difference in diet (unhealthy diet) did not change from 2011 to 2014 in the Nordic region, but still a large difference between the low and the high education group was seen.

Goal 2011 looks apparently to be fulfilled among adults in the Nordic region as social inequality has not deepened further. Mixed results were found regarding the social difference in diet in the five Nordic countries. Social inequality in diet decreased in Denmark and Iceland between 2011 and 2014 whereas an increase was found in Sweden. No change was found in Finland and Norway.

Goal 2011 was fulfilled among adults in the Nordic region, however not favorably from a public health point of view because the proportion with an unhealthy diet increased in both the low and high education group. This means that health behaviour has deteriorated in both groups. It is therefore important to improve health behaviour in all social groups when the aim is to lower social inequality in health.

Vision 2021 states that social inequality in diet should decrease and that the social difference in diet should be at most $20 \%$. In 2014 , the social difference was $51 \%$ in the Nordic region which indicates a highly inverse social gradient in diet among adults in the Nordic region.

The social difference was large in all the Nordic countries, except in Iceland with a moderate difference. Vision 2021 is not fulfilled and data suggest that the vision may be difficult to fulfill taking the current population level and the development into consideration. Vision 2021 also needs to be evaluated with the ambition of achieving better health status in all social groups and not just decrease the social difference in diet.

\subsubsection{Children}

Results show that the proportion with an unhealthy diet did not change among children in the Nordic region between 2011 and 2014. This was observed in all the Nordic countries, except in Finland with a decrease in the proportion with an unhealthy diet. No change in the proportion with an unhealthy diet was also found from 2011 to 2014 in boys and girls in the Nordic region.

Social inequality in diet increased among children in the Nordic region between 2011 and 2014. This was caused by a large discouraging increase in the proportion with an unhealthy diet in children with a low educated parent and a decrease in children with a high educated parent. Thus, large social differences in diet persist among children in the Nordic region. 


\subsubsection{Comparison with other national estimates from the Nordic countries among children}

Table 35 shows the recent development according to other national estimates based on data from dietary and health surveys. Other national estimates of the proportion fulfilling dietary guidelines regarding fruits and vegetables, fish, whole grain bread/cereals, total fat, saturated fat and added sugar among children in each of the five Nordic countries are shown. Other national estimates are usefull in providing data on intake of macronutrients like saturated fat and added sugar as these nutrients are not covered by NORMO. Other national estimates may differ from NORMO data in terms of representativeness, dietary assessment methods, measures of foods, ${ }^{5}$ survey periods, participation rates, sample size, age groups and definitions used, but are still useful for comparison.

The comparison between other national estimates and NORMO data show some similarities, but also some differences. Differences are described in the section on adults and may to a large extent be explained by different data sources as NORMO data do not cover exactly the same foods/macronutrients as in the other national dietary surveys.

Other national estimates for dietary intake were only available for children in Denmark, Sweden and Iceland. These estimates indicate a downward trend in the intake of saturated fat and added sugar. These findings are in agreement with the non-significant decrease in the proportion with a high consumption of sugar-rich foods seen in NORMO while the decrease in the intake of saturated fat is not in line with NORMO data, where no change was seen. Other national estimates show an increase in fruit and vegetables and fish among children in Denmark, Sweden and Iceland. These findings are in agreement with the increases in fruits and vegetables and fish seen in NORMO. Too few other national estimates were available for the intake of whole grain bread/cereals to enable comparison between data.

In summary, the recent development for children's dietary intake in some of the Nordic countries according to other national estimates is in agreement with NORMO data. However, the population levels for selected foods and macronutrients in the Nordic countries differ between other national estimates and NORMO data probably due to differences in the representativeness of the sample, survey years, and assessment methods and definitions of food groups and macronutrients used.

\footnotetext{
5 Other national estimates for Finland regarding the intake of fruit and vegetables and fish in children are not comparable with NORMO data because other national estimates only cover fruit and vegetables eaten at schools and fish eaten at least once a week.
} 
Table 35: Population levels and the development in meeting food based dietary guidelines and the Nordic Nutrition Recommendations among children in the Nordic countries according to other national estimates

\begin{tabular}{|c|c|c|c|c|c|c|c|c|}
\hline & \multicolumn{2}{|c|}{ Denmark } & Finland & \multicolumn{2}{|c|}{ Sweden } & \multicolumn{2}{|c|}{ Iceland } & \multirow{2}{*}{$\begin{array}{r}\text { Norway } \\
2000 \\
9 y\end{array}$} \\
\hline & $\begin{array}{r}2005-2008 \\
7^{-1}\end{array}$ & $\begin{array}{l}2011-2013 \\
y\end{array}$ & $\begin{array}{r}2007-2008 \\
11 y\end{array}$ & $\begin{array}{r}2003 \\
11-12 y\end{array}$ & $\begin{array}{r}2014 \\
11 y\end{array}$ & $\begin{array}{r}2001 \\
6 y\end{array}$ & 2011 & \\
\hline "5+ a day" (\%) & 17.6 & 19.9 & $66^{+}$ & $0.8^{*}$ & $1.1^{*}$ & 0 & 6 & 2.1 \\
\hline Fish twice a week (\%) & 0.6 & $2.6^{5}$ & $25^{++}$ & $11.4^{\mathrm{s}}$ & $12.1^{\mathrm{s}}$ & 11 & 13 & 17 \\
\hline Whole grain bread/cereals (\%) & 61.0 & 72.8 & $40^{+++}$ & N/A & N/A & N/A & N/A & 27.6 \\
\hline Total fat 25-40 E\% (\%) & 88.1 & 88.8 & N/A & 89.5 & 98.7 & 87.0 & 87.0 & 85.1 \\
\hline Saturated fat (E\%) & 14.0 & 13.9 & $\mathrm{~N} / \mathrm{A}$ & 14.2 & 13.1 & 14.7 & 13.3 & 7.7 \\
\hline$<10$ E\% added sugar (\%) & 36.0 & 49.0 & N/A & $33.4^{\mathrm{E}}$ & $67.0^{ \pm}$ & 26 & 43 & 12.7 \\
\hline
\end{tabular}

Note: N/A: Not available.

+Fresh vegetables daily at school lunch.

*Fruits and vegetables ( $\geq 500 \mathrm{~g} /$ day).

++ Fish at least once a week.

$\$$ Fish as a main course ( $\geq 350 \mathrm{~g} / \mathrm{week}$ ).

+++ Dark bread daily.

€Total sucrose.

\subsubsection{Evaluation of Goal 2011 and Vision 2021 in the Nordic Plan of Action among children}

The goals and visions for dietary intake are the same for children as for adults (Appendix C).

The proportion with an unhealthy diet did not change in Nordic region and in Denmark, Sweden, Iceland and Norway, but a decrease was seen in Finland. The goal regarding fruits and vegetables has been fulfilled in the Nordic region as the intake of fruit and vegetables increased slightlyamong children. The goal on an increase in the intake of whole grain bread/cereals has not been fulfilled. The goal of an increase in the intake of fish has been reached in the Nordic region and in Denmark, Finland and Iceland. Iceland was the only Nordic country with an intake of fish above the target population level in Vision 2021. Other dietary indicators did not change or show mixed results.

The visions will not be evaluated until 2021, but it is doubtful that the visions will be fulfilled taking the current population level and the most recent changes into consideration. In 2014, the proportion eating " $5+$ a day" (fruit and vegetables) was $15 \%$ in the Nordic region (Table 26). Thus, the current proportion eating " $5+$ a day" should increase almost five times in a seven year period to reach the target population level in Vision $2021(\geq 70 \%)$. 
In 2014, the proportion eating fish twice a week was $42 \%$ in the Nordic region and the current proportion should increase significantly to reach the target population level in Vision 2021 ( $\geq 70 \%)$. Only Iceland meets the target population level of eating fish as $86 \%$ of all children eat fish as a main meal twice a week. Denmark is the country with the longest way to go to fulfill the vision on eating enough fish.

Vision 2021 regarding whole grain is fulfilled because $88 \%$ of children in the Nordic region eat at least half of their daily bread intake as whole grain bread. Vision 2021 regarding fat and sugar may not be evaluated with NORMO data because NORMO do not provide data on macronutrients and energy intake. Other national estimates show an increase in the proportion fulfilling the recommendations of eating less than $10 \%$ of the energy deriving from added sugar. This corresponds well with the finding of a decreased proportion with a high consumption of sugar-rich foods in NORMO between 2011 and 2014.

Other national estimates show an decrease in the percentage of energy deriving from saturated fat. NORMO data show in line with this a small non-significant decrease in the proportion with a high consumption of foods rich in saturated fat.

\subsubsection{Social inequality}

The social difference in diet (unhealthy diet) increased from 2011 to 2014 in the Nordic region. While the social difference in diet was small in 2011, it increased to $133 \%$ in 2014. This was mainly due to a large increase in the proportion with an unhealthy diet in children with a low educated parent. Thus, results indicate that social inequality in diet is still highly significant among children in the Nordic region. Goal 2011 is therefore not fulfilled as social inequality has deepened.

Vision 2021 states that the social difference in diet should decrease and be at most $20 \%$. The large increase in the social difference in diet suggests that it may be difficult to fulfill Vision 2021. Vision 2021 needs to be evaluated with the ambition of achieving better health status in all social groups and not just decrease the social difference in diet.

It should also be considered to expand the low education group in future reporting of NORMO data because the group of children with a low educated parent was small in 2011 and 2014 (6-7\%). Expanding the low education group will make it possible to assess social inequality in diet in all the Nordic countries and not just the Nordic region. 


\section{Physical activity and sedentary behaviour}

Physical activity reduces the risk of all-cause mortality and non-communicable diseases such as cardiovascular diseases, obesity, type 2 diabetes and certain type of cancers (Physical Activity Guidelines Advisory Committee 2008, NNR 2012). Physical inactivity has been identified as one of the leading risk factors for major non-communicable diseases and mortality (WHO 2009, Lee et al. 2012). Research suggests that sedentary behaviour may also be a risk factor, independent of physical activity, for adverse health outcomes among adults (Thorp et al. 2011, Stamatakis et al. 2011, Dunstan et al. 2012). Too much sitting is related to cardiovascular disease risk, type 2 diabetes, weight gain and premature mortality.

The population levels and the development from 2011 to 2014 of physical activity and sedentary behaviour in the Nordic countries are presented in this chapter. Time spent on moderate and vigorous intensity physical activity (MVPA) during leisure-time was used to report data on physical activity and time spent on recreational TV time and computer time (sedentary recreational screen time) was used to report data on sedentary behaviour. ${ }^{6}$ Physically inactive is defined as a failure to meet the physical activity recommendations.

The proportions of inactive and with high recreational screen time have been chosen as key variables for physical activity and sedentary behaviour, respectively. The main focus in the Nordic Plan of Action is on physical inactivity (Nordic Council of Ministers, 2006). Classifications of physical activity as inactive, highly inactive (subgroup of inactive), active and highly active (subgroup of active) are shown in Table 5 in the Methods section. Results on the proportion of physically active is tabulated, but will not be commented as physical inactivity and physical activity are two sides of the same coin in this chapter. However, the population levels and the development in subgroups of inactive and active, i.e. the proportions of highly inactive and highly active, will be commented on in the Results and Discussion section. High recreational screen time was defined as more than four hours per day of sedentary TV and/or computer time during leisure.

${ }^{6}$ Sedentary recreational screen time will be referred as just recreational screen time to keep the terms short. 
Data on the population levels and the development of physical activity and sedentary behaviour according to age and education in each of the five Nordic countries are commented in this chapter, but data are not tabulated and only significant findings of the development from 2011 to 2014 will be shown in the text.

The development from 2011 to 2014 for the level of physical at work (adults) and during leisure time (adults and children) are shown in Table 102-103 and 105-106 in appendix $D$, but data will not be commented and were not analysed statistically. These questions have been used in numerous large-scale population surveys in the Nordic countries (Grimby et al. 2015), but the reference period in NORMO is last 7 days in contrast to last year in most of the other surveys.

When comparing physical activity and sedentary behaviour between the Nordic countries, only countries where it is possible to identify one or more countries with the highest and/or lowest proportions of inactive and with high recreational screen time will be commented in the key findings below.

\subsection{Key findings}

\subsubsection{Adults}

\section{Physical activity}

- In 2014, one in three adults were physically inactive in the Nordic region. The proportion of inactive did not differ between genders. The lowest proportion of inactive was found in Finland and Sweden and the highest in Norway.

- In the Nordic region, the proportion of inactive did not change among adults between 2011 and 2014. No change was also found when gender and education were analysed. The proportion of inactive increased only in 18-24-y-olds.

- In the Nordic region, the proportions of highly inactive increased from $10.6 \%$ to $12.4 \%$ between 2011 and 2014 . The proportion of highly inactive increased in women and $18-24-y$-olds and among the medium and high educated.

- In the Nordic region, the proportions of highly active increased from from $13.2 \%$ to $14.4 \%$ between 2011 and 2014 . The proportion of highly active increased in men and among the high educated.

- In the Nordic region, the social difference in physical activity (inactive) was large in 2014 and did not change between 2011 and 2014. 


\section{Sedentary behaviour}

- In 2014, three in ten adults spent more than four hours daily on recreational screen time in the Nordic region. The proportion with high recreational screen time was higher in men than in women ( $32.7 \%$ vs. $27.3 \%$ ). The lowest proportion with high recreational screen time was observed in Sweden and Iceland.

- In the Nordic region, the proportion with high recreational screen time increased slightly from $28.6 \%$ in 2011 to $30.0 \%$ in 2014 among all adults, however no significant change was found in men or women. The proportion with high recreational screen time increased in 45-65-y-olds and among the low educated.

- In the Nordic region, the social difference in sedentary behaviour (high recreational screen time) was large in 2014 and increased between 2011 and 2014 .

\subsubsection{Children}

\section{Physical activity}

- In 2014, six in ten children were physically inactive in the Nordic region. The proportion of inactive was higher in girls than in boys (64.2\% vs. $54.0 \%)$. The lowest proportion of inactive was observed in Finland and the highest in Sweden.

- In the Nordic region, the proportion of inactive did not change among children between 2011 and 2014. No change was also found when gender and (parental) education were analysed.

- In girls, the proportion of highly inactive decreased from 2011 to 2014 .

- In the Nordic region, the social difference in physical activity (inactive) was small in 2014 and did not change between 2011 and 2014.

\section{Sedentary behaviour}

- In 2014, more than one in seven children spent more than four hours daily on recreational screen time in the Nordic region. The proportion with high recreational screen time was higher in boys than in girls (18.2\% vs. $12.8 \%)$. The lowest proportion with high recreational screen time was observed in Iceland.

- In the Nordic region, the proportion with high recreational screen time did not change between 2011 and 2014. No change was also found in boys and when (parental) education was analysed. However, the proportion with high recreational screen time decreased in girls from 2011 to 2014 . 
- In the Nordic region, the social difference in sedentary behaviour (high recreational screen time) was small in 2014 and decreased considerably between 2011 and 2014.

\subsubsection{Conclusions}

Results indicate that the lifestyle has become more inactive and sedentary among adults in the Nordic region between 2011 and 2014, although an increased polarisation in the physical activity pattern and only a slight change in sedentary behaviour were found. This is however not evident among children in the Nordic region where the level of physical activity and sedentary behaviour did not change. Goal 2011 in the Nordic Plan of Action has therefore been fulfilled among children, but not among adults. The social difference in physical activity (inactive) was still large among adults and still small among children in the Nordic region. Data also indicate that social inequality in physical activity has not deepened further among adults and children.

\subsection{Results}

\subsubsection{Adults}

\section{Physical activity}

In 2014, adults in the Nordic region spent on average about five hours weekly on moderate and vigorous intensity physical activity (MVPA) during leisure time which is more than the total amount for meeting the minimum physical activity recommendations. One in three adults were classified as physically inactive in the Nordic region. Ranking of the proportions of inactive and with high recreational screen time in the five Nordic countries is shown in Table 36. The proportion of inactive was higher in Norway than in the other Nordic countries and lower in Finland and Sweden than in Denmark and Iceland.

$\begin{aligned} & \text { Table 36: Ranking from lowest to highest proportions of inactive and with high recreational screen } \\
& \text { time among adults in the five Nordic countries. NORMO 2014 }\end{aligned}$
\begin{tabular}{l|lll}
\hline Country & High recreational screen time (> 4 h/day) (\%) \\
& Inactive (\%) & Country & \\
Finland & & & 25.1 \\
Sweden & 29.1 & Sweden & 26.2 \\
Denmark & 29.7 & Iceland & 31.3 \\
Iceland & 34.3 & Norway & 33.2 \\
Norway & 35.2 & Denmark & 35.1 \\
\hline
\end{tabular}


The development from 2011 to 2014 in physical activity during leisure in the Nordic countries is shown in Table 37. Few changes were found in the Nordic region and in each of the five Nordic countries. There was an increase in the proportion of highly inactive from 2011 to 2014 in the Nordic region while no change was detected in mean MVPA and in the proportion of inactive. When each of the five Nordic countries was analysed, an increase in mean MVPA was found in Denmark, but the proportion of inactive did not change. The proportion of highly inactive increased in Iceland between 2011 and 2014 and a non-significant upward tendency was found in the other Nordic countries. This suggests that the group with almost no weekly MVPA may be rising among adults in the Nordic countries.

Moreover, the proportion of highly active increased in the Nordic region.

\section{Sedentary behaviour}

In 2014, adults in the Nordic region spent on average almost four hours daily on sedentary TV and/or computer time during leisure, which is quite high and similar to the cutoff point for high recreational screen time. Three in ten adults spent more than four hours per day on recreational screen time in the Nordic region. The proportion with high recreational screen time $(>4 \mathrm{~h} / \mathrm{d})$ was lower in Sweden and Iceland than in the other Nordic countries and higher in Finland than in Norway (Table 36 ).

Mean recreational screen time did not change from 2011 to 2014 when analysing the Nordic region, but as shown in Table 38, mixed results were found in the five Nordic countries. Between 2011 and 2014, the pattern of recreational screen time changed overall because mean TV time decreased and mean computer time increased. This is most likely a sign of smart phones and tablets have become more popular and replaced TV. Mean computer time increased systematically in all the Nordic countries, except in Norway, while changes in mean TV time were mixed in the five Nordic countries.

The proportion with high recreational screen time increased in the Nordic region from 2011 to 2014. An increase in the proportion with high recreational screen time was also found in Denmark, Finland and Iceland whereas a decrease was observed in Norway. 
Table 37: Mean and proportion (95\% Cl) for estimates of physical activity among adults in the Nordic countries. NORMO 2011 and 2014

\begin{tabular}{|c|c|c|c|c|c|c|c|c|c|c|c|c|}
\hline & \multicolumn{2}{|c|}{ Denmark } & \multicolumn{2}{|c|}{ Finland } & \multicolumn{2}{|c|}{ Sweden } & \multicolumn{2}{|c|}{ Iceland } & \multicolumn{2}{|c|}{ Norway } & \multicolumn{2}{|c|}{ Nordic region@ } \\
\hline & $\begin{array}{r}2011 \\
(n=2,093)\end{array}$ & $\begin{array}{r}2014 \\
(n=1,900)\end{array}$ & $\begin{array}{r}2011 \\
(n=1,817)\end{array}$ & $\begin{array}{r}2014 \\
(n=1,637)\end{array}$ & $\begin{array}{r}2011 \\
(n=1,772)\end{array}$ & $\begin{array}{r}2014 \\
(n=1,632)\end{array}$ & $\begin{array}{r}2011 \\
(n=1,864)\end{array}$ & $\begin{array}{r}2014 \\
(n=1,916)\end{array}$ & $\begin{array}{r}2011 \\
(n=1,011)\end{array}$ & $\begin{array}{r}2014 \\
(n=945)\end{array}$ & $\begin{array}{r}2011 \\
(n=8,557)\end{array}$ & $\begin{array}{r}2014 \\
(n=8,030)\end{array}$ \\
\hline MVPA (h/wk) & $\begin{array}{r}4.5 \\
(4 \cdot 3 ; 4.6)\end{array}$ & $\begin{array}{r}4.8^{*} \\
(4.6 ; 5.0)\end{array}$ & $\begin{array}{r}5.3 \\
(5.1 ; 5 \cdot 5)\end{array}$ & $\begin{array}{r}5 \cdot 3 \\
(5.1 ; 5.5)\end{array}$ & $\begin{array}{r}5.0 \\
(4.8 ; 5.2)\end{array}$ & $\begin{array}{r}5.2 \\
(5.0 ; 5.4)\end{array}$ & $\begin{array}{r}4 \cdot 7 \\
(4 \cdot 5 ; 4 \cdot 9)\end{array}$ & $\begin{array}{r}4.8 \\
(4.6 ; 5.0)\end{array}$ & $\begin{array}{r}4 \cdot 4 \\
(4 \cdot 1 ; 4 \cdot 7)\end{array}$ & $\begin{array}{r}4.1 \\
(3.8 ; 4.4)\end{array}$ & $\begin{array}{r}4.8 \\
(4.7 ; 4.9)\end{array}$ & $\begin{array}{r}4.9 \\
(4.8 ; 5.0)\end{array}$ \\
\hline MPA (h/wk) & $\begin{array}{r}3.0 \\
(2.8 ; 3.1)\end{array}$ & $\begin{array}{r}3.1 \\
(2.9 ; 3.2)\end{array}$ & $\begin{array}{r}3.3 \\
(3.2 ; 3.5)\end{array}$ & $\begin{array}{r}3.6^{-} \\
(3.4 ; 3.8)\end{array}$ & $\begin{array}{r}3.3 \\
(3.1 ; 3.5)\end{array}$ & $\begin{array}{r}3.1 \\
(2.9 ; 3.3)\end{array}$ & $\begin{array}{r}2.7 \\
(2.5 ; 2.8)\end{array}$ & $\begin{array}{r}2.6 \\
(2.5 ; 2.8)\end{array}$ & $\begin{array}{r}2.7 \\
(2.5 ; 2.9)\end{array}$ & $\begin{array}{r}2.9 \\
(2.7 ; 3.1)\end{array}$ & $\begin{array}{r}3.1 \\
(3.0 ; 3.2)\end{array}$ & $\begin{array}{r}3.2 \\
(3.1 ; 3.2)\end{array}$ \\
\hline VPA (h/wk) & $\begin{array}{r}1.5 \\
(1.4 ; 1.6)\end{array}$ & $\begin{array}{r}1.7^{* * *} \\
(1.6 ; 1.8)\end{array}$ & $\begin{array}{r}1.9 \\
(1.8 ; 2.0)\end{array}$ & $\begin{array}{r}1.7^{\star *} \\
(1.6 ; 1.8)\end{array}$ & $\begin{array}{r}1.8 \\
(1.7 ; 1.9)\end{array}$ & $\begin{array}{r}2.1^{* * *} \\
(1.9 ; 2.2)\end{array}$ & $\begin{array}{r}2.0 \\
(1.9 ; 2.1)\end{array}$ & $\begin{array}{r}2.1 \\
(2.0 ; 2.2)\end{array}$ & $\begin{array}{r}1.6 \\
(1.4 ; 1.7)\end{array}$ & $\begin{array}{r}1.2^{* * *} \\
(1.1 ; 1.3)\end{array}$ & $\begin{array}{r}1.7 \\
(1.7 ; 1.8)\end{array}$ & $\begin{array}{r}1.8 \\
(1.7 ; 1.8)\end{array}$ \\
\hline Inactive(\%) & $\begin{array}{r}34.4 \\
(32.4 ; 36.5)\end{array}$ & $\begin{array}{r}34.3 \\
(32.1 ; 36.4)\end{array}$ & $\begin{array}{r}28.2 \\
(26.1 ; 30.2)\end{array}$ & $\begin{array}{r}29.1 \\
(26.9 ; 31.3)\end{array}$ & $\begin{array}{r}31.2 \\
(29.0 ; 33.4)\end{array}$ & $\begin{array}{r}29.7 \\
(27.4 ; 31.9)\end{array}$ & $\begin{array}{r}34.1 \\
(32.0 ; 36.3)\end{array}$ & $\begin{array}{r}35.2 \\
(33.0 ; 37.3)\end{array}$ & $\begin{array}{r}41.2 \\
(38.1 ; 44.3)\end{array}$ & $\begin{array}{r}45.0^{-} \\
(41.9 ; 48.2)\end{array}$ & $\begin{array}{r}33.2 \\
(32.2 ; 34.2)\end{array}$ & $\begin{array}{r}33.6 \\
(32.5 ; 34.6)\end{array}$ \\
\hline $\begin{array}{l}\text { Highly inactive } \\
(\%)\end{array}$ & $\begin{array}{r}11.8 \\
(10.4 ; 13.2)\end{array}$ & $\begin{array}{r}13.4 \\
(11.8 ; 14.9)\end{array}$ & $\begin{array}{r}7.0 \\
(5.8 ; 8.2)\end{array}$ & $\begin{array}{r}8.4 \\
(7.0 ; 9.7)\end{array}$ & $\begin{array}{r}9.3 \\
(7.9 ; 10.6)\end{array}$ & $\begin{array}{r}10.9 \\
(9.4: 12.4)\end{array}$ & $\begin{array}{r}14.1 \\
(12.5 ; 15.6)\end{array}$ & $\begin{array}{r}17.4^{* *} \\
(15.7 ; 19.1)\end{array}$ & $\begin{array}{r}15.5 \\
(13.2 ; 17.7)\end{array}$ & $\begin{array}{r}18.2 \\
(15.7 ; 20.6)\end{array}$ & $\begin{array}{r}10.6 \\
(10.0 ; 11.3)\end{array}$ & $\begin{array}{r}12.4^{* * *} \\
(11.7 ; 13.1)\end{array}$ \\
\hline Active(\%) & $\begin{array}{r}65.6 \\
(63.5 ; 67.6)\end{array}$ & $\begin{array}{r}65.7 \\
(63.6 ; 67.9)\end{array}$ & $\begin{array}{r}71.8 \\
(69.8 ; 73.9)\end{array}$ & $\begin{array}{r}70.9 \\
(68.7 ; 73.1)\end{array}$ & $\begin{array}{r}68.8 \\
(66.6 ; 71.0)\end{array}$ & $\begin{array}{r}70.3 \\
(68.1 ; 72.6)\end{array}$ & $\begin{array}{r}65.9 \\
(63.7 ; 68.0)\end{array}$ & $\begin{array}{r}64.8 \\
(62.7 ; 67.0)\end{array}$ & $\begin{array}{r}58.8 \\
(55.7 ; 61.9)\end{array}$ & $\begin{array}{r}55.0^{-} \\
(51.8 ; 58.1)\end{array}$ & $\begin{array}{r}66.8 \\
(65.8 ; 67.8)\end{array}$ & $\begin{array}{r}66.4 \\
(65.4 ; 67.5)\end{array}$ \\
\hline Highly active $^{\ddagger}(\%)$ & $\begin{array}{r}11.9 \\
(10.5 ; 13.3)\end{array}$ & $\begin{array}{r}13.3 \\
(11.8 ; 14.9)\end{array}$ & $\begin{array}{r}17.6 \\
(15.9 ; 19.4)\end{array}$ & $\begin{array}{r}17.0 \\
(15.2 ; 18.9)\end{array}$ & $\begin{array}{r}13.1 \\
(11.5 ; 14.6)\end{array}$ & $\begin{array}{r}14.5 \\
(12.8 ; 16.2)\end{array}$ & $\begin{array}{r}12.2 \\
(10.7 ; 13.7)\end{array}$ & $\begin{array}{r}12.0 \\
(10.5 ; 13.5)\end{array}$ & $\begin{array}{r}10.0 \\
(8.1 ; 11.8)\end{array}$ & $\begin{array}{r}12.6^{-} \\
(10.5 ; 14.7)\end{array}$ & $\begin{array}{r}13.2 \\
(12.4 ; 13.9)\end{array}$ & $\begin{array}{r}14 \cdot 4^{*} \\
(13.6 ; 15.2)\end{array}$ \\
\hline
\end{tabular}

Note:

- $p<0.10,{ }^{*} p<0.05,{ }^{* *} p<0.01,{ }^{* * *} p<0.001$ : Differences between survey years 2011 and 2014 using t-tests and Chi-square tests.

@ Weighted according to population size in the five Nordic countries.

"Subgroup of inactive that do not add up to $100 \%$.

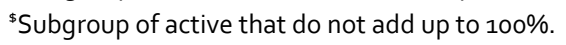


Table 38: Mean and proportion (95\% Cl) for estimates of sedentary behaviour among adults in the Nordic countries. NORMO 2011 and 2014

\begin{tabular}{|c|c|c|c|c|c|c|c|c|c|c|c|c|}
\hline & \multicolumn{2}{|c|}{ Denmark } & \multicolumn{2}{|c|}{ Finland } & \multicolumn{2}{|c|}{ Sweden } & \multicolumn{2}{|c|}{ Iceland } & \multicolumn{2}{|c|}{ Norway } & \multicolumn{2}{|c|}{ Nordic region ${ }^{\circledR}$} \\
\hline & $\begin{array}{r}2011 \\
(n=2,158)\end{array}$ & $\begin{array}{r}2014 \\
(n=1,848)\end{array}$ & $\begin{array}{r}2011 \\
(n=1,935)\end{array}$ & $\begin{array}{r}2014 \\
(n=1,579)\end{array}$ & $\begin{array}{r}2011 \\
(n=1,845)\end{array}$ & $\begin{array}{r}2014 \\
(n=1,769)\end{array}$ & $\begin{array}{r}2011 \\
(n=1,905)\end{array}$ & $\begin{array}{r}2014 \\
(n=1,889)\end{array}$ & $\begin{array}{r}2011 \\
(n=1,030)\end{array}$ & $\begin{array}{r}2014 \\
(n=1,007)\end{array}$ & $\begin{array}{r}2011 \\
(n=8,873)\end{array}$ & $\begin{array}{r}2014 \\
(n=8,092)\end{array}$ \\
\hline $\begin{array}{l}\text { Recreational } \\
\text { screen time }(\mathrm{h} / \mathrm{d})\end{array}$ & $\begin{array}{r}4.0 \\
(3.8 ; 4.1)\end{array}$ & $\begin{array}{r}4 \cdot 2^{*} \\
(4 \cdot 0 ; 4 \cdot 3)\end{array}$ & $\begin{array}{r}3.8 \\
(3.7 ; 3.9)\end{array}$ & $\begin{array}{r}4 \cdot 4^{* * *} \\
(4 \cdot 2 ; 4 \cdot 5)\end{array}$ & $\begin{array}{r}3.6 \\
(3.5 ; 3.7)\end{array}$ & $\begin{array}{r}3.4^{-} \\
(3.3 ; 3.6)\end{array}$ & $\begin{array}{r}3.4 \\
(3.3 ; 3.5)\end{array}$ & $\begin{array}{r}3.8^{\star * *} \\
(3.6 ; 3.9)\end{array}$ & $\begin{array}{r}4 \cdot 5 \\
(4 \cdot 3 ; 4 \cdot 7)\end{array}$ & $\begin{array}{r}4.0^{* * *} \\
(3.8 ; 4 \cdot 2)\end{array}$ & $\begin{array}{r}3.9 \\
(3.8 ; 4.0)\end{array}$ & $\begin{array}{r}3.9 \\
(3.8 ; 4.0)\end{array}$ \\
\hline $\operatorname{TV}(\mathrm{h} / \mathrm{d})$ & $\begin{array}{r}2.5 \\
(2.4 ; 2.6)\end{array}$ & $\begin{array}{r}2.5 \\
(2.4 ; 2.6)\end{array}$ & $\begin{array}{r}2.4 \\
(2.2 ; 2.5)\end{array}$ & $\begin{array}{r}2.6^{* *} \\
(2.5 ; 2.7)\end{array}$ & $\begin{array}{r}2.1 \\
(2.0 ; 2.2)\end{array}$ & $\begin{array}{r}1.8^{\star \star \star} \\
(1.8 ; 1.9)\end{array}$ & $\begin{array}{r}1.8 \\
(1.7 ; 1.9)\end{array}$ & $\begin{array}{r}1.8 \\
(1.7 ; 1.9)\end{array}$ & $\begin{array}{r}2.7 \\
(2.6 ; 2.9)\end{array}$ & $\begin{array}{r}2.2^{* * *} \\
(2.1 ; 2.3)\end{array}$ & $\begin{array}{r}2.4 \\
(2.3 ; 2.4)\end{array}$ & $\begin{array}{r}2.2^{* * *} \\
(2.2 ; 2.3)\end{array}$ \\
\hline Computer (h/d) & $\begin{array}{r}1.5 \\
(1.4 ; 1.5)\end{array}$ & $\begin{array}{r}1.8^{* * *} \\
(1.7 ; 1.9)\end{array}$ & $\begin{array}{r}1.5 \\
(1.4 ; 1.5)\end{array}$ & $\begin{array}{r}2.0^{* * *} \\
(1.8 ; 2.1)\end{array}$ & $\begin{array}{r}1.5 \\
(1.4 ; 1.6)\end{array}$ & $\begin{array}{r}1.6^{*} \\
(1.5 ; 1.7)\end{array}$ & $\begin{array}{r}1.7 \\
(1.6 ; 1.8)\end{array}$ & $\begin{array}{r}2.0^{* \star *} \\
(1.9 ; 2.1)\end{array}$ & $\begin{array}{r}1.8 \\
(1.7 ; 1.9)\end{array}$ & $\begin{array}{r}1.9 \\
(1.8 ; 2.0)\end{array}$ & $\begin{array}{r}1.5 \\
(1.5 ; 1.6)\end{array}$ & $\begin{array}{r}1.8^{* * *} \\
(1.8 ; 1.8)\end{array}$ \\
\hline $\begin{array}{l}\text { High recreational } \\
\text { screen time } \\
(>4 \mathrm{~h} / \mathrm{d})(\%)\end{array}$ & $\begin{array}{r}29.8 \\
(27.9 ; 31.8)\end{array}$ & $\begin{array}{r}33.2^{*} \\
(31.1 ; 35 \cdot 4)\end{array}$ & $\begin{array}{r}27.0 \\
(25.0 ; 29.0)\end{array}$ & $\begin{array}{r}35.1^{* * *} \\
(32.7 ; 37.5)\end{array}$ & $\begin{array}{r}24.9 \\
(22.9 ; 26.9)\end{array}$ & $\begin{array}{r}25.1 \\
(23.1 ; 27.1)\end{array}$ & $\begin{array}{r}22.1 \\
(20.3 ; 24.0)\end{array}$ & $\begin{array}{r}26.2^{* *} \\
(24.2 ; 28.2)\end{array}$ & $\begin{array}{r}36.5 \\
(33.6 ; 39.5)\end{array}$ & $\begin{array}{r}31.3^{*} \\
(28.4 ; 34.1)\end{array}$ & $\begin{array}{r}28.6 \\
(27.7 ; 29.5)\end{array}$ & $\begin{array}{r}30.0^{*} \\
(29.0 ; 31.0)\end{array}$ \\
\hline
\end{tabular}

$\quad \quad \quad \quad-p<0.10,{ }^{*} p<0.05,{ }^{* *} p<0.01,{ }^{* * *} p<0.001$ : Differences between survey years 2011 and 2014 using t-tests and Chi-square tests.

@Weighted according to population size in the five Nordic countries. 


\subsubsection{Gender}

\section{Physical activity}

In 2014, the proportion of inactive did not differ between genders in the Nordic region and in each of the five Nordic countries. However, the proportion of highly inactive and highly active was higher in men than in women in the Nordic region (Table 39 and 40). Further, the proportion of highly inactive was higher in men than in women in Finland, Sweden and Norway, and the proportion of highly active was higher in men than in women in Denmark.

Data showed no change in mean MVPA from 2011 to 2014 in men and women in the Nordic region. But mean MVPA increased in women in Denmark and Finland due to an increase in mean MPA. 
Table 39: Mean and proportion $(95 \% \mathrm{Cl})$ and for estimates of physical activity among men in the Nordic countries. NORMO 2011 and 2014

\begin{tabular}{|c|c|c|c|c|c|c|c|c|c|c|c|c|}
\hline & \multicolumn{2}{|c|}{ Denmark } & \multicolumn{2}{|c|}{ Finland } & \multicolumn{2}{|c|}{ Sweden } & \multicolumn{2}{|c|}{ Iceland } & \multicolumn{2}{|c|}{ Norway } & \multicolumn{2}{|c|}{ Nordic region@ } \\
\hline & $\begin{array}{r}2011 \\
(n=960)\end{array}$ & $\begin{array}{r}2014 \\
(n=917)\end{array}$ & $\begin{array}{r}2011 \\
(n=818)\end{array}$ & $\begin{array}{r}2014 \\
(n=713)\end{array}$ & $\begin{array}{r}2011 \\
(n=831)\end{array}$ & $\begin{array}{r}2014 \\
(n=732)\end{array}$ & $\begin{array}{r}2011 \\
(n=864)\end{array}$ & $\begin{array}{r}2014 \\
(n=845)\end{array}$ & $\begin{array}{r}2011 \\
(n=482)\end{array}$ & $\begin{array}{r}2014 \\
(n=431)\end{array}$ & $\begin{array}{r}2011 \\
(n=3,955)\end{array}$ & $\begin{array}{r}2014 \\
(n=3,638)\end{array}$ \\
\hline MVPA (h/wk) & $\begin{array}{r}4.6 \\
(4 \cdot 3 i 4 \cdot 9)\end{array}$ & $\begin{array}{r}4.9 \\
(4.6 ; 5.2)\end{array}$ & $\begin{array}{r}5.6 \\
(5.2 ; 5.9)\end{array}$ & $\begin{array}{r}5.1^{-} \\
(4.8 ; 5 \cdot 4)\end{array}$ & $\begin{array}{r}5.0 \\
(4.775 .4)\end{array}$ & $\begin{array}{r}5.3 \\
(5.0 ; 5.6)\end{array}$ & $\begin{array}{r}5.0 \\
(4.775 \cdot 3)\end{array}$ & $\begin{array}{r}5.0 \\
(4.6 ; 5 \cdot 3)\end{array}$ & $\begin{array}{r}4.5 \\
(4.0 ; 4.9)\end{array}$ & $\begin{array}{r}4.3 \\
(3.8 ; 4.7)\end{array}$ & $\begin{array}{r}4.9 \\
(4.8 ; 5.1)\end{array}$ & $\begin{array}{r}4.9 \\
(4.8 ; 5 \cdot 1)\end{array}$ \\
\hline MPA (h/wk) & $\begin{array}{r}3.0 \\
(2.773 .2)\end{array}$ & $\begin{array}{r}2.8 \\
(2.6 ; 3.0)\end{array}$ & $\begin{array}{r}3.3 \\
(3.0 ; 3.6)\end{array}$ & $\begin{array}{r}3.3 \\
(3.0 ; 3.5)\end{array}$ & $\begin{array}{r}3.2 \\
(2.9 ; 3.5)\end{array}$ & $\begin{array}{r}3.0 \\
(2.733 .2)\end{array}$ & $\begin{array}{r}2.8 \\
(2.5 ; 3.0)\end{array}$ & $\begin{array}{r}2.6 \\
(2.4 ; 2.9)\end{array}$ & $\begin{array}{r}2.5 \\
(2.2 ; 2.8)\end{array}$ & $\begin{array}{r}3.1^{*} \\
(2.7 ; 3.5)\end{array}$ & $\begin{array}{r}3.0 \\
(2.9 ; 3.2)\end{array}$ & $\begin{array}{r}3.0 \\
(2.9 ; 3.1)\end{array}$ \\
\hline VPA (h/wk) & $\begin{array}{r}1.6 \\
(1.5 ; 1.8)\end{array}$ & $\begin{array}{r}2.1^{* * *} \\
(1.9 ; 2.2)\end{array}$ & $\begin{array}{r}2.2 \\
(2.1 ; 2.4)\end{array}$ & $\begin{array}{r}1.8^{* *} \\
(1.7 ; 2.0)\end{array}$ & $\begin{array}{r}1.9 \\
(1.7 ; 2.0)\end{array}$ & $\begin{array}{r}2.3^{* *} \\
(2.1 ; 2.5)\end{array}$ & $\begin{array}{r}2.2 \\
(2.0 ; 2.4)\end{array}$ & $\begin{array}{r}2.3 \\
(2.1 ; 2.5)\end{array}$ & $\begin{array}{r}1.7 \\
(1.5 ; 1.9)\end{array}$ & $\begin{array}{r}1.2^{* * *} \\
(1.0 ; 1.4)\end{array}$ & $\begin{array}{r}1.9 \\
(1.8 ; 1.9)\end{array}$ & $\begin{array}{r}1.9 \\
(1.8 ; 2.0)\end{array}$ \\
\hline Inactive(\%) & $\begin{array}{r}35.6 \\
(32.5 ; 38.6)\end{array}$ & $\begin{array}{r}34.6 \\
(31.5 ; 37.7)\end{array}$ & $\begin{array}{r}27.4 \\
(24.4 ; 30.5)\end{array}$ & $\begin{array}{r}31.2 \\
(27.8 ; 34.6)\end{array}$ & $\begin{array}{r}33.5 \\
(30.3 ; 36.7)\end{array}$ & $\begin{array}{r}28.9^{*} \\
(25.6 ; 32.2)\end{array}$ & $\begin{array}{r}33.9 \\
(30.7 ; 37.0)\end{array}$ & $\begin{array}{r}35.9 \\
(32.7 ; 39.2)\end{array}$ & $\begin{array}{r}40.3 \\
(35.9 ; 44.7)\end{array}$ & $\begin{array}{r}46.0^{-} \\
(41.2 ; 50.7)\end{array}$ & $\begin{array}{r}34.1 \\
(32.6 ; 35.6)\end{array}$ & $\begin{array}{r}34.0 \\
(32.5 ; 35.6)\end{array}$ \\
\hline $\begin{array}{l}\text { Highly inactive" } \\
(\%)\end{array}$ & $\begin{array}{r}13.6 \\
(11.5 ; 15.8)\end{array}$ & $\begin{array}{r}14.4 \\
(12.1 ; 16.6)\end{array}$ & $\begin{array}{r}8.7 \\
(6.7 ; 10.6)\end{array}$ & $\begin{array}{r}10.4 \\
(8.1 ; 12.6)\end{array}$ & $\begin{array}{r}11.6 \\
(9.4 ; 13.8)\end{array}$ & $\begin{array}{r}12.5 \\
(10.1: 14.9)\end{array}$ & $\begin{array}{r}14.7 \\
(12.3 ; 17.0)\end{array}$ & $\begin{array}{r}18.6^{*} \\
(16.0 ; 21.2)\end{array}$ & $\begin{array}{r}17.7 \\
(14.2 ; 21.1)\end{array}$ & $\begin{array}{r}20.9 \\
(17.1 ; 24.8)\end{array}$ & $\begin{array}{r}12.7 \\
(11.6 ; 13.7)\end{array}$ & $\begin{array}{r}14.2 \\
(13.0 ; 15.3)\end{array}$ \\
\hline Active(\%) & $\begin{array}{r}64.4 \\
(61.4 ; 67.5)\end{array}$ & $\begin{array}{r}65.4 \\
(62.3 ; 68.5)\end{array}$ & $\begin{array}{r}72.6 \\
(69.5 ; 75.6)\end{array}$ & $\begin{array}{r}68.8 \\
(65.4 ; 72.2)\end{array}$ & $\begin{array}{r}66.5 \\
(63.3 ; 69.7)\end{array}$ & $\begin{array}{r}71.1^{*} \\
(67.8 ; 74.4)\end{array}$ & $\begin{array}{r}66.1 \\
(63.0 ; 69.3)\end{array}$ & $\begin{array}{r}64.1 \\
(60.8 ; 67.3)\end{array}$ & $\begin{array}{r}59.7 \\
(55 \cdot 3 ; 64.1)\end{array}$ & $\begin{array}{r}54.0^{-} \\
(49.3 ; 58.8)\end{array}$ & $\begin{array}{r}65.9 \\
(64.4 ; 67.4)\end{array}$ & $\begin{array}{r}66.0 \\
(64.4 ; 67.5)\end{array}$ \\
\hline Highly actives (\%) & $\begin{array}{r}13.0 \\
(10.8 ; 15.1)\end{array}$ & $\begin{array}{r}15.6^{-} \\
(13.3 ; 18.0)\end{array}$ & $\begin{array}{r}18.4 \\
(15.7 ; 21.0)\end{array}$ & $\begin{array}{r}16.6 \\
(13.9 ; 19.4)\end{array}$ & $\begin{array}{r}12.5 \\
(10.2 ; 14.7)\end{array}$ & $\begin{array}{r}15.1 \\
(12.5 ; 17.7)\end{array}$ & $\begin{array}{r}14.0 \\
(11.7 ; 16.3)\end{array}$ & $\begin{array}{r}12.3 \\
(10.1 ; 14.5)\end{array}$ & $\begin{array}{r}8.9 \\
(6.3 ; 11.5)\end{array}$ & $\begin{array}{r}13.9^{*} \\
(10.6 ; 17.2)\end{array}$ & $\begin{array}{r}13.1 \\
(12.0 ; 14.1)\end{array}$ & $\begin{array}{r}15 \cdot 3^{*+} \\
(14 \cdot 1 ; 16.5)\end{array}$ \\
\hline
\end{tabular}

Note:

- $p<0.10,{ }^{*} p<0.05,{ }^{* *} p<0.01,{ }^{* * *} p<0.001$ : Differences between survey years 2011 and 2014 using t-tests and Chi-square tests. @Weighted according to population size in the five Nordic countries.

\#Subgroup of inactive that do not add up to $100 \%$.

sSubgroup of active that do not add up to $100 \%$. 
Table 40: Mean and proportion (95\% Cl) for estimates of physical activity among women in the Nordic countries. NORMO 2011 and 2014

\begin{tabular}{|c|c|c|c|c|c|c|c|c|c|c|c|c|}
\hline & \multicolumn{2}{|c|}{ Denmark } & \multicolumn{2}{|c|}{ Finland } & \multicolumn{2}{|c|}{ Sweden } & \multicolumn{2}{|c|}{ Iceland } & \multicolumn{2}{|c|}{ Norway } & \multicolumn{2}{|c|}{ Nordic region@ } \\
\hline & $\begin{array}{r}2011 \\
(n=1,133)\end{array}$ & $\begin{array}{r}2014 \\
(n=982)\end{array}$ & $\begin{array}{r}2011 \\
(n=999)\end{array}$ & $\begin{array}{r}2014 \\
(n=924)\end{array}$ & $\begin{array}{r}2011 \\
(n=941)\end{array}$ & $\begin{array}{r}2014 \\
(n=900)\end{array}$ & $\begin{array}{r}2011 \\
(n=1,000)\end{array}$ & $\begin{array}{r}2014 \\
(n=1,071)\end{array}$ & $\begin{array}{r}2011 \\
(n=529)\end{array}$ & $\begin{array}{r}2014 \\
(n=514)\end{array}$ & $\begin{array}{r}2011 \\
(n=4,602)\end{array}$ & $\begin{array}{r}2014 \\
(n=4,391)\end{array}$ \\
\hline MVPA (h/wk) & $\begin{array}{r}4 \cdot 3 \\
(4.1 ; 4 \cdot 6)\end{array}$ & $\begin{array}{r}4.8^{*} \\
(4 \cdot 5 ; 5 \cdot 0)\end{array}$ & $\begin{array}{r}5.0 \\
(4.8 ; 5 \cdot 3)\end{array}$ & $\begin{array}{r}5.5^{*} \\
(5.2 ; 5.8)\end{array}$ & $\begin{array}{r}5.0 \\
(4.7 ; 5.3)\end{array}$ & $\begin{array}{r}5.1 \\
(4.8 ; 5.4)\end{array}$ & $\begin{array}{r}4 \cdot 3 \\
(4 \cdot 1 ; 4 \cdot 5)\end{array}$ & $\begin{array}{r}4.6 \\
(4.3 ; 4.8)\end{array}$ & $\begin{array}{r}4.4 \\
(4.0 ; 4.8)\end{array}$ & $\begin{array}{r}4.0 \\
(3.6 ; 4.3)\end{array}$ & $\begin{array}{r}4.7 \\
(4.6 ; 4.9)\end{array}$ & $\begin{array}{r}4.9 \\
(4.8 ; 5.0)\end{array}$ \\
\hline MPA (h/wk) & $\begin{array}{r}3.0 \\
(2.8 ; 3.2)\end{array}$ & $\begin{array}{r}3.3^{*} \\
(3.1 ; 3.6)\end{array}$ & $\begin{array}{r}3.4 \\
(3.1 ; 3.6)\end{array}$ & $\begin{array}{r}3.9^{* *} \\
(3.6 ; 4.1)\end{array}$ & $\begin{array}{r}3.4 \\
(3.1 ; 3.6)\end{array}$ & $\begin{array}{r}3.3 \\
(3.0 ; 3.5)\end{array}$ & $\begin{array}{r}2.6 \\
(2.4 ; 2.8)\end{array}$ & $\begin{array}{r}2.6 \\
(2.4 ; 2.9)\end{array}$ & $\begin{array}{r}2.8 \\
(2.5 ; 3.1)\end{array}$ & $\begin{array}{r}2.7 \\
(2.4 ; 3.0)\end{array}$ & $\begin{array}{r}3.2 \\
(3.1 ; 3 \cdot 3)\end{array}$ & $\begin{array}{r}3.3 \\
(3.2 ; 3.4\end{array}$ \\
\hline VPA (h/wk) & $\begin{array}{r}1.3 \\
(1.2 ; 1.4)\end{array}$ & $\begin{array}{r}1.4 \\
(1.3 ; 1.5)\end{array}$ & $\begin{array}{r}1.7 \\
(1.6 ; 1.8)\end{array}$ & $\begin{array}{r}1.6 \\
(1.5 ; 1.8)\end{array}$ & $\begin{array}{r}1.7 \\
(1.5 ; 1.8)\end{array}$ & $\begin{array}{r}1.8 \\
(1.7 ; 2.0)\end{array}$ & $\begin{array}{r}1.8 \\
(1.7 ; 1.9)\end{array}$ & $\begin{array}{r}1.9 \\
(1.8 ; 2.0)\end{array}$ & $\begin{array}{r}1.5 \\
(1.3 ; 1.6)\end{array}$ & $\begin{array}{r}1.2^{-} \\
(1.1 ; 1.4)\end{array}$ & $\begin{array}{r}1.5 \\
(1.5 ; 1.6)\end{array}$ & $\begin{array}{r}1.6 \\
(1.5 ; 1.6)\end{array}$ \\
\hline Inactive(\%) & $\begin{array}{r}33.3 \\
(30.6 ; 36.0)\end{array}$ & $\begin{array}{r}33.9 \\
(31.0 ; 36.9)\end{array}$ & $\begin{array}{r}28.8 \\
(26.0 ; 31.6)\end{array}$ & $\begin{array}{r}27.2 \\
(24.3 ; 30.1)\end{array}$ & $\begin{array}{r}28.8 \\
(25.8 ; 31.7)\end{array}$ & $\begin{array}{r}30.4 \\
(27.4 ; 33.4)\end{array}$ & $\begin{array}{r}34.4 \\
(31.5 ; 37.3)\end{array}$ & $\begin{array}{r}34.4 \\
(31.6 ; 37.3)\end{array}$ & $\begin{array}{r}42.0 \\
(37.8 ; 46.3)\end{array}$ & $\begin{array}{r}44.2 \\
(39.9 ; 48.4)\end{array}$ & $\begin{array}{r}32.4 \\
(31.0 ; 33.7)\end{array}$ & $\begin{array}{r}33.1 \\
(31.7 ; 34.5)\end{array}$ \\
\hline $\begin{array}{l}\text { Highly inactive }{ }^{\#} \\
(\%)\end{array}$ & $\begin{array}{r}10.0 \\
(8.2 ; 11.7)\end{array}$ & $\begin{array}{r}12.4^{-} \\
(10.3 ; 14.5)\end{array}$ & $\begin{array}{r}5.5 \\
(4.1 ; 6.9)\end{array}$ & $\begin{array}{r}6.5 \\
(4.9 ; 8.1)\end{array}$ & $\begin{array}{r}6.8 \\
(5.2 ; 8.5)\end{array}$ & $\begin{array}{r}9 \cdot 4^{*} \\
(7.5: 11.3)\end{array}$ & $\begin{array}{r}13.4 \\
(11.3 ; 15.6)\end{array}$ & $\begin{array}{r}16.3^{-} \\
(14.1 ; 18.5)\end{array}$ & $\begin{array}{r}13.3 \\
(10.3 ; 16.2)\end{array}$ & $\begin{array}{r}15.5 \\
(12.4 ; 18.6)\end{array}$ & $\begin{array}{r}8.6 \\
(7.7 ; 9.4)\end{array}$ & $\begin{array}{r}10.6^{* * *} \\
(9.7 ; 11.6)\end{array}$ \\
\hline Active(\%) & $\begin{array}{r}66.7 \\
(64.0 ; 69.4)\end{array}$ & $\begin{array}{r}66.1 \\
(63.1 ; 69.0)\end{array}$ & $\begin{array}{r}71.2 \\
(68.4 ; 74.0)\end{array}$ & $\begin{array}{r}72.8 \\
(69.9 ; 75.7)\end{array}$ & $\begin{array}{r}71.2 \\
(68.3 ; 74.2)\end{array}$ & $\begin{array}{r}69.6 \\
(66.6 ; 72.6)\end{array}$ & $\begin{array}{r}65.6 \\
(62.6 ; 68.5)\end{array}$ & $\begin{array}{r}65.6 \\
(62.7 ; 68.4)\end{array}$ & $\begin{array}{r}58.0 \\
(53.7 ; 62.2)\end{array}$ & $\begin{array}{r}55.8 \\
(51.6 ; 60.1)\end{array}$ & $\begin{array}{r}67.6 \\
(66.3 ; 69.0)\end{array}$ & $\begin{array}{r}66.9 \\
(65.5 ; 68.3)\end{array}$ \\
\hline Highly actives $(\%)$ & $\begin{array}{r}10.9 \\
(9.0 ; 12.7)\end{array}$ & $\begin{array}{r}11.0 \\
(9.1 ; 13.0)\end{array}$ & $\begin{array}{r}17.0 \\
(14.7 ; 19.3)\end{array}$ & $\begin{array}{r}17.4 \\
(14.9 ; 19.8)\end{array}$ & $\begin{array}{r}13.7 \\
(11.4 ; 15.9)\end{array}$ & $\begin{array}{r}13.8 \\
(11.6 ; 16.1)\end{array}$ & $\begin{array}{r}10.4 \\
(8.5 ; 12.3)\end{array}$ & $\begin{array}{r}11.7 \\
(9.8 ; 13.7)\end{array}$ & $\begin{array}{r}11.1 \\
(8.4 ; 13.8)\end{array}$ & $\begin{array}{r}11.4 \\
(8.6 ; 14.1)\end{array}$ & $\begin{array}{r}13.2 \\
(12.2 ; 14.2)\end{array}$ & $\begin{array}{r}13.5 \\
(12.5 ; 14.6)\end{array}$ \\
\hline
\end{tabular}

$\quad \quad \quad-p<0.10,{ }^{*} p<0.05,{ }^{* *} p<0.01,{ }^{* * *} p<0.001$ : Differences between survey years 2011 and 2014 using t-tests and Chi-square tests.

@Weighted according to population size in the five Nordic countries.

"Subgroup of inactive that do not add up to $100 \%$.

${ }^{\text {s }}$ Subgroup of active that do not add up to $100 \%$. 
Between 2011 and 2014, in the Nordic region, the proportion of highly inactive increased in women and tended to increase in men. No change was observed in the proportion of inactive in both genders between 2011 and 2014. When analysing each of the five Nordic countries, the proportion of inactive decreased in men in Sweden while no change was found in women. Furthermore, an upward tendency from 2011 to 2014 was found in the proportion of highly inactive in both men and women in all the Nordic countries. The increase was significant in men in Iceland and in women in Sweden.

Finally, the proportion of highly active increased in men in the Nordic region and in Norway.

Sedentary behaviour

In 2014, the proportion with high recreational screen time was higher in men than in women in the Nordic region ( $32.7 \%$ vs. $27.3 \%$ ). This gender difference was also found in Finland, Sweden and Norway.

Between 2011 and 2014, mean recreational screen time did not change in men and women in the Nordic region while mixed results were found when the development in each of the five countries was examined (Table 41 and 42). However, the pattern of recreational screen time changed from 2011 to 2014 in men and women in the Nordic region: TV time declined and computer time rose.

In the Nordic region, data from 2011 to 2014 showed no change in the proportion with high recreational screen time in men and women. Analyses in each of the five Nordic countries showed an increase in the proportion with high recreational screen time in men in Finland and in women in Denmark, Finland and Iceland. Hence, data showed an increase in the proportion with high recreational screen time in men and/or women in Denmark, Finland and Iceland, but not in Sweden and Norway. 
Table 41: Mean and proportion ( $95 \% \mathrm{Cl}$ ) and for estimates of sedentary behaviour among men in the Nordic countries. NORMO 2011 and 2014

\begin{tabular}{|c|c|c|c|c|c|c|c|c|c|c|c|c|}
\hline & \multicolumn{2}{|c|}{ Denmark } & \multicolumn{2}{|c|}{ Finland } & \multicolumn{2}{|c|}{ Sweden } & \multicolumn{2}{|c|}{ Iceland } & \multicolumn{2}{|c|}{ Norway } & \multicolumn{2}{|c|}{ Nordic region@ } \\
\hline & $\begin{array}{r}2011 \\
(n=999)\end{array}$ & $\begin{array}{r}2014 \\
(n=889)\end{array}$ & $\begin{array}{r}2011 \\
(n=888)\end{array}$ & $\begin{array}{r}2014 \\
(n=686)\end{array}$ & $\begin{array}{r}2011 \\
(n=862)\end{array}$ & $\begin{array}{r}2014 \\
(n=811)\end{array}$ & $\begin{array}{r}2011 \\
(n=888)\end{array}$ & $\begin{array}{r}2014 \\
(n=842)\end{array}$ & $\begin{array}{r}2011 \\
(n=499)\end{array}$ & $\begin{array}{r}2014 \\
(n=462)\end{array}$ & $\begin{array}{r}2011 \\
(n=4,136)\end{array}$ & $\begin{array}{r}2014 \\
(n=3,690)\end{array}$ \\
\hline $\begin{array}{l}\text { Recreational } \\
\text { screen time }(\mathrm{h} / \mathrm{d})\end{array}$ & $\begin{array}{r}4.1 \\
(3.9 ; 4 \cdot 3)\end{array}$ & $\begin{array}{r}4.1 \\
(3.9 ; 4 \cdot 3)\end{array}$ & $\begin{array}{r}3.9 \\
(3.7 ; 4.2)\end{array}$ & $\begin{array}{r}4 \cdot 7^{* * *} \\
(4 \cdot 4 ; 5.0)\end{array}$ & $\begin{array}{r}3.7 \\
(3.5 ; 3.9)\end{array}$ & $\begin{array}{r}3.7 \\
(3.5 ; 3.9)\end{array}$ & $\begin{array}{r}3.6 \\
(3.4 ; 3.7)\end{array}$ & $\begin{array}{r}3.8 \\
(3.6 ; 4.0)\end{array}$ & $\begin{array}{r}4.9 \\
(4.5 ; 5.2)\end{array}$ & $\begin{array}{r}4 \cdot 2^{* *} \\
(3 \cdot 9 \cdot 4 \cdot 5)\end{array}$ & $\begin{array}{r}4.1 \\
(4.0 ; 4.2)\end{array}$ & $\begin{array}{r}4 \cdot 1 \\
(4.0 ; 4 \cdot 2)\end{array}$ \\
\hline $\operatorname{TV}(\mathrm{h} / \mathrm{d})$ & $\begin{array}{r}2.5 \\
(2.3 ; 2.6)\end{array}$ & $\begin{array}{r}2.4 \\
(2.3 ; 2.6)\end{array}$ & $\begin{array}{r}2.4 \\
(2.2 ; 2.6)\end{array}$ & $\begin{array}{r}2.8^{* *} \\
(2.6 ; 3.0)\end{array}$ & $\begin{array}{r}2.1 \\
(2.0 ; 2.2)\end{array}$ & $\begin{array}{r}2.0 \\
(1.8 ; 2.1)\end{array}$ & $\begin{array}{r}1.8 \\
(1.7 ; 2.0)\end{array}$ & $\begin{array}{r}1.8 \\
(1.7 ; 1.9)\end{array}$ & $\begin{array}{r}2.9 \\
(2.6 ; 3.1)\end{array}$ & $\begin{array}{r}2.3^{* * *} \\
(2.1 ; 2.5)\end{array}$ & $\begin{array}{r}2.4 \\
(2.3 ; 2.5)\end{array}$ & $\begin{array}{r}2.3^{*} \\
(2.2 ; 2.4)\end{array}$ \\
\hline Computer (h/d) & $\begin{array}{r}1.6 \\
(1.5 ; 1.8)\end{array}$ & $\begin{array}{r}1.8^{*} \\
(1.7 ; 2.0)\end{array}$ & $\begin{array}{r}1.6 \\
(1.4 ; 1.7)\end{array}$ & $\begin{array}{r}2.2^{* * *} \\
(2.0 ; 2.3)\end{array}$ & $\begin{array}{r}1.6 \\
(1.4 ; 1.7)\end{array}$ & $\begin{array}{r}1.8^{*} \\
(1.6 ; 1.9)\end{array}$ & $\begin{array}{r}1.8 \\
(1.6 ; 1.9)\end{array}$ & $\begin{array}{r}2.0^{*} \\
(1.9 ; 2.2)\end{array}$ & $\begin{array}{r}2.0 \\
(1.8 ; 2.2)\end{array}$ & $\begin{array}{r}2.0 \\
(1.8 ; 2.2)\end{array}$ & $\begin{array}{r}1.7 \\
(1.6 ; 1.7)\end{array}$ & $\begin{array}{r}1.9^{* * *} \\
(1.9 ; 2.0)\end{array}$ \\
\hline $\begin{array}{l}\text { High recreational } \\
\text { screen time (>4 } \\
\text { h/d) (\%) }\end{array}$ & $\begin{array}{r}34.0 \\
(31.0 ; 36.9)\end{array}$ & $\begin{array}{r}32.4 \\
(29 \cdot 3 ; 35 \cdot 5)\end{array}$ & $\begin{array}{r}28.6 \\
(25.7 ; 31.6)\end{array}$ & $\begin{array}{r}40.7^{* * *} \\
(37.0 ; 44.4)\end{array}$ & $\begin{array}{r}25.9 \\
(23.0 ; 28.8)\end{array}$ & $\begin{array}{r}28.0 \\
(24.9 ; 31.0)\end{array}$ & $\begin{array}{r}23.1 \\
(20.4 ; 25.9)\end{array}$ & $\begin{array}{r}26.3 \\
(23.3 ; 29.3)\end{array}$ & $\begin{array}{r}40.6 \\
(36.3 ; 45.0)\end{array}$ & $\begin{array}{r}35.0^{-} \\
(30.6 ; 39.4)\end{array}$ & $\begin{array}{r}31.1 \\
(29.7 ; 32.5)\end{array}$ & $\begin{array}{r}32.7 \\
(31.2 ; 34.2)\end{array}$ \\
\hline
\end{tabular}

$\quad \quad \quad-p<0.10,{ }^{*} p<0.05{ }^{* *} p<0.01,{ }^{* * *} p<0.001$ : Differences between survey years 2011 and 2014 using t-tests and Chi-square tests. @Weighted according to population size in the five Nordic countries.

Table 42: Mean and proportion $(95 \% \mathrm{Cl})$ for estimates of sedentary behaviour among women in the Nordic countries. NORMO 2011 and 2014

\begin{tabular}{|c|c|c|c|c|c|c|c|c|c|c|c|c|}
\hline & \multicolumn{2}{|c|}{ Denmark } & \multicolumn{2}{|c|}{ Finland } & \multicolumn{2}{|c|}{ Sweden } & \multicolumn{2}{|c|}{ Iceland } & \multicolumn{2}{|c|}{ Norway } & \multicolumn{2}{|c|}{ Nordic region@ } \\
\hline & $\begin{array}{r}2011 \\
(n=1,159)\end{array}$ & $\begin{array}{r}2014 \\
(n=958)\end{array}$ & $\begin{array}{r}2011 \\
(n=1,047)\end{array}$ & $\begin{array}{r}2014 \\
(n=893)\end{array}$ & $\begin{array}{r}2011 \\
(n=983)\end{array}$ & $\begin{array}{r}2014 \\
(n=958)\end{array}$ & $\begin{array}{r}2011 \\
(n=1,017)\end{array}$ & $\begin{array}{r}2014 \\
(n=1,047)\end{array}$ & $\begin{array}{r}2011 \\
(n=531)\end{array}$ & $\begin{array}{r}2014 \\
(n=545)\end{array}$ & $\begin{array}{r}2011 \\
(n=4,737)\end{array}$ & $\begin{array}{r}2014 \\
(n=4,401)\end{array}$ \\
\hline $\begin{array}{l}\text { Recreational } \\
\text { screen time }(h / d)\end{array}$ & $\begin{array}{r}3.8 \\
(3.6 ; 3.9)\end{array}$ & $\begin{array}{r}4 \cdot 2^{* * *} \\
(4 \cdot 0 ; 4 \cdot 4)\end{array}$ & $\begin{array}{r}3.7 \\
(3.5 i 3.9)\end{array}$ & $\begin{array}{r}4 \cdot 1^{* *} \\
(3 \cdot 9 ; 4 \cdot 3)\end{array}$ & $\begin{array}{r}3.5 \\
(3.3 ; 3.7)\end{array}$ & $\begin{array}{r}3.2^{* *} \\
(3.0 ; 3.4)\end{array}$ & $\begin{array}{r}3.3 \\
(3.1 ; 3.4)\end{array}$ & $\begin{array}{r}3.7^{\star * *} \\
(3.6 ; 3.9)\end{array}$ & $\begin{array}{r}4.1 \\
(3.9 ; 4 \cdot 4)\end{array}$ & $\begin{array}{r}3.8^{-} \\
(3.6 ; 4.1)\end{array}$ & $\begin{array}{r}3.7 \\
(3.6 ; 3.8)\end{array}$ & $\begin{array}{r}3.7 \\
(3.6 ; 3.8)\end{array}$ \\
\hline $\mathrm{TV}(\mathrm{h} / \mathrm{d})$ & $\begin{array}{r}2.5 \\
(2.4 ; 2.6)\end{array}$ & $\begin{array}{r}2.6 \\
(2.4 ; 2.8)\end{array}$ & $\begin{array}{r}2.3 \\
(2.1 ; 2.4)\end{array}$ & $\begin{array}{r}2.4 \\
(2.3 ; 2.6)\end{array}$ & $\begin{array}{r}2.1 \\
(2.0 ; 2.3)\end{array}$ & $\begin{array}{r}1.7^{* * *} \\
(1.6 ; 1.8)\end{array}$ & $\begin{array}{r}1.8 \\
(1.7 ; 1.9)\end{array}$ & $\begin{array}{r}1.9 \\
(1.7 ; 2.0)\end{array}$ & $\begin{array}{r}2.5 \\
(2.3 ; 2.7)\end{array}$ & $\begin{array}{r}2.1^{* *} \\
(2.0 ; 2.3)\end{array}$ & $\begin{array}{r}2.3 \\
(2.3 ; 2.4)\end{array}$ & $\begin{array}{r}2.1^{* \star \star} \\
(2.1 ; 2.2)\end{array}$ \\
\hline Computer (h/d) & $\begin{array}{r}1.3 \\
(1.2 ; 1.4)\end{array}$ & $\begin{array}{r}1.8^{* * *} \\
(1.7 ; 2.0)\end{array}$ & $\begin{array}{r}1.4 \\
(1.3 ; 1.5)\end{array}$ & $\begin{array}{r}1.8^{* * *} \\
(1.6 ; 1.9)\end{array}$ & $\begin{array}{r}1.4 \\
(1.3 ; 1.5)\end{array}$ & $\begin{array}{r}1.5 \\
(1.4 ; 1.6)\end{array}$ & $\begin{array}{r}1.6 \\
(1.5 ; 1.7)\end{array}$ & $\begin{array}{r}1.9^{* * *} \\
(1.8 ; 2.1)\end{array}$ & $\begin{array}{r}1.6 \\
(1.4 ; 1.8)\end{array}$ & $\begin{array}{r}1.8 \\
(1.6 ; 2.0)\end{array}$ & $\begin{array}{r}1.4 \\
(1.4 ; 1.5)\end{array}$ & $\begin{array}{r}1.7^{* * \star} \\
(1.6 ; 1.7)\end{array}$ \\
\hline $\begin{array}{l}\text { High recreational } \\
\text { screen time (> } 4 \\
\text { h/d) (\%) }\end{array}$ & $\begin{array}{r}25.7 \\
(23.2 ; 28.2)\end{array}$ & $\begin{array}{r}34 \cdot 1^{* * *} \\
(31 \cdot 1 ; 37 \cdot 1)\end{array}$ & $\begin{array}{r}25.5 \\
(22.9 ; 28.1)\end{array}$ & $\begin{array}{r}30.0^{*} \\
(27.0 ; 33.0)\end{array}$ & $\begin{array}{r}23.9 \\
(21.2 ; 26.5)\end{array}$ & $\begin{array}{r}22.1 \\
(19.5 ; 24.8)\end{array}$ & $\begin{array}{r}21.1 \\
(18.6 ; 23.6)\end{array}$ & $\begin{array}{r}26.0^{* *} \\
(23.4 ; 28.7)\end{array}$ & $\begin{array}{r}32.2 \\
(28.2 ; 36.2)\end{array}$ & $\begin{array}{r}27.5^{-} \\
(23.8 ; 31.3)\end{array}$ & $\begin{array}{r}26.1 \\
(24.9 ; 27.4)\end{array}$ & $\begin{array}{r}27.3 \\
(26.0 ; 28.6)\end{array}$ \\
\hline
\end{tabular}

$\quad$ Note: $\quad-p<0.10,{ }^{*} p<0.05,{ }^{* *} p<0.01,{ }^{* * *} p<0.001$ : Differences between survey years 2011 and 2014 using t-tests and Chi-square tests.

@Weighted according to population size in the five Nordic countries. 


\subsubsection{Age}

\section{Physical activity}

In 2014, the proportion of inactive was higher in 25-44- and 45-65-y-olds than in 18$24-y$-olds in the Nordic region. Similar results were found in Denmark. In Sweden, a higher proportion of inactive was seen in $45-65$-y-olds than in $18-24$-y-olds, while a higher proportion of inactive was found in $45-65$-y-olds than in $25-44$-y-olds in Iceland. In Norway, a higher proportion of inactive was observed in $25-44^{-} y$-olds than in $45^{-6}$ $y$-olds. No difference between age groups was seen in Finland.

Data in the three age groups displayed no change in mean MVPA between 2011 and 2014 when analysing the Nordic region (Table 43). Analyses in each of the five Nordic countries showed that mean MVPA increased in 45-65-y-olds in Denmark.

Table 43: Mean and proportion $(95 \% \mathrm{Cl})$ for estimates of physical activity among adults according to age in the Nordic region. NORMO 2011 and 2014

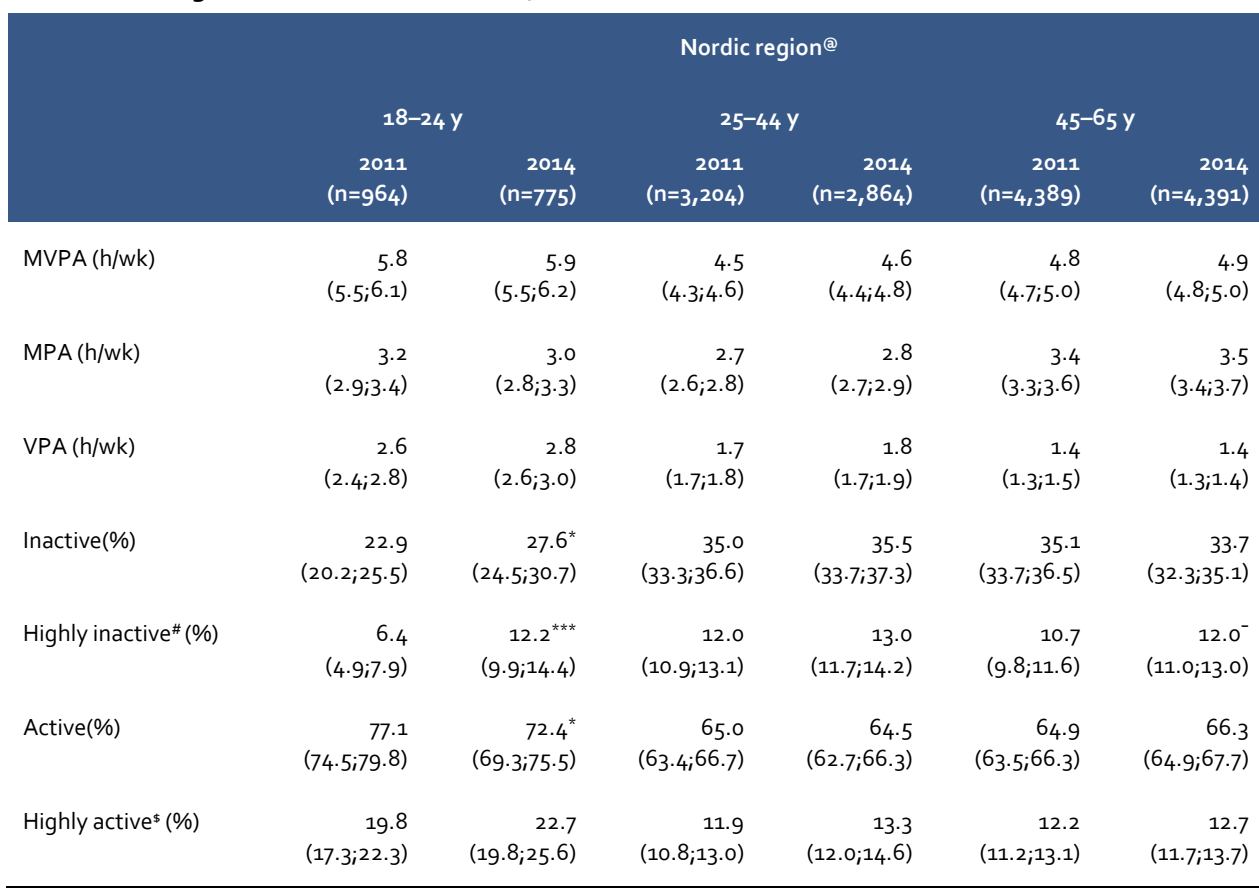

Note: $\quad-p<0.10,{ }^{*} p<0.05,{ }^{* * *} p<0.001$ : Differences between survey years 2011 and 2014 using t-tests and Chisquare tests.

\#Subgroup of inactive that do not add up to $100 \%$.

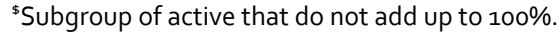

@Weighted according to population size in the five Nordic countries. 
Between 2011 and 2014, the proportions of inactive and highly inactive increased in 18$24-y$-olds in the Nordic region. The proportion of highly inactive nearly doubled in 18$24-y$-olds and is now similar to other age groups. Data in each of the five Nordic countries showed that the proportions of inactive and highly inactive increased from 2011 to 2014 in $18-24-y$-olds in Iceland and Norway (Iceland inactive: from $21.6 \%$ to $32.8 \%$ and Norway inactive: from $22.6 \%$ to $45.3 \%$ ).

Finally, the proportion of highly active increased in $25-44-y$-olds in Norway.

\section{Sedentary behaviour}

In 2014, the proportion with high recreational screen time was higher in 18-24-y-olds than in 25-44- and 45-65-y-olds in the Nordic region. Similar results were found in Denmark, Finland and Norway. In Sweden and Iceland, an inverse age gradient was found, i.e. the proportion with high recreational screen time decreased across age groups.

Data from 2011 to 2014 showed no change in mean recreational screen time in the three age groups in the Nordic region, but the pattern of screen time changed because TV time decreased in 18-24- and 25-44-y-olds and computer time increased in 25-44- and 45-65-y-olds (Table 44). Mixed results were found for the development of recreational screen time from 2011 to 2014 in 18-24- and 25-44-y-olds in the five Nordic countries, but not in $45-65$-y-olds where an increase was found in Denmark, Finland and Iceland.

Table 44: Mean and proportion $(95 \% \mathrm{Cl}$ ) for estimates of sedentary behaviour among adults according to age in the Nordic region. NORMO 2011 and 2014

\begin{tabular}{|c|c|c|c|c|c|c|}
\hline & \multicolumn{6}{|c|}{ Nordic region@ } \\
\hline & \multicolumn{2}{|c|}{$18-24 \mathrm{Y}$} & \multicolumn{2}{|c|}{$25-44 Y$} & \multicolumn{2}{|c|}{$45-65 y$} \\
\hline & $\begin{array}{r}2011 \\
(n=989)\end{array}$ & $\begin{array}{r}2014 \\
(n=793)\end{array}$ & $\begin{array}{r}2011 \\
(n=3,307)\end{array}$ & $\begin{array}{r}2014 \\
(n=2,917)\end{array}$ & $\begin{array}{r}2011 \\
(n=4,577)\end{array}$ & $\begin{array}{r}2014 \\
(n=4,382)\end{array}$ \\
\hline $\begin{array}{l}\text { Recreational screen } \\
\text { time }(\mathrm{h} / \mathrm{d})\end{array}$ & $\begin{array}{r}5.0 \\
(4.8 ; 5.2)\end{array}$ & $\begin{array}{r}4.7 \\
(4.5 ; 5.0)\end{array}$ & $\begin{array}{r}3.8 \\
(3.7 ; 3.9)\end{array}$ & $\begin{array}{r}3.8 \\
(3.7 ; 3.9)\end{array}$ & $\begin{array}{r}3.6 \\
(3.6 ; 3.7)\end{array}$ & $\begin{array}{r}3.7 \\
(3.7 ; 3.8)\end{array}$ \\
\hline $\mathrm{TV}(\mathrm{h} / \mathrm{d})$ & $\begin{array}{r}2.2 \\
(2.1 ; 2.3)\end{array}$ & $\begin{array}{r}1.9^{* *} \\
(1.8 ; 2.0)\end{array}$ & $\begin{array}{r}2.2 \\
(2.2 ; 2.3)\end{array}$ & $\begin{array}{r}2.0^{* * *} \\
(1.9 ; 2.1)\end{array}$ & $\begin{array}{r}2.5 \\
(2.4 ; 2.6)\end{array}$ & $\begin{array}{r}2.5 \\
(2.5 ; 2.6)\end{array}$ \\
\hline Computer (h/d) & $\begin{array}{r}2.8 \\
(2.6 ; 2.9)\end{array}$ & $\begin{array}{r}3.0 \\
(2.8 ; 3.1)\end{array}$ & $\begin{array}{r}1.5 \\
(1.5 ; 1.6)\end{array}$ & $\begin{array}{r}1.9^{* * *} \\
(1.8 ; 1.9)\end{array}$ & $\begin{array}{r}1.1 \\
(1.1 ; 1.2)\end{array}$ & $\begin{array}{r}1.4^{* * *} \\
(1.3 ; 1.4)\end{array}$ \\
\hline $\begin{array}{l}\text { High recreational } \\
\text { screen time }(>4 \mathrm{~h} / \mathrm{d}) \\
(\%)\end{array}$ & $\begin{array}{r}48.3 \\
(45.2 ; 51.3)\end{array}$ & $\begin{array}{r}46.5 \\
(43.2 ; 49.9)\end{array}$ & $\begin{array}{r}26.3 \\
(24.8 ; 27.8)\end{array}$ & $\begin{array}{r}27.5 \\
(25.8 ; 29.1)\end{array}$ & $\begin{array}{r}24.5 \\
(23.2 ; 25.7)\end{array}$ & $\begin{array}{r}27.1^{* \star} \\
(25.8 ; 28.4)\end{array}$ \\
\hline
\end{tabular}

Note: $\quad{ }^{* *} p<0.01,{ }^{* * *} p<0.001$ : Differences between survey years 2011 and 2014 using t-tests and Chi-square tests.

@Weighted according to population size in the five Nordic countries. 
Between 2011 and 2014, there was an increase in the proportion with high recreational screen time in $45-65$-y-olds in the Nordic region. Moreover, the proportion with high recreational screen time increased in $18-24-y$-olds in Finland (from $37.6 \%$ to $48.2 \%$ ) and decreased in Norway (from $65.1 \%$ to $49.6 \%$ ). In $25-44$-y-olds, an increase was found in Finland (from $27.7 \%$ to $33.8 \%$ ) and Iceland (from $20.6 \%$ to $27.7 \%$ ), and in $45-65$-y-olds, the proportion with high recreational screen time increased in Denmark (from $27.4 \%$ to $31.4 \%$ ) and Finland (from $23.7 \%$ to $32.8 \%$ ). Thus, Finland was the only Nordic country where an increase in the proportion with high recreational screen time was found in all age groups.

\subsubsection{Education}

\section{Physical activity}

In 2014, the proportion of inactive was higher among the low and medium educated than among the high educated in the Nordic region. In Iceland and Norway, the proportion of inactive was higher among the low educated than among the high educated, while in Denmark and Finland the proportion of inactive was higher among the medium educated than among the high educated. No difference between education groups was found in Sweden. Large social differences in physical activity (inactive) were found among adults in the Nordic Region and in Sweden, Iceland and Norway while moderate differences were seen in Denmark and Finland.

Analyses showed an increase in mean MVPA from 2011 to 2014 among the high educated in the Nordic region (Table 45). Mean MVPA increased among the high educated in all the Nordic countries, except in Finland.

Data showed that the proportion of highly inactive increased from 2011 to 2014 among the medium and high educated in the Nordic region. No change was found in the proportion of inactive between 2011 and 2014 in the three education groups in the Nordic region. When analysing each of the five Nordic countries, the proportion of inactive decreased from 2011 to 2014 among the low educated in Finland (from 37.9\% to 29.3\%) while no change was observed among the medium and high educated in the five Nordic countries. Moreover, between 2011 and 2014, the proportion of highly inactive increased among the low educated in Iceland, decreased among the low educated in Finland, and increased among the medium educated in Norway. Thus, findings were not systematic in the Nordic countries. 
Table 45: Mean and proportion $(95 \% \mathrm{Cl})$ for estimates of physical activity among adults according to education in the Nordic region. NORMO 2011 and 2014

\begin{tabular}{|c|c|c|c|c|c|c|}
\hline & \multicolumn{6}{|c|}{ Nordic region@ } \\
\hline & \multicolumn{2}{|c|}{ Low } & \multicolumn{2}{|c|}{ Medium } & \multicolumn{2}{|c|}{ High } \\
\hline & $\begin{array}{r}2011 \\
(n=1,337)\end{array}$ & $\begin{array}{r}2014 \\
(n=1,001)\end{array}$ & $\begin{array}{r}2011 \\
(n=3,703)\end{array}$ & $\begin{array}{r}2014 \\
(n=2,918)\end{array}$ & $\begin{array}{r}2011 \\
(n=3,481)\end{array}$ & $\begin{array}{r}2014 \\
(n=4,098)\end{array}$ \\
\hline MVPA (h/wk) & $\begin{array}{r}5.2 \\
(4 \cdot 9 ; 5 \cdot 5)\end{array}$ & $\begin{array}{r}5.0 \\
(4.8 ; 5 \cdot 3)\end{array}$ & $\begin{array}{r}4.9 \\
(4.8 ; 5.1)\end{array}$ & $\begin{array}{r}5.0 \\
(4.8 ; 5.1)\end{array}$ & $\begin{array}{r}4.6 \\
(4 \cdot 4 ; 4 \cdot 7)\end{array}$ & $\begin{array}{r}5 \cdot 0^{* \star \star \star} \\
(4.8 ; 5 \cdot 1)\end{array}$ \\
\hline MPA (h/wk) & $\begin{array}{r}3.5 \\
(3.3 ; 3.7)\end{array}$ & $\begin{array}{r}3.2^{-} \\
(2.9 ; 3.4)\end{array}$ & $\begin{array}{r}3.2 \\
(3.0 ; 3.3)\end{array}$ & $\begin{array}{r}3.2 \\
(3.0 ; 3.3)\end{array}$ & $\begin{array}{r}2.9 \\
(2.8 ; 3.0)\end{array}$ & $\begin{array}{r}3.3^{* * *} \\
(3.2 ; 3.4)\end{array}$ \\
\hline VPA (h/wk) & $\begin{array}{r}1.6 \\
(1.5 ; 1.7)\end{array}$ & $\begin{array}{r}1.9^{* *} \\
(1.7 ; 2.0)\end{array}$ & $\begin{array}{r}1.8 \\
(1.7 ; 1.8)\end{array}$ & $\begin{array}{r}1.8 \\
(1.7 ; 1.8)\end{array}$ & $\begin{array}{r}1.7 \\
(1.6 ; 1.8)\end{array}$ & $\begin{array}{r}1.7 \\
(1.7 ; 1.8)\end{array}$ \\
\hline Inactive(\%) & $\begin{array}{r}38.0 \\
(35.0 ; 40.9)\end{array}$ & $\begin{array}{r}36.6 \\
(33.1 ; 40.1)\end{array}$ & $\begin{array}{r}33.4 \\
(31.9 ; 34.9)\end{array}$ & $\begin{array}{r}33.7 \\
(32.0 ; 35.4)\end{array}$ & $\begin{array}{r}30.1 \\
(28.7 ; 31.6)\end{array}$ & $\begin{array}{r}30.1 \\
(28.7 ; 31.4)\end{array}$ \\
\hline Highly inactive ${ }^{\#}(\%)$ & $\begin{array}{r}14.2 \\
(12.1 ; 16.3)\end{array}$ & $\begin{array}{r}14.7 \\
(12.1 ; 17 \cdot 3)\end{array}$ & $\begin{array}{r}10.6 \\
(9.6 ; 11.6)\end{array}$ & $\begin{array}{r}12.9^{* *} \\
(11.7 ; 14.1)\end{array}$ & $\begin{array}{r}7.8 \\
(6.9 ; 8.7)\end{array}$ & $\begin{array}{r}9.0^{*} \\
(8.2 ; 9.9)\end{array}$ \\
\hline Active(\%) & $\begin{array}{r}62.0 \\
(59.1 ; 65.0)\end{array}$ & $\begin{array}{r}63.4 \\
(59.9 ; 66.9)\end{array}$ & $\begin{array}{r}66.6 \\
(65.1 ; 68.1)\end{array}$ & $\begin{array}{r}66.3 \\
(64.6 ; 68.0)\end{array}$ & $\begin{array}{r}69.9 \\
(68.4 ; 71.3)\end{array}$ & $\begin{array}{r}69.9 \\
(68.6 ; 71.3)\end{array}$ \\
\hline Highly actives $(\%)$ & $\begin{array}{r}12.9 \\
(10.8 ; 14.9)\end{array}$ & $\begin{array}{r}14.4 \\
(11.8 ; 16.9)\end{array}$ & $\begin{array}{r}14.2 \\
(13.0 ; 15 \cdot 3)\end{array}$ & $\begin{array}{r}14.8 \\
(13.5 ; 16.1)\end{array}$ & $\begin{array}{r}12.4 \\
(11.4 ; 13.5)\end{array}$ & $\begin{array}{r}15.0^{* * *} \\
(13.9 ; 16.1)\end{array}$ \\
\hline $\begin{array}{l}\text { Relative difference } \\
\text { inactive" (\%) }\end{array}$ & - & - & - & - & -26 & -22 \\
\hline
\end{tabular}

Note: $\quad-p<0.10,{ }^{*} p<0.05,{ }^{* *} p<0.01,{ }^{* * *} p<0.001$ : Differences between survey years 2011 and 2014 using ttests and Chi-square tests.

@Weighted according to population size in the five Nordic countries.

\#Subgroup of inactive that do not add up to $100 \%$.

"Subgroup of active that do not add up to $100 \%$.

"Relative difference (\%) = [(value High education group - value Low education group $) /$ value High education group] ${ }^{*} 100$.

The proportion of highly active increased among the high educated in the Nordic region and a systematic upward tendency was found in all the Nordic countries, except in Finland. However, the increase was only significant in Denmark.

Between 2011 and 2014, the social difference in physical activity did not change among adults in the Nordic region (Figure 5). Data in each of the five Nordic countries showed mixed results because the social difference increased slightly in Sweden, decreased in Denmark and Finland while no change was found in Iceland and Norway. 
Figure 5: The development from 2011 to 2014 in the social difference in physical activity (inactive) among adults in the Nordic region. NORMO 2011 and 2014

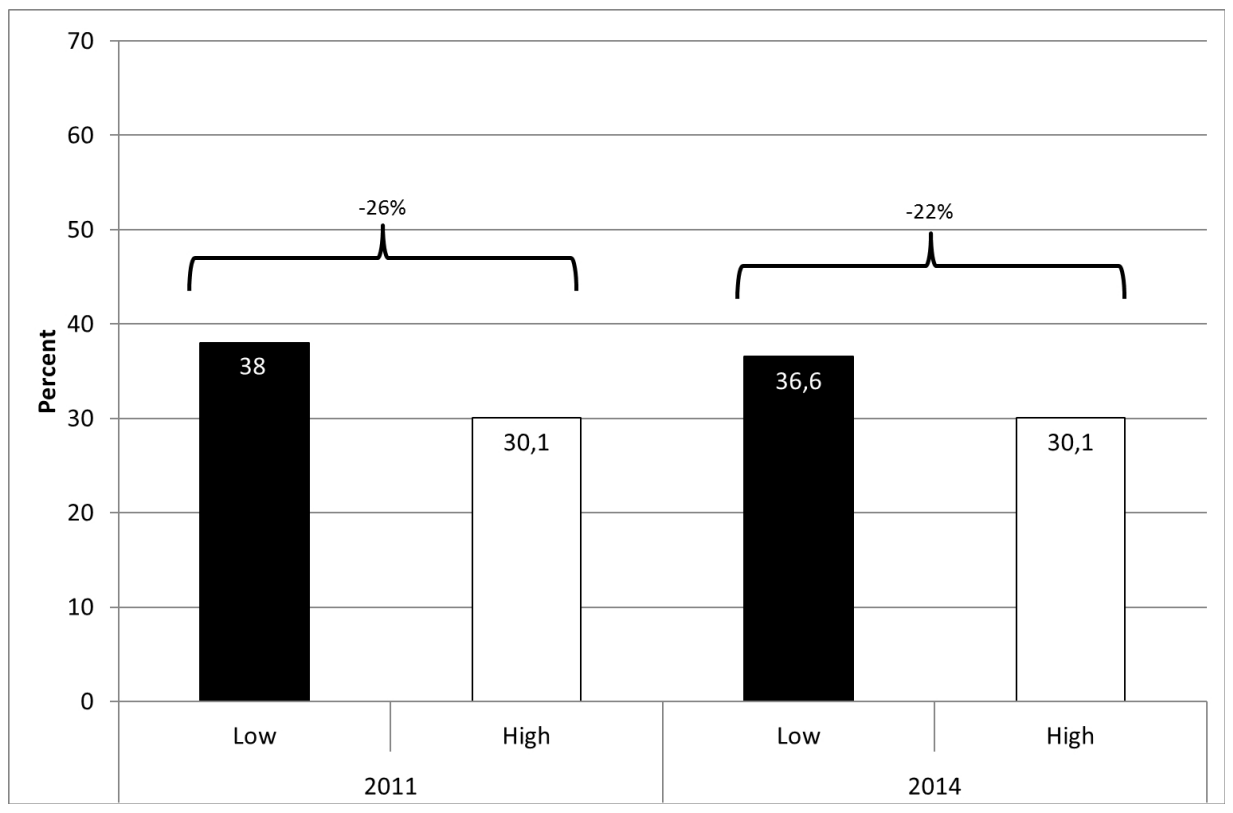

\section{Sedentary behaviour}

In 2014, an inverse social gradient was found in sedentary behaviour among adults in the Nordic region and in Denmark and Sweden i.e. the higher the education level, the lower the proportion with high recreational screen time. In Norway, the proportion with high recreational screen time was higher among the low educated than among the medium and high educated while in Iceland the proportion was higher among the low and medium educated than among the high educated. In Finland, the proportion with high recreational screen time was higher among the medium educated than among the high educated. Large social differences were found in sedentary behaviour (high recreational screen time) among adults in the Nordic region and in each of the five Nordic countries, except in Finland with just a small difference.

Data from 2011 to 2014 showed an increase in mean computer time among the low educated in the Nordic region that also explained the increase in mean recreational screen time (Table 46). Mean recreational screen time did not change among the medium and high educated, but the pattern of screen time changed from less TV time to more computer time. Between 2011 and 2014, data in each of the five Nordic countries 
showed an increase in mean recreational screen time among the low educated in Denmark and Iceland due to more computer time while results among the medium and high educated were mixed.

In the Nordic region, an increase from 2011 to 2014 was found in the proportion with high recreational screen time among the low educated. Between 2011 and 2014, data in each of the five Nordic countries showed that the proportion with high recreational screen time increased among the low educated in Denmark (from $33.7 \%$ to 44.6\%), Sweden (from $26.1 \%$ to $37.7 \%$ ) and Iceland (from $24.9 \%$ to $30.6 \%$ ). An increase was also found in the proportion with high recreational screen time among the medium educated in Finland (from $28.1 \%$ to $38.5 \%$ ) while a decrease was seen in Norway (from $36.4 \%$ to $28.3 \%$ ). Finally among the high educated the proportion with high recreational screen time increased in Finland (from $24.4 \%$ to $31.3 \%$ ) and decreased in Norway (from $31.9 \%$ to $25.9 \%$ ). Hence, the proportion with high recreational screen time increased among the low educated in Denmark, Sweden and Iceland while results among the medium and high educated were mixed.

Table 46: Mean and proportion $(95 \% \mathrm{Cl}$ ) for estimates of sedentary behaviour among adults according to education in the Nordic region. NORMO 2011 and 2014

\begin{tabular}{|c|c|c|c|c|c|c|}
\hline & \multicolumn{6}{|c|}{ Nordic region@ } \\
\hline & \multicolumn{2}{|c|}{ Low } & \multicolumn{2}{|c|}{ Medium } & \multicolumn{2}{|c|}{ High } \\
\hline & $\begin{array}{r}2011 \\
(n=1,392)\end{array}$ & $\begin{array}{r}2014 \\
(n=971)\end{array}$ & $\begin{array}{r}2011 \\
(n=3,858)\end{array}$ & $\begin{array}{r}2014 \\
(n=2,956)\end{array}$ & $\begin{array}{r}2011 \\
(n=3,583)\end{array}$ & $\begin{array}{r}2014 \\
(n=4,151)\end{array}$ \\
\hline $\begin{array}{l}\text { Recreational screen } \\
\text { time }(\mathrm{h} / \mathrm{d})\end{array}$ & $\begin{array}{r}4.1 \\
(3.9 ; 4 \cdot 3)\end{array}$ & $\begin{array}{r}4 \cdot 5^{* *} \\
(4 \cdot 3 ; 4 \cdot 7)\end{array}$ & $\begin{array}{r}3.9 \\
(3.8 ; 4.0)\end{array}$ & $\begin{array}{r}3.8 \\
(3.7 ; 3.9)\end{array}$ & $\begin{array}{r}3.7 \\
(3.6 ; 3.8)\end{array}$ & $\begin{array}{r}3.6 \\
(3.5 ; 3.7)\end{array}$ \\
\hline $\mathrm{TV}(\mathrm{h} / \mathrm{d})$ & $\begin{array}{r}2.7 \\
(2.5 ; 2.8)\end{array}$ & $\begin{array}{r}2.7 \\
(2.6 ; 2.9)\end{array}$ & $\begin{array}{r}2.4 \\
(2.3 ; 2.4)\end{array}$ & $\begin{array}{r}2.2^{* *} \\
(2.1 ; 2.3)\end{array}$ & $\begin{array}{r}2.1 \\
(2.1 ; 2.2)\end{array}$ & $\begin{array}{r}1.9^{* * *} \\
(1.9 ; 2.0)\end{array}$ \\
\hline Computer (h/d) & $\begin{array}{r}1.4 \\
(1.3 ; 1.5)\end{array}$ & $\begin{array}{r}1.9^{* * *} \\
(1.7 ; 2.0)\end{array}$ & $\begin{array}{r}1.6 \\
(1.5 ; 1.6)\end{array}$ & $\begin{array}{r}1.7^{* * *} \\
(1.7 ; 1.8)\end{array}$ & $\begin{array}{r}1.6 \\
(1.5 ; 1.6)\end{array}$ & $\begin{array}{r}1.8^{* * *} \\
(1.7 ; 1.9)\end{array}$ \\
\hline $\begin{array}{l}\text { High recreational screen } \\
\text { time }(>4 \mathrm{~h} / \mathrm{d})(\%)\end{array}$ & $\begin{array}{r}31.8 \\
(29.1 ; 34.5)\end{array}$ & $\begin{array}{r}39 \cdot 2^{* *} \\
(35.6 ; 42.8)\end{array}$ & $\begin{array}{r}29.2 \\
(27.8 ; 30.6)\end{array}$ & $\begin{array}{r}29.8 \\
(28.1 ; 31.4)\end{array}$ & $\begin{array}{r}25.8 \\
(24.4 ; 27.1)\end{array}$ & $\begin{array}{r}24.4 \\
(23.2 ; 25.7)\end{array}$ \\
\hline $\begin{array}{l}\text { Relative difference } \\
\text { high recreational } \\
\text { screen time" (\%) }\end{array}$ & - & - & - & - & -23 & -60 \\
\hline
\end{tabular}

Note: $\quad{ }^{* *} p<0.01,{ }^{* * *} p<0.001$ : Differences between survey years 2011 and 2014 using t-tests and Chi-square tests.

@Weighted according to population size in the five Nordic countries.

"Relative difference $(\%)=$ [(value High education group - value Low education group)/value High education group]*100. 
In the Nordic region, the social difference in sedentary behaviour increased among adults from 2011 to 2014 because the low educated increased their recreational screen time. The social difference in sedentary behaviour increased in all the Nordic countries, except in Finland where a decrease was seen.

\subsubsection{Children}

\section{Physical activity}

In 2014, children in the Nordic region spent about six and a half hours weekly on MVPA during leisure time which is close to the total amount for meeting the physical activity recommendations. Results showed that six in ten children were physically inactive in the Nordic region. Ranking of the proportions of inactive and with high recreational screen time in the five Nordic countries is shown in Table 47. The proportion of inactive was lower in Finland than in the other Nordic countries and higher in Sweden than in the other Nordic countries. Moreover, the proportion of inactive was higher in Denmark and Norway than in Iceland.

Table 47: Ranking from lowest to highest proportions of inactive and with high recreational screen time among children in the five Nordic countries. NORMO 2014

\begin{tabular}{lclc} 
& Inactive (\%) & & High recreational screen time $(>4 \mathrm{~h} /$ day $)(\%)$ \\
Finland & 41.4 & Iceland & 5.5 \\
Iceland & 47.7 & Sweden & 13.3 \\
Denmark & 59.2 & Finland & 14.5 \\
Norway & 59.8 & Norway & 15.9 \\
Sweden & 68.9 & Denmark & 20.1 \\
\hline
\end{tabular}

Table 48 shows the development of physical activity from 2011 to 2014 among children in the Nordic countries. Mean MVPA and the proportions of inactive and highly inactive did not change from 2011 to 2014 in the Nordic region. Few changes were found in each of the five Nordic countries between 2011 and 2014. Mean MVPA decreased in Denmark, however no change was detected in the proportion of inactive. In Norway, the proportions of inactive and highly inactive decreased. 
Table 48: Mean and proportion $(95 \% \mathrm{Cl})$ for estimates of physical activity among children in the Nordic countries. NORMO 2011 and 2014

\begin{tabular}{|c|c|c|c|c|c|c|c|c|c|c|c|c|}
\hline & \multicolumn{2}{|c|}{ Denmark } & \multicolumn{2}{|c|}{ Finland } & \multicolumn{2}{|c|}{ Sweden } & \multicolumn{2}{|c|}{ Iceland } & \multicolumn{2}{|c|}{ Norway } & \multicolumn{2}{|c|}{ Nordic region@ } \\
\hline & $\begin{array}{r}2011 \\
(\mathrm{n}=606)\end{array}$ & $\begin{array}{r}2014 \\
(n=596)\end{array}$ & $\begin{array}{r}2011 \\
(n=495)\end{array}$ & $\begin{array}{r}2014 \\
(n=500)\end{array}$ & $\begin{array}{r}2011 \\
(n=499)\end{array}$ & $\begin{array}{r}2014 \\
(n=498)\end{array}$ & $\begin{array}{r}2011 \\
(n=510)\end{array}$ & $\begin{array}{r}2014 \\
(n=510)\end{array}$ & $\begin{array}{r}2011 \\
(n=353)\end{array}$ & $\begin{array}{r}2014 \\
(n=358)\end{array}$ & $\begin{array}{r}2011 \\
(n=2,463)\end{array}$ & $\begin{array}{r}2014 \\
(n=2,462)\end{array}$ \\
\hline MVPA (h/wk) & $\begin{array}{r}7.0 \\
(6.6 ; 7.4)\end{array}$ & $\begin{array}{r}6.4^{*} \\
(6.1 ; 6.7)\end{array}$ & $\begin{array}{r}8.5 \\
(8.0 ; 9.0)\end{array}$ & $\begin{array}{r}8.4 \\
(7.9 ; 8.8)\end{array}$ & $\begin{array}{r}5.6 \\
(5.2 ; 6.0)\end{array}$ & $\begin{array}{r}5.6 \\
(5.2 ; 6.0)\end{array}$ & $\begin{array}{r}7.5 \\
(7.1 ; 7.9)\end{array}$ & $\begin{array}{r}7.5 \\
(7.1 ; 7.9)\end{array}$ & $\begin{array}{r}5.7 \\
(5.3 ; 6.2)\end{array}$ & $\begin{array}{r}6.2 \\
(5.7 ; 6.6)\end{array}$ & $\begin{array}{r}6.6 \\
(6.4 ; 6.8)\end{array}$ & $\begin{array}{r}6.5 \\
(6.3 ; 6.6)\end{array}$ \\
\hline Inactive(\%) & $\begin{array}{r}55.6 \\
(51.7 ; 59.6)\end{array}$ & $\begin{array}{r}59.2 \\
(55.2 ; 63.1)\end{array}$ & $\begin{array}{r}40.3 \\
(36.0 ; 44.6)\end{array}$ & $\begin{array}{r}41.4 \\
(37.1 ; 45.7)\end{array}$ & $\begin{array}{r}71.0 \\
(67.0 ; 74.9)\end{array}$ & $\begin{array}{r}68.9 \\
(64.8 ; 72.9)\end{array}$ & $\begin{array}{r}49.1 \\
(44.8 ; 53.4)\end{array}$ & $\begin{array}{r}47.7 \\
(43.4 ; 52.0)\end{array}$ & $\begin{array}{r}67.7 \\
(62.8 ; 72.6)\end{array}$ & $\begin{array}{r}59.8^{*} \\
(54.7 ; 64.8)\end{array}$ & $\begin{array}{r}60.3 \\
(58.4 ; 62.2)\end{array}$ & $\begin{array}{r}59.1 \\
(57.1 ; 61.0)\end{array}$ \\
\hline Highly inactive" (\%) & $\begin{array}{r}2.0 \\
(0.9 ; 3.1)\end{array}$ & $\begin{array}{r}2.2 \\
(1.0 ; 3.4)\end{array}$ & $\begin{array}{r}0.9 \\
(0.1 ; 1.7)\end{array}$ & $\begin{array}{r}0.8 \\
(0.0 ; 1.7)\end{array}$ & $\begin{array}{r}5.6 \\
(3.6 ; 7.7)\end{array}$ & $\begin{array}{r}4.1 \\
(2.4: 5.9)\end{array}$ & $\begin{array}{r}1.6 \\
(0.5 ; 2.7)\end{array}$ & $\begin{array}{r}2.7 \\
(1.3 ; 4.1)\end{array}$ & $\begin{array}{r}3.6 \\
(1.7 ; 5.5)\end{array}$ & $\begin{array}{r}1.3^{*} \\
(0.1 ; 2.4)\end{array}$ & $\begin{array}{r}3.4 \\
(2.6 ; 4 \cdot 1)\end{array}$ & $\begin{array}{r}2.4^{-} \\
(1.8 ; 3.1)\end{array}$ \\
\hline Active(\%) & $\begin{array}{r}44.4 \\
(40.4 ; 48.3)\end{array}$ & $\begin{array}{r}40.8 \\
(36.9 ; 44.8)\end{array}$ & $\begin{array}{r}59.7 \\
(55.4 ; 64.0)\end{array}$ & $\begin{array}{r}58.6 \\
(54.3 ; 62.9)\end{array}$ & $\begin{array}{r}29.0 \\
(25.1 ; 33.0)\end{array}$ & $\begin{array}{r}31.1 \\
(27.1 ; 35.2)\end{array}$ & $\begin{array}{r}50.9 \\
(46.6 ; 55.2)\end{array}$ & $\begin{array}{r}52.3 \\
(48.0 ; 56.6)\end{array}$ & $\begin{array}{r}32.3 \\
(27.4 ; 37.2)\end{array}$ & $\begin{array}{r}40.2^{*} \\
(35.2 ; 45.3)\end{array}$ & $\begin{array}{r}39.7 \\
(37.8 ; 41.6)\end{array}$ & $\begin{array}{r}40.9 \\
(39.0 ; 42.9)\end{array}$ \\
\hline
\end{tabular}

Note: $\quad-p<0.10,{ }^{*} p<0.05$ : Differences between survey years 2011 and 2014 using t-tests and Chi-square tests.

\#Subgroup of inactive that do not add up to $100 \%$.

@Weighted according to population size in the five Nordic countries. 


\section{Sedentary behaviour}

In 2014, children in the Nordic region spent on average three hours daily on TV and/or computer time during leisure. More than one in seven children spent more than four hours per day on recreational screen time in the Nordic region. The proportion with high recreational screen time was lower in Iceland than in the other Nordic countries and higher in Denmark than in Finland and Sweden (Table 47).

Between 2011 and 2014, mean recreational screen time decreased in the Nordic region while mixed results were found in the Nordic countries (Table 49). The pattern of recreational screen time changed in children from 2011 to 2014 in the same way as in adults, i.e. less TV time and more computer time probably due to smart phones and tablets have become more popular and replaced TV. No change was observed between 2011 and 2014 in the proportion with high recreational screen time in the Nordic region and in each of the five Nordic countries. 
Table 49: Mean and proportion ( $95 \% \mathrm{Cl})$ for estimates of sedentary behaviour among children in the Nordic countries. NORMO 2011 and 2014

\begin{tabular}{|c|c|c|c|c|c|c|c|c|c|c|c|c|}
\hline & \multicolumn{2}{|c|}{ Denmark } & \multicolumn{2}{|c|}{ Finland } & \multicolumn{2}{|c|}{ Sweden } & \multicolumn{2}{|c|}{ Iceland } & \multicolumn{2}{|c|}{ Norway } & \multicolumn{2}{|c|}{ Nordic region@ } \\
\hline & $\begin{array}{r}2011 \\
(n=599)\end{array}$ & $\begin{array}{r}2014 \\
(n=588)\end{array}$ & $\begin{array}{r}2011 \\
(n=488)\end{array}$ & $\begin{array}{r}2014 \\
(n=490)\end{array}$ & $\begin{array}{r}2011 \\
(n=478)\end{array}$ & $\begin{array}{r}2014 \\
(n=492)\end{array}$ & $\begin{array}{r}2011 \\
(n=504)\end{array}$ & $\begin{array}{r}2014 \\
(n=508)\end{array}$ & $\begin{array}{r}2011 \\
(n=332)\end{array}$ & $\begin{array}{r}2014 \\
(n=341)\end{array}$ & $\begin{array}{r}2011 \\
(n=2,401)\end{array}$ & $\begin{array}{r}2014 \\
(n=2,419)\end{array}$ \\
\hline $\begin{array}{l}\text { Recreational } \\
\text { screen time }(\mathrm{h} / \mathrm{d})\end{array}$ & $\begin{array}{r}3.1 \\
(2.9 ; 3.3)\end{array}$ & $\begin{array}{r}3.4^{*} \\
(3.2 ; 3.6)\end{array}$ & $\begin{array}{r}3.1 \\
(2.8 ; 3 \cdot 3)\end{array}$ & $\begin{array}{r}2.9 \\
(2.6 ; 3.1)\end{array}$ & $\begin{array}{r}3.1 \\
(2.9 ; 3 \cdot 3)\end{array}$ & $\begin{array}{r}2.8^{* *} \\
(2.6 ; 2.9)\end{array}$ & $\begin{array}{r}2.4 \\
(2.2 ; 2.6)\end{array}$ & $\begin{array}{r}2.2^{-} \\
(2.0 ; 2.3)\end{array}$ & $\begin{array}{r}3.4 \\
(3.1 ; 3.7)\end{array}$ & $\begin{array}{r}3.1 \\
(2.8 ; 3 \cdot 4)\end{array}$ & $\begin{array}{r}3.2 \\
(3.1 ; 3 \cdot 3)\end{array}$ & $\begin{array}{r}3.0^{*} \\
(2.9 ; 3.1)\end{array}$ \\
\hline $\operatorname{TV}(\mathrm{h} / \mathrm{d})$ & $\begin{array}{r}1.9 \\
(1.8 ; 2.0)\end{array}$ & $\begin{array}{r}1.8 \\
(1.7 ; 2.0)\end{array}$ & $\begin{array}{r}2.1 \\
(1.9 ; 2.3)\end{array}$ & $\begin{array}{r}1.5^{* * *} \\
(1.4 ; 1.7)\end{array}$ & $\begin{array}{r}2.0 \\
(1.8 ; 2.1)\end{array}$ & $\begin{array}{r}1.5^{* * *} \\
(1.4 ; 1.6)\end{array}$ & $\begin{array}{r}1.4 \\
(1.3 ; 1.5)\end{array}$ & $\begin{array}{r}1.2^{* *} \\
(1.1 ; 1.3)\end{array}$ & $\begin{array}{r}2.1 \\
(1.9 ; 2.3)\end{array}$ & $\begin{array}{r}1.7^{* *} \\
(1.5 ; 1.9)\end{array}$ & $\begin{array}{r}2.0 \\
(1.9 ; 2.1)\end{array}$ & $\begin{array}{r}1.6^{* * *} \\
(1.5 ; 1.7)\end{array}$ \\
\hline Computer (h/d) & $\begin{array}{r}1.2 \\
(1.1 ; 1.3)\end{array}$ & $\begin{array}{r}1.6^{* * *} \\
(1.5 ; 1.7)\end{array}$ & $\begin{array}{r}1.0 \\
(0.9 ; 1.1)\end{array}$ & $\begin{array}{r}1.3^{* * *} \\
(1.2 ; 1.5)\end{array}$ & $\begin{array}{r}1.2 \\
(1.1 ; 1.4)\end{array}$ & $\begin{array}{r}1.3 \\
(1.2 ; 1.4)\end{array}$ & $\begin{array}{r}1.0 \\
(0.9 ; 1.1)\end{array}$ & $\begin{array}{r}1.0 \\
(0.9 ; 1.1)\end{array}$ & $\begin{array}{r}1.4 \\
(1.2 ; 1.5)\end{array}$ & $\begin{array}{r}1.5 \\
(1.3 ; 1.8)\end{array}$ & $\begin{array}{r}1.2 \\
(1.2 ; 1.3)\end{array}$ & $\begin{array}{r}1.4^{* * *} \\
(1.4 ; 1.5)\end{array}$ \\
\hline $\begin{array}{l}\text { High recreational } \\
\text { screen time (>4 } \\
\text { h/d) (\%) }\end{array}$ & $\begin{array}{r}16.7 \\
(13.7 ; 19.7)\end{array}$ & $\begin{array}{r}20.1 \\
(16.8 ; 23.3)\end{array}$ & $\begin{array}{r}16.3 \\
(13.0 ; 19.6)\end{array}$ & $\begin{array}{r}14.5 \\
(11.4 ; 17.7)\end{array}$ & $\begin{array}{r}16.3 \\
(13.0 ; 19.7)\end{array}$ & $\begin{array}{r}13.3 \\
(10.3 ; 16.3)\end{array}$ & $\begin{array}{r}8.5 \\
(6.1 ; 10.9)\end{array}$ & $\begin{array}{r}5.5^{-} \\
(3.5 ; 7.5)\end{array}$ & $\begin{array}{r}21.2 \\
(16.8 ; 25.6)\end{array}$ & $\begin{array}{r}15.9^{-} \\
(11.9 ; 19.9)\end{array}$ & $\begin{array}{r}17.3 \\
(15.8 ; 18.8)\end{array}$ & $\begin{array}{r}15.5^{-} \\
(14.0 ; 16.9)\end{array}$ \\
\hline
\end{tabular}

Note: $\quad-p<0.10,{ }^{*} p<0.05,{ }^{* *} p<0.01,{ }^{* * *} p<0.001$ : Differences between survey years 2011 and 2014 using t-tests and Chi-square tests.

@Weighted according to population size in the five Nordic countries. 


\subsubsection{Gender}

\section{Physical activity}

In 2014, the proportion of inactive was higher in girls than in boys in the Nordic region $(64.2 \%$ vs. $54.0 \%)$. A higher proportion of inactive girls was also seen in Denmark, Finland and Norway while no significant gender difference was observed in Sweden and Iceland.

Data from 2011 to 2014 showed a decrease in the proportion of highly inactive girls in the Nordic region while no change was found in boys (Table 50 and 51). Mean MVPA and the proportion of inactive did not change from 2011 to 2014 in boys and girls. When analysing each of the five Nordic countries, mean MVPA decreased in girls in Denmark, but no significant change was detected in the proportion of inactive. Furthermore, a decrease was found in the proportion of highly inactive in girls in Norway whereas an increase was found in girls in Iceland. Thus, significant findings in the development of physical activity were few and not systematic in the Nordic countries. 
Table 50: Mean and proportion ( $95 \% \mathrm{Cl}$ ) for estimates of physical activity among boys in the Nordic countries. NORMO 2011 and 2014

\begin{tabular}{|c|c|c|c|c|c|c|c|c|c|c|c|c|}
\hline & \multicolumn{2}{|c|}{ Denmark } & \multicolumn{2}{|c|}{ Finland } & \multicolumn{2}{|c|}{ Sweden } & \multicolumn{2}{|c|}{ Iceland } & \multicolumn{2}{|c|}{ Norway } & \multicolumn{2}{|c|}{ Nordic region@ } \\
\hline & $\begin{array}{r}2011 \\
(n=318)\end{array}$ & $\begin{array}{r}2014 \\
(n=294)\end{array}$ & $\begin{array}{r}2011 \\
(n=242)\end{array}$ & $\begin{array}{r}2014 \\
(n=243)\end{array}$ & $\begin{array}{r}2011 \\
(n=272)\end{array}$ & $\begin{array}{r}2014 \\
(n=257)\end{array}$ & $\begin{array}{r}2011 \\
(n=244)\end{array}$ & $\begin{array}{r}2014 \\
(n=255)\end{array}$ & $\begin{array}{r}2011 \\
(n=169)\end{array}$ & $\begin{array}{r}2014 \\
(n=173)\end{array}$ & $\begin{array}{r}2011 \\
(n=1,245)\end{array}$ & $\begin{array}{r}2014 \\
(n=1,222)\end{array}$ \\
\hline MVPA (h/wk) & $\begin{array}{r}7.2 \\
(6.7 ; 7.8)\end{array}$ & $\begin{array}{r}7.0 \\
(6.6 ; 7.5)\end{array}$ & $\begin{array}{r}9.9 \\
(9.3 ; 10.6)\end{array}$ & $\begin{array}{r}9.2 \\
(8.5 ; 9.8)\end{array}$ & $\begin{array}{r}6.3 \\
(5.7 ; 6.9)\end{array}$ & $\begin{array}{r}6.0 \\
(5.4 ; 6.5)\end{array}$ & $\begin{array}{r}7.8 \\
(7.2 ; 8.4)\end{array}$ & $\begin{array}{r}7.9 \\
(7.4 ; 8.5)\end{array}$ & $\begin{array}{r}6.2 \\
(5.5 ; 6.9)\end{array}$ & $\begin{array}{r}6.9 \\
(6.2 ; 7.6)\end{array}$ & $\begin{array}{r}7.2 \\
(6.9 ; 7.5)\end{array}$ & $\begin{array}{r}7.0 \\
(6.8 ; 7.3)\end{array}$ \\
\hline Inactive(\%) & $\begin{array}{r}53.4 \\
(47.9 ; 58.9)\end{array}$ & $\begin{array}{r}53 \cdot 3 \\
(47.6 ; 59.0)\end{array}$ & $\begin{array}{r}27.7 \\
(22.0 ; 33.3)\end{array}$ & $\begin{array}{r}33.4 \\
(27.5 ; 39.3)\end{array}$ & $\begin{array}{r}65.1 \\
(59.4 ; 70.7)\end{array}$ & $\begin{array}{r}66.1 \\
(60.4 ; 71.9)\end{array}$ & $\begin{array}{r}47.9 \\
(41.6 ; 54.2)\end{array}$ & $\begin{array}{r}43.9 \\
(37.8 ; 50.0)\end{array}$ & $\begin{array}{r}61.8 \\
(54.5 ; 69.1)\end{array}$ & $\begin{array}{r}52.8^{-} \\
(45 \cdot 3 ; 60.2)\end{array}$ & $\begin{array}{r}54.4 \\
(51.6 ; 57.1)\end{array}$ & $\begin{array}{r}54.0 \\
(51.2 ; 56.8)\end{array}$ \\
\hline $\begin{array}{l}\text { Highly inactive }{ }^{\#} \\
(\%)\end{array}$ & $\begin{array}{r}2.0 \\
(0.5 ; 3.6)\end{array}$ & $\begin{array}{r}1.4 \\
(0.1 ; 2.7)\end{array}$ & $\begin{array}{r}1.3 \\
(0.0 ; 2.7)\end{array}$ & $\begin{array}{r}1.7 \\
(0.1 ; 3 \cdot 4)\end{array}$ & $\begin{array}{r}4.2 \\
(1.8 ; 6.6)\end{array}$ & $\begin{array}{r}4.3 \\
(1.8: 6.8)\end{array}$ & $\begin{array}{r}2.7 \\
(0.6 ; 4.7)\end{array}$ & $\begin{array}{r}1.0 \\
(0.0 ; 2.3)\end{array}$ & $\begin{array}{r}3.9 \\
(1.0 ; 6.8)\end{array}$ & $\begin{array}{r}2.1 \\
(0.0 ; 4 \cdot 2)\end{array}$ & $\begin{array}{r}3.1 \\
(2.1 ; 4.0)\end{array}$ & $\begin{array}{r}2.7 \\
(1.8 ; 3.6)\end{array}$ \\
\hline Active(\%) & $\begin{array}{r}46.6 \\
(41.1 ; 52.1)\end{array}$ & $\begin{array}{r}46.7 \\
(41.0 ; 52.4)\end{array}$ & $\begin{array}{r}72.3 \\
(66.7 ; 78.0)\end{array}$ & $\begin{array}{r}66.6 \\
(60.7 ; 72.5)\end{array}$ & $\begin{array}{r}34.9 \\
(29.3 ; 40.6)\end{array}$ & $\begin{array}{r}33.9 \\
(28.1 ; 39.6)\end{array}$ & $\begin{array}{r}52.1 \\
(45.8 ; 58.4)\end{array}$ & $\begin{array}{r}56.1 \\
(50.0 ; 62.2)\end{array}$ & $\begin{array}{r}38.2 \\
(30.9 ; 45.5)\end{array}$ & $\begin{array}{r}47.2^{-} \\
(39.8 ; 54.7)\end{array}$ & $\begin{array}{r}45.6 \\
(42.9 ; 48.4)\end{array}$ & $\begin{array}{r}46.0 \\
(43.2 ; 48.8)\end{array}$ \\
\hline
\end{tabular}

Note: $\quad-p<0.10$ : Differences between survey years 2011 and 2014 using t-tests and Chi-square tests

\#Subgroup of inactive that do not add up to $100 \%$.

@Weighted according to population size in the five Nordic countries.

Table 51: Mean and proportion $(95 \% \mathrm{Cl})$ for estimates of physical activity among girls in the Nordic countries. NORMO 2011 and 2014

\begin{tabular}{|c|c|c|c|c|c|c|c|c|c|c|c|c|}
\hline & \multicolumn{2}{|c|}{ Denmark } & \multicolumn{2}{|c|}{ Finland } & \multicolumn{2}{|c|}{ Sweden } & \multicolumn{2}{|c|}{ Iceland } & \multicolumn{2}{|c|}{ Norway } & \multicolumn{2}{|c|}{ Nordic region@ } \\
\hline & $\begin{array}{r}2011 \\
(n=288)\end{array}$ & $\begin{array}{r}2014 \\
(n=302)\end{array}$ & $\begin{array}{r}2011 \\
(n=253)\end{array}$ & $\begin{array}{r}2014 \\
(n=257)\end{array}$ & $\begin{array}{r}2011 \\
(n=227)\end{array}$ & $\begin{array}{r}2014 \\
(n=241)\end{array}$ & $\begin{array}{r}2011 \\
(n=266)\end{array}$ & $\begin{array}{r}2014 \\
(n=255)\end{array}$ & $\begin{array}{r}2011 \\
(n=184)\end{array}$ & $\begin{array}{r}2014 \\
(n=185)\end{array}$ & $\begin{array}{r}2011 \\
(n=1,218)\end{array}$ & $\begin{array}{r}2014 \\
(n=1,240)\end{array}$ \\
\hline MVPA (h/wk) & $\begin{array}{r}6.7 \\
(6.2 ; 7 \cdot 3)\end{array}$ & $\begin{array}{r}5 \cdot 7^{* *} \\
(5 \cdot 3 ; 6.1)\end{array}$ & $\begin{array}{r}6.9 \\
(6.377 .4)\end{array}$ & $\begin{array}{r}7.6^{-} \\
(7.0 ; 8.1)\end{array}$ & $\begin{array}{r}4.9 \\
(4.555 \cdot 4)\end{array}$ & $\begin{array}{r}5.2 \\
(4.7 ; 5.7)\end{array}$ & $\begin{array}{r}7.1 \\
(6.6 ; 7.6)\end{array}$ & $\begin{array}{r}7.1 \\
(6.5 ; 7.6)\end{array}$ & $\begin{array}{r}5.3 \\
(4.6 ; 5.9)\end{array}$ & $\begin{array}{r}5.4 \\
(4.9 ; 5.9)\end{array}$ & $\begin{array}{r}5.9 \\
(5.6 ; 6.1)\end{array}$ & $\begin{array}{r}5.9 \\
(5.7 ; 6.1)\end{array}$ \\
\hline Inactive(\%) & $\begin{array}{r}58.0 \\
(52.3 ; 63.7)\end{array}$ & $\begin{array}{r}65.2^{-} \\
(59.8 ; 70.5)\end{array}$ & $\begin{array}{r}54.6 \\
(48.5 ; 60.7)\end{array}$ & $\begin{array}{r}49.0 \\
(42.9 ; 55.1)\end{array}$ & $\begin{array}{r}76.9 \\
(71.4 ; 82.4)\end{array}$ & $\begin{array}{r}71.7 \\
(66.0 ; 77 \cdot 5)\end{array}$ & $\begin{array}{r}50.4 \\
(44.4 ; 56.4)\end{array}$ & $\begin{array}{r}51.5 \\
(45 \cdot 3 ; 57.6)\end{array}$ & $\begin{array}{r}73.5 \\
(67.1 ; 79.8)\end{array}$ & $\begin{array}{r}66.4 \\
(59.6 ; 73.2)\end{array}$ & $\begin{array}{r}66.8 \\
(64.1 ; 69.4)\end{array}$ & $\begin{array}{r}64.2 \\
(61.5 ; 66.9)\end{array}$ \\
\hline $\begin{array}{l}\text { Highly inactive } \\
\text { (\%) }\end{array}$ & $\begin{array}{r}1.9 \\
(0.3 ; 3.5)\end{array}$ & $\begin{array}{r}3.1 \\
(1.1 ; 5.0)\end{array}$ & $\begin{array}{r}0.5 \\
(0.0 ; 1.3)\end{array}$ & $\begin{array}{r}1.7 \\
(0.1 ; 3.4)\end{array}$ & $\begin{array}{r}7.1 \\
(3.7 ; 10.4)\end{array}$ & $\begin{array}{r}3.9 \\
(1.5: 6.4)\end{array}$ & $\begin{array}{r}0.5 \\
(0.0 ; 1.4)\end{array}$ & $\begin{array}{r}4 \cdot 4^{* *} \\
(1.9 ; 6.9)\end{array}$ & $\begin{array}{r}3.3 \\
(0.7 ; 5.8)\end{array}$ & $\begin{array}{r}0.5^{*} \\
(0.0 ; 1.5)\end{array}$ & $\begin{array}{r}3.6 \\
(2.5 ; 4.6)\end{array}$ & $\begin{array}{r}2.2 \\
(1.4 ; 3.0)\end{array}$ \\
\hline Active(\%) & $\begin{array}{r}42.0 \\
(36.3 ; 47.7)\end{array}$ & $\begin{array}{r}34.8^{-} \\
(29.5 ; 40.2)\end{array}$ & $\begin{array}{r}45.4 \\
(39.351 .5)\end{array}$ & $\begin{array}{r}51.0 \\
(44.9 ; 57.1)\end{array}$ & $\begin{array}{r}23.1 \\
(17.6 ; 28.6)\end{array}$ & $\begin{array}{r}28.3 \\
(22.5 ; 34.0)\end{array}$ & $\begin{array}{r}49.6 \\
(43.6 ; 55.6)\end{array}$ & $\begin{array}{r}48.5 \\
(42.4 ; 54.7)\end{array}$ & $\begin{array}{r}26.5 \\
(20.2 ; 32.9)\end{array}$ & $\begin{array}{r}33.6 \\
(26.8 ; 40.4)\end{array}$ & $\begin{array}{r}33.2 \\
(30.6 ; 35.9)\end{array}$ & $\begin{array}{r}35.8 \\
(33.1 ; 38.5)\end{array}$ \\
\hline
\end{tabular}

Note:

- $p<0.10,{ }^{*} p<0.05,{ }^{* *} p<0.01$ : Differences between survey years 2011 and 2014 using t-tests and Chi-square tests.

"Subgroup of inactive that do not add up to $100 \%$.

@Weighted according to population size in the five Nordic countries. 


\section{Sedentary behaviour}

The proportion with high recreational screen time was higher in boys than in girls in the Nordic region (18.2\% vs. $12.8 \%$ ). A similar gender difference was observed in each of the five Nordic countries, but the difference was only significant in Finland.

Between 2011 and 2014, mean recreational screen time decreased in girls in the Nordic region while no change was found in boys (Table 52 and 53 ). Still, the pattern of screen time changed in a similar way in boys and girls: TV time decreased and computer time increased. Mean TV time decreased however more than mean computer time increased non-significantly in girls, while changes in TV time and computer time were of the same magnitude in boys. Analyses in each of the five Nordic countries showed an increase in recreational screen time from 2011 to 2014 in boys in Denmark. The reduced screen time in girls was reflected in a decrease from 2011 to 2014 in the proportion with high screen time in the Nordic region. No change was seen in boys. Data in each of the five Nordic countries did only show a decrease in the proportion with high recreational screen time in girls in Norway. Results on sedentary behaviour in boys and girls were therefore not as clear in each of the five Nordic countries as in the combined Nordic region. 
Table 52: Mean and proportion ( $95 \% \mathrm{Cl}$ ) for estimates of sedentary behaviour among boys in the Nordic countries. NORMO 2011 and 2014

\begin{tabular}{|c|c|c|c|c|c|c|c|c|c|c|c|c|}
\hline & \multicolumn{2}{|c|}{ Denmark } & \multicolumn{2}{|c|}{ Finland } & \multicolumn{2}{|c|}{ Sweden } & \multicolumn{2}{|c|}{ Iceland } & \multicolumn{2}{|c|}{ Norway } & \multicolumn{2}{|c|}{ Nordic region@ } \\
\hline & $\begin{array}{r}2011 \\
(n=313)\end{array}$ & $\begin{array}{r}2014 \\
(n=287)\end{array}$ & $\begin{array}{r}2011 \\
(n=239)\end{array}$ & $\begin{array}{r}2014 \\
(n=239)\end{array}$ & $\begin{array}{r}2011 \\
(n=257)\end{array}$ & $\begin{array}{r}2014 \\
(n=253)\end{array}$ & $\begin{array}{r}2011 \\
(n=238)\end{array}$ & $\begin{array}{r}2014 \\
(n=254)\end{array}$ & $\begin{array}{r}2011 \\
(n=159)\end{array}$ & $\begin{array}{r}2014 \\
(n=162)\end{array}$ & $\begin{array}{r}2011 \\
(n=1,206)\end{array}$ & $\begin{array}{r}2014 \\
(n=1,195)\end{array}$ \\
\hline $\begin{array}{l}\text { Recreational } \\
\text { screen time }(h / d)\end{array}$ & $\begin{array}{r}3.0 \\
(2.8 ; 3.3)\end{array}$ & $\begin{array}{r}3.6^{* *} \\
(3.3 ; 4.0)\end{array}$ & $\begin{array}{r}3.2 \\
(2.8 ; 3.6)\end{array}$ & $\begin{array}{r}3.1 \\
(2.8 ; 3.5)\end{array}$ & $\begin{array}{r}3.3 \\
(3.0 ; 3.6)\end{array}$ & $\begin{array}{r}3.0^{-} \\
(2.7 ; 3.2)\end{array}$ & $\begin{array}{r}2.7 \\
(2.4 ; 3.0)\end{array}$ & $\begin{array}{r}2.4 \\
(2.2 ; 2.6)\end{array}$ & $\begin{array}{r}3.5 \\
(3.0 ; 3.9)\end{array}$ & $\begin{array}{r}3.4 \\
(2.9 ; 3.9)\end{array}$ & $\begin{array}{r}3.2 \\
(3.1 ; 3.4)\end{array}$ & $\begin{array}{r}3.2 \\
(3.1 ; 3.4)\end{array}$ \\
\hline $\mathrm{TV}(\mathrm{h} / \mathrm{d})$ & $\begin{array}{r}1.8 \\
(1.6 ; 2.0)\end{array}$ & $\begin{array}{r}2.0 \\
(1.8 ; 2.2)\end{array}$ & $\begin{array}{r}2.2 \\
(1.8 ; 2.5)\end{array}$ & $\begin{array}{r}1.7^{*} \\
(1.5 ; 2.0)\end{array}$ & $\begin{array}{r}2.2 \\
(1.9 ; 2.4)\end{array}$ & $\begin{array}{r}1.6^{* * *} \\
(1.4 ; 1.8)\end{array}$ & $\begin{array}{r}1.5 \\
(1.3 ; 1.7)\end{array}$ & $\begin{array}{r}1.3^{*} \\
(1.1 ; 1.4)\end{array}$ & $\begin{array}{r}2.1 \\
(1.8 ; 2.4)\end{array}$ & $\begin{array}{r}1.6^{* *} \\
(1.3 ; 1.8)\end{array}$ & $\begin{array}{r}2.1 \\
(2.0 ; 2.2)\end{array}$ & $\begin{array}{r}1.7^{* * *} \\
(1.6 ; 1.8)\end{array}$ \\
\hline Computer (h/d) & $\begin{array}{r}1.3 \\
(1.2 ; 1.4)\end{array}$ & $\begin{array}{r}1.7^{* *} \\
(1.5 ; 1.9)\end{array}$ & $\begin{array}{r}1.0 \\
(0.8 ; 1.2)\end{array}$ & $\begin{array}{r}1.4^{* *} \\
(1.2 ; 1.6)\end{array}$ & $\begin{array}{r}1.2 \\
(1.1 ; 1.4)\end{array}$ & $\begin{array}{r}1.4 \\
(1.2 ; 1.5)\end{array}$ & $\begin{array}{r}1.2 \\
(1.0 ; 1.3)\end{array}$ & $\begin{array}{r}1.1 \\
(1.0 ; 1.3)\end{array}$ & $\begin{array}{r}1.4 \\
(1.2 ; 1.6)\end{array}$ & $\begin{array}{r}2.0^{* *} \\
(1.6 ; 2.4)\end{array}$ & $\begin{array}{r}1.2 \\
(1.2 ; 1.3)\end{array}$ & $\begin{array}{r}1.6^{* *} \\
(1.5 ; 1.7)\end{array}$ \\
\hline $\begin{array}{l}\text { High recreational } \\
\text { screen time (>4 } \\
\text { h/d) (\%) }\end{array}$ & $\begin{array}{r}16.3 \\
(12.2 ; 20.4)\end{array}$ & $\begin{array}{r}22.6^{-} \\
(17 \cdot 7 ; 27.4)\end{array}$ & $\begin{array}{r}17.1 \\
(12.3 ; 21.8)\end{array}$ & $\begin{array}{r}18.6 \\
(13.7 ; 23.6)\end{array}$ & $\begin{array}{r}16.3 \\
(11.8 ; 20.9)\end{array}$ & $\begin{array}{r}15.3 \\
(10.9 ; 19.7)\end{array}$ & $\begin{array}{r}10.5 \\
(6.6 ; 14.3)\end{array}$ & $\begin{array}{r}6.5 \\
(3.5 ; 9.5)\end{array}$ & $\begin{array}{r}20.9 \\
(14.6 ; 27.2)\end{array}$ & $\begin{array}{r}19.4 \\
(13.0 ; 25.8)\end{array}$ & $\begin{array}{r}17.3 \\
(15.1 ; 19.4)\end{array}$ & $\begin{array}{r}18.2 \\
(16.0 ; 20.4)\end{array}$ \\
\hline
\end{tabular}

Note: $\quad-p<0.10,{ }^{*} p<0.05,{ }^{* *} p<0.01,{ }^{* * *} p<0.001$ : Differences between survey years 2011 and 2014 using t-tests and Chi-square tests.

@Weighted according to population size in the five Nordic countries.

Table 53: Mean and proportion $(95 \% \mathrm{Cl})$ for estimates of sedentary behaviour among girls in the Nordic countries. NORMO 2011 and 2014

\begin{tabular}{|c|c|c|c|c|c|c|c|c|c|c|c|c|}
\hline & \multicolumn{2}{|c|}{ Denmark } & \multicolumn{2}{|c|}{ Finland } & \multicolumn{2}{|c|}{ Sweden } & \multicolumn{2}{|c|}{ Iceland } & \multicolumn{2}{|c|}{ Norway } & \multicolumn{2}{|c|}{ Nordic region@ } \\
\hline & $\begin{array}{r}2011 \\
(n=286)\end{array}$ & $\begin{array}{r}2014 \\
(n=301)\end{array}$ & $\begin{array}{r}2011 \\
(n=249)\end{array}$ & $\begin{array}{r}2014 \\
(n=251)\end{array}$ & $\begin{array}{r}2011 \\
(n=221)\end{array}$ & $\begin{array}{r}2014 \\
(n=239)\end{array}$ & $\begin{array}{r}2011 \\
(n=266)\end{array}$ & $\begin{array}{r}2014 \\
(n=254)\end{array}$ & $\begin{array}{r}2011 \\
(n=173)\end{array}$ & $\begin{array}{r}2014 \\
(n=179)\end{array}$ & $\begin{array}{r}2011 \\
(n=1,195)\end{array}$ & $\begin{array}{r}2014 \\
(n=1,224)\end{array}$ \\
\hline $\begin{array}{l}\text { Recreational } \\
\text { screen time }(\mathrm{h} / \mathrm{d})\end{array}$ & $\begin{array}{r}3.2 \\
(2.9 ; 3.4)\end{array}$ & $\begin{array}{r}3.2 \\
(2.9 ; 3.5)\end{array}$ & $\begin{array}{r}3.0 \\
(2.6 ; 3.3)\end{array}$ & $\begin{array}{r}2.6 \\
(2.3 ; 2.9)\end{array}$ & $\begin{array}{r}2.9 \\
(2.6 ; 3.2)\end{array}$ & $\begin{array}{r}2.5^{-} \\
\left(2.3 i^{2} .8\right)\end{array}$ & $\begin{array}{r}2.1 \\
(1.9 ; 2.3)\end{array}$ & $\begin{array}{r}1.9 \\
(1.8 ; 2.1)\end{array}$ & $\begin{array}{r}3.4 \\
(3.0 ; 3.9)\end{array}$ & $\begin{array}{r}2.9^{-} \\
(2.5 ; 3 \cdot 3)\end{array}$ & $\begin{array}{r}3.1 \\
(2.9 ; 3.2)\end{array}$ & $\begin{array}{r}2.8^{* *} \\
(2.6 ; 2.9)\end{array}$ \\
\hline $\operatorname{TV}(\mathrm{h} / \mathrm{d})$ & $\begin{array}{r}2.0 \\
(1.8 ; 2.3)\end{array}$ & $\begin{array}{r}1.7^{*} \\
(1.4 ; 1.9)\end{array}$ & $\begin{array}{r}2.0 \\
(1.8 ; 2.3)\end{array}$ & $\begin{array}{r}1.3^{* * *} \\
(1.1 ; 1.5)\end{array}$ & $\begin{array}{r}1.7 \\
(1.5 ; 1.9)\end{array}$ & $\begin{array}{r}1.4^{* *} \\
(1.2 ; 1.5)\end{array}$ & $\begin{array}{r}1.3 \\
(1.1 ; 1.4)\end{array}$ & $\begin{array}{r}1.0^{* *} \\
(1.0 ; 1.1)\end{array}$ & $\begin{array}{r}2.1 \\
(1.8 ; 2.4)\end{array}$ & $\begin{array}{r}1.8 \\
(1.4 ; 2.1)\end{array}$ & $\begin{array}{r}1.9 \\
(1.8 ; 2.0)\end{array}$ & $\begin{array}{r}1.5^{* * \star} \\
(1.4 ; 1.6)\end{array}$ \\
\hline Computer (h/d) & $\begin{array}{r}1.2 \\
(1.0 ; 1.3)\end{array}$ & $\begin{array}{r}1.6^{* * *} \\
(1.4 ; 1.7)\end{array}$ & $\begin{array}{r}0.9 \\
(0.8 ; 1.1)\end{array}$ & $\begin{array}{r}1.3^{* *} \\
(1.1 ; 1.5)\end{array}$ & $\begin{array}{r}1.3 \\
(1.0 ; 1.5)\end{array}$ & $\begin{array}{r}1.2 \\
(1.1 ; 1.3)\end{array}$ & $\begin{array}{r}0.8 \\
(0.7 ; 1.0)\end{array}$ & $\begin{array}{r}0.9 \\
(0.8 ; 1.0)\end{array}$ & $\begin{array}{r}1.4 \\
(1.2 ; 1.6)\end{array}$ & $\begin{array}{r}1.1^{-} \\
(0.9 ; 1.3)\end{array}$ & $\begin{array}{r}1.2 \\
(1.1 ; 1.3)\end{array}$ & $\begin{array}{r}1.3 \\
(1.2 ; 1.3)\end{array}$ \\
\hline $\begin{array}{l}\text { High recreational } \\
\text { screen time (>4 } \\
\text { h/d) (\%) }\end{array}$ & $\begin{array}{r}17.1 \\
(12.7 ; 21.4)\end{array}$ & $\begin{array}{r}17.6 \\
(13.3 ; 21.9)\end{array}$ & $\begin{array}{r}15.5 \\
(11.0 ; 20.0)\end{array}$ & $\begin{array}{r}10.6 \\
(6.8 ; 14.4)\end{array}$ & $\begin{array}{r}16.3 \\
(11.5 ; 21.2)\end{array}$ & $\begin{array}{r}11.2 \\
(7.2 ; 15.3)\end{array}$ & $\begin{array}{r}6.5 \\
(3.5 ; 9.4)\end{array}$ & $\begin{array}{r}4.5 \\
(2.0 ; 7.1)\end{array}$ & $\begin{array}{r}21.5 \\
(15.4 ; 27.6)\end{array}$ & $\begin{array}{r}13.0^{*} \\
(8.0 ; 17.9)\end{array}$ & $\begin{array}{r}17.3 \\
(15.2 ; 19.5)\end{array}$ & $\begin{array}{r}12.8^{* *} \\
(10.9 ; 14.7)\end{array}$ \\
\hline
\end{tabular}

$\quad \quad-p<0.10,{ }^{*} p<0.05,{ }^{* *} p<0.01,{ }^{* * *} p<0.001$ : Differences between survey years 2011 and 2014 using t-tests and Chi-square tests.

@Weighted according to population size in the five Nordic countries. 


\subsubsection{Parental education}

\section{Physical activity}

In 2014, in the Nordic region, the proportion of inactive was higher in children with a medium educated parent than in children with a high educated parent while no difference was found between children with a low educated parent and children with a medium or high educated parent (Table 54). In Iceland, the proportion of inactive was higher in children with a low educated parent than in children with a medium or high educated parent. In the other Nordic countries, the proportion of inactive did not differ in children according to parental education. A small social difference in physical activity (inactive) was found in children in the Nordic region. Social differences are only reported in the Nordic region because it was not possible to assess social inequality in physical activity and sedentary behaviour in all the Nordic countries due to few participants in the low education group.

In the Nordic region, data from 2011 to 2014 showed no change in mean MVPA and in the proportion of inactive and highly inactive in three education groups. The only change in each of the five Nordic countries was a decrease in the proportion of inactive in children with a medium educated parent in Iceland (from $51.7 \%$ to $37.8 \%$ ).

Table 54: Mean and proportion $(95 \% \mathrm{Cl})$ for estimates of physical activity among children according to parental education in the Nordic region. NORMO 2011 and 2014

\begin{tabular}{|c|c|c|c|c|c|c|}
\hline & \multicolumn{6}{|c|}{ Nordic region@ } \\
\hline & \multicolumn{2}{|c|}{ Low } & \multicolumn{2}{|c|}{ Medium } & \multicolumn{2}{|c|}{ High } \\
\hline & $\begin{array}{r}2011 \\
(n=175)\end{array}$ & $\begin{array}{r}2014 \\
(n=144)\end{array}$ & $\begin{array}{r}2011 \\
(n=940)\end{array}$ & $\begin{array}{r}2014 \\
(n=633)\end{array}$ & $\begin{array}{r}2011 \\
(n=1,339)\end{array}$ & $\begin{array}{r}2014 \\
(n=1,673)\end{array}$ \\
\hline MVPA (h/wk) & $\begin{array}{r}7.2 \\
(6.4 ; 8.1)\end{array}$ & $\begin{array}{r}6.6 \\
(5.8 ; 7 \cdot 3)\end{array}$ & $\begin{array}{r}6.3 \\
(6.0 ; 6.6)\end{array}$ & $\begin{array}{r}6.0 \\
(5.6 ; 6.3)\end{array}$ & $\begin{array}{r}6.5 \\
(6.3 ; 6.8)\end{array}$ & $\begin{array}{r}6.6 \\
(6.4 ; 6.8)\end{array}$ \\
\hline Inactive(\%) & $\begin{array}{r}58.4 \\
(48.6 ; 68.3)\end{array}$ & $\begin{array}{r}60.3 \\
(50.2 ; 70.3)\end{array}$ & $\begin{array}{r}62.9 \\
(59.9 ; 65.9)\end{array}$ & $\begin{array}{r}63.9 \\
(60.2 ; 67.6)\end{array}$ & $\begin{array}{r}58.9 \\
(56.3 ; 61.5)\end{array}$ & $\begin{array}{r}57.5 \\
(55.1 ; 59.8)\end{array}$ \\
\hline Highly inactive (\%) & $\begin{array}{r}5.8 \\
(1.1 ; 10.5)\end{array}$ & $\begin{array}{r}2.2 \\
(0.0 ; 5.1)\end{array}$ & $\begin{array}{r}3.4 \\
(2.3 ; 4.5)\end{array}$ & $\begin{array}{r}3.5 \\
(2.0 ; 4.9)\end{array}$ & $\begin{array}{r}1.9 \\
(1.2 ; 2.6)\end{array}$ & $\begin{array}{r}2.0 \\
(1.3 ; 2.7)\end{array}$ \\
\hline Active(\%) & $\begin{array}{r}41.6 \\
(31.7 ; 51.4)\end{array}$ & $\begin{array}{r}39.7 \\
(29.7 ; 49.8)\end{array}$ & $\begin{array}{r}37.1 \\
(34.1 ; 40.1)\end{array}$ & $\begin{array}{r}36.1 \\
(32.4 ; 39.8)\end{array}$ & $\begin{array}{r}41.1 \\
(38.5 ; 43.7)\end{array}$ & $\begin{array}{r}42.5 \\
(40.2 ; 44.9)\end{array}$ \\
\hline $\begin{array}{l}\text { Relative difference } \\
\text { inactive" }(\%)\end{array}$ & - & - & - & - & 1 & -5 \\
\hline
\end{tabular}

Note: @Weighted according to population size in the five Nordic countries

"Relative difference (\%) = [(value High education group - value Low education group)/value High education group]*100. 
The social difference in physical activity did not change from 2011 to 2014 among children in the Nordic region (Figure 6).

Figure 6: The development from 2011 to 2014 in the social difference in physical activity (inactive) among children in the Nordic region. NORMO 2011 and 2014

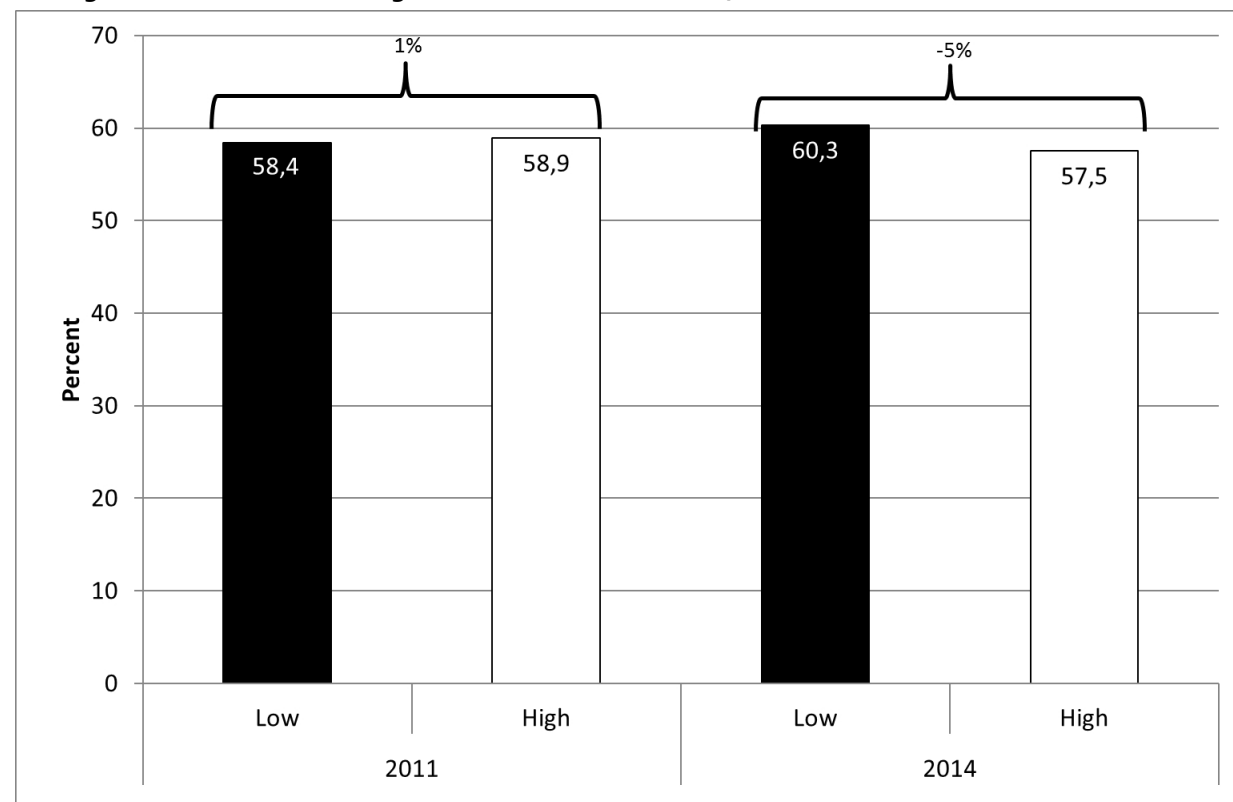

Sedentary behaviour

In 2014, there was no difference in the proportion of children with high recreational screen time across parental education groups in the Nordic region and in Sweden and Norway. In Iceland, the proportion of children with high recreational screen time was higher in children with a low educated parent than in children with a high educated parent. In Denmark, the proportion of children with high recreational screen time was higher in children with a medium educated patent than in children with a high educated parent while the opposite was found in Finland. A small social difference in sedentary behaviour (high recreational screen time) was found among children in the Nordic region. 
Table 55: Table 55: Mean and proportion $(95 \% \mathrm{Cl})$ for estimates of sedentary behaviour among children according to parental education in the Nordic region. NORMO 2011 and 2014

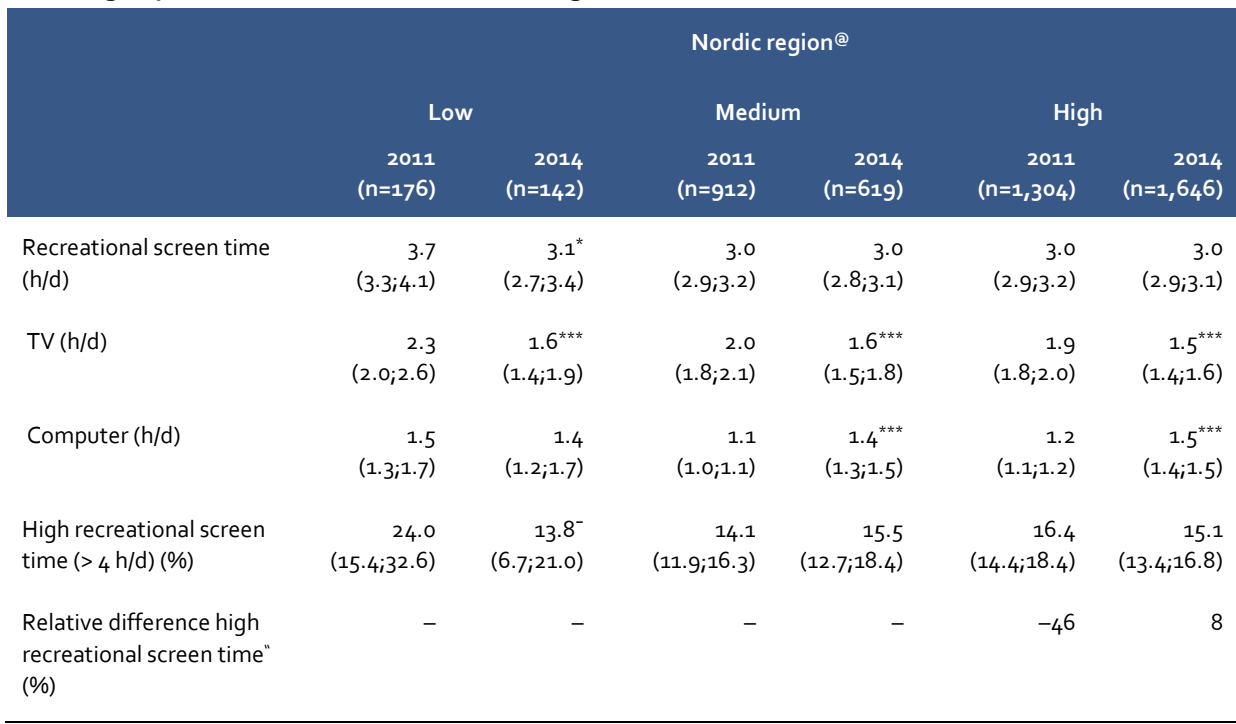

Note: $\quad-p<0.10,{ }^{*} p<0.05,{ }^{* * *} p<0.001$ : Differences between survey years 2011 and 2014 using t-tests and Chi-square tests.

@Weighted according to population size in the five Nordic countries.

"Relative difference (\%) = [(value High education group - value Low education group)/value High education group]* 100 .

Data from 2011 to 2014 showed a decrease in mean TV time in children with a low educated parent in the Nordic region that explained the decrease in mean recreational screen time, but no significant change was detected in the proportion with high recreational screen time (Table 55). Mean recreational screen time and the proportion with high screen time did not change between 2011 and 2014 in children with a medium or high educated parent in the Nordic region. However, the pattern of screen time changed because TV time decreased and computer time increased. The changing screen time pattern was observed in children with a medium or high educated parent in all the Nordic countries, except in Norway. The proportion with high recreational screen time increased in children with a medium educated parent in Denmark (from $17.7 \%$ to $28.4 \%$ ) because of a one hour daily increase in recreational screen time. Moreover, an increase in mean recreational screen time was found in children with a high educated parent in Norway, but no change was found in the proportion with high recreational screen time. 
Between 2011 and 2014, the social difference in sedentary behaviour decreased considerably among children in the Nordic region.

\subsection{Discussion}

\subsubsection{Adults}

The main findings show an increase between 2011 and 2014 in the proportions of highly inactive and with high recreational screen time, albeit the increase in sedentary behaviour was modest in magnitude. This indicates that the lifestyle among adults in the Nordic region has become more inactive and sedentary. The increases in the proportions of highly inactive and/or with high recreational screen time were found in Denmark, Finland and Iceland. The increase in the proportion of highly inactive corresponds to almost 300,000 additional highly inactive $18-65$-y-olds in the Nordic region (from 1.7 milion in 2011 to 2.0 million in 2014). This increase is worrying from a public health point of perspective because of the adverse health effects associated with physical inactivity. The group of highly inactive and sedentary individuals would most likely achieve the greatest health benefits by increased physical activity (NNR 2012).

The proportion of highly active also increased slightly from 2011 to 2014 in the Nordic region. One may therefore speculate if the observation of increased proportions of highly inactive and highly active covers an increased polarisation in the physical activity pattern among adults in the Nordic region. The increased polarisation of physical activity between 2011 and 2014 is most pronounced in men and among the high educated.

The increase between 2011 and 2014 in the proportions of highly inactive or with high recreational screen time was found in women, $18-24$ - and $45-65$-y-olds and in all education groups in the Nordic region. These results suggest that the unfavorable development in physical activity and/or sedentary behaviour apply to the general population. Therefore, further broad public health initiatives may therefore be needed in the Nordic countries that target physical inactivity and sedentary behaviour in the general population. Nevertheless, the low educated may be an especially relevant group to target in the Nordic region because of the high proportions of highly inactive and with high recreational screen time.

Social inequality in physical activity (inactive) did not change among adults in the Nordic region from 2011 to 2014. This looks encouraging at first glance. However, when examining the development in more detail, the proportion of highly inactive increased among the medium and high educated. In addition, social inequality in sedentary behaviour increased because the proportion with high recreational screen time increased 
among the low educated. Overall, results indicate that either physical activity or sedentary behaviour has deteriorated in all social groups in the Nordic region.

The most alarming development of physical activity and sedentary behaviour was found in Iceland. Iceland was the only country where the proportions of highly inactive and with high recreational screen time increased between 2011 and 2014. In contrast, no changes were detected in the proportions of highly inactive and with high recreational screen time in Sweden. These observations may be linked to the OB increase in Iceland and the unchanged OB prevalence in Sweden between 2011 and 2014. Still, OB also increased in Norway and a non-significant upward trend was seen in Denmark and Finland indicating that obesity is a rising public health problem in the Nordic countries.

The development from 2011 to 2014 in physical activity and sedentary behaviour was different in Norway than in the other Nordic countries because Norway was the only country with an upward tendency in physical inactivity (unfavorable development) and a downward tendency in sedentary behaviour (favorable development). Data from Norway should, however, be interpreted with caution due to the low participation rates in both survey years.

\subsubsection{Comparison with other national estimates from the Nordic countries among adults}

Physical activity

Table 56 shows other national population estimates and the recent development of physical activity among adults in the Nordic countries. Other national estimates that cover the time period from 2007 to 2014 are compared with NORMO data to evaluate similarities and differences in the population levels and the development in physical activity and sedentary behaviour among adults. Other national estimates differ from NORMO data with regard to participation rates, sample sizes, survey periods, age groups, assessment methods and defintions of physical activity used. Comparisons between other national estimates and NORMO data should therefore be interpreted with caution. Another important issue related to comparisons between other national estimates and NORMO data is that diet and physical activity are the main topics in NORMO while many of the other national surveys are more general health and disease oriented. Thus, it is possible that participants in the other national surveys have a less healthy lifestyle than participants in NORMO. 
Table 56: Population levels and the development of physical activity among adults in the Nordic countries according to other national estimates

\begin{tabular}{|c|c|c|c|c|c|c|c|c|c|}
\hline & \multicolumn{2}{|c|}{ Denmark } & \multicolumn{2}{|c|}{ Finland } & \multicolumn{2}{|c|}{ Sweden } & \multicolumn{2}{|c|}{ Iceland } & \multirow{3}{*}{$\begin{array}{l}\text { Norway } \\
2008-2009^{x} \\
(20-85 y)\end{array}$} \\
\hline & 2010 & $2011-2013$ & 2011 & 2014 & 2011 & 2014 & 2007 & 2012 & \\
\hline & $(18-75 y)$ & $(18-65 y)$ & \multicolumn{2}{|c|}{$(15-64 y)$} & \multicolumn{2}{|c|}{$(16-84 y)$} & \multicolumn{2}{|c|}{$(18-79 y)$} & \\
\hline Inactive(\%) & 28.6 & 28.9 & 69.7 & 65.8 & 35.0 & 34.0 & 50.0 & N/A & 80.0 \\
\hline Active(\%) & 71.4 & 71.1 & $30.3^{\#}$ & $34.2^{\#}$ & $65.0^{ \pm}$ & $66.0^{\mathrm{E}}$ & $50.0^{ \pm}$ & N/A & 20.0 \\
\hline
\end{tabular}

Note: N/A: Not available.

$\# \geq 4$ times leisure-time phyiscal activity per week; including at least moderate intensity activity for at least 30 minutes each time.

${ }^{\mathrm{E}} \mathrm{MVPA} \geq 30 \mathrm{~min} /$ day.

"Measured data (accelerometri).

Other national estimates displaying the development of physical activity among adults were not available for all the Nordic countries. Only one national survey has been conducted in Norway after year 2000 where objectively measured data on physical activity have been obtained. The population estimates in this survey is therefore not comparable with the self-reported data collected in NORMO. Estimates of physical activity from the most recent survey in Iceland were not available to compare with because data analyses have not been finalised. Finally, questionnaires and definitions of physical activity used in the Nordic surveys differ from what have been used in NORMO. In Denmark, the same questionnaire (NPAQ) was used as in NORMO, which makes the national estimates highly comparable with NORMO data.

The other national estimates show the highest proportion of inactive to be reported in Finland. This is in contrast to NORMO data where Finland is the country with the lowest proportion of inactive. Population levels and rankings of the proportion of inactive in the Nordic countries differ between other national estimates and NORMO data. The proportion of inactive is higher in the Nordic countries according to other national estimates compared with NORMO data, except in Denmark where the proportion of inactive is lower.

Other national estimates from Denmark and Sweden are comparable with NORMO data as the proportion of inactive has not changed in recent years. But other national estimates show a decline in the proportion of inactive in Finland while no change was found with NORMO data.

In summary, the recent development of physical activity in some of the Nordic countries according to other national estimates is in agreement with NORMO data. However, 
the population levels for physical activity in the Nordic countries differ between other national estimates and NORMO data suggesting differences in the representativeness of the samples and the assessment methods and definitions of physical activity used.

\section{Sedentary behaviour}

Only few other comparable national estimates for recreational screen time were available from the Nordic countries. Other national estimates show lower population levels of recreational screen time compared with NORMO data (Table 57). Other national estimates show in accordance with NORMO data an upward tendency in recreational screen time in Finland although the magnitude of the increase was smaller. The development in Denmark was opposite with other national estimates than with NORMO data. Other national estimates show a decrease in recreational screen time in Denmark whereas NORMO data show an increase.

Table 57: Population levels and the development in sedentary behaviour among adults in the Nordic countries according to other national estimates

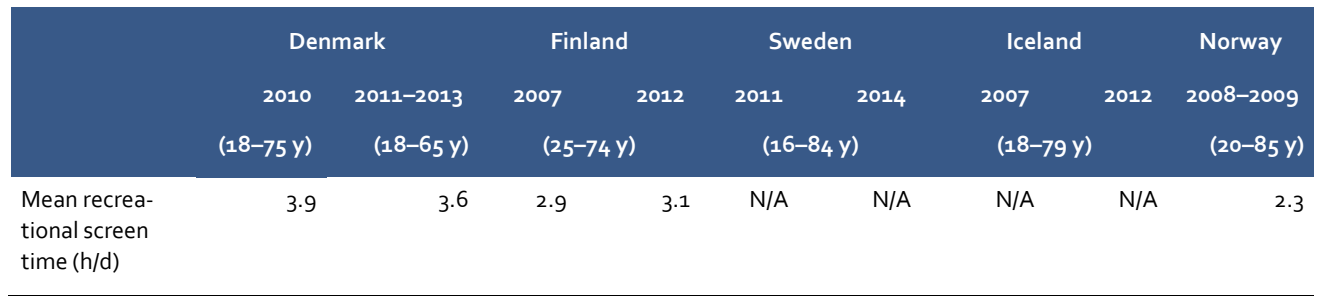

Note: N/A: Not available.

No conclusive remarks were made on sedentary behaviour because only few other comparable national estimates from the Nordic countries were available.

\subsubsection{Evaluation of Goal 2011 and Vision 2021 in the Nordic Plan of Action among adults}

Goal 2011 in the Nordic Plan of Action states that the temporal trend, where an increasing proportion of adults are physically inactive has been brought to a halt and at best reversed (Appendix C). The increase in the proportion of inactive has come to a halt among adults in the Nordic region between 2011 and 2014 because no change was found in the five Nordic countries. This may be regarded as the first step in fulfilling the common Nordic ambition of increased physical activity in the general population. Nevertheless, Goal 2011 may not be considered to be fulfilled among adults because the proportions of highly inactive and/or with high recreational screen time increased in the 
Nordic region and in Denmark, Finland and Iceland. These findings indicate that the lifestyle has become more inactive and sedentary in recent years in the Nordic countries even if the proportion of highly active also has increased.

Vision 2021 states that at least $75 \%$ of adults in the Nordic region should be physically active, i.e they should meet the current physical activity recommendations. In $2014,66 \%$ of the adults in the Nordic region were physically active. Hence, Vision 2021 is currently not fulfilled, but may be possible to fulfill if the level of physical activity increases in the next decade. Finland and Sweden are close to meet the target population level in Vision 2021 while Denmark and Iceland have a longer way to go, and Norway is still far away (Table 37).

\subsubsection{Social inequality}

Goal 2011 may be regarded as fulfilled (Appendic C), because the social difference in physical activity (inactive) has not deepened further among adults in the Nordic region from 2011 to 2014. The development from 2011 to 2014 do, however, not support decreasing social inequality in physical activity in all the Nordic countries because the social difference increased slightly in Sweden. Public health concerns may also be raised on the increasing proportion of highly inactive among the medium and high educated in the Nordic region.

Vision 2021 emphasizes that the social difference in physical activity should decrease and be at most 20\%. Vision 2021 may not be regarded as fulfilled because the social difference in physical activity did not change and is currently $22 \%$. Still, it seems realistic to fulfill Vision 2021 during a seven year period taking the current population level and the development into consideration. At present, Denmark and Finland fulfill Vision 2021 while Sweden, Iceland and Norway do not because of increasing social inequality (Sweden) and/or large social differences in physical activity (Sweden, Iceland, Norway). Iceland and Norway in particular are still far away from fulfilling Vision 2021.

\subsubsection{Children}

There were no changes between 2011 and 2014 in the proportions of inactive and with high recreational screen time among children in the Nordic region. No change or a decrease was also observed in the five Nordic countries. This indicates that level of physical activity and sedentary behaviour has not changed from 2011 to 2014 among children in the Nordic countries. These findings are promising, but the proportion of inactive children in the Nordic region (59\%) is still much too high if the common Nordic ambition about all children being 
physically active in 2021 is going to be fulfilled. Further public health initiatives to increase physical activity in children are therefore needed in the Nordic countries.

In the Nordic region, the proportions of inactive and with high recreational screen time did not change or decreased between 2011 and 2014 in both genders and in all (parental) educational groups. The proportions of highly inactive and with high recreational screen time decreased in girls. These findings are encouraging in the effort to reduce physical inactivity and sedentary time among children.

The social difference in physical activity (inactive) did not change from 2011 to 2014 among children in the Nordic region. The decreasing social difference in sedentary behaviour is promising because this was due to a non-significant decrease among children in the low (parental) education group.

\subsubsection{Comparison with other national estimates from the Nordic countries among children}

\section{Physical activity}

Table 58 shows the population levels and the recent development in physical activity among children in the Nordic countries according to other national estimates. The population levels and the development from 2005 to 2014 according to other national estimates will be compared with NORMO data despite some differences in participation rates, sample sizes, persons who responded the questionnaire, survey periods, age groups and assessment methods used. These differences may be crucial for some of the differences found. Objectively measured physical activity data have been obtained in Norway; the level of physical activity is therefore not comparable with the self-reported NORMO data. In Denmark, the same questionnaire (NPAQ) was used in another national survey as in NORMO. This makes population estimates highly comparable with NORMO data.

Table 58: Population levels and the development in physical activity among children in the Nordic countries according to other national estimates

\begin{tabular}{|c|c|c|c|c|c|c|c|c|c|c|}
\hline & \multicolumn{2}{|c|}{ Denmark } & \multicolumn{2}{|c|}{ Finland } & \multicolumn{2}{|c|}{ Sweden } & \multicolumn{2}{|c|}{ Iceland } & \multicolumn{2}{|c|}{ Norway } \\
\hline & 2010 & $\begin{array}{r}2011- \\
2013\end{array}$ & $\begin{array}{r}2005^{-} \\
2006\end{array}$ & $\begin{array}{r}2009- \\
2010\end{array}$ & $\begin{array}{r}2009- \\
2010\end{array}$ & $\begin{array}{r}2013- \\
2014\end{array}$ & $\begin{array}{r}2009- \\
2010\end{array}$ & $\begin{array}{r}2013^{-} \\
2014\end{array}$ & $\begin{array}{l}2005^{-} \\
2006^{\#}\end{array}$ & $2011^{\#}$ \\
\hline & $(9-17 y)$ & $(7-12 y)$ & \multicolumn{2}{|c|}{ (11 y) } & \multicolumn{2}{|c|}{ (11 y) } & \multicolumn{2}{|c|}{ (11 y) } & \multicolumn{2}{|c|}{$(9 y)$} \\
\hline Inactive(\%) & 56.8 & 61.1 & 57.0 & 68.0 & 82.0 & 82.8 & 79.0 & 73.8 & 16.6 & 22.0 \\
\hline Active(\%) & 43.2 & 38.9 & 43.0 & 32.0 & 18.0 & 17.2 & 21.0 & 26.2 & 83.4 & 78.0 \\
\hline
\end{tabular}

Note: $\quad$ N/A: Not available.

\#Measured data (accelerometri). 
Other national estimates show the highest proportion of inactive in Sweden. This is in line with NORMO data. In contrast to this finding, population levels and ranking of the other Nordic countries differ substantially between other national estimates and NORMO data. Other national estimates show a much higher proportion of inactive in Finland, Sweden and Iceland than in NORMO while similar results were found in Denmark.

Other national estimates and NORMO data show a similar development of physical activity (inactive) in Denmark (upward trend), Sweden and Iceland (no change), but not in Finland and Norway. Other national estimates show an increase in the proportion of inactive in Finland and Norway in contrast to NORMO data that displayed no change or a decrease.

Other national estimates from Finland, Sweden and Iceland are derived from the WHO HBSC study (Currie et al. 2008; Currie et al. 2012; Inchley et al. 2016). A much higher proportion of inactive are observed in HBSC than in NORMO and the development according to the HBSC studies indicate an increase in the proportion of inactive in Finland. These differences could be explained by different survey periods (HBSC: 2005-2010 vs. NORMO: 2011-2014), age groups (HBSC: 11 y vs. NORMO: 7-12 y), persons who responded the questionnaires (HBSC: school child vs. NORMO: parent), and questionnaire used to assess physical activity. The importance of the survey period studied may be highlighted by using Sweden as an example. According to the HBSC studies, the proportion of inactive increased in Sweden from 2005 to 2010 (data not shown) followed by no change between 2010 and 2014. Other national estimates from Finland and Norway cover the time period from 2005 to 2011 and from Denmark, Sweden and Iceland the time period from 2010 to 2014.

In summary, the population levels and the development of physical activity according to other national estimates are not in line with NORMO data. This could be due to differences in survey periods, age groups, persons who responded the questionnaires, and assessment methods.

\section{Sedentary behaviour}

Other comparable national estimates that document the population levels and the most recent development in recreational screen time in the Nordic countries are scarce (Table 59). 
Table 59: Population levels and the development in sedentary behaviour among children in the Nordic countries according to other national estimates

\begin{tabular}{|c|c|c|c|c|c|c|c|c|c|c|}
\hline & \multicolumn{2}{|c|}{ Denmark } & \multicolumn{2}{|c|}{ Finland } & \multicolumn{2}{|c|}{ Sweden } & \multicolumn{2}{|c|}{ Iceland } & \multicolumn{2}{|c|}{ Norway } \\
\hline & 2010 & $\begin{array}{r}2011- \\
2013\end{array}$ & $\begin{array}{r}2005- \\
2006\end{array}$ & $\begin{array}{r}2009- \\
2010\end{array}$ & $\begin{array}{r}2009- \\
2010\end{array}$ & $\begin{array}{r}2013- \\
2014\end{array}$ & $\begin{array}{r}2005- \\
2006\end{array}$ & $\begin{array}{r}2009- \\
2010\end{array}$ & $\begin{array}{r}2005- \\
2006\end{array}$ & 2011 \\
\hline & $(9-17 y)$ & $(7-12 y)$ & \multicolumn{2}{|c|}{ (11y) } & \multicolumn{2}{|c|}{ (11y) } & \multicolumn{2}{|c|}{ (11y) } & \multicolumn{2}{|c|}{$(9 y)$} \\
\hline $\begin{array}{l}\text { Mean recrea- } \\
\text { tional screen } \\
\text { time }(\mathrm{h} / \mathrm{d})\end{array}$ & 3.2 & 2.9 & $N / A$ & $\mathrm{~N} / \mathrm{A}$ & 3.3 & 3.7 & N/A & N/A & N/A & N/A \\
\hline
\end{tabular}

Note: N/A: Not available.

Other comparable national estimates were only available from Denmark and Sweden. Other national estimates were not in line with NORMO data for neither Denmark nor Sweden. The population level of recreational screen time was lower in Denmark and higher in Sweden when comparing other national estimates with NORMO data. Moreover, other national estimates show an opposite development in Denmark and Sweden compared with NORMO data, i.e. mean recreational screen time decreases in Denmark and increases in Sweden according to other national estimates whereas NORMO data showed an increase in Denmark and a decrease in Sweden.

Too few other national estimates from the Nordic countries were available on sedentary behaviour to make meaningful comparison with NORMO data.

\subsubsection{Evaluation of Goal 2011 and Vision 2021 in the Nordic Plan of Action among children}

Goal 2011 and Vision 2021 in the Nordic Plan of Action for physical activity were almost similar for children and adults (Appendix C).

Goal 2011 in the Nordic Plan of Action has been fulfilled among children because the proportions of inactive and with high recreational screen time did not change or even tended to decrease in the Nordic region. The Nordic countries should therefore aim at reducing the proportion of inactive in order to fulfill Vision 2021.

Vision 2021 states that all children should be physically active for at least one hour per day of moderate to vigorous intensity. Vision 2021 does not seem realistic to fulfill taking the current population level and the development in the Nordic countries into consideration. In 2014, it was more common to be physically inactive than active among children in the Nordic region and in particular Sweden, Norway and Denmark are far away from fulfilling Vision 2021 (Table 48). 


\subsubsection{Social inequality}

Goal 2011 states that the social difference in physical activity (inactive) should not have deepened further and at best have been reduced. The social difference in physical activity did not change among children in the Nordic region from 2011 to 2014. Goal 2011 may therefore be regarded as fulfilled.

Vision 2021 states that the social difference in physical activity should decrease and be at most $20 \%$ between different social groups. Vision 2021 may currently be regarded as fulfilled among children in the Nordic region with no change from 2011 to 2014 and a small social difference in physical activity. Still, the high proportion of inactive children in all social groups needs to be reversed. 


\section{Overweight and obesity}

Overweight and obesity are a major public health problem worldwide because of the associated increased risk of non-communicable diseases as cardiovascular diseases, type 2 diabetes and several types of cancers and higher all-cause mortality (WHO 2000, NNR 2012, Global BMI Mortality Collaboration 2016). The prevalence of overweight or obesity (OW/OB) and obesity (OB) have been chosen as key variables for weight status in this chapter where the prevalence and the development from 2011 to 2014 in the Nordic countries are presented. Data on the prevalence and the development from 2011 to 2014 in OW/OB and OB according to age and education in each of the five Nordic countries are commented in this chapter, but data are not tabulated and only significant findings of the development from 2011 to 2014 will be shown in the text.

When comparing OW/OB and $\mathrm{OB}$ between the Nordic countries, only countries where it is possible to identify one or more countries with the highest and/or lowest $\mathrm{OW} / \mathrm{OB}$ and $\mathrm{OB}$ prevalence will be commented in the key findings below.

\subsection{Key findings}

\subsubsection{Adults}

- In 2014, almost one in two adults were overweight or obese and one in eight were obese in the Nordic region. The prevalence of OW/OB and OB was higher in men than in women ( $55.9 \%$ vs. $37.3 \%$, and $14.2 \%$ vs. $11.1 \%)$. The lowest prevalence of $\mathrm{OB}$ was observed in Sweden and the highest prevalence of OW/OB and OB was observed in Iceland.

- In the Nordic region, the prevalence of OW/OB did not change among adults between 2011 and 2014. No change was also found when gender, age and education were analysed. But the prevalence of OB increased from $11.4 \%$ to $12.7 \%$ among adults indicating that the OB prevalence in the Nordic region is not 
levelling off. ${ }^{7}$ The OB prevalence increased in women, $25-44^{-}$and $45-65$-y-olds and among the high educated, but decreased in 18-24-y-olds.

- In the Nordic region, the social differences in OW/OB and OB were large in 2014, but decreased between 2011 and 2014 .

\subsubsection{Children}

- In 2014, more than one in seven children were overweight or obese and $3.2 \%$ were obese in the Nordic region. The prevalence of OW/OB and OB did not differ between genders.

- The prevalence of $O W / O B$ and $O B$ did not change among children between 2011 and 2014 indicating that the $O W / O B$ and $O B$ prevalence in the Nordic region is on a plateau. Also the prevalence of OB did not change from 2011 to 2014 when gender and (parental) education were analysed. However, the prevalence of OW/OB increased from 2011 to 2014 in girls and in children with a high educated parent.

- In the Nordic region, the social difference in OW/OB was large in 2014, but decreased from 2011 to 2014.

\subsubsection{Conclusions}

Data indicate that the $\mathrm{OW} / \mathrm{OB}$ and $\mathrm{OB}$ prevalence among children in the Nordic region has come to a halt, even if the development of OW/OB was not favorable among girls. The OB prevalence among adults in the Nordic region is however not levelling off because OB increased between 2011 and 2014 in all adults and women, but not in men. Goal 2011 in the Nordic Plan of Action has therefore been fulfilled among children, but not among adults. Data also indicate that social differences in $O W / O B$ and $O B$ were large among adults and children, albeit social inequality in OW/OB and OB decreased between 2011 and 2014 .

7 Levelling off implies that an increase was followed by no change. 


\subsection{Results}

\subsubsection{Adults}

In 2014, mean BMI was $25.3 \mathrm{~kg} / \mathrm{m}^{2}$ among adults in the Nordic region which is above the cut-off point for overweight. Results showed that almost one in two adults were overweight or obese and one in eight were obese in 2014 in the Nordic region. Ranking of the prevalence of $O W / O B$ and $O B$ among adults in the five Nordic countries is shown in Table 6o. The prevalence of OW/OB and OB was higher in Iceland than in the other Nordic countries. Moreover, the prevalence of OB was lower in Sweden than in the other Nordic countries.

Table 60: Ranking from lowest to highest prevalence of $O W / O B$ and $O B$ among adults in the five Nordic countries. NORMO 2014

\begin{tabular}{lrlr} 
Country & OW/OB (\%) & Country & OB (\%) \\
Sweden & 44.8 & Sweden & 10.1 \\
Denmark & 47.3 & Norway & 13.3 \\
Norway & 47.5 & Finland & 14.0 \\
Finland & 48.1 & Denmark & 14.8 \\
Iceland & 59.6 & Iceland & 21.0 \\
\hline
\end{tabular}

Table 61 shows the population levels and the development in mean BMI and the $O W / O B$ and $O B$ prevalence among adults in the Nordic countries.

Between 2011 and 2014, an increase in mean BMI was found in the Nordic region and in Iceland and Norway. The prevalence of OB increased from 2011 to 2014 in the Nordic region while no change was observed for OW/OB. When the development in each the five Nordic countries was examined, an increase in OB prevalence was found in Iceland and Norway. 
Table 61: Mean BMI and the prevalence of OW/OB and OB (95\% Cl) among adults in the Nordic countries. NORMO 2011 and 2014

\begin{tabular}{|c|c|c|c|c|c|c|c|c|c|c|c|c|}
\hline & \multicolumn{2}{|c|}{ Denmark } & \multicolumn{2}{|c|}{ Finland } & \multicolumn{2}{|c|}{ Sweden } & \multicolumn{2}{|c|}{ Iceland } & \multicolumn{2}{|c|}{ Norway } & \multicolumn{2}{|c|}{ Nordic region@ } \\
\hline & $\begin{array}{r}2011 \\
(n=2,158)\end{array}$ & $\begin{array}{r}2014 \\
(\mathrm{n}=1,975)\end{array}$ & $\begin{array}{r}2011 \\
(n=1,980)\end{array}$ & $\begin{array}{r}2014 \\
(n=1,696)\end{array}$ & $\begin{array}{r}2011 \\
(n=1,837)\end{array}$ & $\begin{array}{r}2014 \\
(n=1,761)\end{array}$ & $\begin{array}{r}2011 \\
(n=1,942)\end{array}$ & $\begin{array}{r}2014 \\
(n=1,939)\end{array}$ & $\begin{array}{r}2011 \\
(n=1,033)\end{array}$ & $\begin{array}{r}2014 \\
(n=1,015)\end{array}$ & $\begin{array}{r}2011 \\
(n=8,950)\end{array}$ & $\begin{array}{r}2014 \\
(n=8,386)\end{array}$ \\
\hline $\operatorname{BMI}\left(\mathrm{kg} / \mathrm{m}^{2}\right)$ & $\begin{array}{r}25.3 \\
(25.1 ; 25.5)\end{array}$ & $\begin{array}{r}25.5 \\
(25 \cdot 3 ; 25.7)\end{array}$ & $\begin{array}{r}25.5 \\
(25.3 ; 25.7)\end{array}$ & $\begin{array}{r}25.5 \\
(25.3 ; 25.8)\end{array}$ & $\begin{array}{r}25.0 \\
(24.8 ; 25.2)\end{array}$ & $\begin{array}{r}24.9 \\
(24.7 ; 25.1)\end{array}$ & $\begin{array}{r}26.3 \\
(26.1 ; 26.5)\end{array}$ & $\begin{array}{r}26.7^{* *} \\
(26.5 ; 26.9)\end{array}$ & $\begin{array}{r}25.0 \\
(24.7 ; 25.2)\end{array}$ & $\begin{array}{r}25 \cdot 5^{* *} \\
(25.2 ; 25.8)\end{array}$ & $\begin{array}{r}25.2 \\
(25.1 ; 25 \cdot 3)\end{array}$ & $\begin{array}{r}25.3^{*} \\
(25.2 ; 25.4)\end{array}$ \\
\hline OW/OB (\%) & $\begin{array}{r}46.5 \\
(44.4 ; 48.6)\end{array}$ & $\begin{array}{r}47.3 \\
(45.1 ; 49.5)\end{array}$ & $\begin{array}{r}48.1 \\
(45.9 ; 50.3)\end{array}$ & $\begin{array}{r}48.1 \\
(45.7 ; 50.4)\end{array}$ & $\begin{array}{r}45.4 \\
(43.1 ; 47.7)\end{array}$ & $\begin{array}{r}44.8 \\
(42.4 ; 47.1)\end{array}$ & $\begin{array}{r}56.6 \\
(54.4 ; 58.8)\end{array}$ & $\begin{array}{r}59.6^{-} \\
(57.4 ; 61.8)\end{array}$ & $\begin{array}{r}46.6 \\
(43.6 ; 49.6)\end{array}$ & $\begin{array}{r}47.5 \\
(44.4 ; 50.6)\end{array}$ & $\begin{array}{r}46.6 \\
(45.6 ; 47.6)\end{array}$ & $\begin{array}{r}46.7 \\
(45.7 ; 47.8)\end{array}$ \\
\hline $\mathrm{OB}(\%)$ & $\begin{array}{r}12.8 \\
(11.4 ; 14.2)\end{array}$ & $\begin{array}{r}14.8^{-} \\
(13.3 ; 16.4)\end{array}$ & $\begin{array}{r}12.3 \\
(10.8 ; 13.7)\end{array}$ & $\begin{array}{r}14.0 \\
(12.4 ; 15.7)\end{array}$ & $\begin{array}{r}10.9 \\
(9.5 ; 12.3)\end{array}$ & $\begin{array}{r}10.1 \\
(8.7: 11.5)\end{array}$ & $\begin{array}{r}17.7 \\
(16.0 ; 19.4)\end{array}$ & $\begin{array}{r}21.0^{* *} \\
(19.2 ; 22.8)\end{array}$ & $\begin{array}{r}9.1 \\
(7.4 ; 10.9)\end{array}$ & $\begin{array}{r}13.3^{* *} \\
(11.2 ; 15.4)\end{array}$ & $\begin{array}{r}11.4 \\
(10.7 ; 12.0)\end{array}$ & $\begin{array}{r}12.7^{* *} \\
(12.0 ; 13 \cdot 4)\end{array}$ \\
\hline
\end{tabular}

Note: $\quad-p<0.10, * p<0.05, * * p<0.01$ : Differences between survey years 2011 and 2014 using t-tests and Chi-square tests

@Weighted according to population size in the five Nordic countries. 


\subsubsection{Gender}

In 2014, the prevalence of OW/OB was higher in men than in women in the Nordic region ( $55.9 \%$ vs. $37.3 \%$ ) and in the five Nordic countries (Table 62 and 63 ). When differences in $\mathrm{OB}$ prevalence between genders was analysed, a higher prevalence was found in men than in women in the Nordic region (14.2\% vs. $11.1 \%)$ and in Sweden and Finland.

Between 2011 and 2014, mean BMI increased in women in the Nordic region and in women in Iceland and Norway. No change was found in mean BMI in men in the Nordic region and in men in each of the five Nordic countries.

When analysing the development of OW/OB and OB from 2011 to 2014 according to gender, data showed an increase in the prevalence of $\mathrm{OB}$ in women in the Nordic region while no change was found for OW/OB. Data in each of the five Nordic countries showed that the prevalence of $O W / O B$ increased in women in Iceland and the prevalence of $O B$ increased in women in Denmark, Iceland and Norway. No changes were detected in the OW/OB or OB prevalence in men in the Nordic region between 2011 and 2014 and in men in each of the five Nordic countries. 
Table 62: Mean BMI and the prevalence of OW/OB and OB (95\% Cl) among men in the Nordic countries. NORMO 2011 and 2014

\begin{tabular}{|c|c|c|c|c|c|c|c|c|c|c|c|c|}
\hline & \multicolumn{2}{|c|}{ Denmark } & \multicolumn{2}{|c|}{ Finland } & \multicolumn{2}{|c|}{ Sweden } & \multicolumn{2}{|c|}{ Iceland } & \multicolumn{2}{|c|}{ Norway } & \multicolumn{2}{|c|}{ Nordic region@ } \\
\hline & $\begin{array}{r}2011 \\
(n=1,010)\end{array}$ & $\begin{array}{r}2014 \\
(n=973)\end{array}$ & $\begin{array}{r}2011 \\
(n=917)\end{array}$ & $\begin{array}{r}2014 \\
(n=753)\end{array}$ & $\begin{array}{r}2011 \\
(n=874)\end{array}$ & $\begin{array}{r}2014 \\
(n=822)\end{array}$ & $\begin{array}{r}2011 \\
(n=921)\end{array}$ & $\begin{array}{r}2014 \\
(n=887)\end{array}$ & $\begin{array}{r}2011 \\
(n=505)\end{array}$ & $\begin{array}{r}2014 \\
(n=471)\end{array}$ & $\begin{array}{r}2011 \\
(n=4,227)\end{array}$ & $\begin{array}{r}2014 \\
(n=3,906)\end{array}$ \\
\hline BMI (kg/m²) & $\begin{array}{r}26.1 \\
(25.8 ; 26.3)\end{array}$ & $\begin{array}{r}26.1 \\
(25.9 ; 26.4)\end{array}$ & $\begin{array}{r}26.3 \\
(26.1 ; 26.6)\end{array}$ & $\begin{array}{r}26.6 \\
(26.2 ; 26.9)\end{array}$ & $\begin{array}{r}25.9 \\
(25.6 ; 26.2)\end{array}$ & $\begin{array}{r}25.8 \\
(25.5 ; 26.0)\end{array}$ & $\begin{array}{r}27.0 \\
(26.8 ; 27.3)\end{array}$ & $\begin{array}{r}27.1 \\
(26.8 ; 27.4)\end{array}$ & $\begin{array}{r}25.7 \\
(25.4 ; 26.0)\end{array}$ & $\begin{array}{r}26.2^{-} \\
(25.8 ; 26.6)\end{array}$ & $\begin{array}{r}26.0 \\
(25.9 ; 26.1)\end{array}$ & $\begin{array}{r}26.1 \\
(26.0 ; 26.2)\end{array}$ \\
\hline OW/OB (\%) & $\begin{array}{r}56.1 \\
(53.0 ; 59.2)\end{array}$ & $\begin{array}{r}55.9 \\
(52.8 ; 59.1)\end{array}$ & $\begin{array}{r}58.3 \\
(55.1 ; 61.5)\end{array}$ & $\begin{array}{r}60.2 \\
(56.7 ; 63.7)\end{array}$ & $\begin{array}{r}55.3 \\
(52.0 ; 58.6)\end{array}$ & $\begin{array}{r}53.0 \\
(49.6 ; 56.4)\end{array}$ & $\begin{array}{r}66.0 \\
(62.9 ; 69.1)\end{array}$ & $\begin{array}{r}65.8 \\
(62.7 ; 68.9)\end{array}$ & $\begin{array}{r}56.1 \\
(51.8 ; 60.4)\end{array}$ & $\begin{array}{r}56.0 \\
(51.6 ; 60.5)\end{array}$ & $\begin{array}{r}56.4 \\
(54.9 ; 57.9)\end{array}$ & $\begin{array}{r}55.9 \\
(54.3 ; 57.4)\end{array}$ \\
\hline $\mathrm{OB}(\%)$ & $\begin{array}{r}14.3 \\
(12.2 ; 16.5)\end{array}$ & $\begin{array}{r}14.4 \\
(12.2 ; 16.7)\end{array}$ & $\begin{array}{r}14.2 \\
(12.0 ; 16.5)\end{array}$ & $\begin{array}{r}17.4^{-} \\
(14.7 ; 20.2)\end{array}$ & $\begin{array}{r}13.0 \\
(10.7 ; 15.2)\end{array}$ & $\begin{array}{r}12.4 \\
(10.2: 14.7)\end{array}$ & $\begin{array}{r}20.6 \\
(18.0 ; 23.2)\end{array}$ & $\begin{array}{r}21.1 \\
(18.4 ; 23.8)\end{array}$ & $\begin{array}{r}10.1 \\
(7.5 ; 12.7)\end{array}$ & $\begin{array}{r}13.4 \\
(10.4 ; 16.5)\end{array}$ & $\begin{array}{r}13.0 \\
(12.0 ; 14.1)\end{array}$ & $\begin{array}{r}14.2 \\
(13.1 ; 15.3)\end{array}$ \\
\hline
\end{tabular}

Note: $\quad-p<0.10$ : Differences between survey years 2011 and 2014 using t-tests and Chi-square tests.

@Weighted according to population size in the five Nordic countries.

Table 63: Mean BMI and the prevalence of OW/OB and OB (95\% Cl) among women in the Nordic countries. NORMO 2011 and 2014

\begin{tabular}{|c|c|c|c|c|c|c|c|c|c|c|c|c|}
\hline & \multicolumn{2}{|c|}{ Denmark } & \multicolumn{2}{|c|}{ Finland } & \multicolumn{2}{|c|}{ Sweden } & \multicolumn{2}{|c|}{ Iceland } & \multicolumn{2}{|c|}{ Norway } & \multicolumn{2}{|c|}{ Nordic region@ } \\
\hline & $\begin{array}{r}2011 \\
(n=1,148)\end{array}$ & $\begin{array}{r}2014 \\
(n=1,001)\end{array}$ & $\begin{array}{r}2011 \\
(n=1,063)\end{array}$ & $\begin{array}{r}2014 \\
(n=943)\end{array}$ & $\begin{array}{r}2011 \\
(n=963)\end{array}$ & $\begin{array}{r}2014 \\
(n=939)\end{array}$ & $\begin{array}{r}2011 \\
(n=1,021)\end{array}$ & $\begin{array}{r}2014 \\
(n=1,052)\end{array}$ & $\begin{array}{r}2011 \\
(n=528)\end{array}$ & $\begin{array}{r}2014 \\
(n=544)\end{array}$ & $\begin{array}{r}2011 \\
(n=4,723)\end{array}$ & $\begin{array}{r}2014 \\
(n=4,479)\end{array}$ \\
\hline $\operatorname{BMI}\left(\mathrm{kg} / \mathrm{m}^{2}\right)$ & $\begin{array}{r}24.5 \\
(24.2 ; 24.7)\end{array}$ & $\begin{array}{r}24.8 \\
(24.5 ; 25.1)\end{array}$ & $\begin{array}{r}24.7 \\
(24.4 ; 24.9)\end{array}$ & $\begin{array}{r}24.6 \\
(24.3 ; 24.8)\end{array}$ & $\begin{array}{r}24.1 \\
(23.9 ; 24.4)\end{array}$ & $\begin{array}{r}24.0 \\
(23.8 ; 24 \cdot 3)\end{array}$ & $\begin{array}{r}25.5 \\
(25.2 ; 25.8)\end{array}$ & $\begin{array}{r}26.3^{* * *} \\
(26.0 ; 26.6)\end{array}$ & $\begin{array}{r}24.1 \\
(23.8 ; 24.5)\end{array}$ & $\begin{array}{c}24.9^{* *} \\
(24 \cdot 4 ; 25 \cdot 3)\end{array}$ & $\begin{array}{r}24 \cdot 3 \\
(24.2 ; 24 \cdot 4)\end{array}$ & $\begin{array}{r}24 \cdot 5^{*} \\
(24 \cdot 4 ; 24.6)\end{array}$ \\
\hline OW/OB (\%) & $\begin{array}{r}36.5 \\
(33.7 ; 39.3)\end{array}$ & $\begin{array}{r}38.3 \\
(35.3341 .3)\end{array}$ & $\begin{array}{r}38.3 \\
(35.4 ; 41.2)\end{array}$ & $\begin{array}{r}36.6 \\
(33.5 ; 39.7)\end{array}$ & $\begin{array}{r}35.2 \\
(32.2 ; 38.2)\end{array}$ & $\begin{array}{r}36.0 \\
(32.9 ; 39.1)\end{array}$ & $\begin{array}{r}46.8 \\
(43.7 ; 49.8)\end{array}$ & $\begin{array}{r}53.1^{* *} \\
(50.1 ; 56.1)\end{array}$ & $\begin{array}{r}36.5 \\
(32.4 ; 40.6)\end{array}$ & $\begin{array}{r}38.7 \\
(34.6 ; 42.8)\end{array}$ & $\begin{array}{r}36.6 \\
(35.2 ; 37.9)\end{array}$ & $\begin{array}{r}37.3 \\
(35.9 ; 38.8)\end{array}$ \\
\hline $\mathrm{OB}(\%)$ & $\begin{array}{r}11.3 \\
(9.4 ; 13.1)\end{array}$ & $\begin{array}{c}15 \cdot 2^{* *} \\
(13.0 ; 17.4)\end{array}$ & $\begin{array}{r}10.4 \\
(8.6 ; 12.2)\end{array}$ & $\begin{array}{r}10.8 \\
(8.8 ; 12.8)\end{array}$ & $\begin{array}{r}8.8 \\
(7.0 ; 10.6)\end{array}$ & $\begin{array}{r}7.5 \\
(5.8 ; 9.2)\end{array}$ & $\begin{array}{r}14.6 \\
(12.4 ; 16.7)\end{array}$ & $\begin{array}{r}21.0^{* * *} \\
(18.5 ; 23.4)\end{array}$ & $\begin{array}{r}8.1 \\
(5.8 ; 10.4)\end{array}$ & $\begin{array}{r}13.2^{* *} \\
(10.3 ; 16.1)\end{array}$ & $\begin{array}{r}9.6 \\
(8.8 ; 10.5)\end{array}$ & $\begin{array}{r}11.1^{*} \\
(10.2 ; 12.0)\end{array}$ \\
\hline
\end{tabular}

Note: ${ }^{*} \mathrm{p}<0.05,{ }^{* *} \mathrm{p}<0.01,{ }^{* * *} \mathrm{p}<0.001$ : Differences between survey years 2011 and 2014 using t-tests and Chi-square tests.

@Weighted according to population size in the five Nordic countries. 


\subsubsection{Age}

In 2014, the prevalence of OW/OB and OB increased across age groups in the Nordic region (Table 64). The increased prevalence across age groups was found in all the Nordic countries, except in Norway.

Table 64: Mean BMI and the prevalence of OW/OB and $\mathrm{OB}(95 \% \mathrm{Cl})$ among adults according to age in the Nordic region. NORMO 2011 and 2014

\begin{tabular}{|c|c|c|c|c|c|c|}
\hline & \multicolumn{6}{|c|}{ Nordic region@ } \\
\hline & \multicolumn{2}{|c|}{$18-24 \mathrm{Y}$} & \multicolumn{2}{|c|}{$25-44 y$} & \multicolumn{2}{|c|}{$45^{-65 y}$} \\
\hline & 2011 & 2014 & 2011 & 2014 & 2011 & 2014 \\
\hline & $(n=1,018)$ & $(n=825)$ & $(n=3,333)$ & $(n=3,021)$ & $(n=4,599)$ & $(n=4,540)$ \\
\hline BMI $\left(\mathrm{kg} / \mathrm{m}^{2}\right)$ & $\begin{array}{r}23.5 \\
(23.2 ; 23.7)\end{array}$ & $\begin{array}{r}23.2 \\
(23.0 ; 23.4)\end{array}$ & $\begin{array}{r}25.0 \\
(24.8 ; 25.1)\end{array}$ & $\begin{array}{r}25.2^{*} \\
(25 \cdot 1 ; 25 \cdot 4)\end{array}$ & $\begin{array}{r}25.9 \\
(25.8 ; 26.0)\end{array}$ & $\begin{array}{r}26.1^{*} \\
(26.0 ; 26.2)\end{array}$ \\
\hline OW/OB (\%) & $\begin{array}{r}26.8 \\
(24.1 ; 29.5)\end{array}$ & $\begin{array}{r}24.4 \\
(21.5 ; 27.2)\end{array}$ & $\begin{array}{r}43.7 \\
(42.0 ; 45.4)\end{array}$ & $\begin{array}{r}44.5 \\
(42.7 ; 46.3)\end{array}$ & $\begin{array}{r}55.5 \\
(54.1 ; 57.0)\end{array}$ & $\begin{array}{r}56.0 \\
(54.6 ; 57.5)\end{array}$ \\
\hline $\mathrm{OB}(\%)$ & $\begin{array}{r}6.6 \\
(5.1 ; 8.6)\end{array}$ & $\begin{array}{r}3.8^{*} \\
(2.5 ; 5.0)\end{array}$ & $\begin{array}{r}10.5 \\
(9.5 ; 11.5)\end{array}$ & $\begin{array}{r}12.4^{*} \\
(11.2: 13.6)\end{array}$ & $\begin{array}{r}13.7 \\
(12.7 ; 14.7)\end{array}$ & $\begin{array}{r}15.8^{* *} \\
(14.7 ; 16.9)\end{array}$ \\
\hline
\end{tabular}

Note: $\quad{ }^{*} \mathrm{p}<0.05,{ }^{* *} \mathrm{p}<0.01$ : Differences between survey years 2011 and 2014 using $\mathrm{t}$-tests and Chi-square tests. @Weighted according to population size in the five Nordic countries.

Mean BMI increased in 25-44- and 45-65-y-olds from 2011 to 2014 in the Nordic region. Data in each of the five Nordic countries showed that mean BMI decreased in 18-24-yolds in Denmark and increased in 18-24-y-olds in Iceland. Furthermore, mean BMI increased in $25-44-y$-olds in Norway and in 45-65-y-olds in Denmark.

No change was found in the prevalence of OW/OB in the three age groups between 2011 and 2014 in the Nordic region. However, the prevalence of OB decreased almost three percentage points in 18-24-y-olds and increased two percentage points in $25^{-4} 4^{-}$ and 45-65-y-olds (Table 64).

Between 2011 and 2014, data in each of the five Nordic counties revealed an OW/OB decrease in $18-24$-y-olds in Denmark (from $32.4 \%$ to $23.9 \%$ ) and an OB decrease in 18-24-y-olds in Sweden (from 6.3\% to 1.7\%) and Finland (from 8.5\% to 3.4\%). Moreover, OB prevalence increased in $25-44-\mathrm{y}$-olds in Iceland (from $16.3 \%$ to $21.8 \%$ ) and Norway (from 8.9\% to 15.9\%). Finally, OW/OB and OB prevalence increased in 4565 -y-olds in Denmark (OW/OB: from $51.6 \%$ to $56.3 \%$ and OB: from $14.0 \%$ to $17.5 \%$ ). Thus, the prevalence of $O W / O B$ and/or $O B$ appears to decrease in 18-24-y-olds and to increase in 25-44- and 45-65-y-olds in the Nordic countries. 


\subsubsection{Education}

In 2014, an inverse social gradient was found in OW/OB among adults in the Nordic region and in Sweden, i.e. the OW/OB prevalence decrease with increasing level of education (Table 65). In Finland and Norway, the prevalence of OW/OB was higher among the low educated than among the high educated, while in Denmark the prevalence of OW/OB was higher among the low and medium educated than among the high educated. There was no difference in OW/OB prevalence across education groups in Iceland. In addition, the prevalence of $\mathrm{OB}$ was higher among the low and medium educated than among the high educated in the Nordic region and in Denmark, Sweden and Iceland. The prevalence of $\mathrm{OB}$ did not differ between education groups in Finland and Norway. Hence, a lower prevalence of OW/OB and/or OB was found with increasing level of education among adults in the Nordic countries in 2014.

Table 65: Mean $\mathrm{BMI}$ and the prevalence of $\mathrm{OW} / \mathrm{OB}$ and $\mathrm{OB}(95 \% \mathrm{Cl})$ among adults according to education in the Nordic region. NORMO 2011 and 2014

\begin{tabular}{|c|c|c|c|c|c|c|}
\hline & \multicolumn{6}{|c|}{ Nordic region@ } \\
\hline & \multicolumn{2}{|c|}{ Low } & \multicolumn{2}{|c|}{ Medium } & \multicolumn{2}{|c|}{ High } \\
\hline & 2011 & 2014 & 2011 & 2014 & 2011 & 2014 \\
\hline & $(n=1,410)$ & $(n=1,034)$ & $(n=3,898)$ & $(n=3,066)$ & $(n=3,609)$ & $(n=4,270)$ \\
\hline $\operatorname{BMI}\left(\mathrm{kg} / \mathrm{m}^{2}\right)$ & $\begin{array}{r}25.7 \\
(25.5 ; 25.9)\end{array}$ & $\begin{array}{r}25.9 \\
(25.6 ; 26.2)\end{array}$ & $\begin{array}{r}25.5 \\
(25.3 ; 25.6)\end{array}$ & $\begin{array}{r}25.5 \\
(25.3 ; 25.6)\end{array}$ & $\begin{array}{r}24.6 \\
(24.5 ; 24.7)\end{array}$ & $\begin{array}{r}24.7 \\
(24.6 ; 24.8)\end{array}$ \\
\hline OW/OB (\%) & $\begin{array}{r}54.9 \\
(52.0 ; 57.8)\end{array}$ & $\begin{array}{r}53.3 \\
(49.7 ; 56.8)\end{array}$ & $\begin{array}{r}48.9 \\
(47.3 ; 50.5)\end{array}$ & $\begin{array}{r}48.6 \\
(46.8 ; 50.3)\end{array}$ & $\begin{array}{r}39.6 \\
(38.1 ; 41.2)\end{array}$ & $\begin{array}{r}40.6 \\
(39.1 ; 42.0)\end{array}$ \\
\hline OB (\%) & $\begin{array}{r}15.4 \\
(13.3 ; 17.6)\end{array}$ & $\begin{array}{r}15.9 \\
(13.3 ; 18.5)\end{array}$ & $\begin{array}{r}13.1 \\
(12.1 ; 14.2)\end{array}$ & $\begin{array}{r}13.8 \\
(12.6: 15.0)\end{array}$ & $\begin{array}{r}8.0 \\
(7.2 ; 8.9)\end{array}$ & $\begin{array}{r}9.8^{* *} \\
(9.0 ; 10.7)\end{array}$ \\
\hline Relative difference OW/OB\# (\%) & - & - & - & - & -38 & -31 \\
\hline Relative difference OB\# (\%) & - & - & - & - & -93 & -61 \\
\hline
\end{tabular}

Note: $\quad{ }^{* *}$ p <0.01: Differences between survey years 2011 and 2014 using t-tests and Chi-square tests. @Weighted according to population size in the five Nordic countries.

\#Relative difference $(\%)=[($ value High education group - value Low education group $) /$ value High education group]*100.

In 2014, large social differences ( $\geq 20 \%$ ) were found in OW/OB and OB in the Nordic region (Table 65). When analysing each of the five Nordic countries, large social differences in OW/OB were seen in Sweden and Norway, moderate differences (10-20\%) were observed in Denmark and Finland and just a small difference $(\leq 10 \%)$ in Iceland. As regards 
$\mathrm{OB}$, large social differences were observed in Denmark, Sweden and Iceland while moderate and small differences were seen in Finland and Norway, respectively.

Between 2011 and 2014, no change was found in mean BMI according to education in the Nordic region. Analyses in each of the five Nordic countries showed that mean $\mathrm{BMI}$ increased among the low and high educated in Norway and among the medium educated in Iceland.

The prevalence of OB increased two percentage points from 2011 to 2014 among the high educated in the Nordic region while no change occurred among the low and medium educated. The prevalence of OW/OB did not change between 2011 and 2014 in neither of the education groups.

Between 2011 and 2014, data showed an increase in the OW/OB and OB prevalence among the medium educated in Iceland (OW/OB: from $55.6 \%$ to $61.2 \%$ and OB: from $16.0 \%$ to $22.1 \%$ ). Furthermore, a decrease was found in the OB prevalence among the high educated in Sweden (from $7.9 \%$ to $5.5 \%$ ), whereas OB increased among the high educated in Finland (7.4\% to $13.4 \%$ ) and Norway (from $6.8 \%$ to $12.8 \%$ ). Thus, an increase in the $\mathrm{OW} / \mathrm{OB}$ and/or $\mathrm{OB}$ prevalence was found among the medium and/or high educated in Finland, Iceland and Norway whereas a decrease was found in Sweden.

Figure 7: The development from 2011 to 2014 of the social difference in OW/OB among adults in the Nordic region. NORMO 2011 and 2014

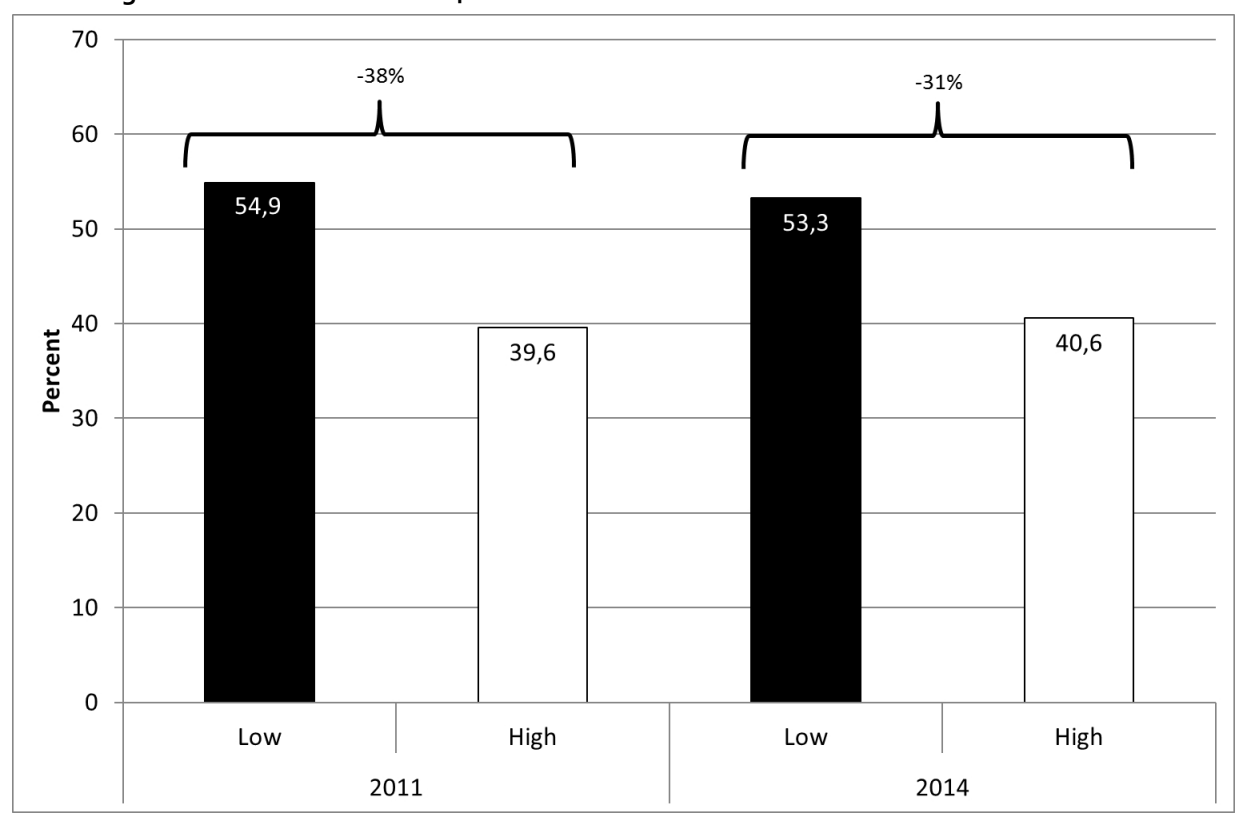


The social difference in OW/OB (Figure 7) and OB decreased from 2011 to 2014 among adults in the Nordic region and in Finland. In the Nordic region, the decreasing social inequality in $\mathrm{OB}$ was mainly caused by an increase in the $\mathrm{OB}$ prevalence in the high education group while in Finland it was caused by a borderline decrease in the low education group and an increase in the high education group. In Denmark, the social difference in $O W / O B$ decreased and increased for $O B$. In Norway an opposite pattern was observed, i.e. the social difference in $O W / O B$ increased whereas the difference in $O B$ decreased slightly. Finally, the social difference in OW/OB did not change in Sweden and Iceland, while the social difference in $\mathrm{OB}$ increased considerably in Sweden and decreased slightly in Iceland. Hence, changes in social inequality in OW/OB and OB were not similar in the five Nordic countries.

\subsubsection{Children}

In 2014, mean BMI was $17.1 \mathrm{~kg} / \mathrm{m}^{2}$ among children in the Nordic region. Results showed that more than one in seven children were overweight or obese and $3.2 \%$ were obese in 2014 in the Nordic region. Ranking of the prevalence of OW/OB and OB among children in the five Nordic countries is shown in Table 66. The prevalence of OW/OB was lower in Denmark than in Norway, Sweden and Iceland, while OB prevalence did not differ between the Nordic countries.

Table 66: Ranking from lowest to highest prevalence of $O W / O B$ and $O B$ among children in the five Nordic countries. NORMO 2014

\begin{tabular}{lcll} 
Country & OW/OB (\%) & Country & OB (\%) \\
Denmark & 11.6 & Finland & 2.3 \\
Finland & 15.6 & Norway & 3.0 \\
Norway & 16.7 & Sweden & 3.2 \\
Sweden & 16.8 & Iceland & 3.2 \\
Iceland & 20.0 & Denmark & 4.2 \\
\hline
\end{tabular}

Table 67 shows the population levels and the development from 2011 to 2014 in mean $\mathrm{BMI}$ and the OW/OB and OB prevalence among children in the Nordic countries. Between 2011 and 2014, mean BMI and the prevalence of OW/OB and OB did not change among children in the Nordic region and in each of the five Nordic countries. 
Table 67: Mean BMI and the prevalence of OW/OB and OB (95\% CI) among children in the Nordic countries. NORMO 2011 and 2014

\begin{tabular}{|c|c|c|c|c|c|c|c|c|c|c|c|c|}
\hline & \multicolumn{2}{|c|}{ Denmark } & \multicolumn{2}{|c|}{ Finland } & \multicolumn{2}{|c|}{ Sweden } & \multicolumn{2}{|c|}{ Iceland } & \multicolumn{2}{|c|}{ Norway } & \multicolumn{2}{|c|}{ Nordic region@ } \\
\hline & $\begin{array}{r}2011 \\
(n=559)\end{array}$ & $\begin{array}{r}2014 \\
(n=531)\end{array}$ & $\begin{array}{r}2011 \\
(n=486)\end{array}$ & $\begin{array}{r}2014 \\
(n=470)\end{array}$ & $\begin{array}{r}2011 \\
(n=453)\end{array}$ & $\begin{array}{r}2014 \\
(n=468)\end{array}$ & $\begin{array}{r}2011 \\
(n=430)\end{array}$ & $\begin{array}{r}2014 \\
(n=399)\end{array}$ & $\begin{array}{r}2011 \\
(n=291)\end{array}$ & $\begin{array}{r}2014 \\
(n=300)\end{array}$ & $\begin{array}{r}2011 \\
(n=2,219)\end{array}$ & $\begin{array}{r}2014 \\
(n=2,168)\end{array}$ \\
\hline $\begin{array}{l}\text { BMI } \\
(\mathrm{kg} / \mathrm{m} 2)\end{array}$ & $\begin{array}{r}17.1 \\
(16.9 ; 17.4)\end{array}$ & $\begin{array}{r}16.9 \\
(16.6 ; 17.2)\end{array}$ & $\begin{array}{r}17.3 \\
(17.1 ; 17.6)\end{array}$ & $\begin{array}{r}17.2 \\
(16.9 ; 17.4)\end{array}$ & $\begin{array}{r}17.3 \\
(17.1 ; 17.6)\end{array}$ & $\begin{array}{r}17.3 \\
(17.0 ; 17.5)\end{array}$ & $\begin{array}{r}17.5 \\
(17.2 ; 17.8)\end{array}$ & $\begin{array}{r}17.4 \\
(17.1 ; 17.6)\end{array}$ & $\begin{array}{r}17.2 \\
(16.9 ; 17.5)\end{array}$ & $\begin{array}{r}17.1 \\
(16.7 ; 17.4)\end{array}$ & $\begin{array}{r}17.3 \\
(17.1 ; 17.4)\end{array}$ & $\begin{array}{r}17.1 \\
(17.0 ; 17.3)\end{array}$ \\
\hline $\begin{array}{l}\text { OW/OB } \\
(\%)\end{array}$ & $\begin{array}{r}12.9 \\
(10.1 ; 15.7)\end{array}$ & $\begin{array}{r}11.6 \\
(8.9 ; 14.3)\end{array}$ & $\begin{array}{r}17.2 \\
(13.9 ; 20.6)\end{array}$ & $\begin{array}{r}15.6 \\
(12.3 ; 18.9)\end{array}$ & $\begin{array}{r}14.8 \\
(11.5 ; 18.1)\end{array}$ & $\begin{array}{r}16.8 \\
(13.4 ; 20.2)\end{array}$ & $\begin{array}{r}20.5 \\
(16.7 ; 24.4)\end{array}$ & $\begin{array}{r}20.0 \\
(16.1 ; 23.9)\end{array}$ & $\begin{array}{r}15.7 \\
(11.6 ; 19.9)\end{array}$ & $\begin{array}{r}16.7 \\
(12.6 ; 20.9)\end{array}$ & $\begin{array}{r}15.1 \\
(13.7 ; 16.6)\end{array}$ & $\begin{array}{r}15.4 \\
(13.9 ; 16.9)\end{array}$ \\
\hline $\mathrm{OB}(\%)$ & $\begin{array}{r}2.4 \\
(1.1 ; 3.7)\end{array}$ & $\begin{array}{r}4.2^{-} \\
(2.5 ; 5.9)\end{array}$ & $\begin{array}{r}3.6 \\
(1.9 ; 5.3)\end{array}$ & $\begin{array}{r}2.3 \\
(1.0 ; 3.7)\end{array}$ & $\begin{array}{r}2.1 \\
(0.8 ; 3.5)\end{array}$ & $\begin{array}{r}3.2 \\
(1.6: 4.8)\end{array}$ & $\begin{array}{r}3.7 \\
(1.9 ; 5.5)\end{array}$ & $\begin{array}{r}3.2 \\
(1.5 ; 5.0)\end{array}$ & $\begin{array}{r}4.7 \\
(2.377 .1)\end{array}$ & $\begin{array}{r}3.0 \\
(1.1 ; 4.9)\end{array}$ & $\begin{array}{r}3.0 \\
(2.3 ; 3.7)\end{array}$ & $\begin{array}{r}3.2 \\
(2.5 ; 3.9)\end{array}$ \\
\hline & $p<c$ & & & & & . & & & & & & \\
\hline
\end{tabular}




\subsubsection{Gender}

In 2014, the prevalence of OW/OB and OB did not differ between genders in the Nordic region and in each of the five Nordic countries.

Between 2011 and 2014, the prevalence of OW/OB increased in girls in the Nordic region while no change was found in boys (Table 68 and 69).

No changes were detected in mean BMI and in the prevalence of OB in boys and girls in the Nordic region. When analysing the development according to gender in each of the five Nordic countries, the prevalence of OB increased in girls in Denmark. Although no significance was established, prevalence data for OW/OB in the five Nordic countries suggest an upward tendency in girls from 2011 to 2014 and a downward tendency in boys. 
Table 68: Mean BMI and the prevalence of OW/OB and OB (95\% Cl) among boys in the Nordic countries. NORMO 2011 and 2014

\begin{tabular}{|c|c|c|c|c|c|c|c|c|c|c|c|c|}
\hline & \multicolumn{2}{|c|}{ Denmark } & \multicolumn{2}{|c|}{ Finland } & \multicolumn{2}{|c|}{ Sweden } & \multicolumn{2}{|c|}{ Iceland } & \multicolumn{2}{|c|}{ Norway } & \multicolumn{2}{|c|}{ Nordic region@ } \\
\hline & $\begin{array}{r}2011 \\
(n=292)\end{array}$ & $\begin{array}{r}2014 \\
(n=265)\end{array}$ & $\begin{array}{r}2011 \\
(n=242)\end{array}$ & $\begin{array}{r}2014 \\
(n=227)\end{array}$ & $\begin{array}{r}2011 \\
(n=249)\end{array}$ & $\begin{array}{r}2014 \\
(n=245)\end{array}$ & $\begin{array}{r}2011 \\
(n=207)\end{array}$ & $\begin{array}{r}2014 \\
(n=198)\end{array}$ & $\begin{array}{r}2011 \\
(n=146)\end{array}$ & $\begin{array}{r}2014 \\
(n=150)\end{array}$ & $\begin{array}{r}2011 \\
(n=1,136)\end{array}$ & $\begin{array}{r}2014 \\
(n=1,085)\end{array}$ \\
\hline $\operatorname{BMI}\left(\mathrm{kg} / \mathrm{m}^{2}\right)$ & $\begin{array}{r}17.3 \\
(16.9 ; 17.6)\end{array}$ & $\begin{array}{r}17.1 \\
(16.7 ; 17.5)\end{array}$ & $\begin{array}{r}17.4 \\
(17.0 ; 17.7)\end{array}$ & $\begin{array}{r}17.2 \\
(16.8 ; 17.5)\end{array}$ & $\begin{array}{r}17.5 \\
(17.1 ; 17.8)\end{array}$ & $\begin{array}{r}17.4 \\
(17.0 ; 17.7)\end{array}$ & $\begin{array}{r}17.5 \\
(17.1 ; 18.0)\end{array}$ & $\begin{array}{r}17.6 \\
(17.2 ; 18.0)\end{array}$ & $\begin{array}{r}17.6 \\
(17.1 ; 18.1)\end{array}$ & $\begin{array}{r}17.4 \\
(17.0 ; 17.9)\end{array}$ & $\begin{array}{r}17.4 \\
(17.3 ; 17.6)\end{array}$ & $\begin{array}{r}17.3 \\
(17.1 ; 17.5)\end{array}$ \\
\hline OW/ OB (\%) & $\begin{array}{r}15.0 \\
(10.9 ; 19.1)\end{array}$ & $\begin{array}{r}11.5 \\
(7.7 ; 15.4)\end{array}$ & $\begin{array}{r}18.1 \\
(13.3 ; 23.0)\end{array}$ & $\begin{array}{r}13.2 \\
(8.8 ; 17.6)\end{array}$ & $\begin{array}{r}17.9 \\
(13.1 ; 22.7)\end{array}$ & $\begin{array}{r}15.8 \\
(11.2 ; 20.3)\end{array}$ & $\begin{array}{r}22.2 \\
(16.5 ; 27.9)\end{array}$ & $\begin{array}{r}20.5 \\
(14.9 ; 26.2)\end{array}$ & $\begin{array}{r}20.2 \\
(13.7 ; 26.6)\end{array}$ & $\begin{array}{r}20.2 \\
(13.8 ; 26.6)\end{array}$ & $\begin{array}{r}17.7 \\
(15.6 ; 19.9)\end{array}$ & $\begin{array}{r}15.3 \\
(13.2 ; 17.3)\end{array}$ \\
\hline $\mathrm{OB}(\%)$ & $\begin{array}{r}3.1 \\
(1.1 ; 5.1)\end{array}$ & $\begin{array}{r}3.2 \\
(1.1 ; 5.3)\end{array}$ & $\begin{array}{r}3.9 \\
(1.4 ; 6.3)\end{array}$ & $\begin{array}{r}1.8 \\
(0.1 ; 3.6)\end{array}$ & $\begin{array}{r}2.2 \\
(0.4 ; 4.0)\end{array}$ & $\begin{array}{r}2.7 \\
(0.7: 4.7)\end{array}$ & $\begin{array}{r}2.4 \\
(0.3 ; 4 \cdot 5)\end{array}$ & $\begin{array}{r}4 \cdot 4 \\
(1.6 ; 7 \cdot 3)\end{array}$ & $\begin{array}{r}6.5 \\
(2.5 ; 10.4)\end{array}$ & $\begin{array}{r}2.7 \\
(0.1 ; 5 \cdot 2)\end{array}$ & $\begin{array}{r}3.6 \\
(2.5 ; 4.6)\end{array}$ & $\begin{array}{r}2.7 \\
(1.7 ; 3.6)\end{array}$ \\
\hline
\end{tabular}

Note:

@Weighted according to population size in the five Nordic countries.

Table 69: Mean BMI and the prevalence of OW/OB and OB (95\% Cl) among girls in the Nordic countries. NORMO 2011 and 2014

\begin{tabular}{|c|c|c|c|c|c|c|c|c|c|c|c|c|}
\hline & \multicolumn{2}{|c|}{ Denmark } & \multicolumn{2}{|c|}{ Finland } & \multicolumn{2}{|c|}{ Sweden } & \multicolumn{2}{|c|}{ Iceland } & \multicolumn{2}{|c|}{ Norway } & \multicolumn{2}{|c|}{ Nordic region@ } \\
\hline & $\begin{array}{r}2011 \\
(n=267)\end{array}$ & $\begin{array}{r}2014 \\
(n=266)\end{array}$ & $\begin{array}{r}2011 \\
(n=244)\end{array}$ & $\begin{array}{r}2014 \\
(n=243)\end{array}$ & $\begin{array}{r}2011 \\
(n=204)\end{array}$ & $\begin{array}{r}2014 \\
(n=223)\end{array}$ & $\begin{array}{r}2011 \\
(n=223)\end{array}$ & $\begin{array}{r}2014 \\
(n=201)\end{array}$ & $\begin{array}{r}2011 \\
(n=145)\end{array}$ & $\begin{array}{r}2014 \\
(n=150)\end{array}$ & $\begin{array}{r}2011 \\
(n=1,083)\end{array}$ & $\begin{array}{r}2014 \\
(n=1,083)\end{array}$ \\
\hline $\mathrm{BMI}\left(\mathrm{kg} / \mathrm{m}^{2}\right)$ & $\begin{array}{r}17.0 \\
(16.7 ; 17.4)\end{array}$ & $\begin{array}{r}16.7 \\
(16.3 ; 17.0)\end{array}$ & $\begin{array}{r}17.3 \\
(16.9 ; 17.6)\end{array}$ & $\begin{array}{r}17.2 \\
(16.8 ; 17.5)\end{array}$ & $\begin{array}{r}17.2 \\
(16.8 ; 17.5)\end{array}$ & $\begin{array}{r}17.2 \\
(16.8 ; 17.6)\end{array}$ & $\begin{array}{r}17.5 \\
(17.1 ; 18.0)\end{array}$ & $\begin{array}{r}17.1 \\
(16.8 ; 17.5)\end{array}$ & $\begin{array}{r}16.8 \\
(16.4 ; 17.2)\end{array}$ & $\begin{array}{r}16.7 \\
(16.2 ; 17.2)\end{array}$ & $\begin{array}{r}17.1 \\
(16.9 ; 17.2)\end{array}$ & $\begin{array}{r}17.0 \\
(16.8 ; 17.2)\end{array}$ \\
\hline OW/OB (\%) & $\begin{array}{r}10.8 \\
(7.0 ; 14.5)\end{array}$ & $\begin{array}{r}11.6 \\
(7.8 ; 15.4)\end{array}$ & $\begin{array}{r}16.2 \\
(11.6 ; 20.8)\end{array}$ & $\begin{array}{r}17.9 \\
(13.1 ; 22.7)\end{array}$ & $\begin{array}{r}11.5 \\
(7.0 ; 16.0)\end{array}$ & $\begin{array}{r}17.9^{-} \\
(12.8 ; 22.9)\end{array}$ & $\begin{array}{r}18.8 \\
(13.7 ; 23.9)\end{array}$ & $\begin{array}{r}19.4 \\
(14.0 ; 24.9)\end{array}$ & $\begin{array}{r}10.9 \\
(5.9 ; 15.9)\end{array}$ & $\begin{array}{r}13.4 \\
(8.1 ; 18.7)\end{array}$ & $\begin{array}{r}12.4 \\
(10.4 ; 14.4)\end{array}$ & $\begin{array}{r}15.6^{*} \\
(13.5 ; 17.7)\end{array}$ \\
\hline OB (\%) & $\begin{array}{r}1.6 \\
(0.1 ; 3.2)\end{array}$ & $\begin{array}{r}5.3^{*} \\
(2.6 ; 7.9)\end{array}$ & $\begin{array}{r}3.3 \\
(1.1 ; 5.5)\end{array}$ & $\begin{array}{r}2.8 \\
(0.7 ; 4.8)\end{array}$ & $\begin{array}{r}2.1 \\
(0.1 ; 4.0)\end{array}$ & $\begin{array}{r}3.7 \\
(1.2: 6.2)\end{array}$ & $\begin{array}{r}5.1 \\
(2.2 ; 8.0)\end{array}$ & $\begin{array}{r}2.1^{-} \\
(0.1 ; 4.1)\end{array}$ & $\begin{array}{r}2.7 \\
(0.1 ; 5.3)\end{array}$ & $\begin{array}{r}3.3 \\
(0.5 ; 6.1)\end{array}$ & $\begin{array}{r}2.4 \\
(1.5 ; 3.3)\end{array}$ & $\begin{array}{r}3.8^{-} \\
(2.7 ; 4.9)\end{array}$ \\
\hline
\end{tabular}

Note: $\quad-p<0.10,{ }^{*} p<0.05$ : Differences between survey years 2011 and 2014 using t-tests and Chi-square tests.

@Weighted according to population size in the five Nordic countries. 


\subsubsection{Parental education}

In 2014, the prevalence of OW/OB was higher in children with a medium educated parent than in children with a high educated parent in the Nordic region (Table 70). Moreover, the prevalence of OB was higher in children with a low educated parent than in children with a high educated parent. There was no difference in the prevalence of $O W / O B$ and $O B$ across parental education groups in each of five Nordic countries, except in Denmark where a higher prevalence of $O B$ was seen in children with a low or medium educated parent than in children with a high educated parent.

In 2014, a moderate social difference in OW/OB and a large difference in OB were found among children in the Nordic region. Social differences are only reported in the Nordic region because it was not possible to assess social inequality in $O W / O B$ and $O B$ in all the Nordic countries due to few participants in the group of children with a low educated parent.

When analysing the development from 2011 to 2014 in mean BMI and in the prevalence of $\mathrm{OW} / \mathrm{OB}$ and $\mathrm{OB}$ according to parental education in the Nordic region and in each of the five Nordic countries, the only significant finding was an increase in the OW/OB prevalence in children with a high educated parent in the Nordic region (Table 70).

Table 70: Mean $\mathrm{BMI}$ and the prevalence of $\mathrm{OW} / \mathrm{OB}$ and $\mathrm{OB}(95 \% \mathrm{Cl})$ among children according to parental education in the Nordic region. NORMO 2011 and 2014

\begin{tabular}{|c|c|c|c|c|c|c|}
\hline & \multicolumn{4}{|c|}{ Nordic region@ } & \multirow{2}{*}{\multicolumn{2}{|c|}{ High }} \\
\hline & \multicolumn{2}{|c|}{ Low } & \multicolumn{2}{|c|}{ Medium } & & \\
\hline & $\begin{array}{r}2011 \\
(n=152)\end{array}$ & $\begin{array}{r}2014 \\
(n=121)\end{array}$ & $\begin{array}{r}2011 \\
(n=844)\end{array}$ & $\begin{array}{r}2014 \\
(\mathrm{n}=553)\end{array}$ & $\begin{array}{r}2011 \\
(n=1,215)\end{array}$ & $\begin{array}{r}2014 \\
(n=1,485)\end{array}$ \\
\hline $\operatorname{BMI}\left(\mathrm{kg} / \mathrm{m}^{2}\right)$ & $\begin{array}{r}18.0 \\
(17.5 ; 18.6)\end{array}$ & $\begin{array}{r}18.0 \\
(17.2 ; 18.8)\end{array}$ & $\begin{array}{r}17.4 \\
(17.3 ; 17.6)\end{array}$ & $\begin{array}{r}17.2^{-} \\
(16.9 ; 17.4)\end{array}$ & $\begin{array}{r}16.9 \\
(16.8 ; 17.1)\end{array}$ & $\begin{array}{r}16.9 \\
(16.7 ; 17.0)\end{array}$ \\
\hline OW/OB (\%) & $\begin{array}{r}22.1 \\
(13.3 ; 30.9)\end{array}$ & $\begin{array}{r}16.4 \\
(8.5 ; 24.2)\end{array}$ & $\begin{array}{r}18.1 \\
(15.5 ; 20.6)\end{array}$ & $\begin{array}{r}17.3 \\
(14.2 ; 20.4)\end{array}$ & $\begin{array}{r}11.2 \\
(9.4 ; 12.9)\end{array}$ & $\begin{array}{r}13.8^{*} \\
(12.0 ; 15.5)\end{array}$ \\
\hline $\mathrm{OB}(\%)$ & $\begin{array}{r}7.6 \\
(2.0 ; 13.2)\end{array}$ & $\begin{array}{r}6.0 \\
(0.9 ; 11.0)\end{array}$ & $\begin{array}{r}3.4 \\
(2.2 ; 4 \cdot 5)\end{array}$ & $\begin{array}{r}3.1 \\
(1.7: 4 \cdot 4)\end{array}$ & $\begin{array}{r}1.7 \\
(1.0 ; 2.4)\end{array}$ & $\begin{array}{r}2.4 \\
(1.7 ; 3.2)\end{array}$ \\
\hline $\begin{array}{l}\text { Relative difference } \\
\text { OW/OB (\%)" }\end{array}$ & - & - & - & - & -98 & -19 \\
\hline Relative difference $\mathrm{OB}(\%)^{\#}$ & - & - & - & - & -345 & -148 \\
\hline
\end{tabular}

Note: $\quad{ }^{*} p<0.05,{ }^{-} p<0.10$ : Differences between survey years 2011 and 2014 using t-tests and Chi-square tests. @Weighted according to population size in the five Nordic countries.

${ }^{\#}$ Relative difference $(\%)=[($ value High education group - value Low education group)/value High education group]* 100 . 
Between 2011 and 2014, the social difference in OW/OB (Figure 8) and OB decreased considerably among children in the Nordic region. The decreasing social difference in OW/OB was caused by a non-significant decrease in the OW/OB prevalence in children with a low educated parent and an increase in children with a high educated parent.

Figure 8: The development from 2011 to 2014 of the social difference in $O W / O B$ among children in the Nordic region. NORMO 2011 and 2014

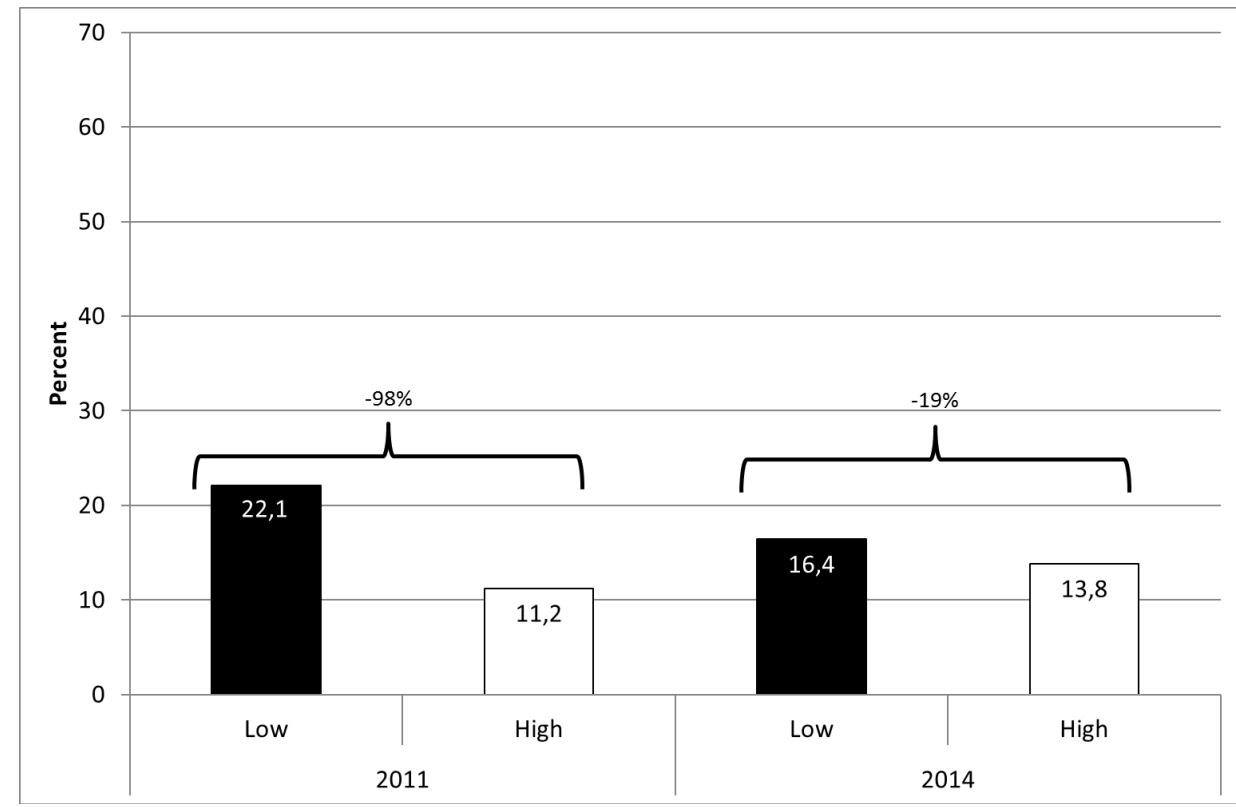

\subsection{Discussion}

\subsubsection{Adults}

Results show an increase in the prevalence of OB - from $11.4 \%$ in 2011 to $12.7 \%$ in 2014 - among adults in the Nordic region. The OB prevalence increased in Iceland and Norway and a non-significant upward tendency was found in Denmark and Finland. Hence, data indicate that the prevalence of $O B$ among adults in the Nordic countries is not levelling off. The $O B$ increase is worrying from a public health perspective because it corresponds to more than 200,000 additional obese adults in the Nordic region during a three year time period (from 1.8 million obese in 2011 to 2.0 million obese in 2014). The $O B$ increase was significant among women, but not among men. This finding is in agreement with recently published data from Denmark where the development of 
OW/OB and OB was reported in adults between 2005 and 2013 (Matthiessen and Stockmarr 2015).

The prevalence of $O B$ increased in 25-44- and 45-65-y-olds in the Nordic region whereas a decrease was observed in $18-24-y$-olds. Social inequality in $O B$ also decreased. This decrease looks encouraging at first glance. However, when examining the figures more closely, it was caused by an $\mathrm{OB}$ increase in the high education group.

The development in the Nordic countries seems to be heading in a direction where it will be more common to be overweight or obese as an adult than being normal weight. The prevalence of OW/OB and OB is most likely underestimated in NORMO because self-reported data has been obtained (Connor Gorber et al. 2007), and the proportion of obese is generally higher among non-participants than participants in population surveys (Nyholm et al. 2008).

The most positive development was seen in Sweden because the prevalence of OB did not change and the prevalence was relative low. In contrast to Sweden, the most alarming development was observed in Iceland because of the large OB increase and the high prevalence leading to more than one in five adults being obese in Iceland. The difference observed in $\mathrm{OB}$ between Sweden and Iceland was most striking among women where the OB prevalence was close to three times higher in women in Iceland compared with Sweden. Iceland is the only Nordic country where it is more common to be overweight or obese as an adult than being normal weight so far.

\subsubsection{Comparison with other national estimates from the Nordic countries among adults}

Table 71 shows the prevalence and the recent development in OW/OB and OB among adults in the Nordic countries according to other national estimates. Despite some differences in participation rates, survey periods, age groups and how height and weight were measured, these national estimates are relevant to compare with NORMO data. Other national estimates cover the period from around year 2006 where the Nordic Plan of Action was launched up to 2014. This time period was selected because Vision 2021 in the Nordic Plan of Action was defined by use of the prevalence of OW/OB and $\mathrm{OB}$ at year 2006. All other national estimates are self-reported, except in Finland where measured estimates have been obtained. 
Table 71: Prevalence and the development of $O W / O B$ and $O B$ among adults in the Nordic countries according to other national estimates

\begin{tabular}{|c|c|c|c|c|c|c|c|c|c|c|}
\hline & \multicolumn{2}{|c|}{ Denmark } & \multicolumn{2}{|c|}{ Finland $^{*}$} & \multicolumn{2}{|c|}{ Sweden } & \multicolumn{2}{|c|}{ Iceland } & \multicolumn{2}{|c|}{ Norway } \\
\hline & $\begin{array}{r}2005 \\
(16\end{array}$ & 2013 & $\begin{array}{r}2006 \\
\left(15^{-}\right.\end{array}$ & $\begin{array}{l}2014 \\
\text { ) }\end{array}$ & $\begin{array}{r}2006 \\
(16-\end{array}$ & $\begin{array}{l}2014 \\
\text { f) }\end{array}$ & $\begin{array}{r}2007 \\
(18-\end{array}$ & 2012 & $\begin{array}{r}2005 \\
(18-\end{array}$ & $\begin{array}{l}2012 \\
\text { y) }\end{array}$ \\
\hline OW/OB (\%) & 44.0 & 47.4 & 47.7 & 50.2 & 46.0 & 49.0 & 60.1 & 63.3 & 45.0 & 46.0 \\
\hline $\mathrm{OB}(\%)$ & 11.4 & 14.1 & 14.3 & 16.3 & 12.0 & 14.0 & 20.1 & 22.2 & 9.0 & 10.0 \\
\hline
\end{tabular}

Note: $\quad{ }^{*}$ Measured data.

The most recent prevalence of $\mathrm{OW} / \mathrm{OB}$ and $\mathrm{OB}$ were higher for all the Nordic countries, except for Denmark, according to other national estimates compared with NORMO data (Table 61). Part of this difference may be explained by higher participation rates and less skewed population distributions (gender, age, education) in the other national surveys. Other recent national estimates found in accordance with NORMO data the highest prevalence of $O W / O B$ and $O B$ among adults in Iceland, but the lowest prevalence in Norway in contrast to Sweden when NORMO data were used.

Between 2005 and 2014, other national estimates show that the Nordic countries have experienced a two to three percentage point increase in OW/OB and OB prevalence, apart from Norway where the observed increase was one percentage point. When comparing the development of OW/OB and $O B$ in each of the five Nordic countries using other national estimates and NORMO data, changes in Denmark and Iceland are similar, but only partly comparable in Finland and Norway and not comparable in Sweden. In Finland, other national estimates show an increase in the prevalence of OW/OB while no change was observed in NORMO. Moreover, a smaller OB increase was found in Norway with other national estimates compared with NORMO data. NORMO data from Norway should be interpreted with caution due to low participation rates in both survey years. Finally, the prevalence of OW/OB and OB increased in Sweden according to other national estimates while NORMO data showed no change between survey years. Still, other recently obtained national estimates support the finding in NORMO of no change in the OW/OB prevalence in Sweden between 2011 and 2014 (data not shown). This highlights that some of the differences may be explained by the different survey periods examined. The comparisons made above underlines the importance of measuring the prevalence and the development in the same period and age groups and using the same assessment method as has been done in NORMO. 
In summary, other national estimates from the Nordic countries display higher prevalence of OW/OB and OB than NORMO data, but find in agreement with NORMO data an overall $O B$ increase over time. Thus, other national estimates from the Nordic countries support NORMO data in that the OB prevalence among adults in the Nordic countries is not levelling off.

\subsubsection{Evaluation of Goal 2011 and Vision 2021 in the Nordic Plan of Action among adults}

Goal 2011 in the Nordic Plan of Action was to stop the increase in the OW/OB and OB prevalence in the Nordic countries (Appendix C). Even if no change in the OW/OB prevalence was detected between 2011 and 2014, Goal 2011 has not been fulfilled because $O B$ continues to rise in the Nordic region. This increase may especially be attributed to women where OB prevalence increased between 2011 and 2014. The development in the five Nordic countries was however not similar because the OB prevalence increased in Iceland and Norway while no change was found in Denmark, Finland and Sweden.

Vision 2021 in the Nordic Plan of Action aims to reduce OW/OB and OB with at least $30 \%$ from the prevalence at year 2006 (Table 72).

Table 72: Target prevalence of OW/OB and OB among adults according to Vision 2021 in the Nordic Plan of Action. Target prevalence corresponds to a $30 \%$ reduction from the prevalence at year 2006

\begin{tabular}{lrrrrrr} 
& Denmark & Finland & Sweden & Iceland & Norway \\
\hline OW/OB (\%) & 30.8 & 33.4 & 32.2 & 42.1 & 31.5 \\
OB (\%) & 8.0 & 10.0 & 8.4 & 14.1 & 6.3 \\
\hline
\end{tabular}

Even though this vision may not be evaluated until 2021, one has to be highly optimistic to believe that it will be fulfilled taking the current prevalence and the development into consideration. In 2014, the prevalence of OW/OB and OB was $45-60 \%$ and $10-21 \%$, respectively, in the Nordic countries and the target prevalence in Vision 2021 is $31-42 \%$ for OW/OB and $6-14 \%$ for OB. Thus, the current prevalence of OW/OB and OB should be reduced significantly in a seven year period to reach the target prevalence in Vision 2021. None of the Nordic countries are close to meet the target prevalence of OW/OB and OB in Vision 2021, except Sweden with regard to OB. 


\subsubsection{Social inequality}

Goal 2011 states that the existing social differences in OW/OB and OB should not have deepened further and at best have been reduced (Appendix $C$ ). The social difference in $O W / O B$ and $O B$ decreased from 2011 to 2014 among adults in the Nordic region. Goal 2011 looks therefore to be fulfilled as social inequality in $O W / O B$ and $O B$ was reduced. Mixed results were found for the development of social inequality in $O W / O B$ and $O B$ in the five Nordic countries. Social inequality in both $O W / O B$ and $O B$ decreased only in Finland between 2011 and 2014

The way Goal 2011 was fulfilled among adults in the Nordic region is not favorable from a public health point of view because it was reduced through an increase in the $O B$ prevalence in the high education group. This means that health status has deteriorated in the high social group. It is therefore important to be aware of the changes in all social groups when the aim is to lower social inequality in health.

Vision 2021 states that social inequality in OW/OB should decrease and that the social difference in OW/OB should be at most $20 \%$. The social difference decreased from $38 \%$ in 2011 to $31 \%$ in 2014 in the Nordic region. Thus in 2014, the social difference in OW/OB was still large among adults in the Nordic region. A large social difference in OW/OB was also seen in Sweden and Norway.

Vision 2021 is not fulfilled yet, but data suggest that the vision may be possible to fulfill. Vision 2021 should also be evaluated with the ambition of achieving better health status in all social groups and not just decrease social inequality in OW/OB. This was however not the case in the Nordic region where a non-significant OW/OB increase was found among the high educated. In Denmark and Finland, an encouraging non-significant decrease in the OW/OB prevalence was observed in the low education group. The OB prevalence also decreased non-significantly in the low education group in Finland, but not in Denmark. In Sweden and Iceland, social inequality in OW/OB did not change from 2011 to 2014, while in Norway, social inequality increased mainly due to a nonsignificant increase in the low education group.

\subsubsection{Children}

Results show that the prevalence of OW/OB and OB has come to a halt between 2011 and 2014 among children in the Nordic region. This was observed in all the Nordic countries and supports the findings from other studies (Rokholm et al. 2010, Olds et al. 2011, Morgen et al. 2013) which show that $O W / O B$ and $O B$ prevalence among children in the Nordic countries is still on a plateau. Even though these findings are encouraging, a relatively high prevalence of OW/OB was observed and a halt has previously been followed by a 
rise. It is therefore important to continue to obtain anthropometric population data and to draw attention to $O B$ prevention among children in the Nordic countries.

The development from 2011 to 2014 differed between boys and girls in the Nordic region because the $O W / O B$ prevalence increased in girls while a non-significant downward tendency was observed in boys. The different development between genders was seen in all the Nordic countries although no significance was established. These findings are in line with a recently published study analysing the development from 2005 to 2013 in OW/OB and OB among children and adolescents in Denmark (Matthiessen and Stockmarr 2015).

Social inequality in $O W / O B$ and $O B$ decreased considerably among children in the Nordic region. The reduced social inequality in OW/OB was caused by a non-significant $\mathrm{OW} / \mathrm{OB}$ decrease in children with a low educated parent and an increase in children with a high educated parent.

\subsubsection{Comparison with other national estimates from the Nordic countries among children}

Other national estimates of the prevalence and the recent development of OW/OB and $\mathrm{OB}$ among children in the Nordic countries are shown in Table 73. Other national estimates differ from NORMO data in terms of participation rates, representativeness, persons who responded the questionnaire, survey periods, age groups and how height and weight were assessed, but are still useful for comparison. Other national estimates cover the time period from 2005 to 2013 in 4-17-y-olds. Estimates from Sweden and Iceland are not nationally representative and measured anthropometric estimates have been obtained in Sweden, Iceland and Norway.

Table 73: Prevalence and the development of OW/OB and OB among children in the Nordic countries according to other national estimates

\begin{tabular}{|c|c|c|c|c|c|c|c|c|c|c|}
\hline & \multicolumn{2}{|c|}{ Denmark } & \multicolumn{2}{|c|}{ Finland } & \multicolumn{2}{|c|}{ Sweden ${ }^{*}$} & \multicolumn{2}{|c|}{ Iceland $d^{\star * \#}$} & \multicolumn{2}{|c|}{ Norway* } \\
\hline & $2005^{-2008}$ & $2011-2013$ & 2005 & $2009-2010$ & 2008 & $2013^{s}$ & 2006 & 2012 & 2008 & 2012 \\
\hline & \multicolumn{2}{|c|}{$(4-17 y)$} & \multicolumn{2}{|c|}{$(11 y)$} & \multicolumn{2}{|c|}{$(7-9 y)$} & \multicolumn{2}{|c|}{$(6,9,12,14 y)$} & \multicolumn{2}{|c|}{$(8-9 y)$} \\
\hline OW/OB (\%) & 17.7 & 16.4 & 18.2 & 16.8 & 16.6 & 17.0 & 21.1 & 21.0 & 15.0 & 15.8 \\
\hline $\mathrm{OB}(\%)$ & 3.2 & 2.8 & 2.6 & 2.8 & 3.0 & 2.1 & 4.8 & 4.7 & 3.3 & 3.5 \\
\hline
\end{tabular}

\footnotetext{
Note: $\quad$ *Measured data.

\#Only data from the Reykjavík area.

${ }^{\$}$ Only data from Västa Götaland.
} 
The prevalence of $O W / O B$ and $O B$ were comparable between other national estimates and NORMO data, except for OW/OB in Denmark. Other national estimates display the highest prevalence of OW/OB among children in Iceland. This is in agreement with NORMO data. The prevalence was similar in the four other countries according to other national estimates. This is in contrast to NORMO data revealing a lower prevalence of OW/OB among children in Denmark than among childen in Sweden and Norway.

Other national estimates show in line with NORMO data no change in the OW/OB and $\mathrm{OB}$ prevalence among children in the Nordic countries during the last decade. This is also supported by results from the WHO Health Behaviour in School-aged Children (HBSC) study (Currie et al. 2008; Currie et al. 2012).

In summary, the prevalence and the development of OW/OB and $\mathrm{OB}$ according to other national estimates from the Nordic countries were in line with NORMO data, except for OW/OB prevalence in Denmark. Other national estimates support NORMO data in that the $O W / O B$ and $O B$ prevalence among children in the Nordic countries is on a plateau.

\subsubsection{Evaluation of Goal 2011 and Vision 2021 in the Nordic Plan of Action among children}

Goal 2011 and Vision 2021 in the Nordic Plan of Action for overweight and obesity were almost similar for children and adults (Appendix C).

Goal 2011 has been fulfilled among children because the prevalence of OW/OB and OB did not change between 2011 and 2014 in the Nordic region. The next step will be to reduce the prevalence of $\mathrm{OW} / \mathrm{OB}$ and $\mathrm{OB}$ among children in the Nordic countries in order to fulfill Vision 2021. Girls may be especially relevant to target because the prevalence of OW/OB increased between 2011 and 2014.

Vision 2021 aims to reduce the OW/OB and OB prevalence with at least 50\% from the prevalence at year 2006 (Table 74).

Table 74: Target prevalence of OW/OB and OB among children according to Vision 2021 in the Nordic Plan of Action. Target prevalence corresponds to a $50 \%$ reduction from the prevalence at year 2006

\begin{tabular}{lrrrrr} 
& Denmark & Finland & Sweden & Iceland & Norway \\
\hline OW/OB (\%) & 8.9 & 9.1 & 8.3 & 10.6 & 7.5 \\
OB (\%) & 1.6 & 1.3 & 1.5 & 2.4 & 1.7 \\
\hline
\end{tabular}


In 2014, the prevalence of OW/OB and OB was $12-20 \%$ and $2-4 \%$, respectively, among children in the five Nordic countries and the target prevalence in Vision 2021 is 8-11\% for OW/OB and $1-2 \%$ for OB. Hence, the current prevalence of OW/OB and OB should almost be halved in a seven year period to reach the target prevalence in Vision 2021. This may be difficult, but it will probably be easier to fulfill Vision 2021 for OB than for OW/OB among children in the Nordic region. Denmark is close to meet the target prevalence of OW/OB in Vision 2021 while Finland, Norway and Sweden have a longer way to go, and Iceland is far away.

\subsubsection{Social inequality}

Goal 2011 may be regarded as fulfilled because the social difference in OW/OB and OB decreased among children in the Nordic region from 2011 to 2014.

Vision 2021 states that the social difference in OW/OB should decrease and be at most $20 \%$. The social difference in OW/OB decreased from $98 \%$ in 2011 to $19 \%$ in 2014 in the Nordic region. Currently, Vision 2021 may be regarded as fulfilled. However, even if the social difference in OW/OB has become much smaller, social inequality in OW/OB and OB persist among children in the Nordic region. Furthermore, Vision 2021 needs to be evaluated with the ambition of achieving better health in all social groups and not just decrease social inequality in OW/OB as mentioned previously. 


\section{Smoking}

Smoking is a major risk factor for many of the leading causes of premature death. Smoking increases the risk of cardiovascular diseases, stroke, lung cancer and other types of cancers. Smoking also causes a number of other chronic conditions and diseases, such as obstructive pulmonary disease, that have a substantial impact on population health and wellbeing (U.S. Department of Health and Human Services, 2014). Thus, smoking poses a major threat to health in the Nordic region.

In this chapter, population levels of smoking, i.e. the proportions of smokers and daily smokers, are reported among adults in the Nordic countries. Smoking was assessed for the first time in NORMO 2014 why it is only possible to examine population levels. The proportion of smokers is provided by summing up daily and occasional smokers. Other national estimates are used to compare the population levels and to report the most recent development of smoking in the Nordic countries.

Data on the population levels of smoking and daily smoking according to age and education in each of the five Nordic countries are commented in this chapter, but data are not tabulated.

Population levels of users of smokeless tobacco (snuff) and daily users of smokeless tobacco (snuff) are shown in Table 104 in appendix D, but data will not be commented and were not analysed statistically. Smoking was not part of the Nordic Plan of Action in 2006 (Nordic Council of Ministers 2006) why no goals and visions have been formulated for smoking in the Nordic countries.

When comparing smoking behaviour between the Nordic countries, only countries where it is possible to identify one or more countries with the highest and/or lowest proportions of smokers and daily smokers will be commented in the key findings below.

\subsection{Key findings}

- In 2014, one in five adults were smokers (i.e. daily or occasionally smokers) and one in seven were daily smokers in the Nordic region. The proportion of smokers was higher in men than in women (21.8\% vs. $19.9 \%)$, while no gender difference was seen for daily smokers. The lowest proportion of smokers and daily smokers 
was observed in Sweden, while the highest proportion of daily smokers was observed in Denmark.

- In the Nordic region, the proportion of smokers was higher in men than in women, while no gender difference was found for daily smokers.

- The proportion of smokers was higher in 18-24- and 25-44-y-olds than in 45-65$y$-olds, whereas the proportion of daily smokers was higher in $45-65$-y-olds than in 18-24-y-olds.

- An inverse social gradient was found in smoking and daily smoking, i.e. the proportions of smokers and daily smokers decreased with increasing level of education. The social differences in smoking and daily smoking were large in the Nordic countries in 2014.

- According to other national estimates the proportions of smokers and daily smokers have been declining in all the Nordic countries during the last decade.

\subsubsection{Conclusions}

The proportions of smokers and daily smokers were $21 \%$ and $14.0 \%$, respectively, in the Nordic region. In the Nordic countries, the proportion of smokers varied between 15$25 \%$ and the proportion of daily smokers between $9-21 \%$. The proportion of daily smokers was higher in Denmark than in the other Nordic countries. Large social differences in smoking were found in all the Nordic countries.

\subsection{Results}

\subsubsection{Adults}

In 2014, one in five adults were smokers, i.e. daily or occasionally smokers, and one in seven were daily smokers in the Nordic region (Table 75). Ranking of the proportion of smokers and daily smokers in the five Nordic countries is shown in Table 76. The proportion of smokers was lower in Sweden than in the other Nordic countries and higher in Denmark than in Iceland. Moreover, the proportion of daily smokers differed between all the Nordic countries, except for Iceland and Norway where no difference was found. 
Table 75: Proportion of smokers $(95 \% \mathrm{Cl})$ among adults in the Nordic countries. NORMO 2014

\begin{tabular}{|c|c|c|c|c|c|c|}
\hline & Denmark & Finland & Sweden & Iceland & Norway & Nordic region@ \\
\hline & $2014(n=2,006)$ & $2014(n=1,712)$ & $2014(n=1,798)$ & $2014(n=2,006)$ & $2014(n=1,057)$ & $2014(n=8,579)$ \\
\hline \multirow[t]{2}{*}{ Smokers (\%) } & $25 \cdot 3$ & 24.4 & 15.4 & 22.0 & 22.2 & 20.8 \\
\hline & $(23.4 ; 27.2)$ & $(22.4 ; 26.5)$ & $(13.7 ; 17.0)$ & $(20.2 ; 23.8)$ & $(19.7 ; 24.7)$ & $(20.0 ; 21.7)$ \\
\hline Daily smokers" & 21.3 & 17.8 & 9.4 & 15.2 & 14.2 & 14.8 \\
\hline$(\%)$ & $(19.5 ; 23.1)$ & $(16.0 ; 19.6)$ & $(8.1 ; 10.8)$ & $(13.6 ; 16.7)$ & $(12.1 ; 16.3)$ & $(14.0 ; 15.5)$ \\
\hline
\end{tabular}

Note: @Weighted according to population size in the five Nordic countries.

"Subgroup of smokers.

Table 76: Ranking from lowest to highest proportion of smokers and daily smokers among adults in the five Nordic countries. NORMO 2014

\begin{tabular}{lclc} 
& Smokers (\%) & & Daily smokers (\%) \\
Sweden & 15.4 & Sweden & 9.4 \\
Iceland & 22.0 & Norway & 14.2 \\
Norway & 22.2 & Iceland & 15.2 \\
Finland & 24.4 & Finland & 17.8 \\
Denmark & 25.3 & Denmark & 21.3 \\
\hline
\end{tabular}

\subsubsection{Gender}

In the Nordic region, the proportion of smokers was higher in men than in women (21.8\% vs. $19.9 \%)$, while the proportion of daily smokers was similar between genders (Table 77 and 78). The proportion of smokers and daily smokers was higher in men than in women in Finland. It was the other way around in Sweden where the proportion of daily smokers was higher in women than in men.

Table 77: Proportion of smokers (95\% Cl) among men in the Nordic countries. NORMO 2014

\begin{tabular}{|c|c|c|c|c|c|c|}
\hline & $\begin{array}{r}\text { Denmark } \\
2014(n=976)\end{array}$ & $\begin{array}{r}\text { Finland } \\
2014(n=753)\end{array}$ & $\begin{array}{r}\text { Sweden } \\
2014(n=826)\end{array}$ & $\begin{array}{r}\text { Iceland } \\
2014(n=890)\end{array}$ & $\begin{array}{r}\text { Norway } \\
2014(n=484)\end{array}$ & $\begin{array}{l}\text { Nordic region } \\
2014(n=3,929)\end{array}$ \\
\hline Smokers (\%) & $\begin{array}{r}26.6 \\
(23.9 ; 29.4)\end{array}$ & $\begin{array}{r}30.6 \\
(27.3333 .9)\end{array}$ & $\begin{array}{r}14.3 \\
(11.9 ; 16.7)\end{array}$ & $\begin{array}{r}21.9 \\
(19.2 ; 24.7)\end{array}$ & $\begin{array}{r}21.1 \\
(17.4 ; 24.7)\end{array}$ & $\begin{array}{r}21.8 \\
(20.5 ; 23.0)\end{array}$ \\
\hline Daily smokers" (\%) & $\begin{array}{r}22.5 \\
(19.9 ; 25.1)\end{array}$ & $\begin{array}{r}21.5 \\
(18.6 ; 24.5)\end{array}$ & $\begin{array}{r}7.5 \\
(5.7 ; 9.3)\end{array}$ & $\begin{array}{r}15.3 \\
(13.0 ; 17.7)\end{array}$ & $\begin{array}{r}12.2 \\
(9.3 ; 15.1)\end{array}$ & $\begin{array}{r}14.7 \\
(13.6 ; 15.8)\end{array}$ \\
\hline
\end{tabular}

Note: @Weighted according to population size in the five Nordic countries.

"Subgroup of smokers. 
Table 78: Proportion of smokers ( $95 \% \mathrm{Cl}$ ) among women in the Nordic countries. NORMO 2014

\begin{tabular}{|c|c|c|c|c|c|c|}
\hline & Denmark & Finland & Sweden & Iceland & Norway & Nordic region@ \\
\hline & $2014(n=1,029)$ & $2014(n=959)$ & $2014(n=972)$ & $2014(n=1,116)$ & $2014(n=573)$ & $2014(n=4,649)$ \\
\hline Smokers (\%) & $\begin{array}{r}23.9 \\
(21.3 ; 26.5)\end{array}$ & $\begin{array}{r}18.7 \\
(16.2 ; 21.2)\end{array}$ & $\begin{array}{r}16.5 \\
(14.1 ; 18.8)\end{array}$ & $\begin{array}{r}22.1 \\
(19.7 ; 24.6)\end{array}$ & $\begin{array}{r}23.2 \\
(19.8 ; 26.7)\end{array}$ & $\begin{array}{r}19.9 \\
(18.7 ; 21.0)\end{array}$ \\
\hline Daily smokers ${ }^{\#}(\%)$ & $\begin{array}{r}20.2 \\
(17.7 ; 22.6)\end{array}$ & $\begin{array}{r}14.4 \\
(12.2 ; 16.6)\end{array}$ & $\begin{array}{r}11.4 \\
(9.4 ; 13.4)\end{array}$ & $\begin{array}{r}15.0 \\
(12.9 ; 17.1)\end{array}$ & $\begin{array}{r}16.2 \\
(13.2 ; 19.2)\end{array}$ & $\begin{array}{r}14.8 \\
(13.8 ; 15.9)\end{array}$ \\
\hline
\end{tabular}

Note: @Weighted according to population size in the five Nordic countries.

\#Subgroup of smokers.

\subsubsection{Age}

In the Nordic region, the proportion of smokers was higher in 18-24- and 25-44-y-olds than in $45-65$-y-olds, whereas the proportion of daily smokers was higher in $45-65-y-$ olds than in 18-24-y-olds (Table 79). Analyses in each of the five Nordic countries showed that the proportion of smokers was higher in $18-24-y$-olds than in $25-44$ - and 45-65-y-olds in Sweden and Iceland, while the proportion of smokers was higher in 2544-y-olds than in 45-65-y-olds in Finland. Moreover, the proportion of daily smokers was higher in $18-24-y$-olds than in $25^{-44-}$ and $45-65$-y-olds in Iceland, while the proportion of daily smokers was higher in $45-65$-y-olds than in $18-24-y$-olds in Norway.

Table 79: Proportion of smokers $(95 \% \mathrm{Cl})$ among adults according to age in the Nordic region. NORMO 2014

\begin{tabular}{|c|c|c|c|}
\hline & \multicolumn{3}{|c|}{ Nordic region@ } \\
\hline & $\begin{array}{r}18-24 y \\
2014(n=846)\end{array}$ & $\begin{array}{r}25-44 y \\
2014(n=3,075)\end{array}$ & $\begin{array}{r}45-65 y \\
2014(n=4,658)\end{array}$ \\
\hline Smokers (\%) & $\begin{array}{r}23.3 \\
(20.5 ; 26.0)\end{array}$ & $\begin{array}{r}21.3 \\
(19.9 ; 22.8)\end{array}$ & $\begin{array}{r}19.3 \\
(18.1 ; 20.4)\end{array}$ \\
\hline Daily smokers" (\%) & $\begin{array}{r}12.7 \\
(10.5 ; 14.9)\end{array}$ & $\begin{array}{r}14.2 \\
(13.0 ; 15.4)\end{array}$ & $\begin{array}{r}15.8 \\
(14.8 ; 16.9)\end{array}$ \\
\hline
\end{tabular}

Note: @Weighted according to population size in the five Nordic countries.

\#Subgroup of smokers. 


\subsubsection{Education}

An inverse social gradient was found in smoking in the Nordic region and in each of the five Nordic countries, i.e. the proportions of smokers and daily smokers decreased with increasing level of education (Table 80). Only in Norway no difference was found in the proportion of smokers between the medium and high educated. The social differences in smoking and daily smoking were large in the Nordic region and in each of the five Nordic countries.

Table 80: Proportion of smokers $(95 \% \mathrm{Cl})$ among adults according to education in the Nordic region. NORMO 2014

\begin{tabular}{lrrr} 
& & \multicolumn{1}{c}{ Nordic region@ } & \\
& Low & Medium & High \\
& 2014 & 2014 & 2014 \\
& $(n=1,064)$ & $(n=3,135)$ & $(n=4,364)$
\end{tabular}

Relative difference total smokers* $(\%)$

Relative difference daily smokers* $(\%)$

Note: @Weighted according to population size in the five Nordic countries. \#Subgroup of smokers.

${ }^{*}$ Relative difference $(\%)=[($ value High education group - value Low education group $) /$ value High education group]*100.

\subsection{Discussion}

The proportions of smokers and daily smokers were $20.8 \%$ and $14.8 \%$, respectively, among adults in the Nordic region. This means that one in five adults or 3.4 million people in the Nordic countries are daily or occassional smokers.

The proportion of smokers was similar in the Nordic countries, except in Sweden where the lowest proportion was found. One of the reasons for the low proportion of smokers in Sweden, particularly in men, may be the common use of smokeless tobacco (snuff) as shown in Table 104 in appendix D. In the Nordic region, smoking was most prevalent among men, $18-24$ - and $25-44-y$-olds and the low educated.

Because of the very large social differences in smoking behaviour in all the Nordic countries, implementation of smoking cessation with high reach among the low educated is needed. Policy making, prevention and smoking cessation are needed actions 
to decrease the proportion of smokers in the Nordic countries. This may have a large effect on the health of the population in the Nordic region.

\subsubsection{Comparison with other national estimates from the Nordic countries among adults}

The population levels and the development of smoking among adults in the Nordic countries according to other national estimates are shown in Table 81. Other national estimates are useful to compare the population levels of smoking with NORMO data and to show the most recent development of smoking in the Nordic countries. Other national estimates display the development of smoking from 2007 to 2014 .

When comparing other national estimates with NORMO data, population levels and rankings of the proportion of smokers in the Nordic countries differ. However, the ranking of Denmark as the country with the highest proportion of daily smokers and Sweden as the country with the lowest proportion of daily smokers was similar for other national estimates and NORMO data. Other national estimates showed lower proportions of smokers and daily smokers in Denmark, Finland and Iceland than NORMO data, while the proportion of smokers was higher in Sweden. Similar estimates were seen for smoking and daily smoking in Norway and for the proportion of daily smokers in Sweden. It is not clear what the reason may be for the differences mentioned above.

Other national estimates show that the proportions of smokers and daily smokers are decreasing in all the Nordic countries. The proportion of smokers has decreased two to seven percentage points between 2007 and 2014 in the five Nordic countries according to other national estimates. The decrease in smoking behaviour was primarily due to a decrease in the proportion of daily smokers.

In summary, population levels of smoking in the Nordic countries according to other national estimates are not in line with NORMO data.

Table 81: Population levels and the development of smoking among adults in the Nordic countries according to other national estimates

\begin{tabular}{|c|c|c|c|c|c|c|c|c|c|c|}
\hline & \multicolumn{2}{|c|}{ Denmark } & \multicolumn{2}{|c|}{ Finland } & \multicolumn{2}{|c|}{ Sweden } & \multicolumn{2}{|c|}{ Iceland } & \multicolumn{2}{|c|}{ Norway } \\
\hline & $\begin{array}{r}2010 \\
\quad(16-\end{array}$ & 2013 & $\begin{array}{l}2011 \\
(15-\end{array}$ & $\begin{array}{l}2014 \\
\text { y) }\end{array}$ & $\begin{array}{r}2011 \\
(16-\end{array}$ & $\begin{array}{l}2014 \\
\text { y) }\end{array}$ & $\begin{array}{r}2007 \\
\quad(18\end{array}$ & 2012 & $\begin{array}{c}2010 \\
\quad(16-\end{array}$ & $\begin{array}{l}2013 \\
\text { y) }\end{array}$ \\
\hline Total smokers (\%) & $25 \cdot 3$ & 21.6 & 24.9 & 21.9 & 22.0 & 20.0 & 23.7 & 17.2 & 28.8 & 23.2 \\
\hline Daily smokers (\%) & 20.9 & 17.0 & 16.4 & 13.8 & 11.0 & 10.0 & 18.2 & 12.4 & 18.9 & 14.4 \\
\hline Occasional smokers (\%) & 4.4 & 4.6 & 8.5 & 8.1 & 11.0 & 10.0 & 5.5 & 4.8 & 9.9 & 8.8 \\
\hline
\end{tabular}




\section{Alcohol}

Alcohol consumption is associated with mostly negative health effects such as increased risk of certain types of cancers and injuries and tends to have a negative effect on diet quality (NNR 2012, WHO 2011). Based on the overall evidence, it is recommended to limit alcohol consumption and avoid binge drinking.

In this chapter, population levels of alcohol consumption, i.e. mean total alcohol consumption per week and the proportion of binge drinkers at least once during the last month, ${ }^{8}$ are reported among adults in the Nordic countries. Alcohol consumption was assessed for the first time in NORMO 2014 why it is only possible to examine the population levels. Other national estimates are used to compare the population levels and describe the most recent development of alcohol consumption in the Nordic countries. Data on the population levels of alcohol consumption according to age and education in each of the five Nordic countries are commented in this chapter, but data are not tabulated.

Alcohol was not part of the Nordic Plan of Action in 2006 (Nordic Council of Ministers) why no goals and visions have been formulated for alcohol consumption in the Nordic countries.

When comparing alcohol consumption between the Nordic countries, only countries where it is possible to identify one or more countries with the highest and/or lowest mean total alcohol consumption and proportion of binge drinkers will be commented in the key findings below.

\subsection{Key findings}

\subsubsection{Adults}

- In 2014, mean total alcohol consumption was 1.7 times/week and $45 \%$ were binge drinkers at least once during the last month among adults in the Nordic region. Mean total alcohol consumption and the proportion of binge drinkers were higher

\footnotetext{
${ }^{8}$ The proportion of binge drinkers at least once during the last month will be referred as just the proportion of binge drinkers to keep the terms short.
} 
in men than in women (2.2 vs. 1.3 times/week and $55.7 \%$ vs. $34.2 \%$ ). The lowest mean total alcohol consumption was seen in Iceland and the highest in Denmark. The lowest proportion of binge drinkers was observed in Sweden and Iceland, and the highest in Norway.

- In the Nordic region, mean total alcohol consumption and the proportion of binge drinkers were higher in men than in women (2.2 vs. 1.3 times/week and $55.7 \%$ vs. $34.2 \%)$.

- Mean total alcohol consumption was higher in 45-65-y-olds than in 18-24- and 25-44-y-olds, whereas the proportion of binge drinkers decreased across age groups.

- Mean total alcohol consumption was higher among the high educated than among the medium and low educated, while the proportion of binge drinkers was higher among the medium educated than among the high educated. In the Nordic region, the social difference in alcohol consumption was moderate and just small in binge drinking.

- According to other national estimates the proportions exceeding the weekly alcohol limits and binge drinkers did not change or decreased in the Nordic countries over the last decade.

\subsubsection{Conclusions}

Mean total alcohol consumption was 1.7 times/week and the proportion of binge drinkers was $45 \%$ among adults in the Nordic region. In the Nordic countries, mean total alcohol consumption varied between 1.2-2.5 times/week and the proportion of binge drinkers between $39-52 \%$ in 2014. The highest mean total alcohol consumption was seen in Denmark and the highest proportion of binge drinkers in Norway. The social difference in alcohol consumption was moderate and just small in binge drinking in the Nordic region.

\subsection{Results}

\subsubsection{Adults}

In 2014, mean total alcohol consumption was 1.7 times/week and $45 \%$ were binge drinkers at least once during the last month among adults in the Nordic region (Table 82). Ranking of the alcohol consumption and the proportion of binge drinkers in the five Nordic countries is shown in Table 83. The alcohol consumption was higher in Denmark 
than in the other Nordic countries and lower in Iceland than in the other countries. The proportion of binge drinkers was higher in Norway than in the other Nordic countries and lower in Iceland and Sweden than in Finland and Denmark.

Table 82: Mean total alcohol consumption and the proportion of binge drinkers ( $95 \% \mathrm{Cl}$ ) among adults in the Nordic countries. NORMO 2014

\begin{tabular}{|c|c|c|c|c|c|c|}
\hline & Denmark & Finland & Sweden & Iceland & Norway & Nordic region@ \\
\hline & $\begin{array}{r}2014 \\
(n=2,007)\end{array}$ & $\begin{array}{r}2014 \\
(n=1,711)\end{array}$ & $\begin{array}{r}2014 \\
(n=1,799)\end{array}$ & $\begin{array}{r}2014 \\
(n=2,004)\end{array}$ & $\begin{array}{r}2014 \\
(n=1,056)\end{array}$ & $\begin{array}{r}2014 \\
(n=8,577)\end{array}$ \\
\hline $\begin{array}{l}\text { Total alcohol consump- } \\
\text { tion (freq./week) }\end{array}$ & $\begin{array}{r}2.5 \\
(2.3 ; 2.6)\end{array}$ & $\begin{array}{r}1.5 \\
(1.4 ; 1.6)\end{array}$ & $\begin{array}{r}1.5 \\
(1.4 ; 1.6)\end{array}$ & $\begin{array}{r}1.2 \\
(1.1 ; 1.3)\end{array}$ & $\begin{array}{r}1.6 \\
(1.4 ; 1.8)\end{array}$ & $\begin{array}{r}1.7 \\
(1.7 ; 1.8)\end{array}$ \\
\hline $\begin{array}{l}\text { Binge drinking last } \\
\text { month (\%) }\end{array}$ & $\begin{array}{r}46.3 \\
(44.1 ; 48.5)\end{array}$ & $\begin{array}{r}44.5 \\
(42.1 ; 46.8)\end{array}$ & $\begin{array}{r}40.9 \\
(38.5 ; 43.3)\end{array}$ & $\begin{array}{r}39.1 \\
(37.0 ; 41.3)\end{array}$ & $\begin{array}{r}51.5 \\
(48.5 ; 54.6)\end{array}$ & $\begin{array}{r}45.0 \\
(43.9 ; 46.1)\end{array}$ \\
\hline
\end{tabular}

Note: @Weighted according to population size in the five Nordic countries.

Table 83: Ranking from lowest to highest mean total alcohol consumption and the proportion of binge drinkers among adults in the five Nordic countries. ${ }^{9}$ NORMO 2014 Total alcohol consumption (freq./week) Binge drinking last month (\%)

$\begin{array}{llll}\text { Iceland } & 1.2 & \text { Iceland } & 39.1 \\ \text { Sweden } & 1.5 & \text { Sweden } & 40.9 \\ \text { Finland } & 1.5 & \text { Finland } & 44.5 \\ \text { Norway } & 1.6 & \text { Denmark } & 46.3 \\ \text { Denmark } & 2.5 & \text { Norway } & 51.5\end{array}$

\subsubsection{Gender}

In the Nordic region, the alcohol consumption and the proportion of binge drinkers were higher in men than in women (2.2 vs 1.3 times/week and $55.7 \%$ vs. $34.2 \%$ ). This gender difference in alcohol behaviour was found in all the Nordic countries (Table 84 and 85 ).

$9 \geq 5$ units of alcohol per drinking occasion at least once during the last month 
Table 84: Mean total alcohol consumption and the proportion of binge drinkers $(95 \% \mathrm{Cl})$ among men in the Nordic countries. NORMO 2014

\begin{tabular}{|c|c|c|c|c|c|c|}
\hline & Denmark & Finland & Sweden & Iceland & Norway & Nordic region \\
\hline & $\begin{array}{r}2014 \\
(n=976)\end{array}$ & $\begin{array}{r}2014 \\
(n=753)\end{array}$ & $\begin{array}{r}2014 \\
(n=826)\end{array}$ & $\begin{array}{r}2014 \\
(n=888)\end{array}$ & $\begin{array}{r}2014 \\
(n=483)\end{array}$ & $\begin{array}{r}2014 \\
(n=3,926)\end{array}$ \\
\hline $\begin{array}{l}\text { Total alcohol consump- } \\
\text { tion (freq./week) }\end{array}$ & $\begin{array}{r}3.2 \\
(2.9 ; 3.5)\end{array}$ & $\begin{array}{r}2.0 \\
(1.8 ; 2.2)\end{array}$ & $\begin{array}{r}1.8 \\
(1.7 ; 1.9)\end{array}$ & $\begin{array}{r}1.5 \\
(1.3 ; 1.6)\end{array}$ & $\begin{array}{r}1.9 \\
(1.5 ; 2.3)\end{array}$ & $\begin{array}{r}2.2 \\
(2.1 ; 2.3)\end{array}$ \\
\hline $\begin{array}{l}\text { Binge drinking last } \\
\text { month (\%) }\end{array}$ & $\begin{array}{r}55.5 \\
(52.4 ; 58.6)\end{array}$ & $\begin{array}{r}58.2 \\
(54.7 ; 61.8)\end{array}$ & $\begin{array}{r}53.8 \\
(50.3 ; 57.3)\end{array}$ & $\begin{array}{r}46.0 \\
(42.7 ; 49.3)\end{array}$ & $\begin{array}{r}57.2 \\
(52.8 ; 61.7)\end{array}$ & $\begin{array}{r}55.7 \\
(54.1 ; 57 \cdot 3)\end{array}$ \\
\hline
\end{tabular}

Table 85: Mean total alcohol consumption and the proportion of binge drinkers $(95 \% \mathrm{Cl})$ among women in the Nordic countries. NORMO 2014

\begin{tabular}{|c|c|c|c|c|c|c|}
\hline & Denmark & Finland & Sweden & Iceland & Norway & Nordic region@ \\
\hline & $\begin{array}{r}2014 \\
(n=1,030)\end{array}$ & $\begin{array}{r}2014 \\
\left(n=95^{8}\right)\end{array}$ & $\begin{array}{r}2014 \\
(n=973)\end{array}$ & $\begin{array}{r}2014 \\
(n=1,116)\end{array}$ & $\begin{array}{r}2014 \\
(n=573)\end{array}$ & $\begin{array}{r}2014 \\
(n=4,650)\end{array}$ \\
\hline $\begin{array}{l}\text { Total alcohol consump- } \\
\text { tion (freq./week) }\end{array}$ & $\begin{array}{r}1.7 \\
(1.6 ; 1.9)\end{array}$ & $\begin{array}{r}1.1 \\
(1.0 ; 1.2)\end{array}$ & $\begin{array}{r}1.1 \\
(1.1 ; 1.2)\end{array}$ & $\begin{array}{r}0.9 \\
(0.8 ; 1.0)\end{array}$ & $\begin{array}{r}1.3 \\
(1.1 ; 1.4)\end{array}$ & $\begin{array}{r}1.3 \\
(1.2 ; 1.3)\end{array}$ \\
\hline $\begin{array}{l}\text { Binge drinking last } \\
\text { month (\%) }\end{array}$ & $\begin{array}{r}36.9 \\
(34.0 ; 39.9)\end{array}$ & $\begin{array}{r}31.5 \\
(28.5 ; 34.5)\end{array}$ & $\begin{array}{r}27.2 \\
(24.3 ; 30.2)\end{array}$ & $\begin{array}{r}32.3 \\
(29.6 ; 35.1)\end{array}$ & $\begin{array}{r}45.9 \\
(41.8 ; 50.0)\end{array}$ & $\begin{array}{r}34.2 \\
(32.8 ; 35.6)\end{array}$ \\
\hline
\end{tabular}

Note: @Weighted according to population size in the five Nordic countries.

\subsubsection{Age}

In the Nordic Region, alcohol consumption was higher in 45-65-y-olds than in 18-24and 25-44-y-olds (Table 86). This was found in all the Nordic countries, except in Norway where alcohol consumption was higher in 45-65-y-olds than in $25-44$ - $y$-olds.

An inverse age gradient was found in binge drinking in the Nordic region, i.e. the proportion of binge drinkers decreased across age groups. This inverse age gradient was found in all the Nordic countries, except in Norway where the proportion of binge drinkers was higher in 18-24- and 25-45-y-olds than in 45-65-y-olds. 
Table 86: Mean total alcohol consumption and the proportion of binge drinkers ( $95 \% \mathrm{Cl}$ ) among adults according to age in the Nordic region. NORMO 2014

\begin{tabular}{|c|c|c|c|}
\hline & \multicolumn{2}{|c|}{ Nordic region@ } & \multirow[b]{2}{*}{$\begin{array}{r}45-65 y \\
2014(n=4,655)\end{array}$} \\
\hline & $\begin{array}{r}18-24 Y \\
2014(n=846)\end{array}$ & $\begin{array}{r}25-44 y \\
2014(n=3,076)\end{array}$ & \\
\hline Total alcohol consumption (freq./week) & $\begin{array}{r}1.4 \\
(1.3 ; 1.5)\end{array}$ & $\begin{array}{r}1.4 \\
(1.3 ; 1.4)\end{array}$ & $\begin{array}{r}2.2 \\
(2.1 ; 2.3)\end{array}$ \\
\hline Binge drinking last month (\%) & $\begin{array}{r}64.3 \\
(61.1 ; 67.5)\end{array}$ & $\begin{array}{r}48.0 \\
(46.2 ; 49.8)\end{array}$ & $\begin{array}{r}35.5 \\
(34.1 ; 37.0)\end{array}$ \\
\hline
\end{tabular}

Note: ${ }^{\circledR}$ Weighted according to population size in the five Nordic countries.

\subsubsection{Education}

Alcohol consumption was higher among the high educated than among the medium and low educated in the Nordic region (Table 87). Analyses in each of the five Nordic countries showed that alcohol consumption was higher among the high educated than among the medium educated in Finland and higher among the medium and high educated than among the low educated in Iceland.

Table 87: Mean total alcohol consumption and the proportion of binge drinkers $(95 \% \mathrm{Cl}$ ) among adults according to education in the Nordic region. NORMO 2014

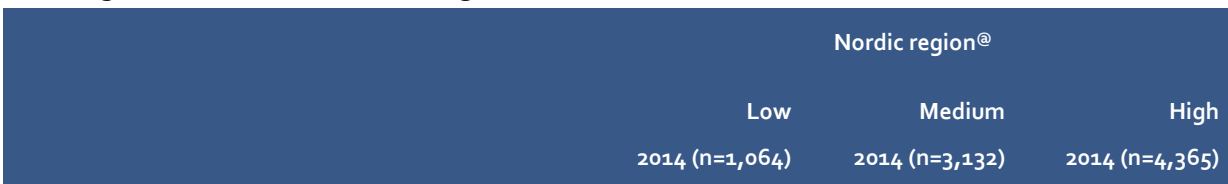

Total alcohol consumption (freq./week)

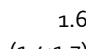

$.4 ; 1.7)$

$$
\begin{array}{r}
1.7 \\
(1.6 ; 1.8)
\end{array}
$$

Binge drinking last month (\%)

$\begin{array}{rrr}45.4 & 46.2 & 43.2 \\ (41.8 ; 49.0) & (44.4 ; 47.9) & (41.8 ; 44.7)\end{array}$

Relative difference total alcohol consumption\# (\%)

$\begin{array}{lll}- & - & 16\end{array}$

Relative difference binge drinking last month\# (\%)

$-$

Note: @Weighted according to population size in the five Nordic countries.

${ }^{\#}$ Relative difference $(\%)=[($ value High education group - value Low education group)/value High education group]*100.

In the Nordic region and in Sweden, the proportion of binge drinkers was higher among the medium educated than among the high educated. In Denmark, the proportion of 
binge drinkers was higher among the medium and high educated than among the low educated, while the proportion of binge drinkers was higher among the low and medium educated than among the high educated in Finland. Finally, the proportion of binge drinkers was higher among the low educated than among the medium educated in Norway.

The social difference in alcohol consumption was moderate in the Nordic region and in all the Nordic countries, except in Iceland with a large difference. A small social difference in binge drinking was found when analysing the Nordic region, while large social differences were observed in Denmark, Finland and Norway, a moderate difference in Sweden and just a small difference in Iceland.

\subsection{Discussion}

A mean total alcohol consumption of 1.7 times per week was found among adults in the Nordic region. Large differences were found in alcohol consumption between the Nordic countries. The alcohol consumption was two times higher in Denmark than in Iceland. No state-owned retail alcohol monopoly exists in Denmark in contrast to the other Nordic countries (Hallberg and Österberg 2014). In addition, Denmark is the country with the lowest age limit for purchasing alcohol in retail sale and the lowest taxation and prices of alcoholic beverages.

The highest alcohol consumption was seen among men, 45-65-y-olds and the high educated which make these groups relevant to target when planning public health initiatives for the general population.

Binge drinking is a public health challenge in relation to alcohol consumption and the highest proportion of binge drinkers was found in Norway. Binge drinking was more prevalent among men than among women. The highest proportion of binge drinkers was observed in 18-24-y-olds.

The social differences in alcohol consumption were moderate in all the Nordic countries, except in Iceland with a large difference.

\subsubsection{Comparison with other national estimates from the Nordic countries}

Table 88 and 89 show the population levels and the development of alcohol consumption and the proportions exceeding weekly alcohol limits and binge drinkers in each of the five Nordic countries according to other national estimates. Other national estimates are useful to compare the population levels of alcohol consumption and binge drinking with 
NORMO data and to show the most recent development in the Nordic countries. Other national estimates display the development from 2005 to 2014.

Table 88: Population levels and the development of mean total alcohol consumption among adults in the Nordic countries according to other national estimates

\begin{tabular}{|c|c|c|c|c|c|c|c|c|c|}
\hline & \multicolumn{2}{|c|}{ Denmark } & \multicolumn{2}{|c|}{ Finland } & \multirow{2}{*}{$\begin{array}{r}\text { Sweden } \\
2010-2011 \\
(16-65 y)\end{array}$} & \multicolumn{2}{|c|}{ Iceland } & \multicolumn{2}{|c|}{ Norway } \\
\hline & $\begin{array}{r}2005^{-2008} \\
\left(15^{-}\right.\end{array}$ & $\begin{array}{l}2011-2013 \\
5 y)\end{array}$ & $\begin{array}{r}2011 \\
(25--\end{array}$ & $\begin{array}{l}2014 \\
\text { y) }\end{array}$ & & $\begin{array}{l}2007 \\
(18-\end{array}$ & 2012 & $\begin{array}{c}2012 \\
(16-79 y)\end{array}$ & 2014 \\
\hline $\begin{array}{l}\text { Total alcohol consump- } \\
\text { tion (frequency/week) }\end{array}$ & 3.0 & 2.7 & $\mathrm{~N} / \mathrm{A}$ & $N / A$ & 2.6 & 0.8 & 0.8 & N/A & $\mathrm{N} / \mathrm{A}$ \\
\hline
\end{tabular}

Note: N/A: Not available.

Table 89: Population levels and the development of the proportions exceeding weekly alcohol limits and binge drinkers among adults in the Nordic countries according to other national estimates

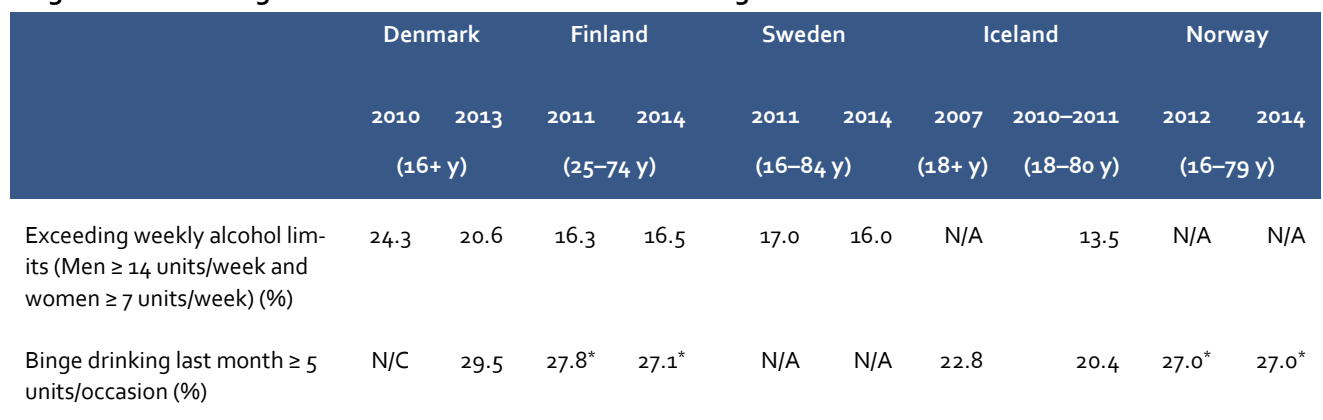

\section{Note: N/A: Not available. \\ $\mathrm{N} / \mathrm{C}$ : Not comparable. \\ ${ }^{*} \geq 6$ units/occasion.}

Few other national estimates on alcohol consumption and binge drinking were available for the Nordic countries.

Consumption of alcohol was similar for Denmark according to other national estimates and NORMO data, but other national estimates show higher alcohol consumption for Iceland and lower alcohol consumption for Sweden than NORMO data. The differences between other national estimates and NORMO data may be due to differences in participation rates, survey years, age groups and assessment method used. The ranking of Iceland as having the lowest consumption of alcohol and Denmark as having the highest was however the same for other national estimates and NORMO data. 
The population levels of binge drinkers are much lower in all the Nordic countries according to other national estimates compared with NORMO data. The large difference between other national estimates and NORMO data in the proportion of binge drinkers is mainly explained by definitions of binge drinking. Denmark may be used as an example to illustrate this because binge drinking was defined as binge drinking regularly each month according to other national estimates, while binge drinking in NORMO was defined as binge drinking at least once per month. Ranking of Iceland as the country with the lowest proportion of binge drinkers was however consistent between other national estimates and NORMO data.

Too few other national estimates were available on the mean total alcohol consumption to comment on the development over time. However, other national estimates show that the proportions exceeding the weekly alcohol limits and binge drinkers did not change or decreased over time in the Nordic countries.

In summary, the population levels of alcohol consumption and binge drinking in the Nordic countries according to other national estimates are not in agreement with NORMO data. This could be due to differences in participation rate, assessment methods and definitions (binge drinking) used. 


\section{Overview of results and discussion}

\subsection{The Nordic region}

The Nordic Monitoring System (NORMO) has now collected data for 2011 and 2014 among adults and children in the Nordic countries. The population levels and the development of diet, physical activity, sedentary behaviour and overweight/obesity and obesity in the Nordic region are shown in figure 9. A dietary quality score is used for describing the overall nutritional quality of the diet. The low scores, between $0-4$, is a measure of a diet, which is unhealthy in several parameters e.g. low in fruits and vegetables, whole grain bread, and/or fish and/or high in foods rich in saturated fat and added sugar. The low diet quality scores is used as a key variable and named "unhealthy diet". The proportions of inactive (not meeting physical activity recommendations) and with high recreational screen time $(>4 \mathrm{~h} /$ day) are used as indicators of physical activity and sedentary behaviour, respectively. The prevalence of overweight/obesity (OW/OB) and obesity (OB) is calculated on basis of BMI levels - Overweight/obesity: BMI $\geq 25$, Obesity: $\mathrm{BMI} \geq 30$.

Other national estimates from the Nordic countries for diet, physical activity, overweight, smoking and alcohol have been obtained to compare with the population levels and the development of the Nordic monitoring data. It is highly relevant to evaluate NORMO results against other estimates from national surveys, but this has highlighted the importance of measuring the population level and the development in the same survey years, age groups and using the same assessment method and definitions as has been done in NORMO. 
The overall picture shows that the proportion of adults with an unhealthy diet was more than $20 \%$ and had increased from 2011 to 2014 (Figure 9), while the proportion with a healthy diet decreased and only was 10\% in 2014 (Figure 1 in the chapter on diet). Around $1 / 3$ of all adults were inactive and three in ten spent more than 4 hours daily on recreational screen time. Furthermore, the increase in OB prevalence and in the proportions of highly inactive (almost no weekly moderate and vigorous intensity physical activity, Table 37 in the chapter on physical activity) and with sedentary behaviour (high recreational screen time) among adults are of concern. Finally, the prevalence of $\mathrm{OW} / \mathrm{OB}$ and $\mathrm{OB}$ among adults was high.

Among children in the Nordic region, there were no changes in diet, physical activity, sedentary behaviour and in the prevalence of OW/OB and OB between 2011 and 2014. Although it is encouraging that the same unfavorable development was not seen for children as for adults, the high population levels are of concern. Even though the proportion of children with an unhealthy diet was $15 \%$, the proportion with a healthy diet was only $10 \%$ as for adults (Figure 3 in the chapter on diet). The proportion of inactive children in the Nordic region was approx. $60 \%$ and therefore too high. Also the prevalence of OW/OB and OB was relative high; and a halt in development has previously been followed by a rise.

\subsection{Adults in the Nordic region}

In 2014, one in five adults had an unhealthy diet in the Nordic region. The proportion with an unhealthy diet was higher in men than in women ( $25 \% \mathrm{vs.} 18 \%$ ). The proportion with an unhealthy diet increased from $18 \%$ in 2011 to $22 \%$ in 2014 (Figure 9). The proportion increased more in men than in women. The proportion with an unhealthy diet decreased in 18-24-y-olds, but increased in $25-44^{-}$and $45-65-y$-olds and among the low and high educated. 
Figure 9: Population levels and the development in the proportions with an unhealthy diet, inactive and with high recreational screen time and the prevalence of $O W / O B$ and $O B$ among adults (18-65 years) in the Nordic region

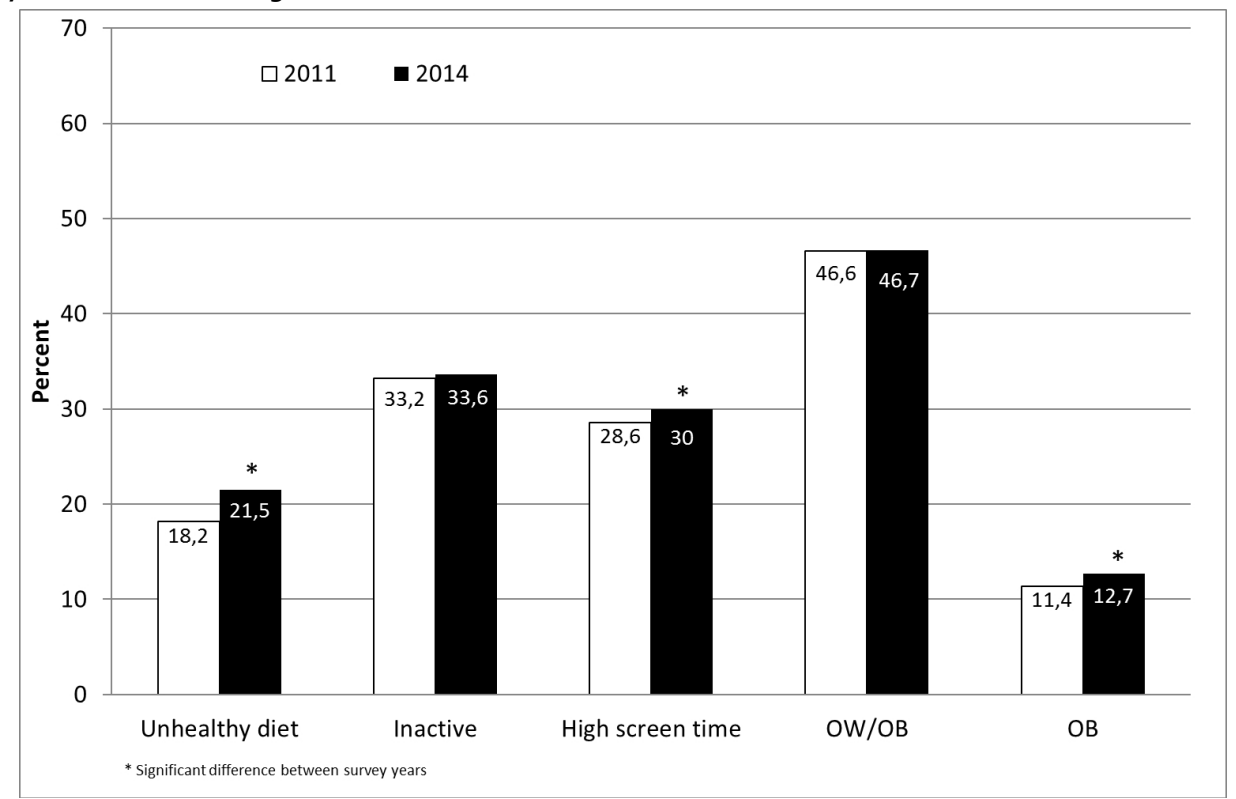

In 2014, one in three adults were physically inactive in the Nordic region. The proportion of inactive did not differ between genders. No change was observed in the proportion of inactive between 2011 and 2014 (Figure 9). This was also found when gender and education were analysed (Table 39, 40 and 45 in the chapter on physical activity). But the proportion of inactive increased in $18-24-y$-olds. Moreover, the proportion of highly inactive increased from $11 \%$ in 2011 to $12 \%$ in 2014 in the Nordic region (Table 37 in the chapter on physical activity). The proportion of highly inactive increased in women and 18-24-y-olds and among the medium and high educated. Between 2011 and 2014, the proportion of highly active increased from $13 \%$ to $14 \%$ in the Nordic region. The proportion of highly active increased in men and among the high educated. The increased proportions of both highly inactive and highly active adults indicate an increased polarisation in the physical activity pattern in the Nordic region.

With regard to sedentary behavior in 2014, three in ten adults spent more than four hours daily on recreational screen time in the Nordic region. The proportion with high recreational screen time was higher in men than in women (33\% vs. 27\%). The proportion with high recreational screen time increased from $29 \%$ in 2011 to $30 \%$ in 2014 (Figure 9), however no significant change was found in men or women. The proportion with 
high recreational screen time increased in $45-65-y$-olds and among the low educated from 2011 to 2014 .

In 2014, almost one in two adults were overweight or obese and one in eight were obese in the Nordic region. The prevalence of OW/OB and OB was higher in men than in women ( $56 \%$ vs. $37 \%$, and $14 \%$ vs. $11 \%$ ). No change was found in the prevalence of $O W / O B$ between 2011 and 2014. However, the prevalence of OB increased from $11 \%$ to $13 \%$ (Figure 9). This was mainly due to an $O B$ increase in women. The prevalence of $O B$ increased in $25-44^{-}$and $45-65-y$-olds and among the high educated, but decreased in 18-24-y-olds.

In 2014, one in five adults were smokers, i.e. daily or occasionally smokers, and one in seven were daily smokers in the Nordic region. The proportion of smokers was higher in men than in women ( $22 \%$ vs. $20 \%$ ) and in $18-24$ - and $25-44-y$-olds than in $45-65-y$ olds. No gender difference was found for daily smokers, but the proportion of daily smokers was higher in $45-65$-y-olds than in $18-24-y$-olds. The proportions of smokers and daily smokers decreased with increasing level of education. According to other national estimates from the Nordic countries the proportions of smokers and daily smokers have been declining in the Nordic countries during the last decade.

In 2014, the mean frequency of total alcohol consumption was 1.7 times/week and $45 \%$ were binge drinkers at least once during the last month among adults in the Nordic region. Mean total alcohol consumption and the proportion of binge drinkers were higher in men than in women ( 2.2 vs. 1.3 times/week and $56 \%$ vs. $34 \%$ ). Furthermore, mean total alcohol consumption was higher in 45-65-y-olds than in 18-24- and 25-44$y$-olds, and higher among the high educated than among the medium and low educated, whereas the proportion of binge drinkers decreased across age groups and was higher among the medium educated than among the high educated. According to other national estimates from the Nordic countries the proportions exceeding the weekly alcohol limits and binge drinkers did not change or decreased in the Nordic countries during the last decade.

\subsection{Children in the Nordic region}

In 2014, one in seven children had an unhealthy diet in the Nordic region. The proportion with an unhealthy diet was higher in boys than in girls (18\% vs. $14 \%$ ). The proportion with an unhealthy diet did not change between 2011 and 2014, neither in boys nor in girls (Table 28 and 30 in the chapter on diet). The proportion with an unhealthy diet was higher in children with a low or medium educated parent than in children with a 
high educated parent, and it increased in children with a low educated parent and decreased in children with a high educated parent (Table 32 in the chapter on diet).

In 2014, six in ten children were physically inactive in the Nordic region. The proportion of inactive was higher in girls than in boys (64\% vs. 54\%). The proportion of inactive did not change between 2011 and 2014 in the Nordic region (Figure 10). This was also found when analysing gender and parental education. In girls, the proportion of highly inactive decreased from 2011 to 2014.

In 2014, more than one in seven children spent more than four hours daily on recreational screen time in the Nordic region (Figure 10). The proportion with high recreational screen time was higher in boys than in girls (18\% vs. $13 \%)$. The proportion with high recreational screen time did not change between 2011 and 2014 among children in the Nordic region. No significant change was also found when parental education was analysed (Table 55 in the chapter on physical activity). However, the proportion with high recreational screen time decreased in girls from 2011 to 2014 while no change was found in boys.

In 2014, more than one in seven children were overweight or obese and $3 \%$ were obese in the Nordic region (Figure 10). The prevalence of OW/OB and OB did not differ between genders. The prevalence of OW/OB and OB did not change between 2011 and 2014 indicating that the OB prevalence among children in the Nordic countries is on a plateau. However, OW/OB prevalence increased among girls, indicating a risk of a further rise in both $O W / O B$ and $O B$ among children (Table 69 in the chapter on overweight and obesity). The prevalence of OW/OB increased between 2011 and 2014 in children with a high educated parent (Table 70 in the chapter on overweight and obesity). 
Figure 10: Population levels and the development in the proportions with an unhealthy diet, inactive and with high recreational screen time and the prevalence of $O W / O B$ and $O B$ among children (7-12 years) in the Nordic region

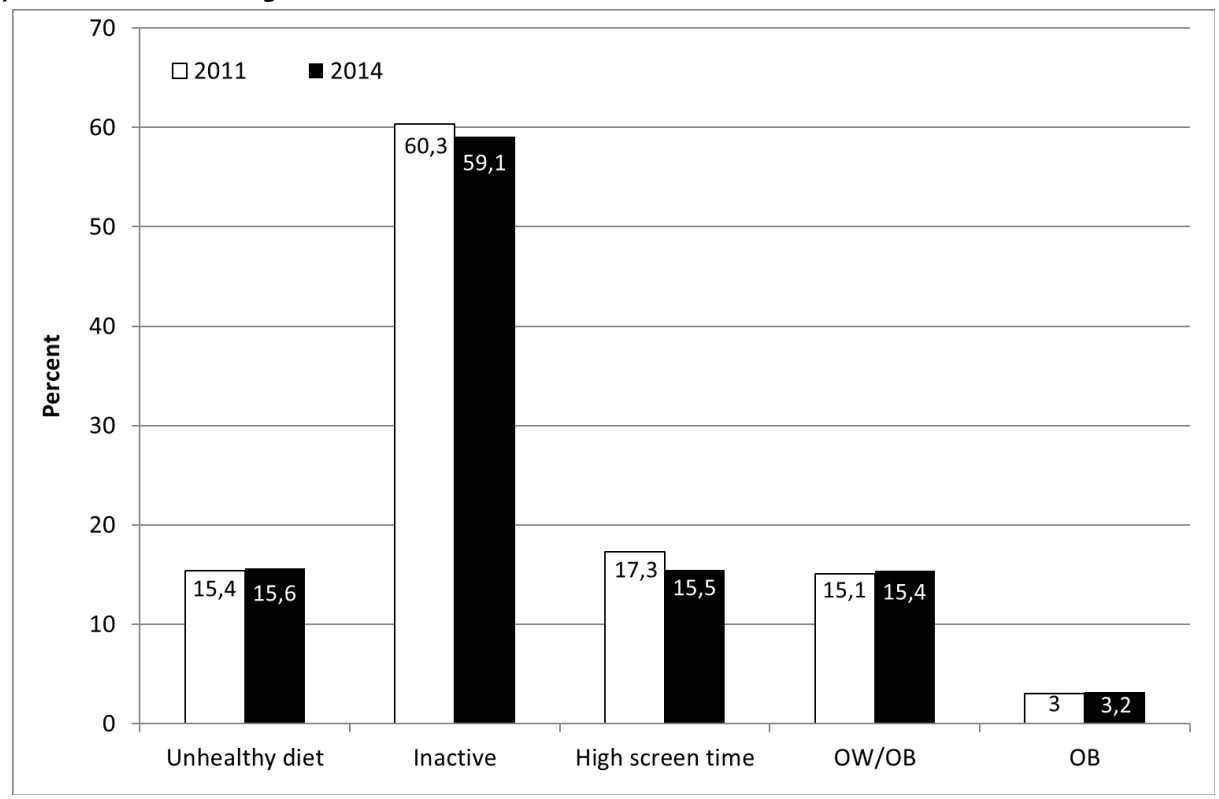

\subsection{Natural fluctuations or downward/upward temporal trends}

The increases in the prevalence of $\mathrm{OB}$ and in the proportions with an unhealthy diet, highly inactive and with high recreational screen time are all unfavorable developments among adults in the Nordic region. Even though the increases only are 1 to 3 percentage points, it is of great concern because the analysed time period is only three years. On the other hand, it cannot be ruled out that some of the significant changes in NORMO may be due to natural variation influenced by different factors such as participation rate. Thus, based on two data collections, three years apart, it is too early to make too firm interpretations of the situation and the development in the Nordic region. The Nordic monitoring has to be repeated before temporal trends may be interpreted more distinctly. 


\subsection{The most and least favorable health behaviour and weight status in the five Nordic countries}

In the following section, the most and least favorable health behaviour and weight status has been compared in the five Nordic countries among adults and children using data from NORMO 2014 (Table 90 and 91).

Table 90: Nordic countries with the most and least favourable health behaviour and weight status among adults according to data from NORMO 2014. A dash means that it is not possible to identify one or more countries as the most or least favourable

Most favorable

Least favorable

Diet

Unhealthy diet (\%)

Fruits and vegetables (freq./day)

Fish (freq./week)

Whole-grain bread (freq./day)

High intake of foods rich in saturated fat (\%)

High intake of sugar-rich foods (\%)

$\begin{array}{ll}\begin{array}{l}\text { Norway, Finland } \\ \text { Sweden }\end{array} & \begin{array}{l}\text { Sweden } \\ \text { Iceland } \\ \text { Iceland }\end{array} \\ \begin{array}{l}\text { Denmark } \\ \text { Norway } \\ \text { Denmark }\end{array} & \begin{array}{l}\text { Iceland } \\ \text { Sweden } \\ \text { Iceland }\end{array} \\ \text { Finland, Sweden } & \text { Norway } \\ \text { Sweden, Iceland } & - \\ \text { Sweden } & \\ \text { Sweden } & - \\ \text { Iceland } & \text { Denmark } \\ \text { Sweden, Iceland } & \\ & \text { Denmark } \\ \text { Denmark, Finland, Sweden, Norway } & \text { Norway } \\ \text { Sweden } & \text { Iceland } \\ \end{array}$

Physical activity and sedentary behaviour

Inactive (\%)

High recreational screen time (> 4h/day) (\%)

Smoking

Smokers (\%)

Daily smokers (\%)

Sweden

Iceland 
Table 91: Nordic countries with the most and least favourable health behaviour and weight status among children according to data from NORMO 2014. A dash means that it is not possible to identify one or more countries as the most or least favourable

\section{Most favorable L Least favorable}

Diet

Unhealthy diet (\%)

Fruits and vegetables (freq./day)

Fish (freq./week)

Whole-grain bread (freq./day)

High intake of foods rich in saturated fat (\%)

High intake of sugar-rich foods (\%)

Physical activity and sedentary behaviour

Inactive (\%)

High recreational screen time (> 4h/day) (\%)

Weight status

$\mathrm{OW} / \mathrm{OB}(\%)$

$\mathrm{OB}(\%)$

$\begin{array}{ll}\text { - } & \text { Sweden } \\ \text { Denmark, Sweden } & \text { Iceland, Norway } \\ \text { Iceland } & \text { Denmark } \\ \text { Norway } & \text { Sweden, Iceland } \\ \text { Denmark } & - \\ \text { Norway } & - \\ & \\ \text { Finland } & \text { Sweden } \\ \text { Iceland } & -\end{array}$

Denmark had the most favorable intake of foods rich in saturated fat among adults and children (lowest proportion with a high intake of foods rich in saturated fat) in the Nordic countries and the most favorable intake of fruits and vegetable among children. But Denmark also had the least favorable intake of fish among adults and children and the least favorable daily smoking and alcohol behaviour among adults.

Finland had the most favorable diet (lowest proportion with an unhealthy diet) among adults and the most favorable physical activity behaviour (lowest proportion of inactive) among adults and children.

Sweden had the lowest prevalence of $O B$ among adults in the Nordic countries and the most favorable health behaviour as regards intake of fruit and vegetables among adults and children and regarding physical activity, sedentary behaviour (lowest proportion with high recreational screen time), smoking and binge drinking among adults. However, Sweden also had the least favorable diet (highest proportion with an unhealthy diet) among adults and children and the highest proportion of inactive children. The intake of foods rich in saturated fat was not favorable among adults and the intake of whole grain bread was not favorable among children in Sweden compared to the other Nordic countries.

Iceland was the Nordic country with the most favorable intake of fish among adults and children, the most favorable sedentary behaviour among adults and children and the most favorable alcohol behaviour (lowest mean total alcohol consumption) among adults. But Iceland was also the country with the least favorable intake of fruits and vegetables and whole grain bread among adults and children and the least favorable 
sugar intake (highest proportion with a high intake of sugar-rich foods) among adults. In addition, Iceland had the biggest weight problem of the Nordic countries because of the high $O W / O B$ and $O B$ prevalence among adults.

Finally, Norway was the Nordic country with most favorable diet among adults and the most favorable whole grain bread and sugar intake (lowest proportion with a high intake of sugar-rich foods) among adults and children, but Norway also had the highest proportions of inactive and binge drinkers among adults and the least favorable intake of fruit and vegetables among children.

In summary, the main findings show that the magnitude of the challenges in the different areas differ between the five Nordic countries. However, there is still a need for improvements in all of the Nordic countries in many areas. All of the Nordic countries, except Finland are challenged with some specific unfavorable population levels in health behaviour and weight status among adults and/or children compared to the other Nordic countries.

\subsection{The development in the five Nordic countries and in the Nor- dic region}

Table 92 and 93 gives an overview on how the key variables on diet, physical activity and overweight developed from 2011 to 2014 among adults and children, respectively in each of the five Nordic countries and in the Nordic region. 
Table 92: The development in health behaviour and weight status among adults in the Nordic countries and in the Nordic region (favorable, unchanged, unfavorable). NORMO 2011 and 2014

DK

$\mathrm{FI}$

SE

NO

Nordic region@

Diet

\begin{tabular}{|c|c|c|c|c|c|c|}
\hline Unhealthy diet (\%) & Unchanged & Unchanged & Unfavorable & Unfavorable & Unfavorable & Unfavorable \\
\hline $\begin{array}{l}\text { Fruit \& vegetables } \\
\text { (freq./day) }\end{array}$ & Unchanged & Favorable & Favorable & Unchanged & Unfavorable & Unchanged \\
\hline Fish (freq./day) & Unfavorable & Unchanged & Unchanged & Unchanged & Unfavorable & Unfavorable \\
\hline $\begin{array}{l}\text { Whole-grain bread } \\
\text { (slices/day) }\end{array}$ & Unfavorable & Unfavorable & Unfavorable & Unfavorable & Unfavorable & Unfavorable \\
\hline $\begin{array}{l}\text { High intake of foods rich in } \\
\text { saturated fat }(\%)\end{array}$ & Unchanged & Unchanged & Unfavorable & Unfavorable & Unfavorable & Unfavorable \\
\hline $\begin{array}{l}\text { High intake of sugar-rich } \\
\text { foods }(\%)\end{array}$ & Favorable & Favorable & Unchanged & Unchanged & Favorable & Favorable \\
\hline \multicolumn{7}{|l|}{$\begin{array}{l}\text { Physical activity and seden- } \\
\text { tary behaviour }\end{array}$} \\
\hline Inactive (\%) & Unchanged & Unchanged & Unchanged & Unchanged & Unchanged & Unchanged \\
\hline $\begin{array}{l}\text { High recreational screen } \\
\text { time (> } 4 \mathrm{~h} / \text { day })(\%)\end{array}$ & Unfavorable & Unfavorable & Unchanged & Unfavorable & Favorable & Unfavorable \\
\hline \multicolumn{7}{|l|}{ Weight status } \\
\hline OW/OB (\%) & Unchanged & Unchanged & Unchanged & Unchanged & Unchanged & Unchanged \\
\hline $\mathrm{OB}(\%)$ & Unchanged & Unchanged & Unchanged & Unfavorable & Unfavorable & Unfavorable \\
\hline
\end{tabular}

Note: @ Weighted according to population size in the five Nordic countries.

\subsubsection{Adults}

Among adults in the Nordic region, the overall diet quality (unhealthy diet), sedentary behaviour (high recreational screen time) and the prevalence of OB developed unfavorably from 2011 to 2014 while no change was seen in physical activity (inactive) and in the prevalence of OW/OB. The unfavorable development in the key variables of health behaviour and OB prevalence was evident in especially Iceland, but also in Norway. Nevertheless, an unfavorable development was also seen in Denmark and Finland with regard to sedentary behaviour and in Sweden with regard to the proportion with an unhealthy diet.

The only favorable development among adults in the Nordic region was the decrease in the proportion with a high consumption of sugar-rich foods. This was also seen in Denmark, Finland and Norway while no change was found in Sweden and Iceland. In 
the Nordic region, the proportion with an unhealthy diet increased and this unfavorable development was also seen for the intake of fish and whole grain bread (decreases) and the proportion with a high intake of foods rich in saturated fat (increase).

The decrease in the intake of whole grain bread was seen in all the Nordic countries, while the decrease in the intake of fish was seen in Denmark and Norway; and the increase in the proportion with a high intake of foods rich in saturated fat was seen in Sweden, Iceland and Norway.

There was no change in the intake of fruit and vegetables in the Nordic region, but an increase was found in Finland and Sweden and a decrease in Norway. When looking at the proportion meeting " $5+$ a day", an increase was also observed in Finland while a decrease was seen in Norway (Table 15 in the chapter on diet).

The proportions eating fish as a main meal twice a week and eating at least half of their daily bread intake as whole grain bread also decreased slightly in the Nordic region. The proportion eating fish as a main meal twice a week decreased in Denmark and Norway and the proportion eating at least half of their daily bread intake as whole grain bread decreased slightly in Finland and Iceland (Table 15 in the chapter on diet).

The unfavorable development in the proportion with high recreational screen time (increased) was seen in the Nordic region and in Denmark, Finland and Iceland while no change was seen in Sweden and a decrease was found in Norway. Differences due to gender, age, and education are described in the chapters of the report.

The development in OW/OB did not change among adults in the Nordic region as well as in the Nordic countries. The unfavorable OB development among adults in the Nordic region was also seen in Iceland and Norway while no significant change was observed in Denmark, Finland and Sweden.

\subsubsection{Children}

Among children in the Nordic region, there was no overall change in diet (unhealthy diet), physical activity (inactive), sedentary behaviour (high recreational screen time) and OW/OB and OB prevalence between 2011 and 2014. This was also seen in each of the five Nordic countries, except in Finland with a decrease in the proportion with an unhealthy diet and in Norway with a decrease in the proportion of inactive.

Although the proportion with an unhealthy diet did not change in the Nordic region, changes were found when analysing the diet in more details. The favorable changes were a decrease in the proportion with a high consumption of sugar-rich foods, and increased intake of fruits and vegetables and fish, including fish as a main meal twice a week, while the proportion eating " $5+$ a day" did not change (Table 26 and 27 in the chapter on diet). The favorable development in the intake of fruits and vegetables 
was seen in Finland and Sweden. and the favorable development in the intake of fish was seen in Denmark, Finland and Iceland.

The development in the intake of whole grain bread was however not favorable among children in the Nordic region, and this was also seen in Iceland and Norway. However, while the proportion eating at least half of their daily bread intake as whole grain bread did not change in the Nordic region, the development in the proportion eating half of their daily bread intake as whole grain was only unfavorable in Norway (Table 26 in the chapter on diet).

Table 93: The development in health behaviour and weight status among children in the Nordic countries and in the Nordic region (favorable, unchanged, unfavorable). NORMO 2011 and 2014

\begin{tabular}{|c|c|c|c|c|c|c|}
\hline & DK & FI & SE & IS & NO & Nordic region@ \\
\hline \multicolumn{7}{|l|}{ Diet } \\
\hline Unhealthy diet (\%) & Unchanged & Favorable & Unchanged & Unchanged & Unchanged & Unchanged \\
\hline $\begin{array}{l}\text { Fruits and vegetables } \\
\text { (freq./day) }\end{array}$ & Unchanged & Favorable & Favorable & Unchanged & Unchanged & Favorable \\
\hline Fish (freq./week) & Favorable & Favorable & Unchanged & Favorable & Unchanged & Favorable \\
\hline $\begin{array}{l}\text { Whole-grain bread } \\
\text { (slices/day) }\end{array}$ & Unchanged & Unchanged & Unchanged & Unfavorable & Unfavorable & Unfavorable \\
\hline $\begin{array}{l}\text { High intake of foods rich } \\
\text { in saturated fat }(\%)\end{array}$ & Favorable & Unchanged & Unchanged & Unchanged & Unchanged & Unchanged \\
\hline $\begin{array}{l}\text { High intake of sugar-rich } \\
\text { foods }(\%)\end{array}$ & Unchanged & Unchanged & Unchanged & Unchanged & Unchanged & Favorable \\
\hline \multicolumn{7}{|l|}{$\begin{array}{l}\text { Physical activity and sed- } \\
\text { entary behaviour }\end{array}$} \\
\hline Inactive (\%) & Unchanged & Unchanged & Unchanged & Unchanged & Favorable & Unchanged \\
\hline $\begin{array}{l}\text { High recreational screen } \\
\text { time }(>4 \text { h/day) }(\%)\end{array}$ & Unchanged & Unchanged & Unchanged & Unchanged & Unchanged & Unchanged \\
\hline \multicolumn{7}{|l|}{ Weight status } \\
\hline OW/OB (\%) & Unchanged & Unchanged & Unchanged & Unchanged & Unchanged & Unchanged \\
\hline $\mathrm{OB}(\%)$ & Unchanged & Unchanged & Unchanged & Unchanged & Unchanged & Unchanged \\
\hline
\end{tabular}

Note: @Weighted according to population size in the five Nordic countries.

\subsubsection{Comparison with other national estimates in the Nordic countries}

Other national estimates from the Nordic countries are to some extent in line with NORMO data when the development of diet, physical activity and overweight are compared. However, other national estimates on the dietary intake are few and the discrep- 
ancies between other national estimates and NORMO data are probably due to different participation rates, age groups, survey years, dietary assessment methods and definitions used.

\subsubsection{Summary of the development and the challenges in the Nordic region and the Nordic countries}

Results indicate that the development of diet, physical activity and overweight is heading in the wrong direction among adults in the Nordic region, while the development among children is more favorable. There is still a need for improvements in all the Nordic countries in all areas. Each of the Nordic countries is challenged with some specific unfavorable population levels and/or development in health behaviour and weight status among adults and/or children.

Denmark is challenged with unfavorable smoking and alcohol behaviour among adults. The high population levels of daily smoking and total alcohol consumption are a major threat to public health due to the increased risk of premature death and chronic diseases.

Sedentary behaviour may be an increasing public health challenge among adults in Finland taking the most recent development of high recreational screen time into consideration.

The population level and the development of the proportion with an unhealthy diet in Sweden are of concern, especially among adults. In addition, the high proportion of inactive children in Sweden is worrying from a public health point perspective even if the level did not change between 2011 and 2014. Sweden is far away from fulfilling the common Nordic ambition about all children being physically active in 2021.

The current situation and the development of OW/OB and OB indicate that this is a major public health problem among adults in Iceland.

Finally, the high proportion of inactive among adults in Norway may give rise to concern even if no change was detected between 2011 and 2014.

\subsubsection{Interrelationship between results on diet, physical activity and overweight}

Changes in obesity-promoting factors such as unhealthy diet, physical inactivity and sedentary behaviour might have contributed to the observed $\mathrm{OB}$ increase between 2011 and 2014 among adults in the Nordic region. There have been increases among adults eating an unhealthy diet, and in the proportions of highly inactive and sedentary 
individuals in the Nordic countries. These findings may be linked to the observed OB increase among adults in the Nordic countries.

Results show that the prevalence of $\mathrm{OB}$ increased in women, but not in men. The proportion with an unhealthy diet increased in both men and women between 2011 and 2014, but the increase was higher in men than in women. The development of physical activity was however different between genders because the proportion of highly inactive increased in women whereas the proportion of highly active increased in men. Even if these findings may shed some light on the OB increase found in women, they should be interpreted with caution because results on diet and physical activity did not strongly support the different development in $\mathrm{OB}$ among men and women.

In 2014, the highest prevalence of OB among adults was found in Iceland and the lowest in Sweden. When comparing Iceland and Sweden, a slightly higher proportion with an unhealthy diet was found in Sweden than in Iceland although the consumption of sugar-rich foods was higher and the consumption of fruits and vegetables was lower in Iceland compared with Sweden. The observed differences in OB may also be explained by physical activity and sedentary behaviour. The amount of moderate and vigorous intensity physical activity was lower in Iceland than in Sweden while recreational screen time was higher in Iceland. These differences were mirrored in higher proportions of inactive and highly inactive in Iceland compared with Sweden. Overall, these results indicate that the lifestyle among adults in Iceland is less active and more sedentary compared with Sweden. This may be part of the explanation of the large differences in OB prevalence between the two countries.

There was no change in the prevalence of OW/OB between 2011 and 2014 among children in the Nordic region, which is in line with the unchanged proportion with an unhealthy diet and the unchanged level of physical activity and sedentary behaviour.

\subsubsection{Evaluation of Goals 2011 and Visions 2021 for diet, physical activity and overweight}

The common Nordic ambitions of better health through diet and physical activity still have a long way to go among adults and children when evaluating Goals 2011 and Visions 2021 for diet, physical activity and overweight in the Nordic Plan of Action (see Table 94 and 95). 
Adults

Four in ten of the goals and one in ten of the visions in the Nordic Plan of Action seem to have been fulfilled among adults in the Nordic countries.

One goal that has been successfully fulfilled is the goal with regard to the intake of added sugar which decreased. In addition, the goals with regard to social inequality in diet, physical activity and overweight also seem to be fulfilled as social inequality in diet, physical activity and overweight did not change or decreased slightly.

However, the way goals on social inequality have been fulfilled was not always beneficial from a public health perspective. Social inequality in OB decreased through an increase in the prevalence of $O B$ in the high education group. This is not encouraging when the ambitions are to achieve better health in all social groups.

The only vision that seems to be currently fulfilled is the one regarding whole grain bread intake among adults in the Nordic region. $95 \%$ has a daily intake of whole grain bread corresponding to at least half of their daily intake of bread and the target level is $\geq 70 \%$. However, this vision needs a critical revision. The proportion eating at least half of their daily bread intake as whole grain bread is not necessarily a good indicator of the total intake of whole grain. Firstly, other sources of whole grain might be as important as bread in some countries, but not covered with NORMO data, and secondly, the whole grain bread variable shows quite high values even though the intake is low and decreasing, and the higher values can occur due to a lower intake of white bread. Therefore these results must be interpreted with caution.

Some of the visions may be possible to fulfill in 2021 such as the visions on the intake of added sugar, the target population level of physical activity and social inequality in physical activity and overweight, while other visions such as the intake of fruits and vegetables, fish, and saturated fat, the target prevalence of overweight and obesity and social inequality in diet will be difficult to reach in 2021.

\section{Children}

The common Nordic ambitions of better health through diet and physical activity are better off for children than adults, but children also have a long way to go to get a healthier lifestyle and weight status (see Table 95). Seven in ten of the goals and three in ten of the visions in the Nordic Plan of Action seem to have been fulfilled among children in the Nordic region. Goals that have been successfully fulfilled are an increase in the intake of fish, and fruits and vegetables, and a decrease in the intake of added sugar. In addition, the level of physical activity and sedentary behaviour and the prevalence of OW/OB and OB did not change, why Goal 2011 has been fulfilled. 
Finally, social inequality in physical activity and overweight developed in favorably among children in Nordic region. See however, the reservations made above in the adult section.

The visions that are currently fulfilled are the high successful intake of whole grain bread and the target difference of social inequality in physical activity and overweight. However, the same reservations have to be made to these conclusions since the visions need critical revision. The vision that may be possible to fulfill is the target prevalence of $\mathrm{OB}$, while the visions on the intake of fruits and vegetables, fish, saturated fat and added sugar, target population level of physical activity, prevalence of OW/OB and social inequality in diet will be difficult to reach in 2021.

Table 94: Evaluation of Goals 2011 and Visions 2021 for diet, physical activity and overweight in the Nordic Plan of Action among adults in the Nordic region. The goals and visions are shown in Appendix $C$

\section{Goal 2011}

Not fulfilled because the intake of fruits and vegetables did not change (mean freq./day: from 2.6 in 2011 to 2.5 in 2014).

Not fulfilled because the intake of fish decreased slightly (mean freq./week: from 1.5 in 2011 to 1.5 in 2014). ${ }^{10}$

Not fulfilled because the intake of whole grain bread/cereals decreased (mean slices/day: from 3.7 in 2011 to 3.3 in 2014). Needs revision. ${ }^{11}$

Not fulfilled because the intake of saturated fat increased (proportion with a high intake of foods rich in saturated fat: from $39 \%$ in 2011 to $42 \%$ in 2014).

Fulfilled because the intake of added sugar decreased (proportion with a high intake of sugarrich foods: from $30 \%$ in 2011 to $27 \%$ in 2014).

\section{Vision 2021}

Currently not fulfilled because $13 \%$ has an intake of fruits and vegetables of at least $500 \mathrm{~g} /$ day and the target population level is $\geq 70 \%$.

Currently not fulfilled because $37 \%$ consume fish twice a week and the target population level is $\geq 70 \%$.

Currently fulfilled because $95 \%$ has a daily intake of whole grain bread/cereals corresponding to at least half of their daily intake of bread/cereals and the target population level is $\geq 70 \%$. Needs revision. ${ }^{13}$

Currently not fulfilled according to other national estimates from the Nordic countries. Even if $64-83 \%{ }^{12}$ meet the NNR recommendation on fat (25-40 E\% from fat) and the target population level is $\geq 70 \%$, Vision 2021 is not fulfilled because the mean saturated fat intake of $13-15 \mathrm{E} \%$ does not meet the NNR recommendations (<10 E\%). ${ }^{13}$

Currently not fulfilled according to other national estimates from the Nordic countries because $63-76 \%$ meet the NNR recommendation on added sugar and the target population level is $\geq 80 \% .^{14}$

\footnotetext{
${ }^{10} \mathrm{~A}$ significant, but very small decrease corresponding to 0.07 times less intake of fish per week was found.

${ }^{11}$ Assessment of whole grain bread/cereals needs a critical revision since the variable shows quite high values even though the intake of whole grain bread is low and decreasing, and depending on the intake of white bread.

${ }^{12}$ Estimates from Proportion with a diet of total fat 25-40 E\% (\%) in 2010-2013 in Table 34

${ }^{13}$ Estimates from Saturated fat (E\%) in 2010-2013 in Table 34.

${ }^{14}$ Estimates from Proportion with a diet < 10 E\% added sugar (\%) in 2010-2013 in Table 34.
} 
Table 94 Continued

Physical Activity

Not fulfilled. Even if the proportion of inactive did not change (from $33 \%$ in 2011 to $34 \%$ in 2014), Goal 2011 is not fulfilled because the proportions of highly inactive (from $11 \%$ in 2011 to $12 \%$ in 2014) and with high recreational screen time (from $29 \%$ in 2011 to $30 \%$ in 2014) increased.

Overweight and Obesity

Not fulfilled. Even if the prevalence of $O W / O B$ did not change ( $47 \%$ in 2011 and 2014), Goal 2011 is not fulfilled because the prevalence of OB increased (from $11 \%$ in 2011 to $13 \%$ in 2014).

Social inequality in diet

Fulfilled because the social difference in diet did not change (social difference: from $54 \%$ in 2011 to $51 \%$ in 2014). ${ }^{15}$ Needs revision. ${ }^{16}$

Social inequality in physical activ-

ity

Social inequality in overweight and obesity
Fulfilled because the social difference in physical activity did not change (social difference: from $26 \%$ in 2011 to $22 \%$ in 2014). Needs revision. ${ }^{18}$

Fulfilled because the social difference in $O W / O B$ (social difference: from $38 \%$ to $31 \%$ ) and $O B$ (social difference: from $93 \%$ to $61 \%$ ) decreased. Needs revision. ${ }^{18}$
Currently not fulfilled because $66 \%$ is physically active (meets the physical activity recommendations) and the target population level is $\geq 75 \%$.

Currently not fulfilled because the prevalence of $O W / O B$ and $O B$ is $47 \%$ and $13 \%$, respectively, and the target prevalence is $34 \%$ and $9 \%$, respectively.

Currently not fulfilled. Vision 2021 is not fulfilled because the social difference in diet did not change and is $51 \%$ and the target difference is $\leq 20 \%$. Needs revision. ${ }^{18}$

Currently not fulfilled. Vision 2021 is not fulfilled because the social difference in physical activity did not change and is $22 \%$. and the target difference is $\leq 20 \%$. Needs revision. ${ }^{18}$

Currently not fulfilled. Even if the social difference in $O W / O B$ decreased, Vision 2021 is not fulfilled because the social difference in $O W / O B$ is $31 \%$ and the target difference is $\leq 20 \%$. Needs revision. ${ }^{18}$

Table 95: Evaluation of Goals 2011 and Visions 2021 for diet, physical activity and overweight in the Nordic Plan of Action among children in the Nordic region. The goals and visions are shown in Appendix $C$

\begin{tabular}{|c|c|c|}
\hline & Goal 2011 & Vision 2021 \\
\hline \multirow[t]{3}{*}{ Diet } & $\begin{array}{l}\text { Fulfilled because the intake of fruits and vegetables } \\
\text { increased slightly (mean freq./day: from } 2.8 \text { in } 2011 \\
\text { to } 2.9 \text { in 2014). }\end{array}$ & $\begin{array}{l}\text { Currently not fulfilled because } 15 \% \text { has an intake of fruits and vege- } \\
\text { tables of at least } 500 \mathrm{~g} / \text { day and the target population level is } \geq \\
70 \% \text {. }\end{array}$ \\
\hline & $\begin{array}{l}\text { Fulfilled because the intake of fish increased slightly } \\
\text { (mean freq./week: from } 1.3 \text { in } 2011 \text { to } 1.4 \text { in 2014). }\end{array}$ & $\begin{array}{l}\text { Currently not fulfilled because } 42 \% \text { consume fish twice a week } \\
\text { and the target population level is } \geq 70 \% \text {. }\end{array}$ \\
\hline & $\begin{array}{l}\text { Not fulfilled because the intake of whole grain bread/ce- } \\
\text { reals decreased (mean slices/day: from } 3.3 \text { in } 2011 \text { to } \\
3.1 \text { in 2014). Needs revision. }{ }^{17}\end{array}$ & $\begin{array}{l}\text { Currently fulfilled because } 88 \% \text { has a daily intake of whole grain } \\
\text { bread/cereals corresponding to at least half of their daily intake } \\
\text { of bread/cereals and the target population level is } \geq 70 \% \text { Needs } \\
\text { revision. }{ }^{19}\end{array}$ \\
\hline
\end{tabular}

${ }^{15}$ Relative difference in diet (unhealthy diet), physical activity (inactive), OW/OB and OB between the low and high education group ${ }^{16}$ Goals and visions on social inequality need a critical revision because the way these have been fulfilled was not always beneficial from a public health perspective. Fulfillment of the goals and visions occurred sometimes through deteriorated health behaviour and health status in the high education group.

${ }^{17}$ Assessment of whole grain bread/cereals needs a critical revision since the variable shows quite high values even though the intake of whole grain bread is low and decreasing, and depending on the intake of white bread. 


\section{Goal 2011}

Vision 2021

Table 95 Continued

Not fulfilled because the intake of saturated fat did not change (proportion with a high intake of foods rich in saturated fat: 34\% in 2011 and 2014).

Fulfilled because the intake of added sugar decreased (proportion with a high intake of sugar-rich foods: from $21 \%$ in 2011 to $19 \%$ in 2014).

Physical Activity

Fulfilled because the proportions of inactive (from $60 \%$ in 2011 to $59 \%$ in 2014) and with high recreational screen time ( $17 \%$ vs. $16 \%$ ) did not change.

Overweight and Obesity

Social inequality in diet

Social inequality in physical activity

Social inequality in overweight and obesity
Fulfilled because the prevalence of $O W / O B(15 \%$ in 2011 and 2014) and $O B$ (3.0\% in 2011 and 2014) did not change.

Not fulfilled because the social difference in diet increased considerably (social difference: from $-10 \%$ in 2011 to $133 \%$ in 2014). ${ }^{21}$ Needs revision. ${ }^{18}$

Fulfilled because the social difference in physical activity did not change (social difference: from $1 \%$ in 2011 to $-5 \%$ in 2014). Needs revision. ${ }^{18}$

Fulfilled because the social difference in $O W / O B$ (social difference: from $98 \%$ to $19 \%$ ) and $O B$ (social difference: from $345 \%$ to $148 \%$ ) decreased considerably. Needs revision. ${ }^{18}$
Currently not fulfilled according to other national estimates from the Nordic countries. Even if $85-99 \%{ }^{18}$ meet the NNR recommendation on fat ( $25-40 \mathrm{E} \%$ from fat) and the target population level is $\geq 70 \%$, Vision 2021 is not fulfilled because the mean saturated fat intake of 8-14 E\% does not meet the NNR recommendations ( $<10$ $\mathrm{E} \%)^{19}$

Currently not fulfilled according to other national estimates from the other Nordic countries because $13-67 \%$ meet the NNR recommendation on added sugar and the target population level is $\geq$ $80 \% .{ }^{20}$

Currently not fulfilled because $41 \%$ is physically active (meets the physical activity recommendations) and the target population level is $100 \%$.

Currently not fulfilled because the prevalence of $O W / O B$ and $O B$ is $15 \%$ and $3 \%$, respectively, and the target prevalence is $9 \%$ and $2 \%$, respectively.

Currently not fulfilled because the social difference in diet increased considerably and is $133 \%$ and the target difference is $\leq$ $20 \%$. Needs revision. ${ }^{18}$

Currently fulfilled. Vision 2021 is fulfilled because the social difference in physical activity is $5 \%$ and the target difference is $\leq$ $20 \%$. Needs revision ${ }^{18}$

Currently fulfilled because the social difference in $O W / O B$ decreased considerably and is $19 \%$ and the target difference is $\leq$ $20 \%$. Needs revision. ${ }^{18}$

\footnotetext{
${ }^{18}$ Estimates from Proportion with a diet of total fat 25-40 E\% (\%) in 2011-2014 in Table 35.

${ }^{19}$ Estimates from Saturated fat (E\%) in 2011-2014 in Table 35. Only children in Norway have a mean saturated fat intake below the recommended level.

${ }^{20}$ Estimates from Proportion with a diet < 10 E\% added sugar (\%) in 2011-2014 in Table 35.

${ }^{21}$ Relative difference in diet (unhealthy diet), physical activity (inactive), OW/OB and OB between the low and high education group
} 


\section{Conclusion}

The Nordic Monitoring System comprises data collected in autumn 2011 and 2014. Results from 2014 represent the most recent population levels on diet, physical activity, smoking, alcohol and overweight in the Nordic countries. The emphasis in presenting the results has been put on the key findings in the Nordic region, including in relation to gender, age groups and education level. Furthermore, emphasis has been put on showing the development of the key variables, but the population levels in 2014 and selected data on country level is also described.

The overall picture shows that one in five adults had an unhealthy diet in the Nordic region, while only one in 10 adults had a healthy diet in 2014. One in three adults were physically inactive and three in ten adults spent more than four hours daily on recreational screen time. Furthermore, one in five adults were smokers (daily or occasionally smokers) and one in seven were daily smokers in the Nordic region. Mean total alcohol consumption among adults was 1.7 times/week and the proportion of binge drinkers was $45 \%$. Finally, the prevalence of OW/OB and OB among adults was high in the Nordic region. Today, it is almost as common to be overweight or obese as an adult as being normal weight.

The increase from 2011 to 2014 in OB prevalence and in the proportions with an unhealthy diet, of highly inactive and with high recreational screen time among adults in the Nordic region is of concern. Even so, an encouraging increase in the proportion of highly active was also found. These findings could indicate that an increased polarisation of the physical activity pattern is evident among adults in the Nordic region.

Results show that health behaviour and weight status, i.e. diet, sedentary behaviour, smoking, alcohol, OW/OB and OB, were less favorable among men than among women in the Nordic region.

There were no changes in diet, physical activity and overweight between 2011 and 2014 among children in the Nordic region. Although it is encouraging that the same unfavorable development was not seen for children as for adults, the high population levels in 2014 are of concern. Even though the proportion of children with an unhealthy diet was $15 \%$, the proportion with a healthy diet was only $10 \%$. The proportion of inactive children was approx. $60 \%$ and therefore very high. Also the prevalence of OW/OB and $\mathrm{OB}$ was relative high; and a halt in the development has previously been followed by a rise. 
Gender differences in health behaviour and weight status are not as clear in children as in adults in the Nordic region. Diet and sedentary behaviour were less favorable among boys than among girls, whereas physical activity was less favorable among girls. Each of the five Nordic countries is challenged with some specific unfavorable population levels and/or development in health behaviour and/or weight status among adults and/or children compared to the other Nordic countries. However, there is still a need for improvements in all the Nordic countries in all areas, and the development among adults has mostly been unfavorable or unchanged, while the development has mostly been unchanged or favorable among children.

This is reflected in the evaluation of the results in relation to the Goals 2011 and Visions 2021 of the Nordic Plan of Action. The Goals 2011 aim at a favorable development in diet, physical activity and overweight, while the Visions 2021 aim at reaching a certain population level in 2021.

The goal of added sugar in the diet, "The intake of added sugar has been reduced", has been fulfilled among adults and children. Among adults this was mainly due to a reduction in the intake of cakes, while among children the reduction was mainly in candy and chocolate (Table 94 and 95 in the previous chapter and Table 96 and 97 on Appendix D).

In children, the four other goals that have been successfully fulfilled are: "The consumption of fruits and vegetables has increased", "The consumption of fish has increased", "The current trend, where an increasing proportion of children are physically inactive, has been brought to a halt and at best reversed", and "The continuing increase in the proportion of the overweight and obese children has stopped and at best reversed".

In addition, the goals with regard to social inequality in diet, physical activity and OW/OB also seem to be fulfilled since a decrease or no change in social inequality in diet, physical activity and overweight was found in both adults and children, except for children regarding diet. However, the way goals on social inequality have been fulfilled was not always beneficial from a public health perspective.

The only vision that seems to be currently fulfilled is the one regarding whole grain bread intake in both adults and children in the Nordic region. However, this vision needs a critical revision since the variable shows quite high values even though the intake of whole grain bread is low and decreasing. Further it is depending on the intake of white bread.

Some of the visions may be possible to fulfill in 2021 among adults in the Nordic region such as the visions on the intake of added sugar, the target population level of physical activity and social inequality in physical activity and overweight, while other visions such as the intake of fruits and vegetables, fish, and saturated fat, the target 
population level of $O W / O B$ and $O B$ and social inequality in diet will be difficult to reach in 2021.

The same picture is seen among children in the Nordic region. However, the vision that may be possible to fulfill is the target prevalence of $O B$, while the visions on added sugar and the target population level of physical activity will be difficult to reach in 2021.

The Nordic Monitoring System has now collected data for 2011 and 2014. In this short three year period, significant changes have occurred in the Nordic region, albeit some are modest in magnitude. Some of the significant changes in the results may be due to natural variation influenced by, among other factors, participation rate. Thus, based on two data collections, three years apart, it is too early to make too firm interpretations of the situation and the development in the Nordic region. The Nordic monitoring has to be repeated before temporal trends may be interpreted more distinctly.

The strength of the Nordic Monitoring System is the high comparability between survey years, age groups, educational levels and countries. Other national surveys cannot provide such comparability. However before a third data collection is started, initiatives to improve response rate especially in some of the Nordic countries are needed.

In summary, the big picture of the changes from 2011 to 2014 in diet, physical activity and overweight shows that the development is heading in the wrong direction among adults in the Nordic region while the development among children is more positive, but improvements are still needed. A coming third data collection would show if these tendicies will continue or if public health initiatives that target diet, physical activity and overweight among adults and children in the Nordic region will succeed in introducing improvements that leads to a healthier lifestyle and weight status. Men, $45-65-y$-olds and the low educated may be especially relevant groups to target to fulfill the common Nordic ambition of ensuring better health and quality of life on equal terms for all Nordic citizens. 



\section{References}

Amcoff E, Edberg A, Enghardt Barbieri H, Lindroos A-K, Nälsén C, Pearson M, Warensjö Lemming E (2012). Riksmaten - vuxna 2010-11. Livsmedels- och näringsintag bland vuxna i Sverige (in Swedish with summary in English: Riksmaten - adults 2010-2011: dietary habits and nutrient intake in Swedish adults).

Anderssen SA, Kolle E, Steene-Johannessen J, Ommundsen Y, Andersen LB (2008). Rapport: Fysisk aktivitet blant barn og unge i Norge. En kartlegging av aktivitetsnivå og fysisk form hos 9- og 15-åringer. Helsedirektoratet, Norway.

Atrostic BK, Bates N, Burt G, Silberstein A. Nonresponse in U.S. government household surveys: consistent measures, recent trends, and new insights. Journal of Official Statistics. 2001;17(2):209-226.

Becker W (2009). Indikatorer för bra matvanor. Resultat från intervjuundersökninger 2008. SLV rapport 22-2009. National Food Administration, Sweden.

Borodulin K (personal communication; 2015). Calculations based on data from the National FINRISK 2007 and 2012 Studies (2007 and 2012).

Borodulin K, Zimmer C, Sippola R, Makinen TE, Laatikainen T, Prattala R. Health behaviours as mediating pathways between socioeconomic position and body mass index. Int J Behav Med. 2012;19(1):14-22.

Borodulin K, Levälahti E, Saarikoski L, Lund L, Juolevi A, Grönholm M, Jula A, Laatikainen T, Männistö S, Peltonen M, Salomaa V, Sundvall J, Taimi M, Virtanen S, Vartiainen E. Kansallinen FINRISKI 2012 --terveystutkimus - Osa 2: Tutkimuksen taulukkoliite (2013). The National FINRISK Study 2012 - Tables). Terveyden ja hyvinvoinnin laitos, Raportti 2013/22.

Bye EK (personal communication; 2015). The Norwegian Institute for Alcohol and Drug Research (SIRUS).

Christensen Al, Ekholm O, Davidsen M, Juel K (2012). Danish Health and Morbidity Survey 2010 \& trends since 1987. Report, Statens Institut for Folkesundhed, Syddansk Universitet, København.

Chun YI, Robertson KW. The effects of advance letters and reminder/thank you letter on reducing nonrespone in an establishment survey: an experimental study. ASA Proceedings of the Section on Survey Research Methods. 1995:1,151-1,156.

Cohen G, Duffy JC. Are nonrespondents to health surveys less healthy than respondents? Journal of Official Statistics. 2002;18(1):13-23.

Cole TJ, Bellizzi MC, Flegal KM, Dietz WH (2000). Establishing a standard definition for child overweight and obesity worldwide: international survey. BMJ; 320: 1, 240-43.

Connor Gorber S, Tremblay M, Moher D, Gorber B. comparison of direct vs. self-report measures for assessing height, weight and body mass index: a systematic review. Obes Rev 2007;8:307-26.

Currie C, Gabhainn SN, Godeau E, Roberts C, Smith R, Currie D, Picket W, Richter M, Morgan A, Barnekow V, eds (2008). Inequalities in young people's health: Health Behaviour in School- 
aged Children (HBSC) study: international report from the 2005/2006 survey. Copenhagen, WHO Regional Office for Europe (Health Policy for Children and Adolescents, NO. 5).

Currie C, Zanotti C, Morgan A, Currie D, de Looze M, Roberts C, Samdal O, Smith ORF, Barnekow $V$, eds (2012). Social determinants of health and well-being among young people. Health Behaviour in School-aged Children (HBSC) study: international report from the 2009/2010 survey. Copenhagen, WHO Regional Office for Europe (Health Policy for Children and Adolescents, NO. 6).

Danish Health and Medicines Authority (2014). Health in Denmark. The Danish National Healthprofile 2013, Report.

de Heer W, Israels AZ. Response rates in Europe. ASA Proceedings of the Section on Survey Research Methods. 1992:92-101.

de Heer W. International response trends: results of an international survey. Journal of Official Statistics. 1999;15(2):129-142.

Demarest S, Van der Heyden J, Charafeddine R, Tafforeau J, Van Oyen H, Van Hal G. Socio-economic differences in participation of households in a Belgian national health survey. Eur $J$ Public Health. 23(6):981-985.

Doody MM, Sigurdson AS, Kampa D, Chimes K, Alexander BH, Ron E, Tarone RE, Linet M. Randomized trial of financial incentives and delivery methods for improving response to a mailed questionnaire. Am J Epidemiol. 2003;157(7):643-651.

Drivsholm T, Eplov LF, Davidsen M, Jørgensen T, Ibsen $H$, Hollnagel H, Borch-Johnsen K.. Representativeness in population-based studies: a detailed description of non-response in a Danish cohort study. Scand J Public Health. 2006;34(6):623-631.

Eaker S, Bergstrom R, Bergstrom A, Adami HO, Nyren O. Response rate to mailed epidemiologic questionnaires: a population-based randomized trial of variations in design and mailing routines. Am J Epidemiol. 1998;147(1):74-82.

Edwards P, Roberts I, Clarke M, Diguiseppi C, Pratap S, Wentz R, Kwan I. Increasing response rates to postal questionnaires: systematic review. BMJ. 2002;324(7,347):1,183.

Edwards PJ, Roberts I, Clarke MJ, Diguiseppi C, Wentz R, Kwan I, Cooper R, Felix LM, Pratap S. Methods to increase response to postal and electronic questionnaires (Review). The Cochrane Library. 2009 (Issue 1).

Enghardt Barbieri H, Pearson M, Becker W (2006). Riksmaten - barn 2003: livsmedels- och näringsintag bland barn i Sverige (In Swedish with summary in English: Riksmaten - children 2003: dietary habits and nutrient intake in Swedish children). National Food Administration, Sweden.

European Health Interview \& Health Examination Surveys Database. https://hishes.wivisp. be/index.php?hishes=home. Accessed 13 January, 2014.

Fagt S, Andersen LF, Anderssen SA, Becker W, Borodulin K, Fogelholm M, Groth MV, Gunnarsdottir I, Helakorpi S, Kolle E, Matthiessen J, Rosenlund-Sørensen M, Simonen R, Sveinsson T, Tammelin T, Thorgeirsdottir H, Valsta L, Trolle E (2012). Nordic monitoring of diet, physical activity and overweight. Validation of indicators. TemaNord 2011: 556. Nordic Council of Ministers.

Fox RJ, Crask MR, Kim J. Mail survey response rate. A meta-analysis of selected techniques for inducing response. Public Opinion Quartely. 1988;52:467-491. 
Fuchs M, Bossert D, Stukowski S. Response Rate and Nonresponse Bias - Impact of the Number of Contact Attempts on Data Quality in the European Social Survey. Bulletin of Sociological Methodology. 2013;117(1):26-45.

Giskes K, Avendano M, Brug, Kunst AF (2009). A systematic review of studies on socioeconomic inequalities in dietary intakes associated with weight gain and overweight/obesity conducted among European adults. Obes Rev; 11: 413-429.

Global BMI Mortality Collaboration (2016). Body-mass index and all-cause mortality: individualparticipant-data meta-analysis of 239 prospective studies in four continents. Lancet 388(10046):776-86.

Grimby G, Börjesson M, Jonsdottir IH, Schnohr P, Thelle DS, Saltin B. The "Saltin-Grimby Physical Activity Level Scale" and its application to health research. Scand J Med Sci Sports.

2015;25 Suppl 4:119-25.

Grotvedt L, Kuulasmaa K, Tolonen H, Heldal J, Graff-Iversen S. Sampling and Recruitment. In: Tolonen $\mathrm{H}$, Koponen $\mathrm{P}$, Aromaa A, et al., eds. Review of Health Examination Surveys in Europe. Vol B18/2008. Helsinki: National Public Health Institute; 2008. Available at http://urn.fi/URN:ISBN:978-951-740-843-1.

Gudlaugson JO, Magnusson KT and Johnsson SH (2014). Health and wellbeing of Icelanders 2012. Project report. Reykjavik: Directorate of Health.

Gudlaugsson JO, Magnusson KT and Jonsson SH (2014). Health and Wellbeing of Icelanders 2012. Project Report. Reykjavík: Directorate of Health. Retrieved 14.07.2015 from http://www.landlaeknir.is/tolfraedi-og-rannsoknir/rannsoknir/ heilsa-og-lidan-islendinga/

Gudlaugsson JO, Magnusson KT and Jonsson SH (2014). Health and Wellbeing of Icelanders 2012. Project Report. Reykjavík: Directorate of Health.

Gudlaugsson, JO (2015). Unpublished data from Health and Wellbeing of Icelanders 2007. Directorate of Health, Iceland.

Hallberg J, Österberg E (2014). Information on the nordic alcohol market 2014. Alko Inc, National Institute for Health and Welfare (THL), Helsinki, Finland.

Hara M, Sasaki S, Sobue T, Yamamoto S, Tsugane S. Comparison of cause-specific mortality between respondents and nonrespondents in a population-based prospective study: ten-year follow-up of JPHC Study Cohort I. Japan Public Health Center. J Clin Epidemiol. 2002;55(2):150-156.

Helakorpi S, Holstila AL, Virtanen S, Uutela A. Suomalaisen aikuisväestön terveyskäyttäytyminen ja terveys, kevät 2011 - Health Behaviour and Health among the Finnish Adult Population, Spring. 2011, National Institute for Health and Welfare, Helsinki.

Helakorpi S, Patja K, Prättälä R, Uutela A (2007). Health Behaviour and Health among the Finnish Adult Population, Spring 2006. National Public Health Institute.

Helldán A, Helakorpi S. Suomalaisen aikuisväestön terveyskäyttäytyminen ja terveys, kevät 2014 - Health Behaviour and Health among the Finnish Adult Population, Spring 2014. National Institute for Health and Welfare, Helsinki.

Helsedirektoratet (2009). Rapport: Fysisk aktivitet blant voksne og eldre i Norge. Resultater fra en kartlegging i 2008 og 2009 . Helsedirektoratet, Norway.

Howard B, Healy GN, Owen N (2012). Too much sitting - a health hazard. Diabetes Res Clin Pract 97(3):368-76. 
Inchley J, Currie D, Young T, Samdal O, Torsheim T, Augustson L, Mathison F, Aleman-Diaz A, Molcho M, Weber M, Barnekow V, eds (2016). Growing up unequal: gender and socioeconomic differences in young people's health and well-being. Health Behaviour in School-aged Children (HBSC) study: international report from the 2013/2014 survey. Copenhagen, WHO Regional Office for Europe (Health Policy for Children and Adolescents, NO. 7).

Jackson R, Chambless LE, Yang K, Byrne T, Watson R, Folsom A, Shahar E, Kalsbeek W. Differences between respondents and nonrespondents in a multicenter community-based study vary by gender ethnicity. The Atherosclerosis Risk in Communities (ARIC) Study Investigators. J Clin Epidemiol. 1996;49(12):1,441-1,446.

Johansen AMW (personal communication; 2015). Calculation based on data from the national dietary survey Norkost-3 study (2010-2011).

Johansen AMW (personal communication; 2015). Calculations based on data from the national dietary survey Ungkost-2000 study (2000).

Jonson SH, Gudlaugsson JO, Gylfason HF, Gudmundsdottir DG (2011). Health and Wellbeing of Icelanders 2007. Project Report. Reykjavík: Public Health Institute of Iceland.

Jonsson SH, Hedinsdottir M, Erlendsdottir RO, Gudlaugsson JO, Danielsdottir S and Reynisson JA (2013). School children body weight in the capital area. Results from Ískrá on children weight and height measurements 2003/4-2011/12. Reykjavik: Directorate of Health and Primary Health Care of the Capital Area. Report published on the Directorate of Health website: http://www.landlaeknir.is/servlet/file/ store93/item20002/Ofpyngd\%20Offita\%202-4-2013\%20(2).pdf

Jousilahti P, Salomaa V, Kuulasmaa K, Niemela M, Vartiainen E. Total and cause specific mortality among participants and non-participants of population based health surveys: a comprehensive follow up of 54372 Finnish men and women. J Epidemiol Community Health.

2005;59(4):310-315.

Kaartinen N \& Kanerva N (personal communication). Calculations based on FFQ questionnaire data from the National FINRISK 2007 and 2012.

Kalantar JS, Talley NJ. The effects of lottery incentive and length of questionnaire on health survey response rates: a randomized study. J Clin Epidemiol. 1999; 52 (11): 1,117-1,122.

Kjoller $\mathrm{M}$, Thoning $\mathrm{H}$. Characteristics of non-response in the Danish Health Interview Surveys, 1987-1994. Eur J Public Health. Oct 2005;15(5):528-535.

Knudsen AK, Hotopf M, Skogen JC, Overland S, Mykletun A. The health status of nonparticipants in a population-based health study: the Hordaland Health Study. Am J Epidemiol. Dec 1 2010;172(11):1,306-1,314.

Knudsen VK, Sørensen MR, Matthiessen J, Fagt S, Groth M (2012). Documentation report: Pilot Study 2010 [Internal report]. National Food Institute, Technical University of Denmark.

Kolle E, Stokke JS, Hansen BH, Anderssen S (2012). Rapport: Fysisk aktivitet blant 6-, 9- og 15åringer i Norge. Resultater fra en kartlegging i 2011. Helsedirektoratet, Norway.

Kopf ME, Scheib J, Blair J. The effect of alternative incentives on cooperation and refusal conversion in a telephone survey. ASA Proceedings of the Section on Survey Research Methods. 2000:1081-1085.

Kopra J, Härkänen T, Tolonen H, Karvanen J. Correcting for non-ignorable missingness in smoking trends. Stat. 2015; in press.

Korkeila K, Suominen S, Ahvenainen J, Ojanlatva A, Rautava P, Helenius H, Koskenvuo M. Non-response and related factors in a nation-wide health survey. Eur J Epidemiol. 2001;17(11):991-999. 
Larsen SB, Dalton SO, Schuz J, Christensen J, Overvad K, Tjønneland A, Johansen C, Olsen A. Mortality among participants and non-participants in a prospective cohort study. Eur J Epidemiol. 2012;27(11):837-845.

Lee IM, Shiroma EJ, Lobelo F, Puska P, Blair SN, Katzmarzyk PT. Effect of physical inactivity on major non-communicable diseases worldwide: an analysis of burden of disease and life expectancy. Lancet 2012; 380: 219-29.

Lundberg I, Thakker KD, Hallstrom T, Forsell Y. Determinants of non-participation, and the effects of non-participation on potential cause-effect relationships, in the PART study on mental disorders. Social Psychiatry and Psychiatric Epidemiology. 2005;40(6):475-483.

Lyhne N, Christensen T, Groth MV, Fagt S, Biltoft-Jensen A, Hartkopp H, Hinsch HJ, Matthiessen J, Møller A, Saxholt E, Trolle E (2005). Dietary habits in Denmark 2000-2002. Main results.. DFVF Pub. No. 11. Danish Institute for Food and Veterinary Research.

Maki P, Hakulinen-Viitanen T, Kaikkonen R, Koponen P, Ovaskainen M-L, Sippola R, Virtanen S, Laatikainen T and Late-working group (eds.) (2010). Child Health - Results of the LATE-study on growth, development, health, healthbehavior and growth environment. National Institute for Health and Welfare, Helsinki.

Matthiessen J (2015). Personal communication. Unpublished data from the Danish National Survey of Diet and Physical Activity 2011-2013. National Food Institute, Technical University of Denmark.

Matthiessen J, Stockmarr A. Flere overvægtige danske kvinder [More overweight Danish Women; article in Danish]. E-article nr. 2, DTU Food, September 2015, p. 1-10.

Moraeus L, Lissner L, Olsson L, Sjöberg A (2015). Age and time effects on children's lifestyle and overweight in Sweden. BMC Public Health 15:355.

Morgen CS, Rokholm B, Brixval CS, Andersen CS, Andersen GL, Rasmussen M, Andersen AMN, Due P, Sørensen TI (2013). Trends in prevalence of overweight and obesity in Danish infants, children and adolescents - are we still on a plateau? PLoS One 8: e6986o.

Nilsson A, Pearson M, Warensjö Lemming E, Kotova N (2014). Matvanor hos elever i åk 5 (Foodhabits among schoolchildren), National Food Agency, Sweden.

Nordic Council of Ministers (2006). A better life through diet and physical activity: Nordic Plan of Action on better health and quality of life through diet and physical activity. Copenhagen. Nordic Council of Ministers.

Nordic Council of Ministers (2006). Health, food and physical activity. Nordic Plan of Action on better health and quality of life through diet and physical activity. Copenhagen: Nordic Council of Ministers.

Nordic Council of Ministers (2014). Nordic Nutrition Recommendations 2012. Integrating nutrition and physical activity. Copenhagen. Nordic Council of Ministers.

Nordic Nutrition Recommendations 2004 (2004). Integrating nutrition and physical activity. 4 th ed. Nord 2004:13. Copenhagen: Nordic Council of Ministers.

Nordic Nutrition Recommendations 2012 (2014). Integrating nutrition and physical activity. 5th ed. Nord 2014:002. Copenhagen: Nordic Council of Ministers.

Nyholm M, Gullberg B, Råstam L, Lindblad U. What is the accurate prevalence of obesity in Sweden in the 21st century? Methodological experiences from the Skaraborg project. Obesity 2008;16:896-898. 
Ojala K (personal communication; 2015). Calculations based on data from the Health Behaviour in School-aged Children (HBSC) study in 2005-2006 and 2009-2010.

Olds T, Zumin S, Peneau S, Lioret S, Castetbon K, Bellisle, de Wilde J, Hohepa M, Maddison R, Lissner L, Sjöberg A, Zimmermann M, Aeberli I, Ogden C, Flegal K, Summerbell C (2011). Evidence that the prevalence of childhood overweight is plateauing: data from nine countries. Int $J$ Pediatr Obes 6:342-6o.

Pedersen AN, Christensen T, Matthiessen J, Knudsen VK, Sørensen MR, Biltoft-Jensen A, Hinsch HJ, Ygil K, Kørup K, Saxholt E, Trolle E, Søndergaard AB, Fagt S (2015). Dietary habits in Denmark 2011-2013. Main results. National Food Institute, Technical University of Denmark.

Pedersen AN, Christensen T, Matthiessen J, Knudsen VK, Sørensen MR, Biltoft-Jensen A, Hinsch HJ, Ygil K, Kørup K, Saxholt E, Trolle E, Søndergaard AB, Fagt S (2015). Dietary habits in Denmark 2011-2013. Main results. National Food Institute, Technical University of Denmark.

Peltonen M, Harald K, Männistö S, Saarikoski L, Lund L, Sundvall J, Juolevi A, Laatikainen T, AldénNieminen $\mathrm{H}$, Luoto $\mathrm{R}$, Jousilahti $\mathrm{P}$, Salomaa V, Taimi M, Vartiainen E. Kansallinen FINRISKI 2007terveystutkimus. Tutkimuksen toteutus ja tulokset: Taulukkoliite. (The National FINRISK 2007 Health Study. Methods and results: Tables). Publications of the National Public Health Institute, B 35/2008, Helsinki 2008.

Physical Activity Guidelines Advisory Committee (2008). Physical Activity Guidelines Advisory Committee Report, 2008. Washington, D.C: U.S. Department of Health and Human Services.

Public Health Agency (2006). Public Health data. The National Public Health Survey - Health on Equal Terms.

Public Health Agency (2011). Public Health data. The National Public Health Survey - Health on Equal Terms.

Public Health Agency (2011). Public Health data. The National Public Health Survey - Health on Equal Terms, Sweden.

Public Health Agency (2014). Public Health data. The National Public Health Survey - Health on Equal Terms.

Public Health Agency (2014). Public Health data. The National Public Health Survey - Health on Equal Terms, Sweden.

Rasmussen et al. (2012). Nordic Monitoring of diet, physical activity and overweight. First collection of data in all Nordic countries 2011. TemaNord 2012:552. Nordic Council of Ministers.

Rasmussen LB, Andersen LF, Borodulin K, Enghardt Barbieri H, Fagt S, Matthiessen J, Sveinsson, Trolle E (2012). Nordic monitoring of diet, physical activity and overweight. First collection of data in all Nordic Countries 2011. TemaNord 2012:552. Nordic Council of Ministers.

Rokholm B, Baker JL and Sorensen $\mathrm{TI}$ (2010). The levelling off of the obesity epidemic since the year 1999 - a review of evidence and perspectives. Obes Rev 11: 835-46.

Schweitzer M, Asch DA. Timing payments to subjects of mail surveys: cost-effectiveness and bias. J Clin Epidemiol. 1995;48(11):1,325-1,329.

Shahar E, Folsom AR, Jackson R. The effect of nonresponse on prevalence estimates for a referent population: insights from a population-based cohort study. Atherosclerosis Risk in Communities (ARIC) Study Investigators. Ann Epidemiol. 1996;6(6):498-506. 
Singer E, Van Hoewyk J, Gabler N, Raghunathan T, McGonagle K. The effect of incentives on response rates in interviewer-mediated surveys. Journal of Official Statistics. 1999;15(2):217-230.

Sjöberg A, Moreus L, Yngve A, Poortvliet E, Al-Ansari, Lissner L (2011). Overweight and obesity in a representative sample of Swedish schoolchildren - exploring the urban-rural gradient in Sweden. Obes Rev12;305-14.

Skretting A, Bye EK, Vedøy TF, Lund KE (2015). Rusmidler i Norge 2014. Report, The Norwegian Institute for Alcohol and Drug Research (SIRUS).

Sogaard AJ, Selmer R, Bjertness E, Thelle D. The Oslo Health Study: The impact of self-selection in a large, population-based survey. Int J Equity Health. 2004;3(1):3.

Stamatakis E, Hamer M, Dunstan DW (2011), Screen-Based Entertainment Time, All-Cause Mortality, and Cardiovascular Events Population-Based Study With Ongoing Mortality and Hospital Events Follow-Up. J Am Coll Cardiol 57(3):292-299.

Statistics Norway (2014). Health, care and social relations, survey on living conditions, 2012.

Steingrimsdottir L, Thorgeirsdottir H, Olafsdottir AS (2003). The Diet of Icelanders, Dietary Survey of the Icelandic Nutrition Council 2002. Main findings. The Public Health Institute of Iceland.

Thorgeirsdottir H, Valgeirsdottir H, Gunnarsdottir, I, Gisladottir E, Gunnarsdottir BE, Thorsdottir I, Stefansdottir J, Steingrimsdottir L (2011). The Diet of Icelanders 2010-11. Main findings. The Directorate of Health, the Icelandic Food and Veterinary Authority and the Unit of Nutrition Research (RIN) at the University of Iceland.

Thorp AA, Owen N, Neuhaus M, Dunstan DW (2011). Sedentary behaviors and subsequent health outcomes in adults a systematic review of longitudinal studies, 1996-2011. Am J Prev Med 41(2):207-15.

Tolonen $\mathrm{H}$, Aistrich A, Borodulin $\mathrm{K}$. Increasing health examination survey participation rates by SMS reminders and flexible examination times. Scand J Public Health. 2014;42(7):712-717.

Tolonen H, Helakorpi S, Talala K, Helasoja V, Martelin T, Prattala R. 25-year trends and sociodemographic differences in response rates: Finnish adult health behaviour survey. Eur J Epidemiol. 2006;21(6):409-415.

U.S. Department of Health and Human Services (2014). The Health Consequences of Smoking - 50 Years of Progress: A Report of the Surgeon General. Atlanta: U.S. Department of Health and Human Services, Centers for Disease Control and Prevention, National Center for Chronic Disease Prevention and Health Promotion, Office on Smoking and Health. (assessed 2016 Feb 23).

Une $\mathrm{H}$, Miyazaki M, Momose Y. Comparison of mortality between respondents and non-respondents in a mail survey. J Epidemiol. 2000;10(3):136-139.

Weeks MF, Kulka RA, Pierson SA. Optimal call scheduling for a telephone survey. Public Opinion Quarterly. 1987;51(4):540-549.

WHO (2011). Global status report on alcohol and health. Geneva: Switzerland. World Health Organization.

Wijnhoven TM, van Raaij JM, Spinelli A, Rito Al, Hovengen R, Kunesova M, Starc G, Rutter $H$, Sjöberg A, Petrauskiene A, O'Dwyer U, Petrova S, Farrugia Sant'angelo V, Wauters M, Yngve A, Rubana IM, Breda J (2013). WHO European Childhood Obesity Surveillance Initiative 2008: weight, height and body mass index in 6-9-year-old children. Pediatr Obes 8:79-97.

Virtanen V, Sirkia T, Jokiranta V. Reducing nonresponse by SMS reminders in mail surveys. Social Science Computer Review. 2007;25(3):384-395. 
World Health Organization (2000). Obesity: Preventing and Managing the Global Epidemic. Report of a WHO Consultation. Technical Report Series no. 894. Geneva: WHO.

World Health Organization (2002). Obesity: Preventing and Managing the Global Epidemic. Report of a WHO Consultation. Technical Report Series no. 894. Geneva: WHO.

World Health Organization (2009). Global health risks: mortality and burden of disease attributable to selected major risks. Geneva: Switzerland. World Health Organization. 


\title{
Sammenfatning
}

\author{
Baggrund og formål
}

Monitorering af kost, fysisk aktivitet og overvægt i Norden er en vigtig del af Den nordiske handlingsplan for bedre sundhed og livskvalitet gennem mad og fysisk aktivitet fra Nordisk Ministerråd. Nærværende rapport beskriver den anden dataindsamling i $2014 \mathrm{og}$ præsenterer status og udvikling fra 2011 til 2014. Resultaterne bliver evalueret i forhold til målene for 2011 og visionerne for 2021 i Den nordiske handlingsplan.

Formålet med Det nordiske monitoreringssystem er at indsamle data, der beskriver status og udvikling for kost, fysisk aktivitet og overvægt blandt voksne og børn i Norden. Derudover kan data bruges til at sammenligne resultaterne mellem de nordiske lande og sammenligne med anbefalinger for kost og fysisk aktivitet samt undersøge social ulighed i kost, fysisk aktivitet og overvægt. Endelig kan resultaterne evalueres i forhold til Den nordiske handlingsplans mål og visioner for at indikere, hvorvidt kost, fysisk aktivitet og overvægt ændres i ønskeværdig retning eller ej.

Fra 2014 er alkoholforbrug og rygevaner blandt voksne inkluderet i dataindsamlingen, hvilket gør det muligt også at sammenligne disse sundhedsindikatorer mellem de nordiske lande.

\section{Metode}

Data for kost, fysisk aktivitet og overvægt er blevet indsamlet i 2011 og 2014 blandt i alt 17.775 voksne i alderen 18-65 år og 4.949 børn i alderen 7-12 år i Danmark, Finland, Sverige, Island og Norge. En simpel tilfældig stikprøve af voksne og børn blev udtrukket fra de nationale populationsregistre i hvert af de fem nordiske lande. Data er indsamlet gennem telefoninterviews ved hjælp af et spørgeskema med validerede indikatorspørgsmål om kost, fysisk aktivitet og stillesiddende adfærd. Herudover er data indsamlet for højde og vægt (selvrapporteret) og enkelte sociodemografiske spørgsmål samt for voksnes alkoholforbrug og rygevaner. Data blev kontrolleret og oparbejdet af DTU Fødevareinstituttet, som også gennemførte de statistiske analyser. 
Data er vægtet i forhold til køn, alder og uddannelse. Brug af vægtede data introducerer mindre ændringer i forhold til tidligere publicerede resultater fra 2011.

Antallet af voksne deltagere i 2014 var 8.622. Den samlede svarprocent for voksne i hele Norden var 32\%. I 2014 deltog der 2.479 børn og den samlede svarprocent var $45 \%$. Blandt voksne var svarprocenten lavere i 2014 end 2011 (40\% vs. 32\%), mens der ingen ændring var blandt børn ( $45 \%$ vs. $45 \%$ ).

\section{Resultater}

Resultaterne præsenteres i fem kapitler om kost, fysisk aktivitet og stillesiddende adfærd, overvægt og svær overvægt, alkohol og rygning. Det efterfølgende kapitel giver et overblik over og en diskussion af resultaterne.

Der er lagt vægt på at beskrive hovedresultaterne i Norden og i forhold til køn, alder og uddannelse. Fokus i rapporten er lagt på at beskrive status og udvikling fra 2011 til 2014 for følgende nøglevariable: usund kost, inaktive, høj skærmtid i fritiden, overvægt eller svær overvægt og svær overvægt, og for voksne i 2014: rygere, daglige rygere, totalt alkohol forbrug og binge drinking den sidste måned. Nøglevariablene er udvalgt for at kunne evaluere målene for 2011 og visionerne for 2021 i Den nordiske handlingsplan for bedre sundhed og livskvalitet gennem kost og fysisk aktivitet. Variablene kan bidrage til at identificere de grupper i Norden, der formentligt vil drage mest fordel af en sundere livsstil og vægtstatus. Identifikationen af risikogrupper er et vigtigt redskab for de nordiske lande, da de gennem handlingsplanen for bedre sundhed og livskvalitet har forpligtet sig til at fremme en sundere livsstil i Norden.

Udvalgte data på landeniveau vurderes også i forhold til hvilket land, der har den mest eller mindst favorable position, hvad angår sund/usund adfærd og vægtstatus. Endelig er resultaterne evalueret i forhold til målene for 2011 og visionerne for 2021 i Den nordiske handlingsplan for bedre sundhed og livskvalitet.

\section{Status og udvikling i Norden fra 2011 til 2014}

Hovedresultaterne viser, at andelen af voksne i Norden med usund kost var højere end $20 \%$ og er øget fra 2011 til 2014, mens andelen med sund kost er faldet og kun var $10 \%$ i 2014. Hvorvidt kosten er sund eller usund er målt ved hjælp af en kostkvalitetsscore, som baseres på indtaget af frugt og grønt, fisk, fuldkornsbrød og fødevarer, som er rige på mættet fedt eller tilsat sukker. Blandt voksne i Norden er der kun set en gunstig udvikling i andelen med et højt indtag af fødevarer rige på tilsat sukker. Denne andel er også faldet i Danmark, Finland og Norge, men ikke i Sverige og Island. For de øvrige 
kostfaktorer har udviklingen ikke været gunstig, undtaget indtaget af frugt og grønt, som ikke ændrede sig i Norden, men som steg i Finland og Sverige, var vændret i Danmark og Island og faldt i Norge.

Hver tredje voksne i Norden var fysisk inaktiv og tre ud af ti brugte mere end 4 timer dagligt på stillesiddende skærmtid i fritiden. Derudover var forekomsten af overvægt/svær overvægt og svær overvægt høj blandt voksne. Således er det i dag næsten lige så almindeligt at være overvægtig/svært overvægtig som normalvægtig i Norden. Ydermere er stigningen i forekomsten af svær overvægt og i andelene med usund kost, meget inaktive eller høj skærmtid i fritiden blandt voksne bekymrende. Dog ses der også en lovende stigning i andelen af meget fysisk aktive, hvilket kunne tyde på en stigende polarisering af det fysiske aktivitetsmønster blandt voksne i Norden.

Udviklingen i alkoholforbruget og rygning kan ikke beskrives, da disse data kun er indsamlet i Det nordiske monitoreringssystem i 2014. Blandt voksne i Norden er det gennemsnitlige alkoholindtag 1,7 gange om ugen og andelen, der drikker fem eller flere genstande ved én lejlighed er $45 \%$ (binge-drinking). Andelen af rygere er $21 \%$ og andelen af daglige rygere er $14 \%$.

Resultaterne viser, at sundhedsadfærd og vægtstatus, dvs. kost, stillesiddende adfærd, rygning, alkohol, overvægt og svær overvægt er mindre gunstig blandt mænd end blandt kvinder i Norden.

Blandt børn i Norden ses der ingen ændringer i kost, fysisk aktivitet og overvægt fra 2011 til 2014. Ligesom for voksne ses der en gunstig udvikling i Norden i andelen af børn med et højt indtag af fødevarer rige på tilsat sukker, selvom der ikke ses ændringer i de fem nordiske lande. Derudover steg indtaget af frugt og grønt og fisk i Norden.

Selvom det er lovende, at udviklingen blandt børn i Norden ikke går i samme retning som for voksne, så er de høje andele af børn med usund kost, som er fysisk inaktive eller er overvægtige i 2014 bekymrende. Skønt andelen af børn med usund kost $\operatorname{var} 15 \%$, så var andelen med en sund kost kun 10\%. Andelen af fysisk inaktive var ca. $60 \%$ og derfor meget høj. Også forekomsten af overvægt eller svær overvægt er relativ høj (15\%) og en stagnation i udviklingen er tidligere blevet efterfulgt af en stigning.

Kønsforskelle i sundhedsadfærd og vægtstatus er ikke lige så tydelige blandt børn som blandt voksne i Norden. Dog er der flere drenge end piger med usund kost og med stillesiddende adfærd. Derimod er andelen af fysisk inaktive højere blandt piger end blandt drenge.

\section{Udfordringer ifolkesundheden i de nordiske lande}

Sammenligning mellem de nordiske lande viser, at hvert land er udfordret med ugunstige befolkningsniveauer og/eller udvikling i sundhedsadfærd og/eller vægtstatus 
blandt voksne og/eller børn. Danmark er udfordret med ugunstige ryge- og alkoholvaner blandt voksne. Stillesiddende adfærd kan være en stigende folkesundhedsudfordring blandt voksne i Finland, eftersom stigningen i høj skærmtid i fritiden er bekymrende. Befolkningsniveauet og udviklingen i andelen med usund kost i Sverige er bekymrende, især blandt voksne. Derudover udgør den høje andel inaktive børn i Sverige et folkesundhedsproblem, selvom der ikke er set ændringer fra 2011 til 2014. Niveauet af og udviklingen i andelen af både overvægtige og svært overvægtige blandt voksne i Island er et stort folkesundhedsproblem. I Norge vækker den høje andel af inaktive voksne bekymring, selvom der ikke set ændringer fra 2011 til 2014.

Der er således fortsat plads til forbedring i alle nordiske lande indenfor bestemte områder og udviklingen blandt voksne har primært været ugunstig eller uændret i de enkelte lande, hvorimod udviklingen blandt børn har været gunstig eller vændret.

\section{Evaluering af målene for 2011 og visionerne for 2021 for kost, fysisk aktivitet og overvægt}

Evaluering af hovedresultaterne i forhold til målene for 2011 og visionerne for $2021 \mathrm{i}$ Den nordiske handlingsplan for bedre sundhed og livskvalitet afspejler status og udviklingen i Norden. Planen sætter mål for en gunstig udvikling i forhold til kost, fysisk aktivitet og overvægt, mens visionerne fokuserer på at nå et konkret niveau i befolkningen i 2021.

Målet vedrørende tilsat sukker: "Indtaget af tilsat sukker er mindsket" er opfyldt blandt voksne og børn i Norden.

Blandt børn er fire andre mål blevet opfyldt: "Indtaget af frugt og grønt er steget", "Indtaget af fisk er steget", "Stigningen i andelen af fysisk inaktive er stoppet eller i bedste fald vendt" og "Den fortsatte stigning i andelen af overvægtige og svært overvægtige børn er stoppet eller i bedste fald vendt".

Derudover er målene om social ulighed i kost, fysisk aktivitet og overvægt tilsyneladende også opfyldt, idet der ses et fald eller ingen ændring i social ulighed blandt voksne og børn indenfor alle tre områder, undtaget for børn med hensyn til kost. Dog er den sociale ulighed primært faldet, fordi sundhedsadfærd og vægtstatus er blevet dårligere i den højeste uddannelsesgruppe.

Den eneste vision som pt. er opfyldt både blandt voksne og børn i Norden er indtaget af fuldkornsbrød. Visionen om indtaget af fuldkornsbrød bør dog revideres, idet visionen i sin nuværende ordlyd er afhængig af indtaget af hvidt brød. 


\section{Konklusion}

I Det nordiske monitoreringssystem er der indsamlet data i 2011 og 2014. I denne treårige periode er der sket signifikante ændringer i Norden, om end nogle af ændringerne er små. Visse af ændringerne kan dog skyldes naturlig variation som følge af bl.a. lav svarprocent. Baseret på to dataindsamlinger med tre års mellemrum er det derfor for tidligt at drage for håndfaste konklusioner om udviklingen i Norden. Dataindsamlingen bør gentages før udviklingstendenser kan tolkes mere sikkert.

Styrken ved Det nordiske monitoreringssystem er sammenligneligheden af data mellem undersøgelsesår, alders og uddannelsesgrupper og mellem de enkelte lande. Andre nationale undersøgelser kan ikke tilvejebringe denne sammenlignelighed, da målemetoder, undersøgelsesår og alders- og uddannelsesgrupper varierer. Men før en tredje dataindsamling påbegyndes, er der behov for at forbedre svarprocenten i flere nordiske lande. Derudover bør visse nøglevariable, der anvendes i evalueringen af målene og visionerne i Den nordiske handlingsplan revideres kritisk.

Blandt voksne i Norden vil nogle af visionerne for 2021 være mulige at opfylde, bl.a. visionen om indtaget af tilsat sukker, befolkningsniveauet af fysisk aktive og forskellene i social ulighed i fysisk aktivitet og overvægt, mens andre visioner angående indtaget af frugt og grønt, fisk og mættet fedt, befolkningsniveauet af overvægt og svær overvægt og forskellen i social ulighed i kost vil være svære at opfylde i 2021.

Blandt børn i Norden er befolkningsniveauet af svær overvægt den eneste vision, som det er muligt at opfylde i 2021, mens visionerne om indtaget af tilsat sukker, befolkningsniveauet af fysisk aktive og visionerne om indtaget af frugt og grønt, fisk og mættet fedt samt befolkningsniveauet af overvægt og forskellen i social ulighed $i$ kost vil være svære at opfylde.

Samlet set viser resultaterne, at udviklingen fra 2011 til 2014 i kost, fysisk aktivitet og overvægt går i den forkerte retning blandt voksne i Norden, mens udviklingen blandt børn er mere lovende, men med plads til forbedringer. En tredje dataindsamling vil vise om de udviklingstendenser, der er fundet vil fortsætte eller om sundhedsfremmende initiativer indenfor kost, fysisk aktivitet og overvægt kan føre til en sundere livsstil og vægtstatus blandt voksne og børn i Norden. Mænd, 45-65-årige og kort uddannede er særligt relevante grupper at målrette folkesundhedsinitiativer mod for at opfylde den fælles nordiske ambition om at sikre bedre sundhed og livskvalitet og lighed for alle nordiske borgere. 



\section{Appendix A: Questionnaire}

The questionnaire used in adults (18-65y) and children (7-12 y) are shown in the following pages. The questionnaire is developed to be used as computer assisted telephone interview (CATI) and therefore the look of the questionnaire is dependent on the system used for CATI.A preliminary suggestion on coding is shown. Text in bold is read out aloud, while text in Italics normally is information for the interviewer. 
Figure 11: Questionnaire Adult

Diet and physical activity of Nordic adults

English Master questionnaire August 2014

Date of interview

Name of interviewer, identification number of repondent etc.

Q0 Registration of the sex of the interviewed person

Male ......

Female

Q1 A. What is your date of birth ?
$1 \mathrm{~A}$ Month
L
1B Year Lـــــــ (19

January $=01$

February $=02$

May $=03$

Etc.

Q2. What is your education ${ }^{1}$ ?

Basic education

10 years or less

Vocational education/ Practical - Upper secondary education approx. 1-3 years after Basic education

Theoretical Upper Secondary education

approx. 2-3 years after Basic education

Short higher education - not vocational or practical

approx. 1-2 years after basic or secondary education

Medium higher

approx. 3-4 years after secondary education

Long higher education

approx. 5+ years after secondary education

1

$\square 2$

3

4

5

6

${ }^{1}$ Combined school and further education. The education has to be completed. 


\section{Q3. Which description explains best where you live?}

The Capital and suburb.

A large city (more than 50.000 inhabitants)

A city of between $20.000-49.999$ inhabitants...

A town between $1000-19.999$ inhabitants.

Country side

Do not know

Q4. Do you live together with anyone? (multiple responses possible)

$$
1=\text { Yes, } 0=\text { No }
$$

4.1Yes, with spouse/partner or cohabite

4.2 Yes, with mother/father/parents

4.3 Yes, with kids living at home (kids $<18 \mathrm{y}$ )

4.4 Yes, with kids living at home $(\geq 18 \mathrm{y})$

4.5 Yes, with others

4.6 No I live alone

$1=$ Yes, else 0

$\square 1=$ Yes, else 0

$\square 1=$ Yes, else 0

$\square 1=$ Yes, else 0

$1=$ Yes, else 0

$1=$ Yes, else 0

4.6 (Single Response only) If $4.6=1 \rightarrow$ go to question 6

Q5. How many people live in your household including yourself?

number of persons in the household

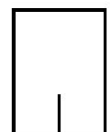

(Must be at least 2)

Q6. How tall are you?

L. $\rfloor_{\mathrm{cm}}$.

Do not know

Refuse

997

Q7. How much do you weigh? (for pregnant women: the weight before pregnancy)

Lـ, لـ $\mathrm{kg}$

Do not know 
The following questions regard what you usually eat. Please keep the last 12 months in mind when you respond to the questions. You have to keep in mind what you eat most often

8 What type of spread/grease do you usually put on your bread? If you use more than one kind, respond to what you use the most.

If the respondent mentions butter, ask if he/she means butter or Kærgården/similar.

If the respondent is in doubt whether she/he uses margarine or vegetable margarine, ask if the grease comes in paper wrapping or in a container (if national relevance)

$1 \square$ Butter

$2 \square$ Oil-butter spereads, Kærgården, Bakkedal, Marklyst, Mælkebøtte, Butter Bar

${ }_{3} \square$ Vegetable margarine $60-80 \%$

${ }_{4} \square$ Low fat margarine ('Lätta', 'Becel', 38\% fat)

$5 \square$ 'Becel Pro-Activ'

$6 \square$ Margarine $70-80 \%$

$7 \square$ Fat (pig or duck, coco)

$8 \square$ Do not use spread/grease/fat on bread

${ }_{98} \square$ Do not know

9 What type of fat, eg. butter, margarine or oil, do you usually use for domestic cooking? If you use more than one kind, respond regarding to what you use most.

If the respondent mentions butter, ask if he/she means butter or 'Kærgården'/ similar.

If the respondent is in doubt whether she/he uses margarine or vegetable margarine, ask if the grease comes in a paper wrapper or in a container (if national relevance)

$01 \square$ Butter

$02 \square$ Oil-butter spreads, Kærgården, Bakkedal, Marklyst, Mælkebøtte, Lurpak butter bar

$03 \square$ Frying or baking margarine $70-80 \%$ fat

$04 \square$ Vegetable margarine $60-80 \%$ fat

$05 \square$ Fluid margarine, oil-margarine (eg 'Becel', 'Lise')

$06 \square$ Oil (eg. rapeseed oil, olive oil, corn oil, sunflower oil, grapeseed oil, salad oil etc)

$07 \square$ Use a mixture of oil and butter/Kærgården

$08 \square$ Fat (pig, duck)

$09 \square$ Do not use spread/grease/fat for cooking

$10 \square$ We do not cook/prepare food in our household

$11 \square$ Kasvisterolimargariinia (esim. Becel ProActivem Benecol (only Finland)

$98 \square$ Do not know 
10 How many slices of bread do you eat per day or per week?

Answer according to slice/piece $/ 1 / 2$ roll

Grain bread also includes wholemeal bread, full grain bread and might carry the wholegrain label. Rolls are also considered bread.

Slice $/$ piece $/ 1 / 2$ roll

A.per day or B.per week

10.1

Rye Bread ((Not to be asked in Sweden))

How many slices of rye bread do you eat?

Never eat 97

Do not know 98

10.1a per day

10.1b per week

10.2

White Bread or Wholegrain (not rye bread):

How many slices of whole grain bread, with grain do you eat?

Never eat 97

Do not know 98

10.2a per day

10.2b per week

10.3

How many slices of white bread, toastbread, ciabbata do you eat?

Never eat 97

Do not know 98

10.3a per day

10.3b per week

10.4

How many slices of hard bread do you eat?

Never eat 97

Do not know 98

10.4a per day

$10.4 \mathrm{~b}$ per week

Code 99 if unanswered 
11 How often do you eat fruit and vegetables during a day, a week or a month. If you do not eat fruit and vegetables every day, please think about how often you eat it in a week or in a month. Think about the last 12 months when you respond.

Please respond to both sub questions, but only one response (cross, $X$ ) on each row

Do not count small portions, eg. a slice of cucumber on bread, parsley as decoration, berries on cake etc.

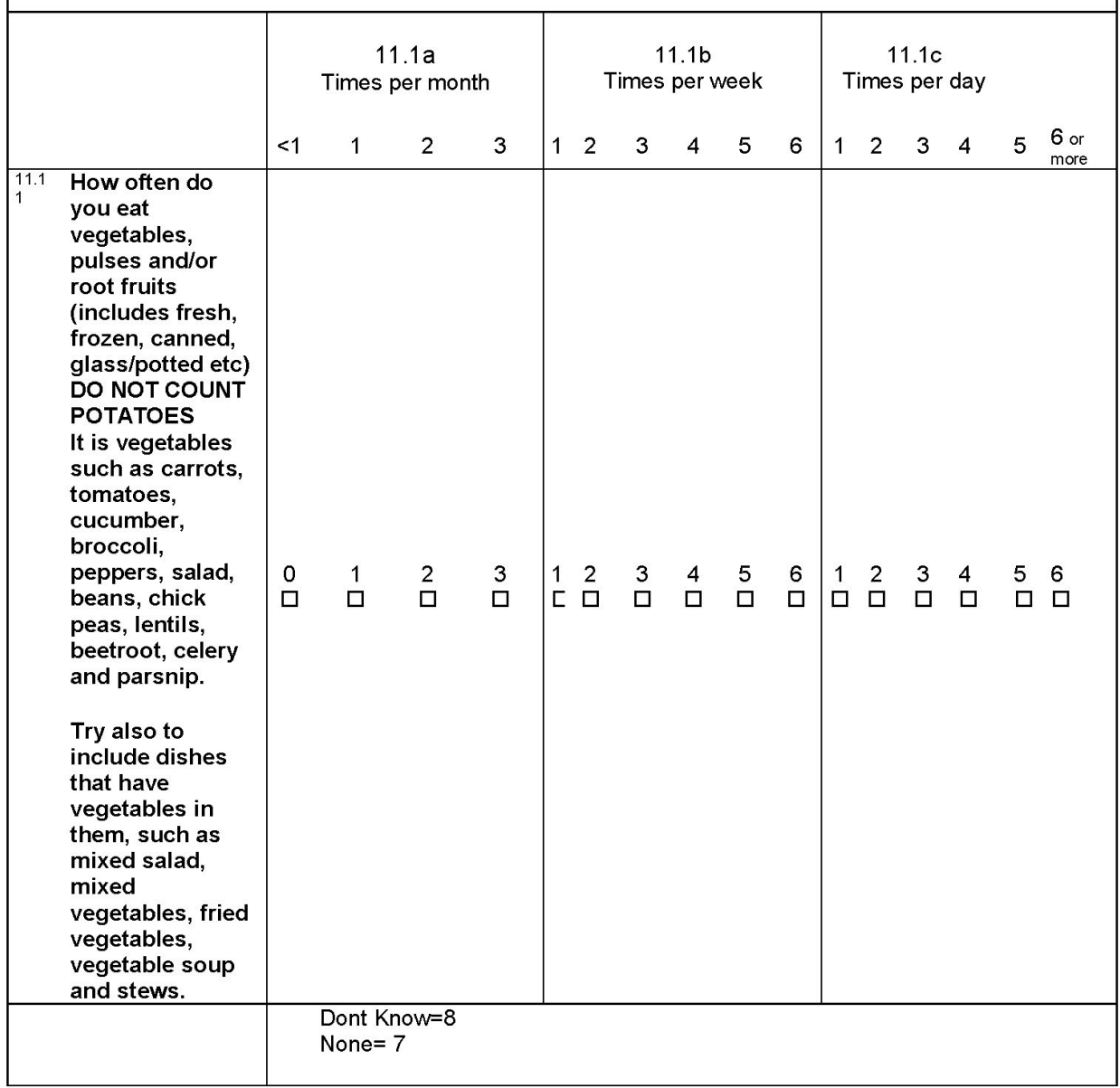




\begin{tabular}{|c|c|c|c|c|c|c|c|c|c|c|c|c|c|c|c|}
\hline \multirow[t]{4}{*}{12} & \multirow{2}{*}{\multicolumn{15}{|c|}{$\begin{array}{l}\text { Now I will ask you how you eat and drinks a selection of food. Please consider the } \\
\text { past } 12 \text { months when you answer these questions. } \\
\text { Please answer all questions, but only cross one box on each row }\end{array}$}} \\
\hline & & & & & & & & & & & & & & & \\
\hline & & \multicolumn{4}{|c|}{$\begin{array}{c}\text { A } \\
\text { Times per month }\end{array}$} & \multicolumn{6}{|c|}{$\begin{array}{c}\text { B } \\
\text { Times per week }\end{array}$} & \multicolumn{4}{|c|}{$\begin{array}{c}\text { C } \\
\text { Times per day }\end{array}$} \\
\hline & & $<1^{*}$ & 1 & 2 & 3 & 1 & 2 & 3 & 4 & 5 & 6 & 1 & 2 & 3 & $\begin{array}{l}4 \text { or } \\
\text { mor }\end{array}$ \\
\hline & $\begin{array}{l}\text { How often do you } \\
\text { eat/drink? }\end{array}$ & 0 & 1 & 2 & 3 & 1 & 2 & 3 & 4 & 5 & 6 & 1 & 2 & 3 & 4 \\
\hline 12.01 & $\begin{array}{l}\text { French fries, } \\
\text { roasted/fried } \\
\text { potatoes }\end{array}$ & $\square$ & $\square$ & $\square$ & $\square$ & $\square$ & $\square$ & 口 & $\square$ & $\square$ & $\square$ & $\square$ & $\square$ & $\square$ & $\square$ \\
\hline 12.02 & $\begin{array}{l}\text { Fish and shellfish } \\
\text { as main course }\end{array}$ & $\square$ & 口 & $\square$ & $\square$ & $\square$ & 口 & 口 & $\square$ & $\square$ & $\square$ & $\square$ & $\square$ & $\square$ & $\square$ \\
\hline 12.03 & $\begin{array}{l}\text { Sausage as a main } \\
\text { course }\end{array}$ & $\square$ & 口 & $\square$ & $\square$ & $\square$ & $\square$ & 口 & $\square$ & $\square$ & $\square$ & $\square$ & $\square$ & $\square$ & $\square$ \\
\hline 12.04 & $\begin{array}{l}\text { Chocolate and/or } \\
\text { candy }\end{array}$ & ㅁ & 口 & $\square$ & $\square$ & $\square$ & 口 & 口 & $\square$ & $\square$ & $\square$ & $\square$ & $\square$ & $\square$ & $\square$ \\
\hline 12.05 & $\begin{array}{l}\text { Cake, biscuits, tart } \\
\text { etc. }\end{array}$ & $\square$ & $\square$ & $\square$ & $\square$ & $\square$ & $\square$ & $\square$ & $\square$ & $\square$ & $\square$ & $\square$ & $\square$ & $\square$ & $\square$ \\
\hline 12.06 & $\begin{array}{l}\text { Full fat cheese ( } 45- \\
60+\text { or } 24-44 \% \text { fat) }\end{array}$ & $\square$ & $\square$ & $\square$ & $\square$ & $\square$ & $\square$ & 口 & $\square$ & $\square$ & $\square$ & $\square$ & $\square$ & $\square$ & $\square$ \\
\hline 12.07 & $\begin{array}{l}\text { Low fat/sugar-free } \\
\text { fizzy drinks, } \\
\text { cordial, ice-tea, } \\
\text { light or sugar free } \\
\text { drinks }\end{array}$ & $\square$ & $\square$ & $\square$ & $\square$ & $\square$ & $\square$ & $\square$ & $\square$ & $\square$ & $\square$ & $\square$ & $\square$ & $\square$ & $\square$ \\
\hline 12.08 & $\begin{array}{l}\text { Normal fizzy drinks, } \\
\text { cordial, ice-tea }\end{array}$ & $\square$ & $\square$ & $\square$ & $\square$ & $\square$ & $\square$ & $\square$ & $\square$ & $\square$ & $\square$ & $\square$ & $\square$ & $\square$ & $\square$ \\
\hline 12.09 & $\begin{array}{l}\text { Energy drinks, red } \\
\text { bull, cult etc. }\end{array}$ & $\square$ & 口 & $\square$ & $\square$ & $\square$ & $\square$ & 口 & $\square$ & $\square$ & $\square$ & $\square$ & $\square$ & $\square$ & $\square$ \\
\hline 12.10 & $\begin{array}{l}\text { Fruit juice or } \\
\text { Vegetable Juice }\end{array}$ & $\square$ & 口 & $\square$ & $\square$ & $\square$ & 口 & 口 & $\square$ & $\square$ & $\square$ & $\square$ & $\square$ & $\square$ & $\square$ \\
\hline & & \multicolumn{14}{|c|}{$\begin{array}{l}\text { Dont Know }=8 \\
\text { None }=7\end{array}$} \\
\hline
\end{tabular}




\section{The next questions regard your physical activity}

[Comment: The interviewer reads two to three examples of physical activities. Additional examples are in italics and if the respondents have difficulties in answering, the interviewer can add some of these additional examples on request. Generally the interviewer does not read the text in italics but can use the text as a reminder or can read it on request.]

I am going to ask you about your physical activity during the last 7 days. Your information is important even if you have not been physically active.

13a. Do you work, attend school or university?

$1 \square$ Yes $5 \square$ No

Filter:

If $\mathrm{No}(5)$ in question $13 a \rightarrow$ go to question 14

$13 \mathrm{~b}$ Which one of the following descriptions best fits your occupation, or school hours?

Do not include travel to and from work or school

(1) Mostly sedentary work like office work

Additional examples are cashier in a store or in a bank, and light manual work

(2) Work that requires a lot of walking like teaching Additional examples are shop assistant, light industrial work

(3) Work that requires a lot of walking and lifting, like a nurse 3 Additional examples are heavy industrial work

(4) Heavy manual labour like heavy construction 4 Additional examples are heavy farm work, heavy forestry 
[Question 14 (moderate or harder activity)]

Next, I am going to ask you about all physical activity during your leisure time and active transportation e.g. commuting to and from work or school. include $P A$ while running errands. Include all activity where the physical effort is moderate or harder, that is, you should include both moderate and vigorous activity. This kind of activity accelerates heart rate and breathing. Examples are brisk walking, running and heavy gardening.

Additional examples are Nordic walking, bicycling, and golf; these examples can be country specific.

Q14

During the last 7 days, how much time in total did you spend in physical activity where the physical effort was moderate or harder and lasted for at least $10 \mathrm{~min}$ each time? Estimate to the nearest half an hour.

The interviewer can help the respondent to narrow down the answer to the nearest half hour. It is important to know if physical activity is less or more than $150 \mathrm{~min}(21 / 2 \mathrm{~h})$ and if it is more or less than 300 min (5 h)

Hours.

Minutes.

DK (Dont Know)

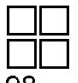

[Question 15 (vigorous activity)]

Q15

Next, I am going to ask you how much of the physical activity you indicated in the last question, was vigorous. This kind of activity causes substantial increase in heart rate and sweating, as well as rapid breathing that makes it difficult to talk. Examples are running or playing soccer Additional examples are fast bicycling, badminton or tennis, and cross-country skiing; these examples can be country specific.

During the last 7 days, how much time in total did you spend during leisure time in vigorous physical activity that lasted for at least $\mathbf{1 0}$ min each time? Estimate to the nearest half hour.

The interviewer can help the respondent to narrow down the answer to the nearest half hour. It is important to know if physical activity is less or more than $75 \mathrm{~min}$ ( $1 \mathrm{~h}$ and 15 $\mathrm{min})$ and if it is more or less than $150 \mathrm{~min}\left(2 \frac{1}{2} \mathrm{~h}\right)$

Hours.

Minutes.

DK (Dont Know)

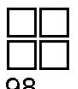

[Question 16a (Sedentary time: TV watching)]

Interviewer: During the last 7 days, how much time per day on average did you spend sitting and watching TV during your leisure time? Estimate it to the nearest half hour. Include videos, DVD and console games (PlayStation, Xbox, etc) played on TV screen.

The interviewer can help the respondent to narrow down the answer to the nearest half hour. It is important to know if average time is less or more than 1 hour and if it is more orless than $2 \frac{1}{2}$ hours

Hours......

Minutes.

DK (Dont Know)

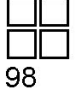




\begin{tabular}{|c|c|c|c|c|c|c|c|c|c|c|c|c|c|c|c|c|c|}
\hline \multirow[t]{3}{*}{$\begin{array}{l}\text { Question } \\
18\end{array}$} & \multicolumn{17}{|c|}{$\begin{array}{l}\text { Now I will ask you how often you drink a selection of beverages. Please consider the past } 12 \\
\text { months when you answer these questions. } \\
\text { Please answer all questions, but only cross one box on each row }\end{array}$} \\
\hline & & \multirow[t]{2}{*}{ Never } & \multicolumn{2}{|c|}{$\begin{array}{l}\text { Times per } \\
\text { year }\end{array}$} & \multicolumn{3}{|c|}{$\begin{array}{l}\text { Times per } \\
\text { month }\end{array}$} & \multicolumn{6}{|c|}{ Times per week } & \multicolumn{4}{|c|}{ Times per day } \\
\hline & & & $1-5$ & $6-11$ & 1 & 2 & 3 & 1 & 2 & 3 & 4 & 5 & 6 & 1 & 2 & 3 & $\begin{array}{l}4 \text { or } \\
\text { more }\end{array}$ \\
\hline 11 & Beer* & $\square$ & $\square$ & $\square$ & $\square$ & $\square$ & $\square$ & $\square$ & $\square$ & $\square$ & $\square$ & $\square$ & $\square$ & $\square$ & $\square$ & $\square$ & $\square$ \\
\hline 12 & Wine & $\square$ & $\square$ & $\square$ & $\square$ & $\square$ & $\square$ & $\square$ & $\square$ & $\square$ & $\square$ & $\square$ & $\square$ & $\sqcup$ & $\square$ & $\square$ & $\square$ \\
\hline 13 & Spirits $\star \star$ & $\square$ & $\square$ & $\square$ & $\square$ & $\square$ & $\square$ & $\square$ & $\square$ & $\square$ & $\square$ & $\square$ & $\square$ & $\square$ & $\square$ & $\square$ & $\square$ \\
\hline
\end{tabular}

${ }^{*}$ Including cider with $4-5 \%$ alcohol content

**Including long drinks, alcopops

Questi During the previous month, how many times have you had five or more units of on 18 alcohol at a single occasion?

a

Number of times........ $\square$

A unit of alcohol could be:

1 beer $=1$ unit

1 alcopop $=1$ unit

1 glass of wine $=1$ unit a bottle of wine $=6$ unit

1 shot of liquor or spirit $=1$ unit

Question Do you currently smoke tobacco on a daily basis, less than daily or not at all?

19

\begin{tabular}{|l|c|c|l}
\hline Daily & $\square$ & 1 & End section \\
Less than daily & $\square$ & 2 & Go to 19a \\
Not at all & $\square$ & 3 & Go to 19b \\
Don't know & $\square$ & 8 & End section \\
\hline
\end{tabular}

Question Have you smoked tobacco daily in the past?

19 a 


\begin{tabular}{|l|c|c|l|}
\hline Yes & $\square$ & 1 & End section \\
No & $\square$ & 2 & End section \\
Don't know & $\square$ & 8 & End section \\
\hline
\end{tabular}

\begin{tabular}{|l|c|c|c|}
\hline $\begin{array}{l}\text { Question In the past, have you smoked tobacco on a daily basis, less than daily or not at all? } \\
19 \mathbf{~ b}\end{array}$ & $\square$ & 1 & \\
\hline Daily & $\square$ & 2 & \\
Less than daily & $\square$ & 3 & \\
Not at all & $\square$ & 8 & \\
Don't know & & $\square$ \\
\hline
\end{tabular}

\begin{tabular}{|l|l|l|l|}
\hline $\begin{array}{l}\text { Question } \\
20\end{array}$ & $\begin{array}{l}\text { Do you currently use snuff on a daily basis, less than daily or not at all? } \\
\text { (not relevant for Denmark) }\end{array}$ \\
\hline Daily & $\square$ & 1 & End section \\
Less than daily & $\square$ & 2 & Go to 20a \\
Not at all & $\square$ & 3 & Go to 20b \\
Don't know & $\square$ & 8 & End section \\
\hline
\end{tabular}

\begin{tabular}{|l|c|c|l|}
\hline $\begin{array}{l}\text { Question } \\
20 a\end{array} \quad \begin{array}{l}\text { Have you used snuff daily in the past? } \\
\text { (not relevant for Denmark) }\end{array}$ \\
\hline Yes & $\square$ & 1 & End section \\
No & $\square$ & 2 & End section \\
Don't know & $\square$ & 8 & End section \\
\hline
\end{tabular}

Question In the past, have you used snuff on a daily basis, less than daily or not at all? 20b (not relevant for Denmark)

\begin{tabular}{|l|c|c|c|}
\hline Daily & $\square$ & 1 & \\
Less than daily & $\square$ & 2 & \\
Not at all & $\square$ & 3 & \\
Don't know & $\square$ & 8 & \\
\hline
\end{tabular}


Figur 12: Questionnaire Children

\section{Diet and physical activity of Nordic children}

English Master questionnaire August 2014

(no changes from 2011-version)

Date of interview

Name of interviewer, identification number of repondent etc.

Q0 Registration of the sex of the interviewed person

Male

Female

Q1 A. What is your child's date of birth ?

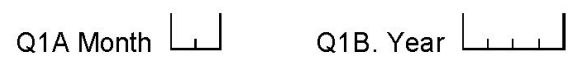

January $=01$

February $=02$

May $=03$

Etc.

Q1C. What is your child's sex?

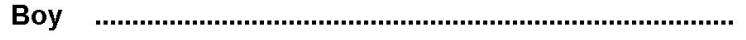

Girl

Q1C open : What is the child's pet name? Write here:

Q1D. Are you the child's:

Mother/Stepmother .

Father/Stepfather

Other, write the relationship here:

B. 
Q2. What is your education ${ }^{1}$ ?

$\begin{array}{lr}\text { Basic education } & \square 1 \\ \text { 10 years or less } & \\ \begin{array}{l}\text { Vocational education/ Practical - Upper secondary education } \\ \text { approx. 1-3 years after Basic education }\end{array} & \square 2 \\ \begin{array}{l}\text { Theoretical Upper Secondary education } \\ \text { approx. 2-3 years after Basic education }\end{array} & \square 3 \\ \begin{array}{l}\text { Short higher education - not vocational or practical } \\ \text { approx. 1-2 years after basic or secondary education }\end{array} & \square 4 \\ \begin{array}{l}\text { Medium higher } \\ \text { approx. 3-4 years after secondary education }\end{array} \\ \begin{array}{l}\text { Long higher education } \\ \text { approx. 5+ years after secondary education }\end{array}\end{array}$

Q3. Which description explains best where you live?

(Place only one cross)

The Capital and suburb.

1

A large city (more than 50.000 inhabitants)

A city of between $20.000-49.999$ inhabitants...

A town between $1000-19.999$ inhabitants...

Country side

Do not know

\footnotetext{
${ }^{1}$ Combined school and further education. The education has to be completed.
} 
Q4. Do you live together with anyone? (multiple responses possible) $1=$ Yes, $0=\mathrm{No}$

4.1Yes, with spouse/partner or cohabite

4.2 Yes, with mother/father/parents

$1=$ Yes, else 0

4.3 Yes, with kids living at home (kids $<18$ y)

4.4 Yes, with kids living at home $(\geq 18 \mathrm{y})$

$1=$ Yes, else 0

4.5 Yes, with others

$1=$ Yes, else 0

$1=$ Yes, else 0

4.6 No I live alone

$1=$ Yes, else 0

4.6 (Single Response only) If $4.6=1 \rightarrow$ go to question 6

Q5. How many people live in your household including yourself?

number of persons in the

household

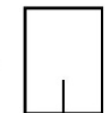

(Must be at least 2) 
Q6. How tall is your child?

$$
\text { L. } \mathrm{L} \text { cm. }
$$

Do not know

No response

Q7. How much does your child weigh?<smiles>CCCC(C)CC</smiles>

Do not know 998

No response 997

The following questions regard what your child usually eat. Please keep the last 12 months in mind when you respond to the questions. You have to keep in mind what your child eats most often (if national relevance)

Q What type of spread/grease do your child usually put on his/hers bread? If your child use more than 8 one kind, respond to what your child uses the most.

If the respondent mentions butter, ask if he/she means butter or Kærgården/similar.

If the respondent is in doubt whether the child uses margarine or vegetable margarine, ask if the grease comes in paper wrapping or in a container

$1 \square$ Butter

$2 \square$ Oil-butter spreads, Kærgården, Bakkedal, Marklyst, Mælkebøtte, Butter Bar

$3 \square$ Vegetable margarine $60-80 \%$

$4 \square$ Low fat margarine ('Lätta', 'Becel', 38\% fat)

$5 \square$ 'Becel Pro-Activ'

$6 \square$ Margarine $70-80 \%$

$7 \square$ Fat (pig or duck)

${ }_{8} \square$ Do not use spread/grease/fat on bread

$98 \square$ Do not know 
9 What type of fat, eg. butter, margarine or oil, do you usually use for domestic cooking? If you use more than one kind, respond regarding to what you use most. (if national relevance)

If the respondent mentions butter, ask if he/she means butter or 'Kærgården'/ similar.

If the respondent is in doubt whether she/he uses margarine or vegetable margarine, ask if the grease comes in a paper wrapper or in a container

\section{$01 \square$ Butter}

$02 \square$ Oil-butter spreads, Kærgården, Bakkedal, Marklyst, Mælkebøtte, Lurpak butter bar $03 \square$ Frying or baking margarine $70-80 \%$ fat

$04 \square$ Vegetable margarine $60-80 \%$ fat

$05 \square$ Fluid margarine, oil-margarine (eg 'Becel', 'Lise')

$06 \square$ Oil (eg. rapeseed oil, olive oil, corn oil, sunflower oil, grapeseed oil, salad oil etc)

$07 \square$ Use a mixture of oil and butter/Kærgården

$08 \square$ Fat (pig, duck)

$09 \square$ Do not use spread/grease/fat for cooking

$10 \square$ We do not cook/prepare food in our household

$11 \square$ Kasvisterolimargariinia (esim. Becel ProActivem Benecol (only Finland)

$98 \square$ Do not know 
10 How many slices of bread does your child eat per day or per week?

Answer according to slice/piece/1/2 roll

Grain bread also includes wholemeal bread, full grain bread and might carry the wholegrain label. Rolls are also considered bread.

Slice $/$ piece $/ 1 / 2$ roll

Rye Bread

per day per week

Q10.1

How many slices of rye bread does your child eat?

Never eat 97

Do not know 98

10.1a per day

$10.1 \mathrm{~b}$ per week

White Bread or Wholegrain (not rye bread):

Q10.2

How many slices of whole grain bread, with grain does your child eat?

Never eat 97

Do not know 98

10.2a per day

$10.2 \mathrm{~b}$ per week

Q10.3

How many slices of white bread, toastbread, ciabbata does your child eat?

Never eat 97

Do not know 98

10.3a per day

10.3b per week

Q10.4

How many slices of hard bread does your child eat?

Never eat 97

Do not know 98

10.4a per day

$10.4 \mathrm{~b}$ per week 
11 How often does your child eat fruit and vegetables during a day, a week or a month. If your child does not eat fruit and vegetables every day, please think about how often your child eats it in a week or in a month. Think about the last 12 months when you respond.

Please respond to both sub questions, but only one response (cross, $X$ ) on each row

Do not count small portions, eg. a slice of cucumber on bread, parsley as decoration, berries on cake etc.

\begin{tabular}{|c|c|c|c|c|c|c|c|c|c|c|c|c|c|c|c|c|}
\hline & \multicolumn{4}{|c|}{$\begin{array}{c}11.1 \mathrm{a} \\
\text { Times per month }\end{array}$} & \multicolumn{6}{|c|}{$\begin{array}{c}11.1 \mathrm{~b} \\
\text { Times per week }\end{array}$} & \multicolumn{6}{|c|}{$\begin{array}{c}11.1 \mathrm{c} \\
\text { Times per day }\end{array}$} \\
\hline & $<1$ & 1 & 2 & 3 & 1 & $<1$ & 1 & 2 & 3 & 1 & 1 & 2 & 3 & 4 & 5 & $\begin{array}{l}6 \text { or } \\
\text { more }\end{array}$ \\
\hline $\begin{array}{l}\text { How often does } \\
\text { your child eat } \\
\text { vegetables, pulses } \\
\text { and/or root fruits } \\
\text { (includes fresh, } \\
\text { frozen, canned, } \\
\text { glass/potted etc) } \\
\text { DO NOT cOUNT } \\
\text { POTATOES } \\
\text { It is vegetables } \\
\text { such as carrots, } \\
\text { tomatoes, } \\
\text { cucumber, broccoli, } \\
\text { peppers, salad, } \\
\text { beans, chick peas, } \\
\text { lentils, beetroot, } \\
\text { celery and parsnip. } \\
\text { Try also to include } \\
\text { dishes that have } \\
\text { vegetables in them, } \\
\text { such as mixed } \\
\text { salad, mixed } \\
\text { vegetables, fried } \\
\text { vegetables, } \\
\text { vegetable soup and } \\
\text { stews. }\end{array}$ & & $\begin{array}{l}1 \\
\square\end{array}$ & $\begin{array}{l}2 \\
\square\end{array}$ & $\begin{array}{l}3 \\
\square\end{array}$ & $\begin{array}{l}1 \\
\square\end{array}$ & $\begin{array}{l}2 \\
\square\end{array}$ & $\begin{array}{l}3 \\
\square\end{array}$ & $\begin{array}{l}4 \\
\square\end{array}$ & $\begin{array}{l}5 \\
\square\end{array}$ & $\begin{array}{l}6 \\
\square\end{array}$ & & $\begin{array}{l}2 \\
\square\end{array}$ & $\begin{array}{l}3 \\
\square\end{array}$ & $\begin{array}{l}4 \\
\square\end{array}$ & $\begin{array}{l}5 \\
\square\end{array}$ & $\begin{array}{l}6 \\
\square\end{array}$ \\
\hline & & & & & & & & & & & & & & & & \\
\hline
\end{tabular}


11 How often does your child eat fruit and vegetables during a day, a week or a month. If your child does not eat fruit and vegetables every day, please think about how often your child eats it in a week or in a month. Think about the last 12 months when you respond.

Please respond to both sub questions, but only one response (cross, $X$ ) on each row

Do not count small portions, eg. a slice of cucumber on bread, parsley as decoration, berries on cake etc.

\begin{tabular}{|c|c|c|c|c|c|c|c|c|c|c|c|c|c|c|c|c|c|}
\hline & & Tim & $s p$ & mo & & & & es & er w & & & & me & pe & day & & \\
\hline & & $<1$ & 1 & 2 & 3 & 1 & 2 & 3 & 4 & 5 & 6 & 1 & 2 & 3 & 4 & 5 & 6 or \\
\hline 11.2 & $\begin{array}{l}\text { How often does } \\
\text { your child eat fruit } \\
\text { and berries } \\
\text { (includes fresh, } \\
\text { frozen, canned, } \\
\text { glassed/potted etc.) } \\
\text {. Fruit and berries } \\
\text { include: an apple, } \\
\text { an orange, a } \\
\text { banana, a bunch of } \\
\text { grapes, a plate of } \\
\text { strawberries or fruit } \\
\text { and berries that are } \\
\text { part of porridge, } \\
\text { fruit stew, or fruit } \\
\text { salad etc. }\end{array}$ & $\begin{array}{l}0 \\
\square\end{array}$ & $\begin{array}{l}1 \\
\square\end{array}$ & $\begin{array}{l}2 \\
\square\end{array}$ & $\begin{array}{l}3 \\
\square\end{array}$ & $\begin{array}{l}1 \\
\square\end{array}$ & $\begin{array}{l}2 \\
\square\end{array}$ & $\begin{array}{l}3 \\
\square\end{array}$ & $\begin{array}{l}4 \\
\square\end{array}$ & $\begin{array}{l}5 \\
\square\end{array}$ & $\begin{array}{l}6 \\
\square\end{array}$ & & $\begin{array}{l}2 \\
\square\end{array}$ & $\begin{array}{l}3 \\
\square\end{array}$ & $\begin{array}{l}4 \\
\square\end{array}$ & $\begin{array}{l}5 \\
\square\end{array}$ & $\begin{array}{l}6 \\
\square\end{array}$ \\
\hline & & & & & & & & & & & & & & & & & \\
\hline
\end{tabular}




\begin{tabular}{|c|c|c|c|c|c|c|c|c|c|c|c|c|c|c|c|}
\hline & \multicolumn{15}{|c|}{$\begin{array}{l}\text { Now I will ask you how often your child eats and drinks a sele } \\
\text { consider the past } 12 \text { months when you answer these questior } \\
\text { Please answer all questions, but only cross one box on each row }\end{array}$} \\
\hline & & \multicolumn{4}{|c|}{$\begin{array}{c}\mathrm{A} \\
\text { Times per month }\end{array}$} & \multicolumn{6}{|c|}{$\begin{array}{c}\text { B } \\
\text { Times per week }\end{array}$} & \multicolumn{4}{|c|}{\begin{tabular}{|c|} 
C \\
Times per day
\end{tabular}} \\
\hline & & $<1^{*}$ & 1 & 2 & 3 & 1 & 2 & 3 & 4 & 5 & 6 & 1 & 2 & 3 & $\begin{array}{l}4 \text { or } \\
\text { more }\end{array}$ \\
\hline & $\begin{array}{l}\text { How often does } \\
\text { your child } \\
\text { eat/drink? }\end{array}$ & 0 & 1 & 2 & 3 & 1 & 2 & 3 & 4 & 5 & 6 & 1 & 2 & 3 & 4 \\
\hline 12.01 & $\begin{array}{l}\text { French fries, } \\
\text { roasted/fried } \\
\text { potatoes }\end{array}$ & $\square$ & $\square$ & $\square$ & $\square$ & $\square$ & $\square$ & $\square$ & $\square$ & $\square$ & $\square$ & $\square$ & $\square$ & $\square$ & $\square$ \\
\hline 12.02 & $\begin{array}{l}\text { Fish and shellfish } \\
\text { as main course }\end{array}$ & $\square$ & $\square$ & $\square$ & $\square$ & $\square$ & $\square$ & $\square$ & $\square$ & $\square$ & $\square$ & $\square$ & $\square$ & $\square$ & $\square$ \\
\hline 12.03 & $\begin{array}{l}\text { Sausage as a main } \\
\text { course }\end{array}$ & $\square$ & $\square$ & $\square$ & $\square$ & $\square$ & $\square$ & $\square$ & $\square$ & $\square$ & $\square$ & $\square$ & $\square$ & $\square$ & $\square$ \\
\hline 12.04 & $\begin{array}{l}\text { Chocolate and/or } \\
\text { candy }\end{array}$ & $\square$ & $\square$ & $\square$ & $\square$ & $\square$ & $\square$ & $\square$ & $\square$ & $\square$ & $\square$ & $\square$ & $\square$ & $\square$ & $\square$ \\
\hline 12.05 & $\begin{array}{l}\text { Cake, biscuits, tart } \\
\text { etc. }\end{array}$ & $\square$ & $\square$ & $\square$ & $\square$ & $\square$ & $\square$ & $\square$ & $\square$ & $\square$ & $\square$ & $\square$ & $\square$ & $\square$ & $\square$ \\
\hline 12.06 & $\begin{array}{l}\text { Full fat cheese ( } 45- \\
60+\text { or } 24-44 \% \text { fat) }\end{array}$ & $\square$ & $\square$ & $\square$ & $\square$ & $\square$ & $\square$ & $\square$ & $\square$ & $\square$ & $\square$ & $\square$ & $\square$ & $\square$ & $\square$ \\
\hline 12.07 & $\begin{array}{l}\text { Low fat/sugar-free } \\
\text { fizzy drinks, } \\
\text { cordial, ice-tea, } \\
\text { light or sugar free } \\
\text { drinks }\end{array}$ & $\square$ & $\square$ & $\square$ & $\square$ & $\square$ & $\square$ & $\square$ & $\square$ & $\square$ & $\square$ & $\square$ & $\square$ & $\square$ & $\square$ \\
\hline 12.08 & $\begin{array}{l}\text { Normal fizzy drinks, } \\
\text { cordial, ice-tea }\end{array}$ & $\square$ & $\square$ & $\square$ & $\square$ & $\square$ & $\square$ & $\square$ & $\square$ & $\square$ & $\square$ & $\square$ & $\square$ & $\square$ & $\square$ \\
\hline 12.09 & $\begin{array}{l}\text { Energy drinks, red } \\
\text { bull, cult etc. }\end{array}$ & $\square$ & $\square$ & $\square$ & $\square$ & $\square$ & $\square$ & $\square$ & $\square$ & $\square$ & $\square$ & $\square$ & $\square$ & $\square$ & $\square$ \\
\hline 12.10 & $\begin{array}{l}\text { Fruit juice or } \\
\text { Vegetable Juice }\end{array}$ & $\square$ & $\square$ & $\square$ & $\square$ & $\square$ & $\square$ & $\square$ & $\square$ & $\square$ & $\square$ & $\square$ & $\square$ & $\square$ & $\square$ \\
\hline & & & & & & & & & & & & & & & \\
\hline
\end{tabular}


The next questions regard physical activity of the child

[Comment: The interviewer reads three examples of activity. Additional examples are in italics and if the respondents have difficulties in answering, the interviewer can add some of these additional examples on request. Generally the interviewer does not read the text in italics but can use the text as a reminder or can read it on request.]

I am going to ask you about physical activity of your child outside school hours during the last 7 days. Your information is important even if your child has not been physically active.

[Question 13]

I am going to ask you about all physical activity during your child's leisure time and active transportation [e.g. transportation to and from school]. Include all activity where the physical effort is moderate or harder that is, you should include both moderate and vigorous activity. This kind of activity accelerates heart rate and breathing. Examples are bicycle riding, football (soccer) or outdoor games. Additional examples are playing handball, skateboarding; these examples can be age and country specific

During the last 7 days, how much time in total did your child spend in physical activity where the physical effort was moderate or harder? Estimate to the nearest half hour.

The interviewer can help the respondent to narrow down the answer to the nearest half hour. It is important to know if physical activity is less or more than $3 \frac{1}{2}$ hours and if it is more orless than 7 hours

Hours.

Minutes

DK (Dont Know)

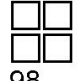

[Question 14a (Sedentary time, TV watching)]

During the last 7 days, how much time per day on average did your child spend sitting and watching TV in his/her leisure time? Estimate it to the nearest half hour. Include videos, DVD and console games (PlayStation, Xbox, etc) played on TV screen.

The interviewer can help the respondent to narrow down the answer to the nearest half hour. It is important to know if average time is less or more than 1 hour and if it is more or less than $2 \frac{1}{2}$ hours

Hours.

Minutes

DK (Dont Know)

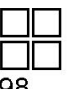


[Question 14b (Sedentary time; Computer screen time)]

Interviewer: During the last 7 days, how much time per day on average did your child spend in front of a computer screen during his/her leisure-time? Estimate to the nearest half an hour. Include video-games, mobile phone games and internet use, and TV programs watched on a computer screen; include home work.

The interviewer can help the respondent to narrow down the answer to the nearest half hour. It is important to know if average time is less or more than 1 hour and if it is more orless than $2 \frac{1}{2}$ hours

Hours

Minutes.

DK (Dont Know)

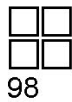

[Question 15 (classification of leisure-time physical activity)]

Choose one of the following descriptions that best fits your child's leisure time activity during the last 7 days.

(1) Reading, watching TV or other sedentary activity.

(2) Walking, cycling, or other forms of light exercise that lasted for at least 4 hours during the last 7 days. Include walking or cycling to and from school, active recreation, etc. [such as skateboarding or rollerb/ading]

(3) Participation in recreational sports [such as football or swimming], active games, etc. [such as tag], where the duration of activity lasted for at least $\mathbf{4}$ hours in total during the last 7 days.

(4) Participation in hard training or sports competitions, regularly several times during the last 7 days. 


\title{
Appendix B: Guidelines for processing
}

\author{
Guidelines for processing data from the Nordic Food Frequency \\ Questionnaire (NFFQ)
}

Data on diet is processed slightly different compared to previous published results on diet (Rasmussen et al. 2012) because missing values are treated differently in this report. Results on "never eat" and "irrelevant" were not both set to missing. The use of weighted data also introduces minor changes regarding data from 2011 compared to previous published results.

Adults and children: Data on bread intake $>15$ slices/day were judged unrealistic and changed to missing. Frequency intake $<1$ time/month was coded as 0.5 time/month, and highest frequency intake $\geq 4$ or more times/day or $\geq 6$ or more times/day was coded as 4 or 6 times/day, respectively. The conversion factor between month and day was 30, and between month and week 4.29. Higher values than possible in a categori was not judged as unrealistic/missing, but set to highest frequency category.

\section{Guidelines for processing and categorizing data from the Nordic Physical Activity Questionnaire (NPAQ)}

Adults and children: Data that were not rounded to the nearest $1 / 2$ hour by the interviewer were rounded to the nearest $1 / 2$ hour and hours and minutes were added together before data processing and further calculations were done in hours. Only participants who reported valid data for moderate to vigorous physical activity (MVPA), vigorous physical activity (VPA; adults), and/or TV time and computer time were included in the analyses.

MVPA adults and children: Data on MVPA $>21$ hours/week and $\leq 35$ hours/week were truncated down to 21 hours/week. Data on MVPA > 35 hours/week were judged unrealistic and set to missing. 
VPA adults: Data on VPA $>10$ hours/week and $\leq 21$ hours/week were truncated down to 10 hours/week. Data on VPA $>21$ hours/week or VPA-hours $>$ MVPA-hours were judged unrealistic and set to missing.

MPA adults: Moderate physical activity (MPA) was calculated by subtracting VPA from MVPA (MPA=MVPA-VPA). Calculation of MPA should be done in this step (after rounding to the nearest hour, adding hours and minutes together and truncating down high values) in order to get valid data.

Screen time (TV + computer time) adults and children: Data on screen time (TV + computer time) $>12$ hours/day and $\leq 18$ hours/day were truncated down to 12 hours/day using weighted truncation. Data on screen time (TV + computer time) $>18$ hours/day were judged unrealistic and set to missing (TV and computer time).

\section{Calculation of activity categories}

Compliance with physical activity (PA) recommendations adults: To determine compliance with the recommendations on physical activity among adults, six different physical activity categories (PAC) were calculated from MPA and VPA. Then a cut-point (cp) ratio was calculated for both MPA and VPA:

- $\quad M^{\prime} A_{\text {ratio }}=M P A / M P A_{c p} . M P A_{c p}$ (cut-point for MPA) was set at 3,5 hours/week.

- VPA ratio=VPA/VPA $_{c p}$. VPA $A_{c p}$ (cut-point for VPA) was set to 1,25 hours/week.

Six PAC groups were calculated:

- $P A C=0: M P A_{\text {ratio }}=0$ and $V P A_{\text {ratio }}=0$ (highly inactive - not meeting $P A$ recommendations).

- $P A C=1: M P A_{\text {ratio }}+V P A_{\text {ratio }}<1.0$ (inactive - not meeting $P A$ recommendations).

- $P A C=2: M^{\prime} A_{r a t i o} \geq 1.0$ and $V P A_{\text {ratio }}<1.0$ (active - meeting PA recommendations by moderate intensity $P A)$.

- $\quad P A C=3: M A_{\text {ratio }}<1.0$ and $V P A_{\text {ratio }} \geq 1.0$ (active - meeting PA recommendations by vigorous intensity PA).

- $P A C=4: \mathrm{MPA}_{\text {ratio }}+\mathrm{VPA}_{\text {ratio }} \geq 1.0$ (active - meeting PA recommendations by a combination of moderate and vigorous intensity $P A)$.

- $P A C=5: M P A_{\text {ratio }} \geq 1.0$ and $V P A_{\text {ratio }} \geq 1.0$ (highly active - meeting full PA recommendations, i.e. meeting both moderate and vigorous intensity recommendations).

Compliance with physical activity recommendations children: To determine compliance with the recommendation on physical activity among children, three different physical activity categories were defined as:

- $\quad P A C=0$ : Highly inactive (not meeting PA recommendation): $<0,25$ MVPA hours/week.

- $\quad \mathrm{PAC}=1$ : Inactive (not meeting PA recommendation): $\geq 0,25 \mathrm{MVPA}$ hours/week and $<7 \mathrm{MVPA}$ hours/week.

- $\quad \mathrm{PAC}=2$ : Active (meeting PA recommendation): $\geq 7,0 \mathrm{MVPA}$ hours/week. 


\section{Appendix C: Goals 2011 and Visions 2021 in the Nordic Plan of Action}

Tabel 96: How to evaluate goals and visions

\begin{tabular}{|c|c|c|c|c|}
\hline & Goal 2011 & $\begin{array}{l}\text { How to evaluate goal } \\
\text { 2011? }\end{array}$ & Vision 2021 & How to evaluate vision $2021 ?$ \\
\hline \multirow[t]{4}{*}{ Diet } & $\begin{array}{l}\text { "The consump- } \\
\text { tion of fruits } \\
\text { and vegetables } \\
\text { has increased." }\end{array}$ & $\begin{array}{l}\text { Examine the develop- } \\
\text { ment of fruit and vege- } \\
\text { tables (excl. fruit juice) } \\
\text { intake among adults and } \\
\text { children. } \\
\text { NORMO } 2011-2014\end{array}$ & $\begin{array}{l}\text { "At least } 70 \% \text { of the popu- } \\
\text { lation above } 10 \text { years has } \\
\text { a daily intake of fruits and } \\
\text { vegetables of at least } 500 \\
\text { g/day." }\end{array}$ & $\begin{array}{l}\text { Examine the population levels of } \\
\text { " } 5+\text { a day" of fruit and vegetables } \\
\text { (incl. fruit juice) among adults and } \\
\text { children. }{ }^{22} \\
\text { NORMO } 2011-2014\end{array}$ \\
\hline & $\begin{array}{l}\text { "The consump- } \\
\text { tion of fish has } \\
\text { increased." } 23\end{array}$ & $\begin{array}{l}\text { Examine the develop- } \\
\text { ment of fish intakea- } \\
\text { mong adults and chil- } \\
\text { dren. } \\
\text { NORMO 2011-2014 }\end{array}$ & $\begin{array}{l}\text { "70\% or more consumes } \\
\text { fish or fish products, cor- } \\
\text { responding to a main dish } \\
\text { twice a week." }\end{array}$ & $\begin{array}{l}\text { Examine the population levels of } \\
\text { fish intake (fish as a main course } \\
\text { twice a week) among adults and } \\
\text { children. } \\
\text { NORMO } 2011-2014\end{array}$ \\
\hline & $\begin{array}{l}\text { "The consump- } \\
\text { tion of whole- } \\
\text { grain bread/ } \\
\text { cereals has in- } \\
\text { creased." }\end{array}$ & $\begin{array}{l}\text { Examine the develop- } \\
\text { ment of wholegrain/cere- } \\
\text { als intakeamong adults } \\
\text { and children. } \\
\text { NORMO 2011-2014 }\end{array}$ & $\begin{array}{l}\text { "At least } 70 \% \text { of the adult } \\
\text { population has a daily in- } \\
\text { take of whole-grain } \\
\text { bread/cereals correspond- } \\
\text { ing to at least half of their } \\
\text { daily intake of bread/cere- } \\
\text { als." }\end{array}$ & $\begin{array}{l}\text { Examine the population levels of } \\
\text { whole grain intake (daily bread in- } \\
\text { take where at least half of all } \\
\text { bread eaten is whole grain) among } \\
\text { adults and children. } \\
\text { NORMO } 2011-2014\end{array}$ \\
\hline & $\begin{array}{l}\text { "The intake of } \\
\text { fat, especially } \\
\text { saturated fat } \\
\text { and trans fatty } \\
\text { acids, has been } \\
\text { reduced." }\end{array}$ & $\begin{array}{l}\text { Examine the development } \\
\text { of saturated fat intake } \\
\text { among adults and children } \\
\text { using a fat index }{ }^{24} \text { based } \\
\text { on a high intake of full fat } \\
\text { cheese intake, and type of } \\
\text { fat used on bread and for } \\
\text { cooking). } \\
\text { NORMO 2011-2014 }\end{array}$ & $\begin{array}{l}\text { "The average dietary intake } \\
\text { of the population meets the } \\
\text { NNR on saturated fat plus } \\
\text { trans fatty acids (less than } \\
10 \text { E\% from saturated fat), } \\
\text { and at least } 70 \% \text { meets the } \\
\text { NNR on fat (E\% from fat be- } \\
\text { tween } 25 \text { and } 40) . " 25\end{array}$ & $\begin{array}{l}\text { Examine the population levels and } \\
\text { the development meeting the NNR } \\
\text { recommendation on total fat and } \\
\text { saturated fat among adults and } \\
\text { children using other national esti- } \\
\text { mates from the Nordic countries. } \\
\text { Other national estimates from the } \\
\text { Nordic countries }\end{array}$ \\
\hline
\end{tabular}

\footnotetext{
22 Intake of fruit juice is adjusted, so that intake frequencies of juice $>1 /$ day are calculated as $1 /$ day.

23 Fish is not mentioned in Goal 2011 in the Nordic Plan of Action (Nordic Council of Ministers 2006), but a goal of an increased intake of fish is suggested by the NORMO project group.

${ }_{24} \mathrm{High}$ saturated fat index group, i.e. high frequency intake of full fat cheese and a high content of saturated fat used on bread and for cooking.

25 The upper limit for saturated fat intake has changed from $\max 10 \mathrm{E} \%$ to less than $10 \mathrm{E} \%$. In addition, the upper limit for the total fat intake has changed from $35 \mathrm{E} \%$ to $40 \%$ and the population goal on $30 \mathrm{E} \%$ from fat is in no longer used in the Nordic Nutrition Recommendations (Nordic Nutrition Recommendations 2012).
} 


\begin{tabular}{|c|c|c|c|c|}
\hline & Goal 2011 & $\begin{array}{l}\text { How to evaluate goal } \\
\text { 2011? }\end{array}$ & Vision 2021 & How to evaluate vision $2021 ?$ \\
\hline \multicolumn{5}{|c|}{ Table 96 continued } \\
\hline & $\begin{array}{l}\text { "The intake of } \\
\text { added sugar has } \\
\text { been reduced." }\end{array}$ & $\begin{array}{l}\text { Examine the develop- } \\
\text { ment of added sugar in- } \\
\text { take among adults and } \\
\text { children using a sugar in- } \\
\text { dex based on a high in- } \\
\text { take of chocolate/candy, } \\
\text { cake and soft drinks. }{ }^{26} \\
\text { NORMO } 2011-2014\end{array}$ & $\begin{array}{l}\text { "80\% or more meets the } \\
\text { NNR recommendation on } \\
\text { daily intake of added sugar } \\
\text { (max. } 10 \text { E\%)." }\end{array}$ & $\begin{array}{l}\text { Examine the population levels } \\
\text { and the development meeting } \\
\text { the NNR recommendation on } \\
\text { added sugar among adults and } \\
\text { children using other national esti- } \\
\text { mates from the Nordic countries. } \\
\text { Other national estimates from the } \\
\text { Nordic countries }\end{array}$ \\
\hline \multirow[t]{2}{*}{$\begin{array}{l}\text { Physical } \\
\text { activity }\end{array}$} & $\begin{array}{l}\text { "The current } \\
\text { trend, where an } \\
\text { increasing pro- } \\
\text { portion of } \\
\text { adults are physi- } \\
\text { cally inactive, }{ }^{27} \\
\text { has been } \\
\text { brought to a } \\
\text { halt and at best } \\
\text { reversed." }\end{array}$ & $\begin{array}{l}\text { Examine the develop- } \\
\text { ment of physical activity } \\
\text { (inactive) and sedentary } \\
\text { behaviour (high recrea- } \\
\text { tional screen time) } \\
\text { among adults. }{ }^{28} \\
\text { NORMO 2011-2014 }\end{array}$ & $\begin{array}{l}\text { "At least } 75 \% \text { of the adult } \\
\text { population is physically ac- } \\
\text { tive (moderate intensity) } \\
\text { for at least } 30 \text { minutes } \\
\text { every day." }\end{array}$ & $\begin{array}{l}\text { Examine the population levels } \\
\text { meeting the physical activity rec- } \\
\text { ommendations among adults. } \\
\text { NORMO 2011-2014 }\end{array}$ \\
\hline & $\begin{array}{l}\text { "The current } \\
\text { trend, where an } \\
\text { increasing pro- } \\
\text { portion of chil- } \\
\text { dren are physi- } \\
\text { cally inactive, }{ }^{3} \\
\text { has been } \\
\text { brought to a } \\
\text { halt and at best } \\
\text { reversed." }\end{array}$ & $\begin{array}{l}\text { Examine the develop- } \\
\text { ment of physical activity } \\
\text { (inactive) and sedentary } \\
\text { behaviour (high recrea- } \\
\text { tional screen time). } \\
\text { NORMO 2011-2014 }\end{array}$ & $\begin{array}{l}\text { "All children aged 5-12 are } \\
\text { physically active (moder- } \\
\text { ate intensity) for at least } 1 \\
\text { hour every day." } 29\end{array}$ & $\begin{array}{l}\text { Examine the population levels } \\
\text { meeting the physical activity rec- } \\
\text { ommendations among children. } \\
\text { NORMO 2011-2014 }\end{array}$ \\
\hline $\begin{array}{l}\text { Over- } \\
\text { weight/ } \\
\text { obesity }\end{array}$ & $\begin{array}{l}\text { "The continuing } \\
\text { increase in the } \\
\text { proportion of } \\
\text { the overweight } \\
\text { and obese } \\
\text { adults } \\
\text { has been } \\
\text { stopped and at } \\
\text { best reversed." }\end{array}$ & $\begin{array}{l}\text { Examine the develop- } \\
\text { ment of overweight and } \\
\text { obesity among adults. } \\
\text { NORMO 2011-2014 }\end{array}$ & $\begin{array}{l}\text { "The number of over- } \\
\text { weight and obese adults } \\
\text { has been reduced by at } \\
\text { least } 30 \% \text { from the pre- } \\
\text { sent level." } 30\end{array}$ & $\begin{array}{l}\text { Examine the prevalence and the } \\
\text { development of overweight and } \\
\text { obesity among adults using } \\
\text { NORMO data and other national } \\
\text { estimates from the Nordic coun- } \\
\text { tries. } \\
\text { NORMO } 2014 \text { and other national } \\
\text { estimates from the Nordic coun- } \\
\text { tries }\end{array}$ \\
\hline
\end{tabular}

\footnotetext{
${ }^{26} \mathrm{High}$ sugar index group, i.e. high frequency intake of sugar-rich foods (chocolate/candy, cake and soft drinks).

${ }_{27}$ Physically inactive is insufficient active and is defined as a failure to meet the physical activity recommendations (Nordic Nutrition Recommendations 2012)

${ }^{28} \mathrm{High}$ recreational screen time ( $>4$ hours/day of TV and computer time) was used as an indicator of sedentary behaviour. 29 The physical activity recommendations stating that children and young people should accumulate at least one hour of moderate to vigorous intensity physical activity daily is targeted to 5-17-y-olds ( $W H O 2010$ ).

$3^{30}$ The present level refers to year 2006 where the Nordic Plan of Action was launched.
} 


\begin{tabular}{|c|c|c|c|c|}
\hline & Goal 2011 & $\begin{array}{l}\text { How to evaluate goal } \\
\text { 2011? }\end{array}$ & Vision 2021 & How to evaluate vision $2021 ?$ \\
\hline \multicolumn{5}{|c|}{ Table 96 continued } \\
\hline & $\begin{array}{l}\text { "The continuing } \\
\text { increase in the } \\
\text { proportion of } \\
\text { the overweight } \\
\text { and obese chil- } \\
\text { dren }\end{array}$ & $\begin{array}{l}\text { Examine the develop- } \\
\text { ment of overweight and } \\
\text { obesity among children. } \\
\text { NORMO 2011-2014 }\end{array}$ & $\begin{array}{l}\text { "The number of over- } \\
\text { weight and obese children } \\
\text { has been reduced by at } \\
\text { least } 50 \% \text { from the pre- } \\
\text { sent level." } 34\end{array}$ & $\begin{array}{l}\text { Examine the prevalence and the } \\
\text { development of overweight and } \\
\text { obesity among children using } \\
\text { NORMO data and other national } \\
\text { estimates from the Nordic coun- } \\
\text { tries. }\end{array}$ \\
\hline & $\begin{array}{l}\text { has been } \\
\text { stopped and at } \\
\text { best reversed." }\end{array}$ & & & $\begin{array}{l}\text { NORMO } 2014 \text { and other national } \\
\text { estimates from the Nordic coun- } \\
\text { tries }\end{array}$ \\
\hline $\begin{array}{l}\text { Social ine- } \\
\text { quality in } \\
\text { over- } \\
\text { weight/obe } \\
\text { sity and } \\
\text { diet and } \\
\text { physical } \\
\text { activity }\end{array}$ & $\begin{array}{l}\text { "Existing differ- } \\
\text { ences between } \\
\text { different social } \\
\text { groups with re- } \\
\text { gard to over- } \\
\text { weight, obesity, } \\
\text { unhealthy diet, } \\
\text { and physical in- } \\
\text { activity have } \\
\text { not deepened } \\
\text { further and at } \\
\text { best have been } \\
\text { reduced." }\end{array}$ & $\begin{array}{l}\text { Examine the develop- } \\
\text { ment of diet (unhealthy } \\
\text { diet), physical activity } \\
\text { (inactive), sedentary be- } \\
\text { havior (high recreational } \\
\text { screen time), and over- } \\
\text { weight and obesity } \\
\text { among adults and chil- } \\
\text { dren in the low and high } \\
\text { education group. }{ }^{31} \\
\text { NORMO 2011-2014 }\end{array}$ & $\begin{array}{l}\text { "The difference between } \\
\text { different social groups on } \\
\text { meeting the defined ob- } \\
\text { jectives with regard to } \\
\text { diet, physical activity, and } \\
\text { overweight/obesity has } \\
\text { decreased and is at most } \\
20 \% \text { between groups." }\end{array}$ & $\begin{array}{l}\text { Examine the population levels } \\
\text { and the development of diet (un- } \\
\text { healthy diet), physical activity (in- } \\
\text { active) and overweight among } \\
\text { adults and children in the low and } \\
\text { high education group. Examine if } \\
\text { the relative difference between } \\
\text { the low and high education group } \\
\text { exceeds } 20 \% \text { in } 2014 \text {. } \\
\text { NORMO } 2011-2014\end{array}$ \\
\hline
\end{tabular}

\section{References}

Nordic Nutrition Recommendations 2012. Integrating nutrition and physical activity. 5th ed. Copenhagen: Nordic Council of Ministers; 2014.

Nordic Council of Ministers. Health, food and physical activity. Nordic Plan of Action on better health and quality of life through diet and physical activity, Copenhagen 2006.

WHO. Global recommendations on physical activity for health. World Health Organization 2010.

${ }^{31}$ Low education is defined as basic education ( $<10 \mathrm{y}$ ), and high education is defined as low, medium or long higher education ( $\geq$ $13 \mathrm{y}$ ). Medium education is defined as vocational or upper secondary education (10-12 y). 

Appendix D: Additional tables 
Foods

Table 97: Mean $(95 \% \mathrm{Cl})$ intake of selected foods among adults in the Nordic countries. NORMO 2011 and 2014

\begin{tabular}{|c|c|c|c|c|c|c|c|c|c|c|c|c|}
\hline & \multicolumn{2}{|c|}{ Denmark } & \multicolumn{2}{|c|}{ Finland } & \multicolumn{2}{|c|}{ Sweden } & \multicolumn{2}{|c|}{ Iceland } & \multicolumn{2}{|c|}{ Norway } & \multicolumn{2}{|c|}{ Nordic region $₫$} \\
\hline & $\begin{array}{r}2011 \\
(n=1,943)\end{array}$ & $\begin{array}{r}2014 \\
(n=1,969)\end{array}$ & $\begin{array}{r}2011 \\
(n=1,966)\end{array}$ & $\begin{array}{r}2014 \\
(n=1,561)\end{array}$ & $\begin{array}{r}2011 \\
(n=1,834)\end{array}$ & $\begin{array}{r}2014 \\
(n=1,700)\end{array}$ & $\begin{array}{r}2011 \\
(n=1,946)\end{array}$ & $\begin{array}{r}2014 \\
(n=1,916)\end{array}$ & $\begin{array}{r}2011 \\
(n=1,035)\end{array}$ & $\begin{array}{r}2014 \\
(n=961)\end{array}$ & $\begin{array}{r}2011 \\
(n=8,833)\end{array}$ & $\begin{array}{r}2014 \\
(n=8,144)\end{array}$ \\
\hline $\begin{array}{l}\text { Rye bread } \\
\text { (slices/day) }\end{array}$ & $\begin{array}{r}2.4 \\
(2.3 ; 2.4)\end{array}$ & $\begin{array}{r}1.8^{* * * *} \\
(1.8 ; 1.9)\end{array}$ & $\begin{array}{r}2.6 \\
(2.5 ; 2.7)\end{array}$ & $\begin{array}{r}2.3 * * * \\
(2.2 ; 2.4)\end{array}$ & - & - & $\begin{array}{r}0.7 \\
(0.7 ; 0.8)\end{array}$ & $\begin{array}{r}0.7 \\
(0.7 ; 0.7)\end{array}$ & $\begin{array}{r}0.9 \\
(0.8 ; 1.1)\end{array}$ & $\begin{array}{r}0.8^{-} \\
(0.7 ; 0.9)\end{array}$ & $\begin{array}{r}1.3 \\
(1.2 ; 1.3)\end{array}$ & $\begin{array}{r}1.1 * * * \\
(1.0 ; 1.1)\end{array}$ \\
\hline $\begin{array}{l}\text { Wholemeal bread } \\
\text { (slices/day) }\end{array}$ & $\begin{array}{r}1.1 \\
(1.1 ; 1.2)\end{array}$ & $\begin{array}{r}1.1 \\
(1.1 ; 1.2)\end{array}$ & $\begin{array}{r}1.1 \\
(1.1 ; 1.2)\end{array}$ & $\begin{array}{r}1.0^{*} \\
(0.9 ; 1.1)\end{array}$ & $\begin{array}{r}1.6 \\
(1.6 ; 1.7)\end{array}$ & $\begin{array}{l}1.4^{* * *} \\
(1.3 ; 1.5)\end{array}$ & $\begin{array}{r}1.0 \\
(1.0 ; 1.1)\end{array}$ & $\begin{array}{r}0.7^{* * *} \\
(0.7 ; 0.8)\end{array}$ & $\begin{array}{r}3.0 \\
(2.8 ; 3.2)\end{array}$ & $\begin{array}{r}2.5^{* * *} \\
(2.4 ; 2.7)\end{array}$ & $\begin{array}{r}1.7 \\
(1.6 ; 1.7)\end{array}$ & $\begin{array}{r}1.5^{* * *} \\
(1.4 ; 1.5)\end{array}$ \\
\hline $\begin{array}{l}\text { White bread } \\
\text { (slices/day) }\end{array}$ & $\begin{array}{r}0.6 \\
(0.5 ; 0.6)\end{array}$ & $\begin{array}{r}0.5^{*} \\
(0.4 ; 0.5)\end{array}$ & $\begin{array}{r}0.4 \\
(0.4 ; 0.5)\end{array}$ & $\begin{array}{r}0.5 \\
(0.4 ; 0.5)\end{array}$ & $\begin{array}{r}0.5 \\
(0.5 ; 0.6)\end{array}$ & $\begin{array}{r}0.5 \\
(0.5 ; 0.6)\end{array}$ & $\begin{array}{r}0.1 \\
(0.1 ; 0.1)\end{array}$ & $\begin{array}{r}0.2 * * * \\
(0.2 ; 0.2)\end{array}$ & $\begin{array}{r}0.2 \\
(0.2 ; 0.3)\end{array}$ & $\begin{array}{r}0.3 \\
(0.2 ; 0.3)\end{array}$ & $\begin{array}{r}0.4 \\
(0.4 ; 0.5)\end{array}$ & $\begin{array}{r}0.4 \\
(0.4 ; 0.5\end{array}$ \\
\hline $\begin{array}{l}\text { Hard bread } \\
\text { (slices/day) }\end{array}$ & $\begin{array}{r}0.3 \\
(0.3 ; 0.4)\end{array}$ & $\begin{array}{r}0.3 \\
(0.3 ; 0.4)\end{array}$ & $\begin{array}{r}0.5 \\
(0.4 ; 0.5)\end{array}$ & $\begin{array}{r}0.4 \\
(0.4 ; 0.5)\end{array}$ & $\begin{array}{r}1.0 \\
(1.0 ; 1.1)\end{array}$ & $\begin{array}{r}1.0 \\
(0.9 ; 1.0)\end{array}$ & $\begin{array}{r}0.7 \\
(0.6 ; 0.7)\end{array}$ & $\begin{array}{r}0.8^{*} \\
(0.7 ; 0.8)\end{array}$ & $\begin{array}{r}1.1 \\
(1.0 ; 1.2)\end{array}$ & $\begin{array}{r}1.2 \\
(1.1 ; 1.3)\end{array}$ & $\begin{array}{r}0.8 \\
(0.7 ; 0.8)\end{array}$ & $\begin{array}{r}0.8 \\
(0.7 ; 0.8)\end{array}$ \\
\hline $\begin{array}{l}\text { Vegetables } \\
\text { (freq./day) }\end{array}$ & $\begin{array}{r}1.1 \\
(1.1 ; 1.1)\end{array}$ & $\begin{array}{l}1.2 * * * \\
(1.2 ; 1.2)\end{array}$ & $\begin{array}{r}1.3 \\
(1.2 ; 1.3)\end{array}$ & $\begin{array}{l}1.4^{* * *} \\
(1.3 ; 1.4)\end{array}$ & $\begin{array}{r}1.3 \\
(1.2 ; 1.3)\end{array}$ & $\begin{array}{l}1.5^{* * *} \\
(1.5 ; 1.5)\end{array}$ & $\begin{array}{r}1.1 \\
(1.0 ; 1.1)\end{array}$ & $\begin{array}{r}1.1 \\
(1.0 ; 1.1)\end{array}$ & $\begin{array}{r}1.7 \\
(1.6 ; 1.7)\end{array}$ & $\begin{array}{l}1.2 * * * \\
(1.1 ; 1.2)\end{array}$ & $\begin{array}{r}1.3 \\
(1.3 ; 1.3)\end{array}$ & $\begin{array}{r}1.3^{*} \\
(1.3 ; 1.4)\end{array}$ \\
\hline $\begin{array}{l}\text { Fruit }{ }^{t} \\
\text { (freq./day) }\end{array}$ & $\begin{array}{r}1.5 \\
(1.4 ; 1.5)\end{array}$ & $\begin{array}{r}1.4^{* *} \\
(1.3 ; 1.4)\end{array}$ & $\begin{array}{r}1.1 \\
(1.0 ; 1.1)\end{array}$ & $\begin{array}{r}1.2 * * \\
(1.1 ; 1.2)\end{array}$ & $\begin{array}{r}1.3 \\
(1.2 ; 1.3)\end{array}$ & $\begin{array}{r}1.2 * * \\
(1.1 ; 1.2)\end{array}$ & $\begin{array}{r}1.1 \\
(1.0 ; 1.1)\end{array}$ & $\begin{array}{r}1.1 \\
(1.0 ; 1.1)\end{array}$ & $\begin{array}{r}1.2 \\
(1.1 ; 1.3)\end{array}$ & $\begin{array}{r}1.1 * \\
(1.1 ; 1.2)\end{array}$ & $\begin{array}{r}1.2 \\
(1.2 ; 1.3)\end{array}$ & $\begin{array}{l}1.2 * * * \\
(1.2 ; 1.2)\end{array}$ \\
\hline $\begin{array}{l}\text { Fruit juice } \\
\text { (freq./day) }\end{array}$ & $\begin{array}{r}0.3 \\
(0.3 ; 0.3)\end{array}$ & $\begin{array}{r}0.3^{* * * *} \\
(0.2 ; 0.3)\end{array}$ & $\begin{array}{r}0.4 \\
(0.4 ; 0.4)\end{array}$ & $\begin{array}{l}0.3^{* * *} \\
(0.3 ; 0.4)\end{array}$ & $\begin{array}{r}0.4 \\
(0.3 ; 0.4)\end{array}$ & $\begin{array}{r}0.3^{* *} \\
(0.3 ; 0.3)\end{array}$ & $\begin{array}{r}0.5 \\
(0.5 ; 0.5)\end{array}$ & $\begin{array}{l}0.4^{* * *} \\
(0.3 ; 0.4)\end{array}$ & $\begin{array}{r}0.5 \\
(0.5 ; 0.6)\end{array}$ & $\begin{array}{l}0.4^{* * *} \\
(0.4 ; 0.4)\end{array}$ & $\begin{array}{r}0.4 \\
(0.4 ; 0.4)\end{array}$ & $\begin{array}{l}0.3^{* * *} \\
(0.3 ; 0.3)\end{array}$ \\
\hline $\begin{array}{l}\text { Fish } \\
\text { (freq./week) }\end{array}$ & $\begin{array}{r}1.3 \\
(1.2 ; 1.3)\end{array}$ & $\begin{array}{r}1.1 * \\
(1.1 ; 1.2)\end{array}$ & $\begin{array}{r}1.3 \\
(1.3 ; 1.4)\end{array}$ & $\begin{array}{r}1.4 \\
(1.3 ; 1.5)\end{array}$ & $\begin{array}{r}1.4 \\
(1.4 ; 1.5)\end{array}$ & $\begin{array}{r}1.5 \\
(1.4 ; 1.5)\end{array}$ & $\begin{array}{r}2.0 \\
(1.9 ; 2.0)\end{array}$ & $\begin{array}{r}2.0 \\
(1.9 ; 2.1)\end{array}$ & $\begin{array}{r}2.2 \\
(2.0 ; 2.3)\end{array}$ & $\begin{array}{r}1.8^{* * * *} \\
(1.7 ; 1.9)\end{array}$ & $\begin{array}{r}1.5 \\
(1.5 ; 1.6)\end{array}$ & $\begin{array}{r}1.5^{* *} \\
(1.4 ; 1.5)\end{array}$ \\
\hline $\begin{array}{l}\text { French fries } \\
\text { (freq./month) }\end{array}$ & $\begin{array}{r}3.2 \\
(2.9 ; 3.5)\end{array}$ & $\begin{array}{r}3.2 \\
(3.0 ; 3.4)\end{array}$ & $\begin{array}{r}2.0 \\
(1.9 ; 2.2)\end{array}$ & $\begin{array}{r}2.1 \\
(2.0 ; 2.3)\end{array}$ & $\begin{array}{r}2.9 \\
(2.7 ; 3.1)\end{array}$ & $\begin{array}{r}3.1 \\
(2.9 ; 3.3)\end{array}$ & $\begin{array}{r}3.1 \\
(2.9 ; 3.3)\end{array}$ & $\begin{array}{r}3.2 \\
(3.0 ; 3.4)\end{array}$ & $\begin{array}{r}2.0 \\
(1.8 ; 2.2)\end{array}$ & $\begin{array}{r}2.5^{* *} \\
(2.2 ; 2.9)\end{array}$ & $\begin{array}{r}2.6 \\
(2.5 ; 2.7)\end{array}$ & $\begin{array}{r}2.8^{* *} \\
(2.7 ; 2.9)\end{array}$ \\
\hline $\begin{array}{l}\text { Sausage } \\
\text { (freq./month) }\end{array}$ & $\begin{array}{r}1.2 \\
(1.0 ; 1.4)\end{array}$ & $\begin{array}{r}1.4 \\
(1.2 ; 1.6)\end{array}$ & $\begin{array}{r}2.5 \\
(2.3 ; 2.7)\end{array}$ & $\begin{array}{r}2.3 \\
(2.1 ; 2.5)\end{array}$ & $\begin{array}{r}3.8 \\
(3.6 ; 4.0)\end{array}$ & $\begin{array}{r}3.5^{* *} \\
(3.333 .6)\end{array}$ & $\begin{array}{r}2.4 \\
(2.3 ; 2.6)\end{array}$ & $\begin{array}{r}2.0^{* * * *} \\
(1.9 ; 2.1)\end{array}$ & $\begin{array}{r}2.1 \\
(1.9 ; 2.3)\end{array}$ & $\begin{array}{r}2.6^{*} \\
(2.2 ; 3.0)\end{array}$ & $\begin{array}{r}2.6 \\
(2.5 ; 2.7)\end{array}$ & $\begin{array}{r}2.6 \\
(2.5 ; 2.7)\end{array}$ \\
\hline $\begin{array}{l}\text { Chocolate and candy } \\
\text { (freq./month) }\end{array}$ & $\begin{array}{r}12.0 \\
(11.4 ; 12.6)\end{array}$ & $\begin{array}{r}12.7 \\
(11.9 ; 13.4)\end{array}$ & $\begin{array}{r}8.9 \\
(8.3 ; 9.4)\end{array}$ & $\begin{array}{r}8.7 \\
(8.2 ; 9.3)\end{array}$ & $\begin{array}{r}8.6 \\
(8.1 ; 9.1)\end{array}$ & $\begin{array}{r}8.3 \\
(7.8 ; 8.8)\end{array}$ & $\begin{array}{r}12.0 \\
(11.5 ; 12.5)\end{array}$ & $\begin{array}{r}12.7^{-} \\
(12.1 ; 13.2)\end{array}$ & $\begin{array}{r}10.6 \\
(9.7 ; 11.5)\end{array}$ & $\begin{array}{r}10.0 \\
(9.1 ; 10.8)\end{array}$ & $\begin{array}{r}9.8 \\
(9.5 ; 10.1)\end{array}$ & $\begin{array}{r}9.7 \\
(9.4 ; 10.0)\end{array}$ \\
\hline $\begin{array}{l}\text { Cake } \\
\text { (freq./month) }\end{array}$ & $\begin{array}{r}7.3 \\
(6.9 ; 7.7)\end{array}$ & $\begin{array}{r}6.4^{* *} \\
(6.0 ; 6.7)\end{array}$ & $\begin{array}{r}9.9 \\
(9.4 ; 10.5)\end{array}$ & $\begin{array}{r}9.0^{*} \\
(8.5 ; 9.7)\end{array}$ & $\begin{array}{r}7.4 \\
(6.8 ; 7.9)\end{array}$ & $\begin{array}{r}6.2 * * \\
(5.8 ; 6.6)\end{array}$ & $\begin{array}{r}9.4 \\
(8.9 ; 9.9)\end{array}$ & $\begin{array}{r}8.1 * * * * \\
(7.7 ; 8.5)\end{array}$ & $\begin{array}{r}5.1 \\
(4.5 ; 5.6)\end{array}$ & $\begin{array}{r}5.1 \\
(4.3 ; 5.9)\end{array}$ & $\begin{array}{r}7.5 \\
(7.2 ; 7.7)\end{array}$ & $\begin{array}{r}6.6 * * * \\
(6.4 ; 6.9)\end{array}$ \\
\hline
\end{tabular}




\begin{tabular}{|c|c|c|c|c|c|c|c|c|c|c|c|c|}
\hline & \multicolumn{2}{|c|}{ Denmark } & \multicolumn{2}{|c|}{ Finland } & \multicolumn{2}{|c|}{ Sweden } & \multicolumn{2}{|c|}{ Iceland } & \multicolumn{2}{|c|}{ Norway } & \multicolumn{2}{|c|}{ Nordic region@ } \\
\hline & $\begin{array}{r}2011 \\
(n=1,943)\end{array}$ & $\begin{array}{r}2014 \\
(n=1,969)\end{array}$ & $\begin{array}{r}2011 \\
(n=1,966)\end{array}$ & $\begin{array}{r}2014 \\
(n=1,561)\end{array}$ & $\begin{array}{r}2011 \\
(n=1,834)\end{array}$ & $\begin{array}{r}2014 \\
(n=1,700)\end{array}$ & $\begin{array}{r}2011 \\
(n=1,946)\end{array}$ & $\begin{array}{r}2014 \\
(n=1,916)\end{array}$ & $\begin{array}{r}2011 \\
(n=1,035)\end{array}$ & $\begin{array}{r}2014 \\
(n=961)\end{array}$ & $\begin{array}{r}2011 \\
(n=8,833)\end{array}$ & $\begin{array}{r}2014 \\
(n=8,144)\end{array}$ \\
\hline Table 97 continued & & & & & & & & & & & & \\
\hline $\begin{array}{l}\text { Cheese } \\
\text { (freq./day) }\end{array}$ & $\begin{array}{r}0.6 \\
(0.5 ; 0.6)\end{array}$ & $\begin{array}{l}0.5^{* * *} \\
(0.5 ; 0.5)\end{array}$ & $\begin{array}{r}0.6 \\
(0.5 ; 0.6)\end{array}$ & $\begin{array}{r}0.6 \\
(0.5 ; 0.6)\end{array}$ & $\begin{array}{r}0.7 \\
(0.7 ; 0.7)\end{array}$ & $\begin{array}{r}0.7^{*} \\
(0.7 ; 0.8)\end{array}$ & $\begin{array}{r}0.5 \\
(0.5 ; 0.5)\end{array}$ & $\begin{array}{r}0.6^{* *} \\
(0.5 ; 0.6)\end{array}$ & $\begin{array}{r}0.9 \\
(0.8 ; 0.9)\end{array}$ & $\begin{array}{r}0.9 \\
(0.8 ; 0.9)\end{array}$ & $\begin{array}{r}0.7 \\
(0.6 ; 0.7)\end{array}$ & $\begin{array}{r}0.7 \\
(0.7 ; 0.7)\end{array}$ \\
\hline $\begin{array}{l}\text { Sugar free beverage } \\
\text { (freq./week) }\end{array}$ & $\begin{array}{r}2.6 \\
(2.3 ; 2.8)\end{array}$ & $\begin{array}{r}2.6 \\
(2.4 ; 2.8)\end{array}$ & $\begin{array}{r}2.8 \\
(2.6 ; 3.1)\end{array}$ & $\begin{array}{l}1.8 * * * \\
(1.6 ; 2.0)\end{array}$ & $\begin{array}{r}0.9 \\
(0.8 ; 1.0)\end{array}$ & $\begin{array}{r}1.0 \\
(0.8 ; 1.1)\end{array}$ & $\begin{array}{r}1.6 \\
(1.4 ; 1.8)\end{array}$ & $\begin{array}{r}1.7 \\
(1.6 ; 1.9)\end{array}$ & $\begin{array}{r}2.8 \\
(2.4 ; 3.1)\end{array}$ & $\begin{array}{r}2.4^{-} \\
(2.1 ; 2.7)\end{array}$ & $\begin{array}{r}2.0 \\
(1.9 ; 2.1)\end{array}$ & $\begin{array}{l}1.8 * * * \\
(1.7 ; 1.9)\end{array}$ \\
\hline $\begin{array}{l}\text { Soft drinks, sugar } \\
\text { sweetened (freq./week) }\end{array}$ & $\begin{array}{r}1.9 \\
(1.7 ; 2.0)\end{array}$ & $\begin{array}{r}1.8 \\
(1.6 ; 1.9)\end{array}$ & $\begin{array}{r}1.6 \\
(1.5 ; 1.8)\end{array}$ & $\begin{array}{r}1.3^{* *} \\
(1.2 ; 1.4)\end{array}$ & $\begin{array}{r}1.1 \\
(1.0 ; 1.3)\end{array}$ & $\begin{array}{l}1.6 * * * \\
(1.4 ; 1.7)\end{array}$ & $\begin{array}{r}2.2 \\
(2.0 ; 2.4)\end{array}$ & $\begin{array}{r}2.0 \\
(1.8 ; 2.2)\end{array}$ & $\begin{array}{r}1.7 \\
(1.5 ; 2.0)\end{array}$ & $\begin{array}{r}1.8 \\
(1.6 ; 2.1)\end{array}$ & $\begin{array}{r}1.5 \\
(1.5 ; 1.6)\end{array}$ & $\begin{array}{r}1.6 \\
(1.5 ; 1.7)\end{array}$ \\
\hline $\begin{array}{l}\text { Energy drinks } \\
\text { (freq./week) }\end{array}$ & $\begin{array}{r}0.7 \\
(0.5 ; 0.9)\end{array}$ & $\begin{array}{r}1.3^{* *} \\
(1.0 ; 1.6)\end{array}$ & $\begin{array}{r}0.8 \\
(0.6 ; 1.1)\end{array}$ & $\begin{array}{r}0.7 \\
(0.6 ; 0.9)\end{array}$ & $\begin{array}{r}0.9 \\
(0.7 ; 1.0)\end{array}$ & $\begin{array}{l}1.9 * * * \\
(1.4 ; 2.3)\end{array}$ & $\begin{array}{r}1.0 \\
(0.8 ; 1.1)\end{array}$ & $\begin{array}{r}1.0 \\
(0.8 ; 1.2)\end{array}$ & $\begin{array}{r}0.9 \\
(0.7 ; 1.2)\end{array}$ & $\begin{array}{r}1.4^{-} \\
(1.0 ; 1.7)\end{array}$ & $\begin{array}{r}0.8 \\
(0.7 ; 0.9)\end{array}$ & $\begin{array}{l}1.4^{* * *} \\
(1.2 ; 1.6)\end{array}$ \\
\hline
\end{tabular}

Note: $\quad-p<0.10, * p<0.05, * * p<0.01, * * * p<0.001$ : Differences between survey years 2011 and 2014 using t-tests and Chi-square tests.

Excluding fruit juice.

@Weighted according to population size in the five Nordic countries. 
Table 98: Mean (95\% Cl) intake of selected foods among children in the Nordic countries. NORMO 2011 and 2014

\begin{tabular}{|c|c|c|c|c|c|c|c|c|c|c|c|c|}
\hline & \multicolumn{2}{|c|}{ Denmark } & \multicolumn{2}{|c|}{ Finland } & \multicolumn{2}{|c|}{ Sweden } & \multicolumn{2}{|c|}{ Iceland } & \multicolumn{2}{|c|}{ Norway } & \multicolumn{2}{|c|}{ Nordic region@ } \\
\hline & $\begin{array}{r}2011 \\
(n=608)\end{array}$ & $\begin{array}{r}2014 \\
(n=592)\end{array}$ & $\begin{array}{r}2011 \\
(n=496)\end{array}$ & $\begin{array}{r}2014 \\
(n=467)\end{array}$ & $\begin{array}{r}2011 \\
(n=492)\end{array}$ & $\begin{array}{r}2014 \\
(n=481)\end{array}$ & $\begin{array}{r}2011 \\
(n=508)\end{array}$ & $\begin{array}{r}2014 \\
(n=493)\end{array}$ & $\begin{array}{r}2011 \\
(n=349)\end{array}$ & $\begin{array}{r}2014 \\
(n=338)\end{array}$ & $\begin{array}{r}2011 \\
(n=2,459)\end{array}$ & $\begin{array}{r}2014 \\
(n=2,377)\end{array}$ \\
\hline $\begin{array}{l}\text { Rye bread } \\
\text { (slices/day) }\end{array}$ & $\begin{array}{r}2.1 \\
(2.0 ; 2.2)\end{array}$ & $\begin{array}{r}1.8^{* * *} \\
(1.7 ; 1.9)\end{array}$ & $\begin{array}{r}1.9 \\
(1.7 ; 2.0)\end{array}$ & $\begin{array}{r}1.8 \\
(1.7 ; 2.0)\end{array}$ & - & - & $\begin{array}{r}0.4 \\
(0.4 ; 0.5)\end{array}$ & $\begin{array}{r}0.5 \\
(0.4 ; 0.5)\end{array}$ & $\begin{array}{r}0.5 \\
(0.3 ; 0.6)\end{array}$ & $\begin{array}{r}0.9 * * * \\
(0.7 ; 1.1)\end{array}$ & $\begin{array}{r}1.0 \\
(0.9 ; 1.0)\end{array}$ & $\begin{array}{r}1.0 \\
(0.9 ; 1.0)\end{array}$ \\
\hline $\begin{array}{l}\text { Wholemeal bread } \\
\text { (slices/day) }\end{array}$ & $\begin{array}{r}1.2 \\
(1.1 ; 1.3)\end{array}$ & $\begin{array}{r}1.5^{* * *} \\
(1.3 ; 1.6)\end{array}$ & $\begin{array}{r}1.4 \\
(1.3 ; 1.6)\end{array}$ & $\begin{array}{r}1.2^{* *} \\
(1.0 ; 1.3)\end{array}$ & $\begin{array}{r}1.1 \\
(1.0 ; 1.3)\end{array}$ & $\begin{array}{r}1.3^{*} \\
(1.2 ; 1.4)\end{array}$ & $\begin{array}{r}1.5 \\
(1.4 ; 1.6)\end{array}$ & $\begin{array}{r}1.2^{* * *} \\
(1.1 ; 1.3)\end{array}$ & $\begin{array}{r}3.7 \\
(3.5 ; 3.9)\end{array}$ & $\begin{array}{r}2.9 * * * \\
(2.6 ; 3.1)\end{array}$ & $\begin{array}{r}1.8 \\
(1.7 ; 1.8)\end{array}$ & $\begin{array}{r}1.6 * * \\
(1.6 ; 1.7)\end{array}$ \\
\hline $\begin{array}{l}\text { White bread } \\
\text { (slices/day) }\end{array}$ & $\begin{array}{r}0.6 \\
(0.5 ; 0.7)\end{array}$ & $\begin{array}{r}0.5^{*} \\
(0.4 ; 0.6)\end{array}$ & $\begin{array}{r}0.6 \\
(0.5 ; 0.6)\end{array}$ & $\begin{array}{c}0.7^{-} \\
(0.6 ; 0.8)\end{array}$ & $\begin{array}{r}0.8 \\
(0.7 ; 0.9)\end{array}$ & $\begin{array}{r}1.0^{*} \\
(0.9 ; 1.1)\end{array}$ & $\begin{array}{r}0.1 \\
(0.1 ; 0.1)\end{array}$ & $\begin{array}{r}0.1 \\
(0.1 ; 0.1)\end{array}$ & $\begin{array}{r}0.4 \\
(0.3 ; 0.5)\end{array}$ & $\begin{array}{r}0.5 \\
(0.4 ; 0.6)\end{array}$ & $\begin{array}{r}0.6 \\
(0.6 ; 0.7)\end{array}$ & $\begin{array}{r}0.7^{* *} \\
(0.7 ; 0.7)\end{array}$ \\
\hline $\begin{array}{l}\text { Hard bread } \\
\text { (slices/day) }\end{array}$ & $\begin{array}{r}0.3 \\
(0.3 ; 0.4)\end{array}$ & $\begin{array}{r}0.3 \\
(0.3 ; 0.3)\end{array}$ & $\begin{array}{r}0.6 \\
(0.6 ; 0.7)\end{array}$ & $\begin{array}{r}0.7 \\
(0.7 ; 0.8)\end{array}$ & $\begin{array}{r}0.7 \\
(0.6 ; 0.8)\end{array}$ & $\begin{array}{r}0.6 \\
(0.6 ; 0.7)\end{array}$ & $\begin{array}{r}0.3 \\
(0.3 ; 0.4)\end{array}$ & $\begin{array}{r}0.3 \\
(0.2 ; 0.3)\end{array}$ & $\begin{array}{r}0.6 \\
(0.5 ; 0.7)\end{array}$ & $\begin{array}{r}0.6 \\
(0.5 ; 0.7)\end{array}$ & $\begin{array}{r}0.6 \\
(0.5 ; 0.6)\end{array}$ & $\begin{array}{r}0.5 \\
(0.5 ; 0.6)\end{array}$ \\
\hline $\begin{array}{l}\text { Vegetables } \\
\text { (freq./day) }\end{array}$ & $\begin{array}{r}1.4 \\
(1.3 ; 1.5)\end{array}$ & $\begin{array}{r}1.5^{*} \\
(1.5 ; 1.6)\end{array}$ & $\begin{array}{r}1.4 \\
(1.4 ; 1.5)\end{array}$ & $\begin{array}{r}1.6^{*} \\
(1.5 ; 1.7)\end{array}$ & $\begin{array}{r}1.4 \\
(1.3 ; 1.5)\end{array}$ & $\begin{array}{r}1.5^{* *} \\
(1.5 ; 1.6)\end{array}$ & $\begin{array}{r}1.2 \\
(1.2 ; 1.3)\end{array}$ & $\begin{array}{r}1.2 \\
(1.1 ; 1.2)\end{array}$ & $\begin{array}{r}1.3 \\
(1.2 ; 1.4)\end{array}$ & $\begin{array}{r}1.1^{* *} \\
(1.1 ; 1.2)\end{array}$ & $\begin{array}{r}1.4 \\
(1.4 ; 1.4)\end{array}$ & $\begin{array}{r}1.5^{* *} \\
(1.4 ; 1.5)\end{array}$ \\
\hline $\begin{array}{l}\text { Fruit }^{\epsilon} \\
\text { (freq./day) }\end{array}$ & $\begin{array}{r}1.7 \\
(1.6 ; 1.8)\end{array}$ & $\begin{array}{r}1.6^{-} \\
(1.5 ; 1.7)\end{array}$ & $\begin{array}{r}1.2 \\
(1.1 ; 1.2)\end{array}$ & $\begin{array}{r}1.2 \\
(1.1 ; 1.3)\end{array}$ & $\begin{array}{r}1.4 \\
(1.4 ; 1.5)\end{array}$ & $\begin{array}{r}1.5 \\
(1.4 ; 1.6)\end{array}$ & $\begin{array}{r}1.4 \\
(1.3 ; 1.5)\end{array}$ & $\begin{array}{r}1.4 \\
(1.4 ; 1.5)\end{array}$ & $\begin{array}{r}1.2 \\
(1.1 ; 1.3)\end{array}$ & $\begin{array}{r}1.3^{*} \\
(1.3 ; 1.4)\end{array}$ & $\begin{array}{r}1.4 \\
(1.3 ; 1.4)\end{array}$ & $\begin{array}{r}1.4 \\
(1.4 ; 1.5)\end{array}$ \\
\hline $\begin{array}{l}\text { Fruit juice } \\
\text { (freq./day) }\end{array}$ & $\begin{array}{r}0.3 \\
(0.3 ; 0.3)\end{array}$ & $\begin{array}{r}0.3 \\
(0.2 ; 0.3)\end{array}$ & $\begin{array}{r}0.4 \\
(0.3 ; 0.4)\end{array}$ & $\begin{array}{r}0.3 \\
(0.3 ; 0.4)\end{array}$ & $\begin{array}{r}0.4 \\
(0.3 ; 0.4)\end{array}$ & $\begin{array}{r}0.4 \\
(0.3 ; 0.4)\end{array}$ & $\begin{array}{r}0.6 \\
(0.5 ; 0.6)\end{array}$ & $\begin{array}{r}0.5^{* * *} \\
(0.4 ; 0.5)\end{array}$ & $\begin{array}{r}0.6 \\
(0.5 ; 0.6)\end{array}$ & $\begin{array}{r}0.5 \\
(0.5 ; 0.6)\end{array}$ & $\begin{array}{r}0.4 \\
(0.4 ; 0.4)\end{array}$ & $\begin{array}{r}0.4^{* *} \\
(0.3 ; 0.4)\end{array}$ \\
\hline $\begin{array}{l}\text { Fish } \\
\text { (freq./week) }\end{array}$ & $\begin{array}{r}0.8 \\
(0.7 ; 0.9)\end{array}$ & $\begin{array}{r}1.0^{*} \\
(0.8 ; 1.1)\end{array}$ & $\begin{array}{r}1.3 \\
(1.2 ; 1.4)\end{array}$ & $\begin{array}{r}1.5^{*} \\
(1.4 ; 1.5)\end{array}$ & $\begin{array}{r}1.4 \\
(1.3 ; 1.5)\end{array}$ & $\begin{array}{r}1.5^{-} \\
(1.4 ; 1.6)\end{array}$ & $\begin{array}{r}2.4 \\
(2.3 ; 2.4)\end{array}$ & $\begin{array}{r}2.6 * \\
(2.4 ; 2.8)\end{array}$ & $\begin{array}{r}1.7 \\
(1.4 ; 1.9)\end{array}$ & $\begin{array}{r}1.8 \\
(1.6 ; 1.9)\end{array}$ & $\begin{array}{r}1.3 \\
(1.3 ; 1.4)\end{array}$ & $\begin{array}{r}1.4^{* * *} \\
(1.4 ; 1.5)\end{array}$ \\
\hline $\begin{array}{l}\text { French fries } \\
\text { (freq./month) }\end{array}$ & $\begin{array}{r}3.5 \\
(3.1 ; 3.8)\end{array}$ & $\begin{array}{r}3.5 \\
(3.1 ; 3.8)\end{array}$ & $\begin{array}{r}2.5 \\
(2.2 ; 2.8)\end{array}$ & $\begin{array}{r}2.4 \\
(2.2 ; 2.6)\end{array}$ & $\begin{array}{r}2.5 \\
(2.3 ; 2.7)\end{array}$ & $\begin{array}{r}3.3^{* * *} \\
(3.1 ; 3.6)\end{array}$ & $\begin{array}{r}2.4 \\
(2.2 ; 2.7)\end{array}$ & $\begin{array}{r}2.6 \\
(2.4 ; 2.9)\end{array}$ & $\begin{array}{r}2.6 \\
(2.3 ; 2.9)\end{array}$ & $\begin{array}{r}2.7 \\
(2.2 ; 3.2)\end{array}$ & $\begin{array}{r}2.7 \\
(2.6 ; 2.9)\end{array}$ & $\begin{array}{r}3.0^{* *} \\
(2.9 ; 3.2)\end{array}$ \\
\hline $\begin{array}{l}\text { Sausage } \\
\text { (freq./month) }\end{array}$ & $\begin{array}{r}1.5 \\
(1.3 ; 1.7)\end{array}$ & $\begin{array}{r}1.4 \\
(1.2 ; 1.6)\end{array}$ & $\begin{array}{r}3.4 \\
(3.2 ; 3.7)\end{array}$ & $\begin{array}{r}3.2 \\
(3.0 ; 3.40)\end{array}$ & $\begin{array}{r}5.4 \\
(5.0 ; 5.8)\end{array}$ & $\begin{array}{r}4.9^{-} \\
(4.775 .2)\end{array}$ & $\begin{array}{r}2.9 \\
(2.7 ; 3.1)\end{array}$ & $\begin{array}{r}2.8 \\
(2.3 ; 3.4)\end{array}$ & $\begin{array}{r}3.9 \\
(3.5 ; 4.3)\end{array}$ & $\begin{array}{r}2.8 * * * \\
(2.5 ; 3.2)\end{array}$ & $\begin{array}{r}3.7 \\
(3.6 ; 3.9)\end{array}$ & $\begin{array}{r}3.3 \\
(3.2 ; 3.5)\end{array}$ \\
\hline $\begin{array}{l}\text { Chocolate and candy } \\
\text { (freq./month) }\end{array}$ & $\begin{array}{r}9.2 \\
(8.5 ; 10.0)\end{array}$ & $\begin{array}{r}8.5 \\
(8.0 ; 9.0)\end{array}$ & $\begin{array}{r}8.7 \\
(8.0 ; 9.3)\end{array}$ & $\begin{array}{r}7.3^{* *} \\
(6.8 ; 7.8)\end{array}$ & $\begin{array}{r}5.9 \\
(5.5 ; 6.2)\end{array}$ & $\begin{array}{r}6.0 \\
(5.6 ; 6.3)\end{array}$ & $\begin{array}{r}6.6 \\
(6.2 ; 7.0)\end{array}$ & $\begin{array}{r}7.4^{*} \\
(6.9 ; 7.8)\end{array}$ & $\begin{array}{r}7.4 \\
(6.6 ; 8.2)\end{array}$ & $\begin{array}{r}7.6 \\
(6.9 ; 8.3)\end{array}$ & $\begin{array}{r}7.5 \\
(7.377 .8)\end{array}$ & $\begin{array}{r}7.1^{*} \\
(6.9 ; 7.4)\end{array}$ \\
\hline $\begin{array}{l}\text { Cake } \\
\text { (freq./month) }\end{array}$ & $\begin{array}{r}7.8 \\
(7.2 ; 8.3)\end{array}$ & $\begin{array}{r}7.5 \\
(6.9 ; 8.1)\end{array}$ & $\begin{array}{r}8.5 \\
(7.8 ; 9.2)\end{array}$ & $\begin{array}{r}7.8 \\
(7.2 ; 8.4)\end{array}$ & $\begin{array}{r}6.9 \\
(6.377 .5)\end{array}$ & $\begin{array}{r}7.5 \\
(6.6 ; 8.4)\end{array}$ & $\begin{array}{r}11.6 \\
(10.7 ; 12.5)\end{array}$ & $\begin{array}{r}11.3 \\
(10.4 ; 12.1)\end{array}$ & $\begin{array}{r}5.1 \\
(4.7 ; 5.6)\end{array}$ & $\begin{array}{r}4.7 \\
(4.2 ; 5.1)\end{array}$ & $\begin{array}{r}7.1 \\
(6.8 ; 7.4)\end{array}$ & $\begin{array}{r}7.0 \\
(6.777 .3)\end{array}$ \\
\hline
\end{tabular}




\begin{tabular}{|c|c|c|c|c|c|c|c|c|c|c|c|c|}
\hline & \multicolumn{2}{|c|}{ Denmark } & \multicolumn{2}{|c|}{ Finland } & \multicolumn{2}{|c|}{ Sweden } & \multicolumn{2}{|c|}{ Iceland } & \multicolumn{2}{|c|}{ Norway } & \multicolumn{2}{|c|}{ Nordic region@ } \\
\hline & $\begin{array}{r}2011 \\
(n=608)\end{array}$ & $\begin{array}{r}2014 \\
(n=592)\end{array}$ & $\begin{array}{r}2011 \\
(n=496)\end{array}$ & $\begin{array}{r}2014 \\
(n=467)\end{array}$ & $\begin{array}{r}2011 \\
(n=492)\end{array}$ & $\begin{array}{r}2014 \\
(n=481)\end{array}$ & $\begin{array}{r}2011 \\
(n=508)\end{array}$ & $\begin{array}{r}2014 \\
(n=493)\end{array}$ & $\begin{array}{r}2011 \\
(n=349)\end{array}$ & $\begin{array}{r}2014 \\
(n=338)\end{array}$ & $\begin{array}{r}2011 \\
(n=2,459)\end{array}$ & $\begin{array}{r}2014 \\
(n=2,377)\end{array}$ \\
\hline \multicolumn{13}{|l|}{ Table 98 continued } \\
\hline $\begin{array}{l}\text { Cheese } \\
\text { (freq./day) }\end{array}$ & $\begin{array}{r}0.3 \\
(0.3 ; 0.3)\end{array}$ & $\begin{array}{r}0.2^{* *} \\
(0.2 ; 0.3)\end{array}$ & $\begin{array}{r}0.7 \\
(0.6 ; 0.7)\end{array}$ & $\begin{array}{r}0.5^{*} \\
(0.5 ; 0.6)\end{array}$ & $\begin{array}{r}0.6 \\
(0.5 ; 0.6)\end{array}$ & $\begin{array}{r}0.5 \\
(0.5 ; 0.6)\end{array}$ & $\begin{array}{r}0.5 \\
(0.5 ; 0.6)\end{array}$ & $\begin{array}{r}0.5 \\
(0.4 ; 0.5)\end{array}$ & $\begin{array}{r}0.7 \\
(0.6 ; 0.7)\end{array}$ & $\begin{array}{r}0.7 \\
(0.7 ; 0.8)\end{array}$ & $\begin{array}{r}0.5 \\
(0.5 ; 0.6)\end{array}$ & $\begin{array}{r}0.5^{*} \\
(0.5 ; 0.5)\end{array}$ \\
\hline $\begin{array}{l}\text { Sugar free beverage } \\
\text { (freq./week) }\end{array}$ & $\begin{array}{r}1.8 \\
(1.5 ; 2.0)\end{array}$ & $\begin{array}{r}1.5 \\
(1.3 ; 1.8)\end{array}$ & $\begin{array}{r}1.7 \\
(1.3 ; 2.0)\end{array}$ & $\begin{array}{r}1.0^{* *} \\
(0.8 ; 1.2)\end{array}$ & $\begin{array}{r}0.6 \\
(0.4 ; 0.7)\end{array}$ & $\begin{array}{r}0.5 \\
(0.4 ; 0.7)\end{array}$ & $\begin{array}{r}1.4 \\
(1.2 ; 1.7)\end{array}$ & $\begin{array}{r}0.9^{* * *} \\
(0.7 ; 1.0)\end{array}$ & $\begin{array}{r}2.3 \\
(1.9 ; 2.7)\end{array}$ & $\begin{array}{r}1.7^{*} \\
(1.4 ; 2.0)\end{array}$ & $\begin{array}{r}1.4 \\
(1.3 ; 1.6)\end{array}$ & $\begin{array}{r}1.1 * * * \\
(1.0 ; 1.2)\end{array}$ \\
\hline $\begin{array}{l}\text { Soft drinks, sugar sweetened } \\
\text { (freq./week) }\end{array}$ & $\begin{array}{r}1.7 \\
(1.4 ; 1.9)\end{array}$ & $\begin{array}{r}1.5 \\
(1.3 ; 1.7)\end{array}$ & $\begin{array}{r}2.3 \\
(2.0 ; 2.6)\end{array}$ & $\begin{array}{r}2.2 \\
(1.9 ; 2.4)\end{array}$ & $\begin{array}{r}1.5 \\
(1.3 ; 1.6)\end{array}$ & $\begin{array}{r}1.5 \\
(1.3 ; 1.7)\end{array}$ & $\begin{array}{r}1.4 \\
(1.3 ; 1.6)\end{array}$ & $\begin{array}{r}1.1^{* * *} \\
(1.0 ; 1.2)\end{array}$ & $\begin{array}{r}1.5 \\
(1.3 ; 1.8)\end{array}$ & $\begin{array}{r}1.3 \\
(1.2 ; 1.5)\end{array}$ & $\begin{array}{r}1.7 \\
(1.6 ; 1.8)\end{array}$ & $\begin{array}{r}1.6 \\
(1.5 ; 1.7)\end{array}$ \\
\hline
\end{tabular}

$\quad \quad \quad \quad p<0.10,{ }^{*} p<0.05,{ }^{* *} p<0.01,{ }^{* * *} p<0.001$ : Differences between survey years 2011 and 2014 using t-tests and Chi-square tests.

${ }^{\mathrm{f} E x c l u d i n g}$ fruit juice.

@Weighted according to population size in the five Nordic countries.

Table 99: Proportion of adults using the different types of spread, 2011 and 2014

\begin{tabular}{|c|c|c|c|c|c|c|c|c|c|c|c|c|}
\hline & \multicolumn{2}{|c|}{ Denmark } & \multicolumn{2}{|c|}{ Finland } & \multicolumn{2}{|c|}{ Sweden } & \multicolumn{2}{|c|}{ Iceland } & \multicolumn{2}{|c|}{ Norway } & \multicolumn{2}{|c|}{ Nordic region } \\
\hline & $2011 \%$ & $2014 \%$ & $2011 \%$ & $2014 \%$ & $2011 \%$ & $2014 \%$ & $2011 \%$ & $2014 \%$ & $2011 \%$ & $2014 \%$ & $2011 \%$ & $2014 \%$ \\
\hline Butter & 15.3 & 16.0 & 9.2 & 7.2 & 4.6 & 3.8 & 11.4 & 25.7 & 9.0 & 11.9 & 8.9 & 9.0 \\
\hline Oil-butter spreads & 38.0 & 41.6 & 35.6 & 42.1 & 39.4 & 52.8 & 47.7 & 45.8 & 29.8 & 30.9 & 36.5 & 43.7 \\
\hline Margarine & 2.6 & 2.4 & 12.2 & 17.1 & 16.0 & 13.0 & 5.4 & 2.8 & 7.5 & 6.5 & 10.5 & 10.2 \\
\hline Low fat margarine & 4.2 & 2.4 & 16.9 & 13.0 & 25.5 & 17.8 & 21.3 & 9.7 & 24.1 & 22.3 & 18.7 & 14.4 \\
\hline "Becel Pro-Activ" & 2.2 & 1.5 & 17.8 & 13.1 & 3.2 & 2.1 & 0.3 & 0.3 & 2.5 & 3.6 & 5.9 & 4.5 \\
\hline Do not use spread on bread & 37.7 & 36.1 & 8.3 & 7.4 & 11.2 & 10.5 & 13.9 & 15.7 & 27.2 & 24.8 & 19.6 & 18.2 \\
\hline
\end{tabular}

Note: @Weighted according to population size in the five Nordic countries. 
Table 100: Proportion of adults using the different types of spread/grease for cooking, 2011 and 2014

\begin{tabular}{|c|c|c|c|c|c|c|c|c|c|c|c|c|}
\hline & \multicolumn{2}{|c|}{ Denmark } & \multicolumn{2}{|c|}{ Finland } & \multicolumn{2}{|c|}{ Sweden } & \multicolumn{2}{|c|}{ Iceland } & \multicolumn{2}{|c|}{ Norway } & \multicolumn{2}{|c|}{ Nordic region } \\
\hline & $2011 \%$ & $2014 \%$ & $2011 \%$ & $2014 \%$ & $2011 \%$ & $2014 \%$ & $2011 \%$ & $2014 \%$ & $2011 \%$ & $2014 \%$ & $2011 \%$ & $2014 \%$ \\
\hline Butter & 3.6 & 4.2 & 18.6 & 15.6 & 22.6 & 22.4 & 4.4 & 10.0 & 8.6 & 10.0 & 14.7 & 14.5 \\
\hline Oil-butter spreads & 2.1 & 1.9 & 8.3 & 9.6 & 0.9 & 1.7 & 0.7 & 0.8 & 2.8 & 3.4 & 3.1 & 3.7 \\
\hline Margarine & 15.7 & 10.4 & 5.5 & 3.6 & 13.1 & 9.1 & 8.8 & 5.8 & 22.7 & 21.1 & 13.9 & 10.6 \\
\hline Use of mixture of oil and butter & 25.5 & 30.1 & 8.9 & 9.3 & 32.4 & 34.6 & 2.2 & 6.7 & 13.3 & 14.3 & 21.9 & 24.1 \\
\hline Oil & 53.1 & 53.4 & 58.7 & 61.9 & 31.0 & 32.2 & 83.9 & 76.7 & 52.7 & 51.1 & 46.5 & 47.2 \\
\hline
\end{tabular}

Note: @Weighted according to population size in the five Nordic countries.

Table 101: Proportion of children using the different types of spread, 2011 and 2014

\begin{tabular}{|c|c|c|c|c|c|c|c|c|c|c|c|c|}
\hline & \multicolumn{2}{|c|}{ Denmark } & \multicolumn{2}{|c|}{ Finland } & \multicolumn{2}{|c|}{ Sweden } & \multicolumn{2}{|c|}{ Iceland } & \multicolumn{2}{|c|}{ Norway } & \multicolumn{2}{|c|}{ Nordic region } \\
\hline & $2011 \%$ & $2014 \%$ & $2011 \%$ & $2014 \%$ & $2011 \%$ & $2014 \%$ & $2011 \%$ & $2014 \%$ & $2011 \%$ & $2014 \%$ & $2011 \%$ & $2014 \%$ \\
\hline Butter & 12.4 & 12.1 & 5.4 & 3.7 & 1.7 & 1.9 & 7.2 & 17.2 & 6.5 & 7.0 & 6.0 & 5.8 \\
\hline Oil-butter spreads & 50.5 & 60.9 & 42.6 & 45.7 & 49.4 & 52.5 & 50.4 & 53.9 & 28.9 & 37.7 & 44.0 & 50.0 \\
\hline Margarine & 3.6 & 1.8 & 20.3 & 27.3 & 16.6 & 20.3 & 7.1 & 3.4 & 7.6 & 3.1 & 12.3 & 13.8 \\
\hline Low fat margarine & 7.4 & 3.9 & 10.7 & 17.6 & 25.4 & 20.1 & 21.4 & 12.1 & 28.5 & 24.7 & 18.9 & 16.8 \\
\hline "Becel Pro-Activ" & 1.9 & 1.6 & 19.0 & 4.7 & 1.3 & 0.6 & 2.3 & . & 2.7 & 2.3 & 5.3 & 2.0 \\
\hline Do not use spread on bread & 24.2 & 19.7 & 1.5 & 1.0 & 5.3 & 4.6 & 11.6 & 13.4 & 24.0 & 25.1 & 12.9 & 11.6 \\
\hline
\end{tabular}

Note: @Weighted according to population size in the five Nordic countries.

Table 102: Proportion of children using the different types of spread/grease for cooking, 2011 and 2014

\begin{tabular}{|c|c|c|c|c|c|c|c|c|c|c|c|c|}
\hline & \multicolumn{2}{|c|}{ Denmark } & \multicolumn{2}{|c|}{ Finland } & \multicolumn{2}{|c|}{ Sweden } & \multicolumn{2}{|c|}{ Iceland } & \multicolumn{2}{|c|}{ Norway } & \multicolumn{2}{|c|}{ Nordic region } \\
\hline & $2011 \%$ & $2014 \%$ & $2011 \%$ & $2014 \%$ & $2011 \%$ & $2014 \%$ & $2011 \%$ & $2014 \%$ & $2011 \%$ & $2014 \%$ & $2011 \%$ & $2014 \%$ \\
\hline Butter & 3.6 & $4 \cdot 3$ & 18.6 & 14.9 & 23.6 & 25.4 & . & 7.8 & 6.4 & 5.9 & 14.0 & 14.4 \\
\hline Oil-butter spreads & 2.4 & 0.5 & 9.5 & 7.8 & 0.6 & 0.1 & 1.3 & 1.8 & 3.0 & 5.7 & 3.3 & 2.9 \\
\hline Margarine & 12.8 & 7.3 & 5.5 & 5.5 & 18.4 & 8.0 & 6.4 & 4.2 & 28.6 & 17.0 & 16.6 & 9.2 \\
\hline Use of mixture of oil and butter & 25.6 & 25.8 & 9.1 & 7.6 & 27.8 & 35.4 & 2.9 & 12.4 & 10.9 & 8.7 & 19.7 & 22.0 \\
\hline Oil & 55.8 & 62.0 & 57.3 & 64.3 & 29.6 & 31.1 & 89.4 & 73.8 & 51.0 & 62.7 & 46.5 & 51.5 \\
\hline
\end{tabular}

Note: @Weighted according to population size in the five Nordic countries. 
Physical activity

Adults

Table 103: Level of occupational physical activity during the last 7 days among adults in the Nordic countries. NORMO 2011 and 2014

\begin{tabular}{|c|c|c|c|c|c|c|c|c|c|c|c|c|}
\hline & \multicolumn{2}{|c|}{ Denmark } & \multicolumn{2}{|c|}{ Finland } & \multicolumn{2}{|c|}{ Sweden } & \multicolumn{2}{|c|}{ Iceland } & \multicolumn{2}{|c|}{ Norway } & \multicolumn{2}{|c|}{ Nordic region@ } \\
\hline & $\begin{array}{r}2011 \\
(n=1,789)\end{array}$ & $\begin{array}{r}2014 \\
(n=1,688)\end{array}$ & $\begin{array}{r}2011 \\
(n=1,481)\end{array}$ & $\begin{array}{r}2014 \\
(n=1,262)\end{array}$ & $\begin{array}{r}2011 \\
(n=1,627)\end{array}$ & $\begin{array}{r}2014 \\
(n=1,528)\end{array}$ & $\begin{array}{r}2011 \\
(n=1,799)\end{array}$ & $\begin{array}{r}2014 \\
(n=1,809)\end{array}$ & $\begin{array}{r}2011 \\
(n=915)\end{array}$ & $\begin{array}{r}2014 \\
(n=931)\end{array}$ & $\begin{array}{r}2011 \\
(n=7,611)\end{array}$ & $\begin{array}{r}2014 \\
(n=7,218)\end{array}$ \\
\hline Sedentary & 39.3 & 36.9 & 44.4 & 40.8 & 44.6 & 42.5 & 46.9 & 39.8 & 47.2 & 38.9 & 44.0 & 40.2 \\
\hline Light activity & 31.8 & 25.2 & 22.8 & 22.9 & 24.6 & 27.6 & 26.0 & 27.0 & 21.7 & 21.3 & 25.2 & 24.9 \\
\hline Moderate activity & 21.3 & 30.9 & 22.4 & 24.3 & 22.5 & 23.2 & 18.2 & 23.0 & 19.2 & 26.1 & 21.6 & 25.6 \\
\hline Heavy activity & 7.5 & 7.0 & 10.4 & 12.0 & 8.3 & 6.7 & 8.9 & 10.2 & 11.9 & 13.6 & 9.2 & 9.3 \\
\hline
\end{tabular}

Note: @Weighted according to population size in the five Nordic countries.

Table 104: Level of leisure time physical activity during the last 7 days (\%) among adults in the Nordic countries. NORMO 2011 and 2014

\begin{tabular}{|c|c|c|c|c|c|c|c|c|c|c|c|c|}
\hline & \multicolumn{2}{|c|}{ Denmark } & \multicolumn{2}{|c|}{ Finland } & \multicolumn{2}{|c|}{ Sweden } & \multicolumn{2}{|c|}{ Iceland } & \multicolumn{2}{|c|}{ Norway } & \multicolumn{2}{|c|}{ Nordic region@ } \\
\hline & $\begin{array}{r}2011 \\
(n=2,178)\end{array}$ & $\begin{array}{r}2014 \\
(n=2,004)\end{array}$ & $\begin{array}{r}2011 \\
(n=1,987)\end{array}$ & $\begin{array}{r}2014 \\
(n=1,700)\end{array}$ & $\begin{array}{r}2011 \\
(n=1,862)\end{array}$ & $\begin{array}{r}2014 \\
(n=1,776)\end{array}$ & $\begin{array}{r}2011 \\
(n=1,942)\end{array}$ & $\begin{array}{r}2014 \\
(n=1,969)\end{array}$ & $\begin{array}{r}2011 \\
(n=1,045)\end{array}$ & $\begin{array}{r}2014 \\
(n=1,045)\end{array}$ & $\begin{array}{r}2011 \\
(n=9,014)\end{array}$ & $\begin{array}{r}2014 \\
(n=8,494)\end{array}$ \\
\hline Sedentary & 30.7 & 29.5 & 28.2 & 27.0 & 41.4 & 42.8 & 51.6 & 49.5 & 37.6 & 34.7 & 35.6 & 35.1 \\
\hline Light activity & 48.1 & 41.5 & 40.6 & 44.6 & 40.5 & 36.3 & 29.7 & 29.9 & 41.3 & 37.4 & 42.2 & 39.3 \\
\hline Moderate activity & 16.0 & 22.5 & 25.6 & 21.9 & 12.1 & 11.4 & 11.8 & 12.5 & 11.0 & 16.3 & 15.6 & 17.0 \\
\hline Vigorous activity & 5.2 & 6.4 & 5.7 & 6.5 & 6.0 & 9.5 & 6.9 & 8.1 & 10.1 & 11.6 & 6.6 & 8.6 \\
\hline
\end{tabular}

Note: @Weighted according to population size in the five Nordic countries. 
Smokeless tobacco (snuff)

Table 105: Proportion of snuff users (\%) among adults in the Nordic countries. NORMO 2014

\begin{tabular}{|c|c|c|c|c|c|c|}
\hline & Denmark" & Finland & Sweden & Iceland & Norway & Nordic region@ \\
\hline & 2014 & $2014(n=1,709)$ & $2014(n=1,798)$ & $2014(n=2,004)$ & $2014(n=1,057)$ & $2014(n=6,568)$ \\
\hline Total & - & 4.1 & 17.4 & 7.9 & 19.6 & 14.3 \\
\hline Daily & - & 2.1 & 14.8 & 5.7 & 16.9 & 11.8 \\
\hline
\end{tabular}

Note: $\quad$ \#Questions on smokeless tobacco (snuff) were not included in Denmark because use of smokeless tobacco has not been very prevalent in Denmark so far. @Weighted according to population size in the five Nordic countries.

Household composition

Adults

Table 106: Proportion with an unhealthy diet (\%) among adults in the Nordic countries according to household composition. NORMO 2011 and 2014

\begin{tabular}{|c|c|c|c|c|c|c|c|c|c|c|c|c|}
\hline & \multicolumn{2}{|c|}{ Denmark } & \multicolumn{2}{|c|}{ Finland } & \multicolumn{2}{|c|}{ Sweden } & \multicolumn{2}{|c|}{ Iceland } & \multicolumn{2}{|c|}{ Norway } & \multicolumn{2}{|c|}{ Nordic region@ } \\
\hline & $\begin{array}{r}2011 \\
(n=1,943)\end{array}$ & $\begin{array}{r}2014 \\
(n=1,985)\end{array}$ & $\begin{array}{r}2011 \\
(n=1,966)\end{array}$ & $\begin{array}{r}2014 \\
(n=1,561)\end{array}$ & $\begin{array}{r}2011 \\
(n=1,860)\end{array}$ & $\begin{array}{r}2014 \\
(n=1,732)\end{array}$ & $\begin{array}{r}2011 \\
(n=2,006)\end{array}$ & $\begin{array}{r}2014 \\
(n=1,962)\end{array}$ & $\begin{array}{r}2011 \\
(n=1,058)\end{array}$ & $\begin{array}{r}2014 \\
(n=961)\end{array}$ & $\begin{array}{r}2011 \\
(n=8,833)\end{array}$ & $\begin{array}{r}2014 \\
(n=8,201)\end{array}$ \\
\hline Single & 21.5 & 26.8 & 18.3 & 15.2 & 22.6 & 30.2 & 25.5 & 22.2 & 8.2 & 17.0 & 18.6 & 23.6 \\
\hline Adult(s) living with children & 22.8 & 23.1 & 15.7 & 18.9 & 23.8 & 29.9 & 18.7 & 25.5 & 9.4 & 13.3 & 18.9 & 22.9 \\
\hline Adult(s) living with other adults & 20.2 & 21.2 & 14.7 & 13.0 & 22.3 & 24.4 & 17.5 & 23.6 & 8.5 & 14.3 & 17.4 & 19.5 \\
\hline
\end{tabular}

Note:

@Weighted according to population size in the five Nordic countries. 
Table 107: Proportion of inactive (\%) among adults in the Nordic countries according to household composition. NORMO 2011 and 2014

\begin{tabular}{|c|c|c|c|c|c|c|c|c|c|c|c|c|}
\hline & \multicolumn{2}{|c|}{ Denmark } & \multicolumn{2}{|c|}{ Finland } & \multicolumn{2}{|c|}{ Sweden } & \multicolumn{2}{|c|}{ Iceland } & \multicolumn{2}{|c|}{ Norway } & \multicolumn{2}{|c|}{ Nordic region@ } \\
\hline & $\begin{array}{r}2011 \\
(n=2,093)\end{array}$ & $\begin{array}{r}2014 \\
(n=1,900)\end{array}$ & $\begin{array}{r}2011 \\
(n=1,817)\end{array}$ & $\begin{array}{r}2014 \\
(n=1,637)\end{array}$ & $\begin{array}{r}2011 \\
(n=1,772)\end{array}$ & $\begin{array}{r}2014 \\
(n=1,632)\end{array}$ & $\begin{array}{r}2011 \\
(n=1,864)\end{array}$ & $\begin{array}{r}2014 \\
(n=1,916)\end{array}$ & $\begin{array}{r}2011 \\
(n=1,011)\end{array}$ & $\begin{array}{r}2014 \\
(n=945)\end{array}$ & $\begin{array}{r}2011 \\
(n=8,557)\end{array}$ & $\begin{array}{r}2014 \\
(n=8,030)\end{array}$ \\
\hline Single & 39.3 & 38.1 & 27.2 & 27.9 & 28.0 & 31.1 & 40.5 & 37.0 & 44.5 & 50.0 & 33.5 & 35.0 \\
\hline Adult(s) living with children & 38.3 & 35.2 & 30.8 & 32.2 & 34.5 & 28.9 & 33.1 & 35.2 & 45.2 & 45.8 & 36.7 & 34.4 \\
\hline Adult(s) living with other adults & 30.4 & 32.4 & 26.8 & 27.5 & 29.2 & 29.6 & 33.8 & 34.6 & 36.1 & 42.5 & 30.3 & 32.3 \\
\hline
\end{tabular}

Note: @Weighted according to population size in the five Nordic countries.

Table 108: Proportion with high recreational screen time (> 4h/d) (\%) among adults in the Nordic countries according to household composition. NORMO 2011 and 2014

\begin{tabular}{|c|c|c|c|c|c|c|c|c|c|c|c|c|}
\hline & \multicolumn{2}{|c|}{ Denmark } & \multicolumn{2}{|c|}{ Finland } & \multicolumn{2}{|c|}{ Sweden } & \multicolumn{2}{|c|}{ Iceland } & \multicolumn{2}{|c|}{ Norway } & \multicolumn{2}{|c|}{ Nordic region@ } \\
\hline & $\begin{array}{r}2011 \\
(n=2,158)\end{array}$ & $\begin{array}{r}2014 \\
(n=1,848)\end{array}$ & $\begin{array}{r}2011 \\
(n=1,935)\end{array}$ & $\begin{array}{r}2014 \\
(n=1,579)\end{array}$ & $\begin{array}{r}2011 \\
(n=1,845)\end{array}$ & $\begin{array}{r}2014 \\
(n=1,769)\end{array}$ & $\begin{array}{r}2011 \\
(n=1,905)\end{array}$ & $\begin{array}{r}2014 \\
(n=1,889)\end{array}$ & $\begin{array}{r}2011 \\
(n=1,030)\end{array}$ & $\begin{array}{r}2014 \\
(n=1,007)\end{array}$ & $\begin{array}{r}2011 \\
(n=8,873)\end{array}$ & $\begin{array}{r}2014 \\
(n=8,092)\end{array}$ \\
\hline Single & 38.7 & 41.0 & 30.7 & 40.9 & 31.0 & 31.6 & 27.8 & 32.6 & 43.0 & 41.9 & 34.7 & 37.2 \\
\hline Adult(s) living with children & 24.9 & 25.7 & 26.8 & 30.1 & 20.5 & 17.9 & 17.7 & 22.9 & 30.1 & 21.6 & 24.4 & 22.7 \\
\hline Adult(s) living with other adults & 30.0 & 37.0 & 25.7 & 36.3 & 26.8 & 28.0 & 28.1 & 29.7 & 39.8 & 35.3 & 29.7 & 33.1 \\
\hline
\end{tabular}

Note: @Weighted according to population size in the five Nordic countries.

Table 109: Prevalence of OW/OB (\%) among adults in the Nordic countries according to household composition. NORMO 2011 and 2014

\begin{tabular}{|c|c|c|c|c|c|c|c|c|c|c|c|c|}
\hline & \multicolumn{2}{|c|}{ Denmark } & \multicolumn{2}{|c|}{ Finland } & \multicolumn{2}{|c|}{ Sweden } & \multicolumn{2}{|c|}{ Iceland } & \multicolumn{2}{|c|}{ Norway } & \multicolumn{2}{|c|}{ Nordic region@ } \\
\hline & $\begin{array}{c}2011 \\
(n=2,158)\end{array}$ & $\begin{array}{c}2014 \\
(n=1,975)\end{array}$ & $\begin{array}{c}2011 \\
(\mathrm{n}=1,980)\end{array}$ & $\begin{array}{c}2014 \\
(n=1,696)\end{array}$ & $\begin{array}{c}2011 \\
(n=1,837)\end{array}$ & $\begin{array}{c}2014 \\
(n=1,761)\end{array}$ & $\begin{array}{c}2011 \\
(n=1,942)\end{array}$ & $\begin{array}{c}2014 \\
(n=1,939)\end{array}$ & $\begin{array}{c}2011 \\
(n=1,033)\end{array}$ & $\begin{array}{c}2014 \\
(n=1,015)\end{array}$ & $\begin{array}{c}2011 \\
(\mathrm{n}=8,950)\end{array}$ & $\begin{array}{c}2014 \\
(n=8,386)\end{array}$ \\
\hline Single & 47.0 & 49.8 & 50.0 & 49.6 & 50.7 & 40.3 & 52.5 & 66.9 & 52.6 & 53.0 & 50.1 & 46.8 \\
\hline Adult(s) living with children & 45.5 & 47.9 & 44.8 & 50.4 & 42.2 & 47.3 & 59.2 & 60.3 & 49.8 & 53.3 & 45.2 & 49.5 \\
\hline Adult(s) living with other adults & 46.9 & 46.2 & 49.5 & 45.7 & 46.4 & 44.6 & $53 \cdot 3$ & 56.0 & 41.4 & 40.4 & 46.4 & 44.5 \\
\hline
\end{tabular}

Note: @Weighted according to population size in the five Nordic countries. 
Table 110: Prevalence of OB (\%) among adults in the Nordic countries according to household composition. NORMO 2011 and 2014

\begin{tabular}{|c|c|c|c|c|c|c|c|c|c|c|c|c|}
\hline & \multicolumn{2}{|c|}{ Denmark } & \multicolumn{2}{|c|}{ Finland } & \multicolumn{2}{|c|}{ Sweden } & \multicolumn{2}{|c|}{ Iceland } & \multicolumn{2}{|c|}{ Norway } & \multicolumn{2}{|c|}{ Nordic region@ } \\
\hline & $\begin{array}{r}2011 \\
(n=2,158)\end{array}$ & $\begin{array}{r}2014 \\
(n=1,975)\end{array}$ & $\begin{array}{r}2011 \\
(n=1,980)\end{array}$ & $\begin{array}{r}2014 \\
(n=1,696)\end{array}$ & $\begin{array}{r}2011 \\
(n=1,837)\end{array}$ & $\begin{array}{r}2014 \\
(n=1,761)\end{array}$ & $\begin{array}{r}2011 \\
(n=1,942)\end{array}$ & $\begin{array}{r}2014 \\
(n=1,939)\end{array}$ & $\begin{array}{r}2011 \\
(n=1,033)\end{array}$ & $\begin{array}{r}2014 \\
(n=1,015)\end{array}$ & $\begin{array}{r}2011 \\
(n=8,950)\end{array}$ & $\begin{array}{r}2014 \\
(n=8,386)\end{array}$ \\
\hline Single & 12.0 & 16.4 & 16.6 & 16.5 & 15.4 & 10.6 & 20.5 & 28.9 & 9.6 & 12.8 & 14.0 & 13.6 \\
\hline Adult(s) living with children & 13.7 & 15.3 & 9.1 & 12.8 & 10.0 & 10.6 & 17.9 & 22.6 & 10.8 & 13.7 & 10.9 & 12.9 \\
\hline Adult(s) living with other adults & 12.5 & 14.0 & 12.6 & 13.8 & 10.0 & 9.3 & 16.3 & 15.7 & 7.4 & 13.3 & 10.8 & 12.2 \\
\hline
\end{tabular}

Note: @Weighted according to population size in the five Nordic countries.

Table 111: Proportion of smokers (\%) among adults in the Nordic countries according to household composition. NORMO 2014

\begin{tabular}{|c|c|c|c|c|c|c|}
\hline & $\begin{array}{l}\text { Denmark } \\
2014(n=2,006)\end{array}$ & $\begin{array}{l}\text { Finland } \\
2014(n=1,712)\end{array}$ & $\begin{array}{l}\text { Sweden } \\
2014(n=1,798)\end{array}$ & $\begin{array}{l}\text { Iceland } \\
2014(n=2,006)\end{array}$ & $\begin{array}{l}\text { Norway } \\
2014(n=1,057)\end{array}$ & $\begin{array}{l}\text { Nordic region }{ }^{\circledR} \\
\qquad 2014(n=8,579)\end{array}$ \\
\hline Single & 34.2 & 28.3 & 21.4 & 32.8 & 22.9 & 25.5 \\
\hline Adult(s) living with children & 20.0 & 23.6 & 11.9 & 18.1 & 23.3 & 18.3 \\
\hline Adult(s) living with other adults & 26.6 & 23.3 & 15.5 & 25.1 & 20.9 & 20.9 \\
\hline
\end{tabular}

Note: @Weighted according to population size in the five Nordic countries.

Table 112: Proportion of daily smokers (\%) among adults in the Nordic countries according to household composition. NORMO 2014

\begin{tabular}{|c|c|c|c|c|c|c|}
\hline & Denmark & Finland & Sweden & Iceland & Norway & Nordic region@ \\
\hline & $2014(n=2,006)$ & $2014(n=1,712)$ & $2014(n=1,798)$ & $2014(n=2,006)$ & $2014(n=1,057)$ & $2014(n=8,579)$ \\
\hline Single & 28.1 & 20.8 & 10.7 & 24.5 & 17.4 & 17.4 \\
\hline Adult(s) living with children & 17.6 & 18.3 & 8.9 & 12.7 & 17.4 & 14.4 \\
\hline Adult(s) living with other adults & 22.1 & 16.1 & 9.3 & 16.2 & 10.3 & 14.0 \\
\hline
\end{tabular}

Note: @Weighted according to population size in the five Nordic countries. 
Table 113: Mean total alcohol consumption (freq./week) among adults in the Nordic countries according to household composition. NORMO 2014

\begin{tabular}{|c|c|c|c|c|c|c|}
\hline & Denmark & Finland & Sweden & Iceland & Norway & Nordic region@ \\
\hline & $2014(n=2,007)$ & $2014(n=1,711)$ & $2014(n=1,799)$ & $2014(n=2,004)$ & $2014(n=1,056)$ & $2014(n=8,577)$ \\
\hline Single & 2.9 & 1.6 & 1.5 & 1.2 & 1.4 & 1.7 \\
\hline Adult(s) living with children & 2.0 & 1.4 & 1.4 & 1.1 & 1.3 & 1.5 \\
\hline Adult(s) living with other adults & 2.7 & 1.6 & 1.5 & 1.3 & 1.9 & 1.9 \\
\hline
\end{tabular}

Note: @Weighted according to population size in the five Nordic countries.

Table 114: Proportion of binge drinkers last month (\%) among adults in the Nordic countries according to household composition. NORMO 2014

\begin{tabular}{|c|c|c|c|c|c|c|}
\hline & $\begin{array}{r}\text { Denmark } \\
2014(n=2,007)\end{array}$ & $\begin{array}{r}\text { Finland } \\
2014(n=1,666)\end{array}$ & $\begin{array}{r}\text { Sweden } \\
2014(n=1,650)\end{array}$ & $\begin{array}{r}\text { Iceland } \\
2014(n=1,980)\end{array}$ & $\begin{array}{r}\text { Norway } \\
2014(n=1,025)\end{array}$ & $\begin{array}{l}\text { Nordic region@ } \\
2014(n=8,328)\end{array}$ \\
\hline Single & 50.1 & 58.5 & 51.3 & 34.8 & $44 \cdot 4$ & 51.3 \\
\hline Adult(s) living with children & 44.1 & 36.7 & 31.8 & 39.4 & 50.5 & 39.5 \\
\hline Adult(s) living with other adults & 46.9 & 43.7 & 44.2 & 40.2 & 55.2 & 46.9 \\
\hline
\end{tabular}

Note: @Weighted according to population size in the five Nordic countries. 


\section{Urbanisation}

The urbanisation categories are different for Iceland than for the other Nordic countries. The five categories for Iceland are as follows

in Tables 115-127:

- The capital and suburb (Reykjavík, Kópavogur, Hafnarfjörður, Garðabær, Seltjarnarnes, Álftanes, Mosfellsbær)

- Urban 5,000 or more

- Urban 1,000-4,999

- Urban 200-999

- Country side.

Adults

Table 115: Proportion with an unhealthy diet (\%) among adults in the Nordic countries according to urbanisation. NORMO 2011 and 2014

\begin{tabular}{|c|c|c|c|c|c|c|c|c|c|c|c|c|}
\hline & \multicolumn{2}{|c|}{ Denmark } & \multicolumn{2}{|c|}{ Finland } & \multicolumn{2}{|c|}{ Sweden } & \multicolumn{2}{|c|}{ Iceland } & \multicolumn{2}{|c|}{ Norway } & \multicolumn{2}{|c|}{ Nordic region@ } \\
\hline & $\begin{array}{r}2011 \\
(n=1,935)\end{array}$ & $\begin{array}{r}2014 \\
(n=1,970)\end{array}$ & $\begin{array}{r}2011 \\
(n=1,952)\end{array}$ & $\begin{array}{r}2014 \\
(n=1,547)\end{array}$ & $\begin{array}{r}2011 \\
(n=1,845)\end{array}$ & $\begin{array}{r}2014 \\
(n=1,712)\end{array}$ & $\begin{array}{r}2011 \\
(n=2,003)\end{array}$ & $\begin{array}{r}2014 \\
(n=1,957)\end{array}$ & $\begin{array}{r}2011 \\
(n=1,051)\end{array}$ & $\begin{array}{r}2014 \\
(n=948)\end{array}$ & $\begin{array}{r}2011 \\
(n=8,786)\end{array}$ & $\begin{array}{r}2014 \\
(n=8,134)\end{array}$ \\
\hline The Capital and suburb & 21.7 & 22.2 & 10.5 & 12.7 & 22.2 & 27.3 & 20.3 & 25.6 & 7.9 & 13.3 & 16.7 & 20.6 \\
\hline Large city $>50,000$ inhab. & 17.3 & 19.0 & 14.3 & 14.3 & 21.9 & 28.0 & 18.9 & 22.7 & 6.5 & 12.7 & 17.0 & 20.9 \\
\hline City $20,000-49,999$ inhab. & 19.8 & 22.6 & 15.5 & 12.7 & 23.7 & 31.0 & 14.9 & 22.5 & 14.9 & 15.0 & 19.3 & 22.4 \\
\hline Town 1,000-19, 999 inhab. & 20.6 & 24.5 & 17.1 & 17.0 & 24.1 & 27.8 & 11.2 & 28.3 & 9.2 & 14.4 & 18.0 & 20.5 \\
\hline Country side & 29.1 & 23.6 & 20.9 & 20.0 & 23.4 & 23.8 & 22.4 & 18.8 & 6.6 & 12.9 & 20.2 & 21.3 \\
\hline
\end{tabular}

Note:

@Weighted according to population size in the five Nordic countries. 
Table 116: Proportion of inactive (\%) among adults in the Nordic countries according to urbanisation. NORMO 2011 and 2014

\begin{tabular}{|c|c|c|c|c|c|c|c|c|c|c|c|c|}
\hline & \multicolumn{2}{|c|}{ Denmark } & \multicolumn{2}{|c|}{ Finland } & \multicolumn{2}{|c|}{ Sweden } & \multicolumn{2}{|c|}{ Iceland } & \multicolumn{2}{|c|}{ Norway } & \multicolumn{2}{|c|}{ Nordic region@ } \\
\hline & $\begin{array}{r}2011 \\
(n=2,083)\end{array}$ & $\begin{array}{r}2014 \\
(n=1,884)\end{array}$ & $\begin{array}{r}2011 \\
(n=1,804)\end{array}$ & $\begin{array}{r}2014 \\
(n=1,622)\end{array}$ & $\begin{array}{r}2011 \\
(n=1,756)\end{array}$ & $\begin{array}{r}2014 \\
(n=1,614)\end{array}$ & $\begin{array}{r}2011 \\
(n=1,861)\end{array}$ & $\begin{array}{r}2014 \\
(n=1,911)\end{array}$ & $\begin{array}{r}2011 \\
(n=1,004)\end{array}$ & $\begin{array}{r}2014 \\
(n=931)\end{array}$ & $\begin{array}{r}2011 \\
(n=8,508)\end{array}$ & $\begin{array}{r}2014 \\
(n=7,962)\end{array}$ \\
\hline The Capital and suburb & 29.4 & $34 \cdot 4$ & 29.4 & 24.9 & 28.0 & 26.4 & 31.9 & 33.4 & 36.1 & 46.7 & 30.2 & 31.3 \\
\hline Large city > 50,000 inhab. & 30.9 & 32.1 & 25.6 & 27.3 & 28.2 & 27.4 & 39.1 & 33.0 & 38.7 & 40.2 & 29.6 & 30.3 \\
\hline City $20,000-49,999$ inhab. & 31.2 & 38.1 & 29.2 & 34.9 & 28.3 & 29.8 & 37.2 & 40.6 & 28.3 & 48.1 & 29.3 & 36.5 \\
\hline Town 1,000-19,999 inhab. & 36.5 & 32.7 & 29.7 & 29.2 & 33.0 & 32.7 & 35.5 & 42.9 & 47.9 & 39.7 & 37.7 & 33.9 \\
\hline Country side & 43.1 & 31.8 & 28.4 & 31.3 & 38.0 & 31.6 & 41.8 & 38.5 & 48.9 & 57.0 & 38.5 & 37.0 \\
\hline
\end{tabular}

Note: @Weighted according to population size in the five Nordic countries.

Table 117: Proportion with high recreational screen time (> 4h/d) (\%) among adults in the Nordic countries according to 225 rbanization. NORMO 2011 and 2014

\begin{tabular}{|c|c|c|c|c|c|c|c|c|c|c|c|c|}
\hline & \multicolumn{2}{|c|}{ Denmark } & \multicolumn{2}{|c|}{ Finland } & \multicolumn{2}{|c|}{ Sweden } & \multicolumn{2}{|c|}{ Iceland } & \multicolumn{2}{|c|}{ Norway } & \multicolumn{2}{|c|}{ Nordic region@ } \\
\hline & $\begin{array}{r}2011 \\
(n=2,148)\end{array}$ & $\begin{array}{r}2014 \\
(n=1,832)\end{array}$ & $\begin{array}{r}2011 \\
(n=1,920)\end{array}$ & $\begin{array}{r}2014 \\
(n=1,565)\end{array}$ & $\begin{array}{r}2011 \\
(n=1,828)\end{array}$ & $\begin{array}{r}2014 \\
(n=1,748)\end{array}$ & $\begin{array}{r}2011 \\
(n=1,902)\end{array}$ & $\begin{array}{r}2014 \\
(n=1,884)\end{array}$ & $\begin{array}{r}2011 \\
(n=1,023)\end{array}$ & $\begin{array}{r}2014 \\
(n=994)\end{array}$ & $\begin{array}{r}2011 \\
(n=8,821)\end{array}$ & $\begin{array}{r}2014 \\
(n=8,023)\end{array}$ \\
\hline The Capital and suburb & 39.9 & 33.2 & 28.5 & 39.6 & 25.0 & 27.5 & 23.8 & 28.6 & 35.6 & 38.7 & 30.4 & 33.3 \\
\hline Large city > 50,000 inhab. & 22.6 & 40.3 & 27.5 & 36.7 & 27.3 & 27.1 & 18.6 & 23.8 & 38.3 & 26.0 & 28.3 & 31.3 \\
\hline City $20,000-49,999$ inhab. & 32.2 & 35.4 & 28.3 & 38.4 & 28.3 & 27.2 & 19.8 & 19.5 & 46.5 & 30.2 & 32.5 & 31.8 \\
\hline Town 1,000-19, 999 inhab. & 29.1 & 30.4 & 27.8 & 35.3 & 23.7 & 26.3 & 22.1 & 31.7 & 37.7 & 27.1 & 29.7 & 29.0 \\
\hline Country side & 24.8 & 22.2 & 23.4 & 25.5 & 19.6 & 18.1 & 15.7 & 14.4 & 24.7 & 34.1 & 22.3 & 23.8 \\
\hline
\end{tabular}

Note: @Weighted according to population size in the five Nordic countries.

Table 118: Prevalence of OW/OB (\%) among adults in the Nordic countries according to urbanisation. NORMO 2011 and 2014

\begin{tabular}{|c|c|c|c|c|c|c|c|c|c|c|c|c|}
\hline & \multicolumn{2}{|c|}{ Denmark } & \multicolumn{2}{|c|}{ Finland } & \multicolumn{2}{|c|}{ Sweden } & \multicolumn{2}{|c|}{ Iceland } & \multicolumn{2}{|c|}{ Norway } & \multicolumn{2}{|c|}{ Nordic region@ } \\
\hline & $\begin{array}{r}2011 \\
(n=2,148)\end{array}$ & $\begin{array}{r}2014 \\
(n=1,959)\end{array}$ & $\begin{array}{r}2011 \\
(n=1,966)\end{array}$ & $\begin{array}{r}2014 \\
(n=1,680)\end{array}$ & $\begin{array}{r}2011 \\
(n=1,820)\end{array}$ & $\begin{array}{r}2014 \\
(n=1,741)\end{array}$ & $\begin{array}{r}2011 \\
(n=1,939)\end{array}$ & $\begin{array}{r}2014 \\
(n=1,934)\end{array}$ & $\begin{array}{r}2011 \\
(n=1,026)\end{array}$ & $\begin{array}{r}2014 \\
(n=999)\end{array}$ & $\begin{array}{r}2011 \\
(n=8,899)\end{array}$ & $\begin{array}{r}2014 \\
(n=8,313)\end{array}$ \\
\hline The Capital and suburb & 32.6 & 38.7 & 41.7 & 42.0 & 39.2 & 44.2 & 51.8 & 54.7 & 35.7 & 39.3 & 38.6 & 42.1 \\
\hline Large city > 50,000 inhab. & 46.1 & 42.4 & 46.7 & 45.4 & 42.0 & 39.0 & 63.8 & 68.4 & 41.8 & 40.8 & 43.9 & 41.6 \\
\hline City $20,000-49,999$ inhab. & 45.3 & 47.2 & 46.3 & 52.2 & 46.8 & 50.4 & 68.6 & 64.8 & 51.6 & 52.2 & 47.3 & 50.4 \\
\hline Town 1,000-19,999 inhab. & 48.6 & 51.6 & 46.9 & 54.5 & 44.9 & 40.3 & 68.6 & 70.7 & 43.7 & 54.0 & 46.2 & 49.6 \\
\hline Country side & 58.6 & 58.3 & 57.6 & 50.0 & 54.2 & 52.5 & 59.4 & 67.0 & 60.1 & 51.3 & 56.9 & 52.1 \\
\hline
\end{tabular}

Note: @Weighted according to population size in the five Nordic countries. 
Table 119: Prevalence of OB (\%) among adults in the Nordic countries according to urbanisation. NORMO 2011 and 2014

\begin{tabular}{|c|c|c|c|c|c|c|c|c|c|c|c|c|}
\hline & \multicolumn{2}{|c|}{ Denmark } & \multicolumn{2}{|c|}{ Finland } & \multicolumn{2}{|c|}{ Sweden } & \multicolumn{2}{|c|}{ Iceland } & \multicolumn{2}{|c|}{ Norway } & \multicolumn{2}{|c|}{ Nordic region@ } \\
\hline & $\begin{array}{r}2011 \\
(n=2,148)\end{array}$ & $\begin{array}{r}2014 \\
(n=1,959)\end{array}$ & $\begin{array}{r}2011 \\
(n=1,966)\end{array}$ & $\begin{array}{r}2014 \\
(n=1,680)\end{array}$ & $\begin{array}{r}2011 \\
(n=1,820)\end{array}$ & $\begin{array}{r}2014 \\
(n=1,741)\end{array}$ & $\begin{array}{r}2011 \\
(n=1,939)\end{array}$ & $\begin{array}{r}2014 \\
(n=1,934)\end{array}$ & $\begin{array}{r}2011 \\
(n=1,026)\end{array}$ & $\begin{array}{r}2014 \\
(n=999)\end{array}$ & $\begin{array}{r}2011 \\
(n=8,899)\end{array}$ & $\begin{array}{r}2014 \\
(n=8,313)\end{array}$ \\
\hline The Capital and suburb & 7.9 & 10.3 & 11.7 & 14.3 & 9.5 & 5.7 & 14,5 & 17.2 & 4.0 & 10.4 & 9.1 & 10.0 \\
\hline Large city > 50,000 inhab. & 10.3 & 14.1 & 11.2 & 10.7 & 7.5 & 8.3 & 18,1 & 27.6 & 9.2 & 14.1 & 9.1 & 10.9 \\
\hline City $20,000-49,999$ inhab. & 11.6 & 15.7 & 10.0 & 16.1 & 10.7 & 11.4 & 26,1 & 27.1 & 12.5 & 16.6 & 11.2 & 14.6 \\
\hline Town 1,000-19, 999 inhab. & 15.0 & 15.1 & 11.7 & 18.5 & 9.5 & 10.4 & 29,8 & 28.5 & 6.5 & 11.4 & 10.9 & 13.4 \\
\hline Country side & 17.2 & 22.2 & 16.3 & 13.9 & 17.7 & 14.7 & 25,4 & 24.7 & 13.0 & 14.4 & 16.4 & 15.1 \\
\hline
\end{tabular}

Note: @Weighted according to population size in the five Nordic countries.

Table 120: Proportion of smokers (\%) among adults in the Nordic countries according to urbanisation. NORMO 2014

\begin{tabular}{|c|c|c|c|c|c|c|}
\hline & $\begin{array}{r}\text { Denmark } \\
2014(n=1,990)\end{array}$ & $\begin{array}{r}\text { Finland } \\
2014(n=1,696)\end{array}$ & $\begin{array}{r}\text { Sweden } \\
2014(n=1,776)\end{array}$ & $\begin{array}{r}\text { Iceland } \\
2014(n=2,001)\end{array}$ & $\begin{array}{r}\text { Norway } \\
2014(n=1,041)\end{array}$ & $\begin{array}{l}\text { Nordic region@ } \\
2014(n=8,504)\end{array}$ \\
\hline The Capital and suburb & 31.3 & 29.2 & 22.7 & 21.8 & 32.5 & 27.7 \\
\hline Large city > 50,000 inhab. & 27.7 & 19.3 & 13.4 & 24.6 & 15.7 & 17.4 \\
\hline City 20,000-49,999 inhab. & 21.4 & 22.6 & 12.9 & 20.4 & 26.7 & 19.7 \\
\hline Town 1,000-19,999 inhab. & 22.9 & 26.4 & 15.4 & 31.2 & 16.1 & 19.7 \\
\hline Country side & 29.3 & 26.3 & 13.9 & 15.0 & 20.7 & 20.0 \\
\hline
\end{tabular}

Note: @Weighted according to population size in the five Nordic countries.

Table 121: Proportion of daily smokers (\%) among adults in the Nordic countries according to urbanisation. NORMO 2014

\begin{tabular}{|c|c|c|c|c|c|c|}
\hline & Denmark & Finland & Sweden & Iceland & Norway & Nordic region@ \\
\hline & $2014(n=1,990)$ & $2014(n=1,696)$ & $2014(n=1,776)$ & $2014(n=2,001)$ & $2014(n=1,041)$ & $2014(n=8,504)$ \\
\hline The Capital and suburb & 22.7 & 17.7 & 14.3 & 13.9 & 13.9 & 17.2 \\
\hline Large city > 50,000 inhab. & 22.6 & 12.9 & 8.1 & 19.1 & 19.1 & 11.7 \\
\hline City $20,000-49,999$ inhab. & 19.5 & 18.0 & 8.9 & 14.1 & 14.1 & 14.8 \\
\hline Town 1,000-19,999 inhab. & 20.7 & 20.2 & 7.1 & 27.2 & 27.2 & 14.6 \\
\hline Country side & 24.4 & 22.4 & 9.6 & 12.3 & 12.3 & 16.0 \\
\hline
\end{tabular}

Note: @Weighted according to population size in the five Nordic countries. 
Table 122: Proportion of binge drinkers last month (\%) among adults in the Nordic countries according to urbanisation. NORMO 2014

\begin{tabular}{|c|c|c|c|c|c|c|}
\hline & $\begin{array}{r}\text { Denmark } \\
2014(n=1,991)\end{array}$ & $\begin{array}{r}\text { Finland } \\
2014(n=1,650)\end{array}$ & $\begin{array}{r}\text { Sweden } \\
2014(n=1,630)\end{array}$ & $\begin{array}{r}\text { Iceland } \\
2014(n=1,975)\end{array}$ & $\begin{array}{r}\text { Norway } \\
2014(n=1,011)\end{array}$ & $\begin{array}{l}\text { Nordic region@ } \\
2014(n=8,257)\end{array}$ \\
\hline The Capital and suburb & 47.0 & 53.5 & 42.6 & 44.5 & 56.9 & 48.6 \\
\hline Large city > 50,000 inhab. & 54.0 & 46.6 & 46.9 & 31.8 & 51.1 & 48.5 \\
\hline City $20,000-49,999$ inhab. & 45.7 & 46.8 & 43.6 & 30.2 & 53.6 & 46.6 \\
\hline Town 1,000-19,999 inhab. & 45.5 & 43.6 & 38.3 & 39.7 & 51.4 & 44.9 \\
\hline Country side & 35.9 & 32.9 & 30.7 & 21.2 & 44.8 & 34.9 \\
\hline
\end{tabular}

Note: @Weighted according to population size in the five Nordic countries.

\section{Children}

Table 123: Proportion with an unhealthy diet (\%) among children in the Nordic countries according to urbanisation. NORMO 2011 and 2014

\begin{tabular}{|c|c|c|c|c|c|c|c|c|c|c|c|c|}
\hline & \multicolumn{2}{|c|}{ Denmark } & \multicolumn{2}{|c|}{ Finland } & \multicolumn{2}{|c|}{ Sweden } & \multicolumn{2}{|c|}{ Iceland } & \multicolumn{2}{|c|}{ Norway } & \multicolumn{2}{|c|}{ Nordic region@ } \\
\hline & $\begin{array}{r}2011 \\
(n=608)\end{array}$ & $\begin{array}{r}2014 \\
(n=581)\end{array}$ & $\begin{array}{r}2011 \\
(n=496)\end{array}$ & $\begin{array}{r}2014 \\
(n=465)\end{array}$ & $\begin{array}{r}2011 \\
(n=486)\end{array}$ & $\begin{array}{r}2014 \\
(n=485)\end{array}$ & $\begin{array}{r}2011 \\
(n=490)\end{array}$ & $\begin{array}{r}2014 \\
(n=491)\end{array}$ & $\begin{array}{r}2011 \\
(n=347)\end{array}$ & $\begin{array}{r}2014 \\
(n=332)\end{array}$ & $\begin{array}{r}2011 \\
(n=2,427)\end{array}$ & $\begin{array}{r}2014 \\
(n=2,354)\end{array}$ \\
\hline The Capital and suburb & 14.3 & 20.2 & 18.2 & 16.9 & 12.1 & 24.1 & 12.1 & 11.9 & 6.3 & 7.0 & 13.3 & 19.1 \\
\hline Large city > 50,000 inhab. & 4.7 & 15.7 & 21.5 & 7.3 & 23.2 & 12.5 & 11.2 & 22.9 & 8.7 & 11.1 & 17.5 & 11.9 \\
\hline City $20,000-49,999$ inhab. & 14.1 & 10.0 & 12.3 & 14.3 & 21.3 & 19.4 & 12.4 & 15.0 & 11.5 & 23.1 & 15.0 & 16.2 \\
\hline Town 1,000-19,999 inhab. & 15.0 & 14.0 & 20.4 & 14.6 & 20.3 & 29.1 & 11.4 & 24.9 & 12.3 & 6.3 & 16.4 & 16.4 \\
\hline Country side & 5.9 & 5.9 & 17.7 & 11.4 & 19.8 & 26.5 & - & 5.1 & 8.7 & 6.8 & 14.3 & 15.6 \\
\hline
\end{tabular}

Note:

@ Weighted according to population size in the five Nordic countries. 
Table 124: Proportion of inactive (\%) among children in the Nordic countries according to urbanisation. NORMO 2011 and 2014

\begin{tabular}{|c|c|c|c|c|c|c|c|c|c|c|c|c|}
\hline & \multicolumn{2}{|c|}{ Denmark } & \multicolumn{2}{|c|}{ Finland } & \multicolumn{2}{|c|}{ Sweden } & \multicolumn{2}{|c|}{ Iceland } & \multicolumn{2}{|c|}{ Norway } & \multicolumn{2}{|c|}{ Nordic region@ } \\
\hline & $\begin{array}{r}2011 \\
(n=606)\end{array}$ & $\begin{array}{r}2014 \\
(n=586)\end{array}$ & $\begin{array}{r}2011 \\
(n=494)\end{array}$ & $\begin{array}{r}2014 \\
(n=497)\end{array}$ & $\begin{array}{r}2011 \\
(n=488)\end{array}$ & $\begin{array}{r}2014 \\
(n=495)\end{array}$ & $\begin{array}{r}2011 \\
(n=492)\end{array}$ & $\begin{array}{r}2014 \\
(n=508)\end{array}$ & $\begin{array}{r}2011 \\
(n=351)\end{array}$ & $\begin{array}{r}2014 \\
(n=351)\end{array}$ & $\begin{array}{r}2011 \\
(n=2,431)\end{array}$ & $\begin{array}{r}2014 \\
(n=2,437)\end{array}$ \\
\hline The Capital and suburb & 37.4 & 63.3 & 30.1 & 30.6 & 77.9 & 70.8 & 51.7 & 43.7 & 66.9 & 53.3 & 53.3 & 58.1 \\
\hline Large city > 50,000 inhab. & 47.7 & 59.4 & 38.7 & 44.6 & 72.3 & 61.5 & 44.2 & 54.4 & 59.6 & 65.7 & 58.6 & 58.2 \\
\hline City $20,000-49,999$ inhab. & 61.1 & 60.9 & 52.0 & 40.7 & 70.6 & 66.8 & 40.4 & 49.2 & 53.2 & 51.9 & $59 \cdot 3$ & 55.0 \\
\hline Town 1,000-19,999 inhab. & 60.9 & 54.6 & 36.9 & 41.3 & 62.6 & 78.1 & 62.6 & 51.2 & 76.7 & 63.3 & 63.5 & 62.1 \\
\hline Country side & 59.3 & 70.3 & 43.3 & 46.2 & 74.5 & 67.8 & - & 67.7 & 73.6 & 58.4 & 63.5 & 60.8 \\
\hline
\end{tabular}

Note: @Weighted according to population size in the five Nordic countries.

Table 125: Proportion with high recreational screen time (> 4h/d) (\%) among children in the Nordic countries according to urbanisation. NORMO 2011 and 2014

\begin{tabular}{|c|c|c|c|c|c|c|c|c|c|c|c|c|}
\hline & \multicolumn{2}{|c|}{ Denmark } & \multicolumn{2}{|c|}{ Finland } & \multicolumn{2}{|c|}{ Sweden } & \multicolumn{2}{|c|}{ Iceland } & \multicolumn{2}{|c|}{ Norway } & \multicolumn{2}{|c|}{ Nordic region@ } \\
\hline & $\begin{array}{r}2011 \\
(n=599)\end{array}$ & $\begin{array}{r}2014 \\
(n=578)\end{array}$ & $\begin{array}{r}2011 \\
(n=487)\end{array}$ & $\begin{array}{r}2014 \\
(n=487)\end{array}$ & $\begin{array}{r}2011 \\
(n=468)\end{array}$ & $\begin{array}{r}2014 \\
(n=489)\end{array}$ & $\begin{array}{r}2011 \\
(n=487)\end{array}$ & $\begin{array}{r}2014 \\
(n=506)\end{array}$ & $\begin{array}{r}2011 \\
(n=330)\end{array}$ & $\begin{array}{r}2014 \\
(n=334)\end{array}$ & $\begin{array}{r}2011 \\
(n=2,371)\end{array}$ & $\begin{array}{r}2014 \\
(n=2,394)\end{array}$ \\
\hline The Capital and suburb & 15.1 & 20.3 & 14.0 & 20.3 & 9.2 & 12.2 & 10.1 & 4.7 & 17.8 & 10.4 & 13.2 & 14.2 \\
\hline Large city > 50,000 inhab. & 12.9 & 24.4 & 15.9 & 18.2 & 11.0 & 14.1 & 9.9 & 5.9 & 20.4 & 12.8 & 14.3 & 16.5 \\
\hline City $20,000-49,999$ inhab. & 14.4 & 17.8 & 13.0 & 12.5 & 18.2 & 17.1 & 5.4 & 7.4 & 30.9 & 15.9 & 18.9 & 15.7 \\
\hline Town 1,000-19,999 inhab. & 18.4 & 16.0 & 21.6 & 10.7 & 16.2 & 12.3 & 3.2 & 6.2 & 21.3 & 19.4 & 18.6 & 15.1 \\
\hline Country side & 18.1 & 23.6 & 18.5 & 12.3 & 25.6 & 12.9 & - & 5.7 & 16.1 & 17.9 & 20.4 & 15.3 \\
\hline
\end{tabular}

Note: @Weighted according to population size in the five Nordic countries.

Table 126: Prevalence of OW/OB (\%) among children in the Nordic countries according to urbanisation. NORMO 2011 and 2014

\begin{tabular}{|c|c|c|c|c|c|c|c|c|c|c|c|c|}
\hline & \multicolumn{2}{|c|}{ Denmark } & \multicolumn{2}{|c|}{ Finland } & \multicolumn{2}{|c|}{ Sweden } & \multicolumn{2}{|c|}{ Iceland } & \multicolumn{2}{|c|}{ Norway } & \multicolumn{2}{|c|}{ Nordic region@ } \\
\hline & $\begin{array}{r}2011 \\
(n=559)\end{array}$ & $\begin{array}{r}2014 \\
(n=522)\end{array}$ & $\begin{array}{r}2011 \\
(n=485)\end{array}$ & $\begin{array}{r}2014 \\
(n=467)\end{array}$ & $\begin{array}{r}2011 \\
(n=444)\end{array}$ & $\begin{array}{r}2014 \\
(n=467)\end{array}$ & $\begin{array}{r}2011 \\
(n=415)\end{array}$ & $\begin{array}{r}2014 \\
(n=398)\end{array}$ & $\begin{array}{r}2011 \\
(n=289)\end{array}$ & $\begin{array}{r}2014 \\
(n=296)\end{array}$ & $\begin{array}{r}2011 \\
(n=2,192)\end{array}$ & $\begin{array}{r}2014 \\
(n=2,150)\end{array}$ \\
\hline The Capital and suburb & 3.2 & 19.4 & 10.2 & 15.8 & 18.2 & 13.9 & 14.4 & 16.4 & 25.6 & 12.4 & 13.6 & 14.9 \\
\hline Large city > 50,000 inhab. & 13.1 & 10.1 & 18.3 & 10.3 & 9.0 & 24.1 & 22.5 & 20.4 & 9.6 & 8.3 & 12.1 & 16.2 \\
\hline City 20,000-49,999 inhab. & 11.8 & 4.2 & 22.1 & 13.1 & 10.1 & 4.9 & 33.0 & 27.6 & 12.0 & 10.2 & 14.3 & 8.3 \\
\hline Town 1,000-19,999 inhab. & 13.1 & 16.2 & 19.2 & 14.6 & 22.3 & 18.7 & 29.1 & 36.6 & 15.5 & 21.5 & 17.1 & 18.0 \\
\hline Country side & 20.3 & 9.5 & 18.2 & 22.3 & 11.3 & 16.7 & - & 0.0 & 19.5 & 21.0 & 16.4 & 18.0 \\
\hline
\end{tabular}

Note: @Weighted according to population size in the five Nordic countries. 
Table 127: Prevalence of OB (\%) among children in the Nordic countries according to urbanisation. NORMO 2011 and 2014

\begin{tabular}{|c|c|c|c|c|c|c|c|c|c|c|c|c|}
\hline & \multicolumn{2}{|c|}{ Denmark } & \multicolumn{2}{|c|}{ Finland } & \multicolumn{2}{|c|}{ Sweden } & \multicolumn{2}{|c|}{ Iceland } & \multicolumn{2}{|c|}{ Norway } & \multicolumn{2}{|c|}{ Nordic region@ } \\
\hline & $\begin{array}{r}2011 \\
(n=559)\end{array}$ & $\begin{array}{r}2014 \\
(n=522)\end{array}$ & $\begin{array}{r}2011 \\
(n=485)\end{array}$ & $\begin{array}{r}2014 \\
(n=467)\end{array}$ & $\begin{array}{r}2011 \\
(n=444)\end{array}$ & $\begin{array}{r}2014 \\
(n=467)\end{array}$ & $\begin{array}{r}2011 \\
(n=415)\end{array}$ & $\begin{array}{r}2014 \\
(n=398)\end{array}$ & $\begin{array}{r}2011 \\
(n=289)\end{array}$ & $\begin{array}{r}2014 \\
(n=296)\end{array}$ & $\begin{array}{r}2011 \\
(n=2,192)\end{array}$ & $\begin{array}{r}2014 \\
(n=2,150)\end{array}$ \\
\hline The Capital and suburb & 0.0 & 11.6 & 3.6 & 0.0 & 3.4 & 1.3 & 1.6 & 3.8 & 13.0 & 5.0 & 4.1 & 2.9 \\
\hline Large city > 50,000 inhab. & 0.0 & 1.2 & 2.9 & 1.0 & 0.8 & 6.4 & 7.9 & 1.3 & 2.0 & 1.4 & 1.6 & 3.5 \\
\hline City 20,000-49,999 inhab. & 1.7 & 1.3 & 4.1 & 0.4 & 0.0 & 1.5 & 4.2 & 3.1 & 7.9 & 0.0 & 3.4 & 0.9 \\
\hline Town 1,000-19, 999 inhab. & 1.4 & 6.1 & 4.1 & 9.7 & 2.8 & 2.4 & 12.3 & 6.6 & 1.0 & 6.0 & 2.0 & 5.4 \\
\hline Country side & 7.7 & 3.3 & 4.3 & 2.7 & 2.2 & 3.1 & - & 0.0 & 5.1 & 1.7 & 4.2 & 2.7 \\
\hline
\end{tabular}

Note: @Weighted according to population size in the five Nordic countries. 
Nordic Council of Ministers

Ved Stranden 18

DK-1061 Copenhagen K

www.norden.org

\section{The Nordic Monitoring System 2011-2014}

This report describes the results of the first and second collection of data in the joint Nordic Monitoring System of diet, physical activity and overweight. It is well recognized that health in the European Region needs to be improved and Nordic public health researchers have since 2008 developed and validated a Nordic method to assess diet and physical activity among adults and children. Data has collected on health behaviour and overweight in 2011 and 2014 among 18,000 adults and 5,000 children. During the three year survey period, significant changes have occurred in the Nordic countries, especially among adults. The Nordic Monitoring System shows that lifestyle and weight status differ between the Nordic countries despite highly comparable societal structures. Favorable and unfavorable health behaviour is present in all countries and there are areas to be improved in all countries.

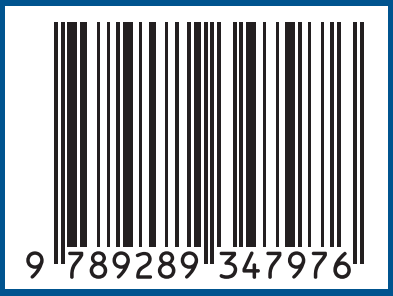

